Supporting Information for

\title{
Switchable Catalysts Used to Control Suzuki Cross-Coupling and Aza-Michael Addition/Asymmetric Transfer Hydrogenation Cascade Reactions
}

\author{
Jingjing Meng, Fengwei Chang, Yanchao Su, Rui Liu, Tanyu Cheng, and Guohua Liu * \\ Key Laboratory of Resource Chemistry of Ministry of Education, Shanghai Key Laboratory of Rare \\ Earth Functional Materials, Shanghai Normal University, Shanghai 200234, PR China.
}

\section{CONTENTS}

Experimental.

Figure S1. FT-IR spectra of material 1 and catalysts $\mathbf{2 , 5}$ and $\mathbf{6}$

Figure S2. XPS spectra of the homogeneous $\mathrm{Pd}_{2}(\mathrm{dba})_{3}$, the homogeneous mixed $\operatorname{Pd}_{2}(\mathrm{dba})_{3}$ and $\mathrm{Pd}\left(\mathrm{Me}_{2} \mathrm{PCH}_{2}=\mathrm{CHCON}^{i} \mathrm{Pr}\right)_{2}$ and catalyst 5

Figure S3. TG/DTA curves of 1-4

Figure S4. Solid-state ${ }^{13} \mathrm{C} \mathrm{CP/MAS} \mathrm{NMR} \mathrm{spectra} \mathrm{of} \mathrm{catalysts} 5$ and $\mathbf{6}$ . .55

Figure S5. Solid-state ${ }^{29}$ Si MAS NMR spectra of catalysts $\mathbf{2}, \mathbf{5}$ and $\mathbf{6}$

Figure S6. Nitrogen adsorption-desorption isotherms of catalysts $\mathbf{2 , 5}$ and $\mathbf{6}$.

Figure S7. (a) The TEM image with a chemical mapping of $\mathbf{5}$ showing the distribution of Si (white), Pd (green) and Ru (red). (b) The SEM images of the reused catalyst 5 at the seventh run

Figure S8. (a) SEM images of catalyst 6, (b) TEM images of catalyst 6, and the dispersive situations of catalyst 5 in water as indicated $35^{\circ} \mathrm{C}$ (c) and $15{ }^{\circ} \mathrm{C}(\mathrm{d})$, and a TEM image with a chemical mapping of 6 showing the distribution of $\mathrm{Si}$ (white) and $\mathrm{Ru}$ (red).

Figure S9. Average hydrodynamic diameters distribution measurement for the recycled catalyst $\mathbf{5}$ as indicated at $15^{\circ} \mathrm{C}$ and at $35^{\circ} \mathrm{C}$..

Figure S10. Average hydrodynamic diameters distribution measurement for the recycled catalyst $\mathbf{6}$ as indicated at $15^{\circ} \mathrm{C}$ and at $35^{\circ} \mathrm{C}$..

Table S1. Investigation of reaction switching in the cascade reaction of 1-phenylprop-2-enone and aniline

Figure S11. Time course in the 6-catalyzed transformation of 1-phenylprop-2-enone (10a) and aniline (11a)

Figure S12. HPLC analyses for chiral products..

Table S2. Reusability of catalysts 5 for the Suzuki cross-coupling/ATH of cascade reactions of 4-chloroacetophenone and phenylboronic acid.

Figure S13. Reusability of catalyst 5 for the Suzuki cross-coupling/ATH of cascade reactions of 4-chloroacetophenone and phenylboronic acid..

Table S3. Reusability of catalyst 6 for the aza-Michal addition/ATH cascade reaction of 1-phenylprop2-enone and aniline.

Figure S14. Reusability of catalyst 6 for the aza-Michal addition/ATH cascade reaction of 1phenylprop-2-enone and aniline.

Figure S15. Characterizations of chiral products.. 


\section{Experimental}

1. General: All experiments, which are sensitive to moisture or air, were carried out under an Ar atmosphere using the standard Schlenk techniques. Tetraethoxysilane (TEOS), 1,4-bis(triethyoxysilyl)ethane, cetyltrimethylammonium bromide (CTAB), fluorocarbon surfactant

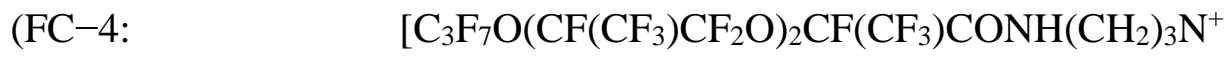

$\left.\left.\left(\mathrm{C}_{2} \mathrm{H}_{5}\right)_{2} \mathrm{CH}_{3}\right] \mathrm{I}^{-}\right)$, 4-(2-(trimethoxysilyl)ethyl)benzene-1-sulfonyl chloride, 4-(methylphenylsulfonyl)-1,2-diphenylethylenediamine, $\quad N$-Isopropylacrylamide, $\quad \operatorname{Pd}_{2}\left(\mathrm{dba}_{3}\right)_{3}$, trimethylphosphine, acrylamide, acrylonitrile, 2,2-azobisisobutyronitrile, (Mesitylene $\left.\mathrm{RuCl}_{2}\right)_{2}$ were purchased from Sigma-Aldrich Company Ltd and used as received. The compound of $(S, S)-4-$ (trimethoxysilyl)ethyl)phenylsulfonyl-1,2-diphenylethylenediamine was synthesized according to the reported literature [(a) J. Mater. Chem., 2010, 20, 1970].

2. Characterization: $\mathrm{Ru}$ and $\mathrm{Pd}$ loading amounts in the catalysts were analyzed using an inductively coupled plasma optical emission spectrometer (ICP, Varian VISTA-MPX). Fourier transform infrared (FT-IR) spectra were collected on a Nicolet Magna 550 spectrometer using $\mathrm{KBr}$ method. Scanning electron microscopy (SEM) images were obtained using a JEOL JSM-6380LV microscope operating at $20 \mathrm{kV}$. Transmission electron microscopy (TEM) images were performed on a JEOL JEM2010 electron microscope at an acceleration voltage of $220 \mathrm{kV}$. Nitrogen adsorption isotherms were measured at $77 \mathrm{~K}$ with a Quantachrome Nova 4000 analyzer. The samples were measured after being outgassed at $423 \mathrm{~K}$ overnight. Pore size distributions were calculated by using the BJH model. The specific surface areas $\left(S_{\mathrm{BET}}\right)$ of samples were determined from the linear parts of BET plots $\left(p / p_{0}=0.05-1.00\right)$. Solid-state NMR experiments were explored on a Bruker AVANCE spectrometer at a magnetic field strength of 9.4 T with ${ }^{1} \mathrm{H}$ frequency of $400.1 \mathrm{MHz},{ }^{13} \mathrm{C}$ frequency of $100.5 \mathrm{MHz}$ and ${ }^{29} \mathrm{Si}$ frequency of $79.4 \mathrm{MHz}$ with 4 $\mathrm{mm}$ rotor at two spinning frequency of $5.5 \mathrm{kHz}$ and $8.0 \mathrm{kHz}$, TPPM decoupling is applied in the during acquisition period. ${ }^{1} \mathrm{H}$ cross polarization in all solid-state NMR experiments was employed using a contact time of $2 \mathrm{~ms}$ and the pulse lengths of $4 \mu \mathrm{s}$.

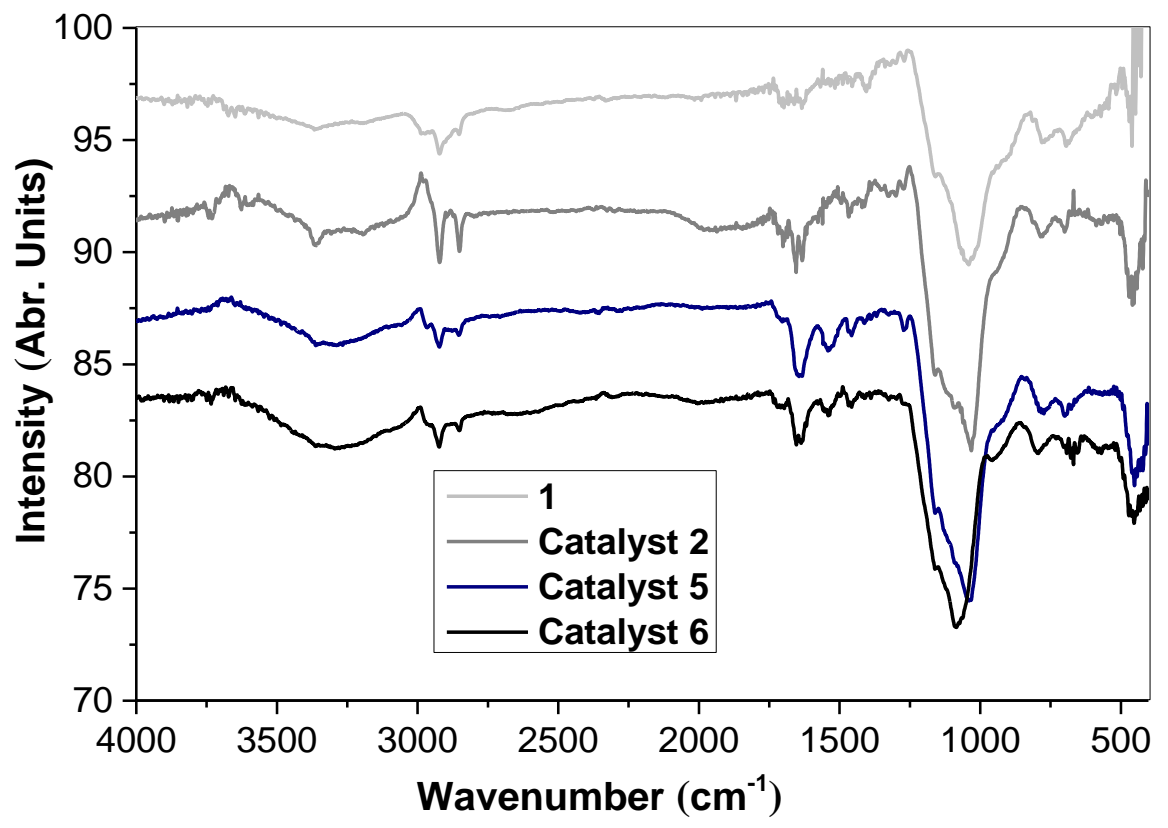

Figure S1. FT-IR spectrum of material 1 and catalysts 2, 5 and 6. 


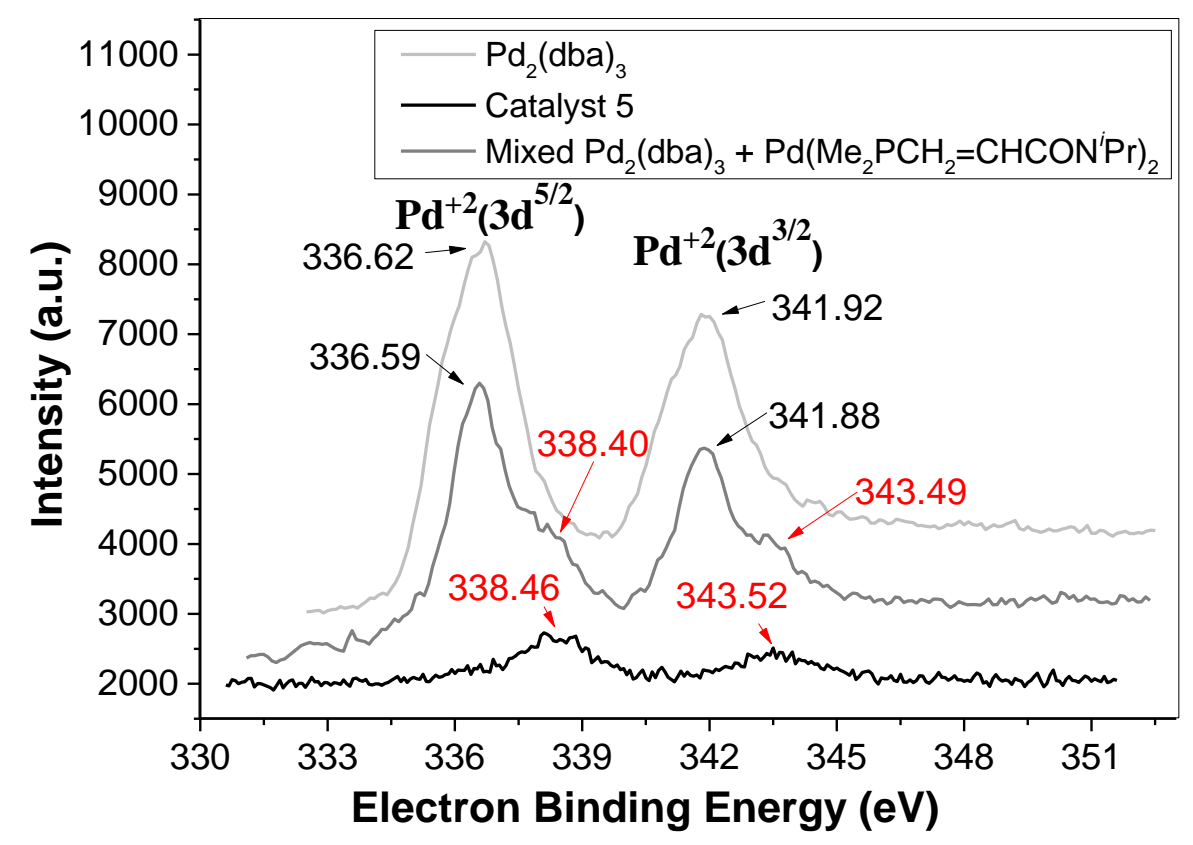

Figure S2. XPS spectra of the homogeneous $\mathrm{Pd}_{2}(\mathrm{dba})_{3}$, the homogeneous mixed $\mathrm{Pd}_{2}(\mathrm{dba})_{3}$ and $\mathrm{Pd}\left(\mathrm{Me}_{2} \mathrm{PCH}_{2}=\mathrm{CHCON}^{i} \mathrm{Pr}\right)_{2}$ and catalyst 5
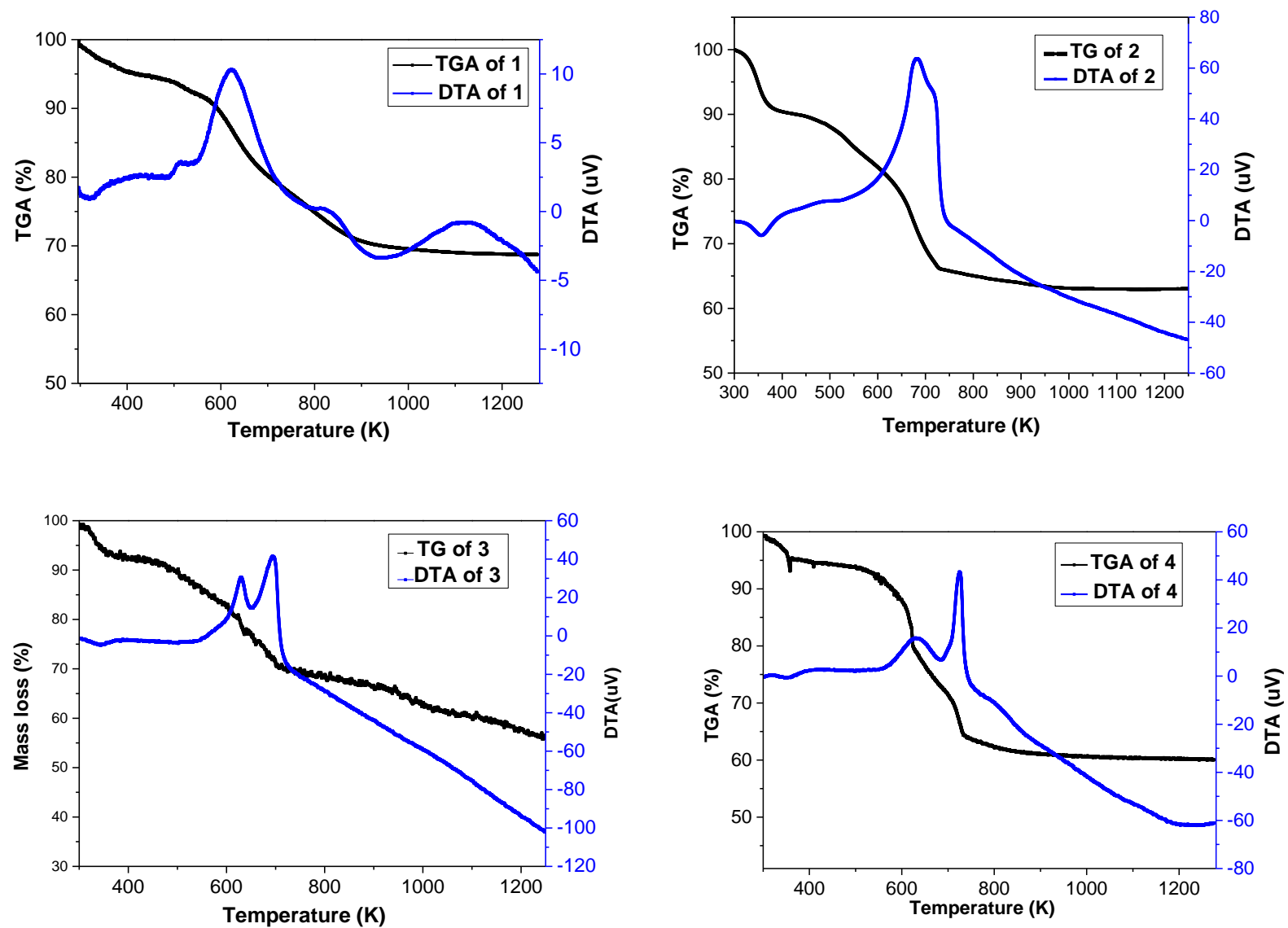

Figure S3. TG/DTA curves of 1-4. 
Explanation: The TG/DTA curves of the Vinyl@ArDPEN@HS (1) was treated in the air as shown above. An endothermic peak around $322 \mathrm{~K}$ with weight loss of (100\%-94.7\%) 5.3\% could be attributed to the release of physical adsorption water while the comprehensive endothermic peaks between $440 \mathrm{~K}$ and $1200 \mathrm{~K}$ with weight loss of (94.7\%-69.0\%) $25.7 \%$ could be assigned to the oxidation of organic molecules (including alkyl-linked vinyl groups or ArDPEN moiety, alkyl fragments and part of the residual surfactant). Apparently, total weight loss of the oxidation of organic molecules was $25.7 \%$ per $94.7 \%$ the extracted samples when eliminated the part of water, meaning the $27.14 \%$ weight loss of the oxidation of organic molecules per $100 \%$ materials.

The TG/DTA curves of the catalyst 2 was treated in the air as shown above. An endothermic peak around $356 \mathrm{~K}$ with weight loss of (100\%-89.6\%) $10.1 \%$ could be attributed to the release of physical adsorption water while the comprehensive endothermic peaks between $440 \mathrm{~K}$ and $1200 \mathrm{~K}$ with weight loss of (89.6\%-62.9\%) $26.7 \%$ could be assigned to the oxidation of organic molecules (including alkyllinked vinyl groups or alkyl-linked MesityleneRuArDPEN complexes, alkyl fragments and part of the residual surfactant). Apparently, total weight loss of the oxidation of organic molecules was $26.7 \%$ per $89.6 \%$ the extracted samples when eliminated the part of water, meaning the $29.79 \%$ weight loss of the oxidation of organic molecules per $100 \%$ materials.

As compared the weight loss of 2 with 1, the weight loss of the MesityleneCl moieties was 2.65\% (29.79-27.14) per $100 \%$ materials. Also, this finding means that the mole amounts of [MesityleneCl] in 2 is $0.01704 \mathrm{~mol} \%(\mathrm{Mr}=155.5)$, demonstrating the mole amount of chiral ligands in catalyst $\mathbf{2}$ is $0.01704 \mathrm{~mol} \%$. This value means the contents of chiral ligands $\left(0.01704\right.$ mol\%*380.1 $\left.\left(\operatorname{Mr}_{(\operatorname{ArDPEN})}\right)\right)$ in catalyst $\mathbf{2}$ is $6.48 \%$. It was worth mentioning that the $0.01704 \mathrm{~mol} \%$ of [MesityleneCl] in 2 means 17.31 $\mathrm{mg}(0.1704 \mathrm{mmol}$ of $\mathrm{Ru})$ of the $\mathrm{Ru}$ loading per gram of $\mathbf{2}$, which is also consistent with the value (the $\mathrm{Ru}$ loading was $17.12 \mathrm{mg}(0.168 \mathrm{mmol})$ per gram of catalyst $)$ detected by ICP-OES analysis.

For the polymer-coating $\mathrm{P}_{1} @$ ArDPEN@HS (3), it was found easily that a similar endothermic peak around $346 \mathrm{~K}$ with weight loss of (100\%-92.1\%) 7.9\% was strongly similar to that of parent $\mathbf{1}$ due to the release of physical adsorption water. It was worth mentioning that the all exothermic peaks were combined into one complicated exothermic peak between $440 \mathrm{~K}$ and $1200 \mathrm{~K}$ with weight loss of (92.1\%-57.5\%) 34.6\% could be assigned to the oxidation of organic molecules(including alkyl-linked polymer, alkyl-linked ArDPEN moiety, alkyl fragments and part of the residual surfactant). Because of the total weight loss of organic moieties was $34.6 \%$ per $92.1 \%$ the extracted catalyst when eliminated the part of water, meaning the whole weight loss $37.56 \%$ of the oxidation of the organic molecules per $100 \%$ materials.

As compared the weight loss of $\mathbf{3}$ with $\mathbf{1}$, the weight loss of the outer thermoresponsive polymercoating layers was $10.42 \%(37.56 \%-27.14 \%)$ per $100 \%$ materials.

For the polymer-coating $\mathrm{P}_{2} @$ ArDPEN@HS (4), it was found easily that a similar endothermic peak around $353 \mathrm{~K}$ with weight loss of (100\%-94.5\%) 5.5\% was strongly similar to that of 1 due to the release of physical adsorption water. It was worth mentioning that the all exothermic peaks were combined into one complicated exothermic peak between $440 \mathrm{~K}$ and $1200 \mathrm{~K}$ with weight loss of (94.5\%-61.0\%) 33.5\% could be assigned to the oxidation of organic molecules (including alkyl-linked polymer, alkyl-linked ArDPEN moiety, alkyl fragments and part of the residual surfactant). Because the total weight loss of organic moieties was $33.5 \%$ per $94.5 \%$ the extracted catalyst when eliminated the part of water, meaning the whole weight loss $35.45 \%$ of the oxidation of the organic molecules per $100 \%$ materials.

As compared the weight loss of $\mathbf{4}$ with $\mathbf{1}$, the weight loss of the outer thermoresponsive polymercoating layers was $8.31 \%(35.45 \%-27.14 \%)$ per $100 \%$ materials. 

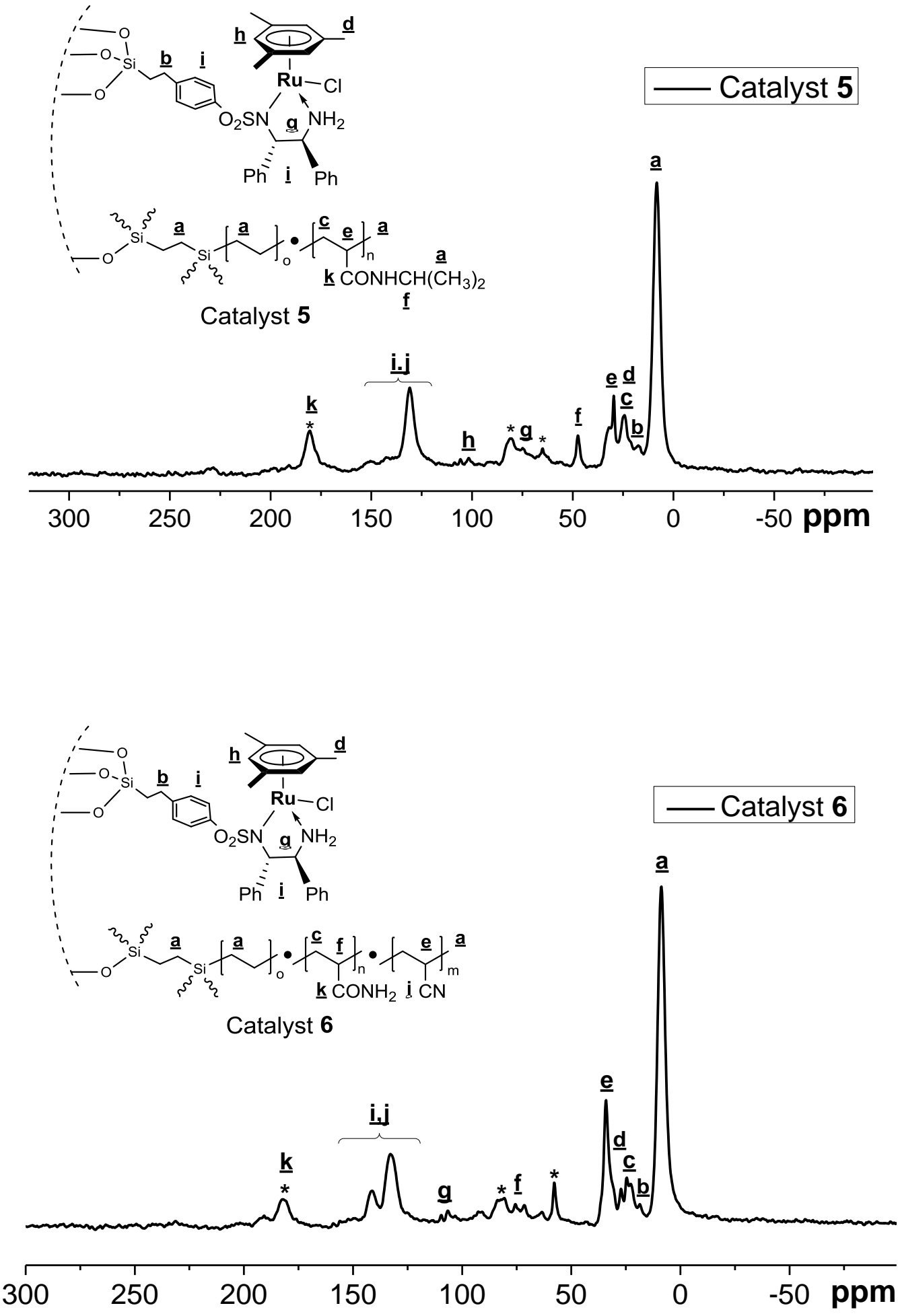

Figure S4. Solid-state ${ }^{13} \mathrm{C}$ CP/MAS NMR spectra of catalysts 5 and $\mathbf{6}$. 


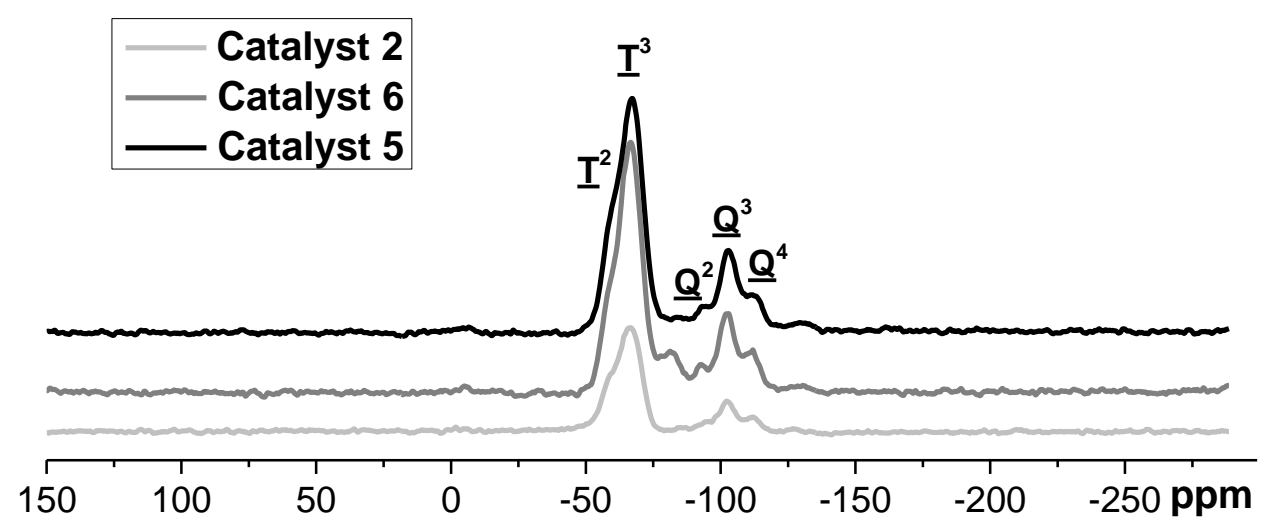

Figure S5. Solid- state $^{29}$ Si MAS NMR spectra of catalysts 2, 5 and $\mathbf{6}$.

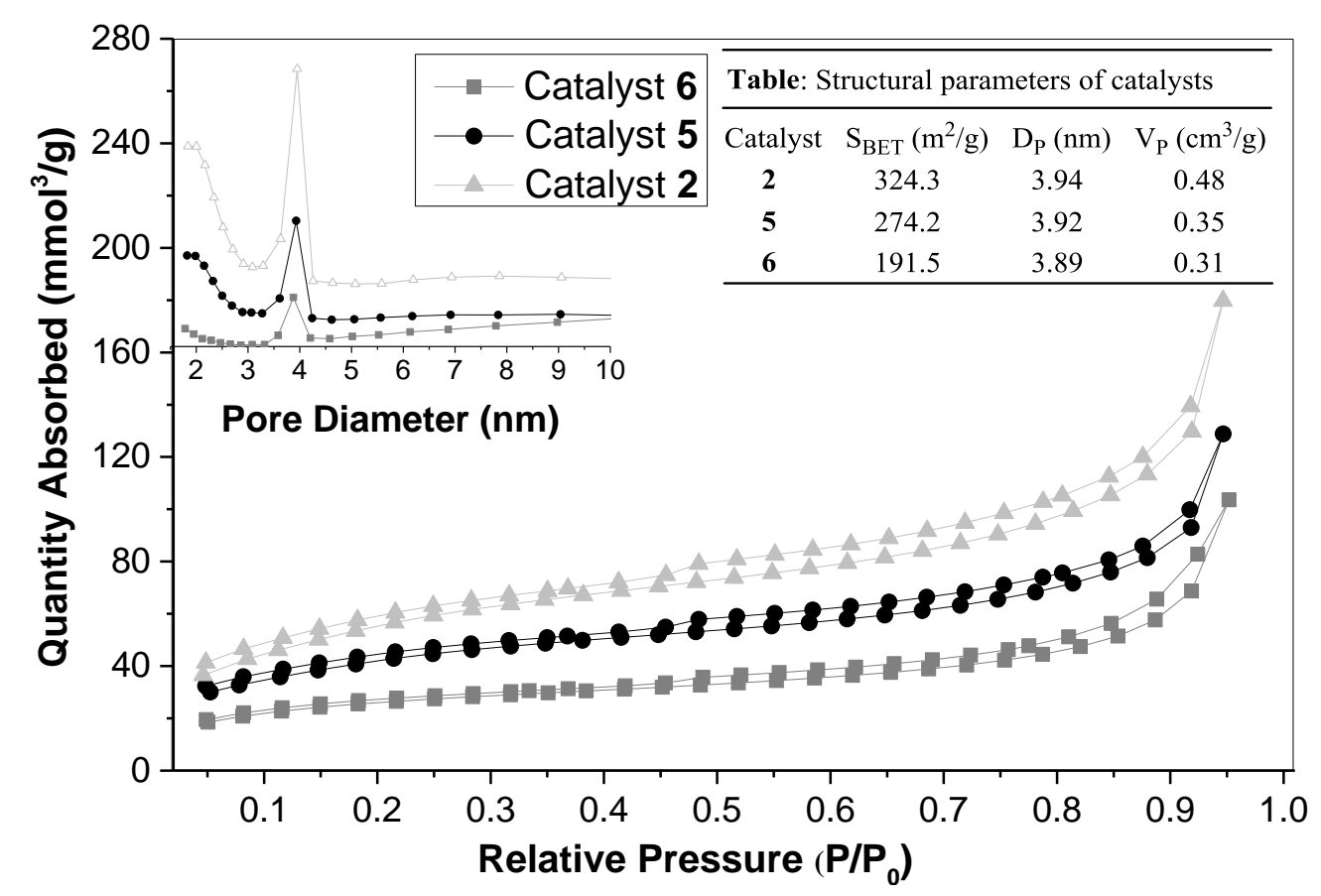

Figure S6. Nitrogen adsorption-desorption isotherms of catalysts 2, 5 and $\mathbf{6}$. 

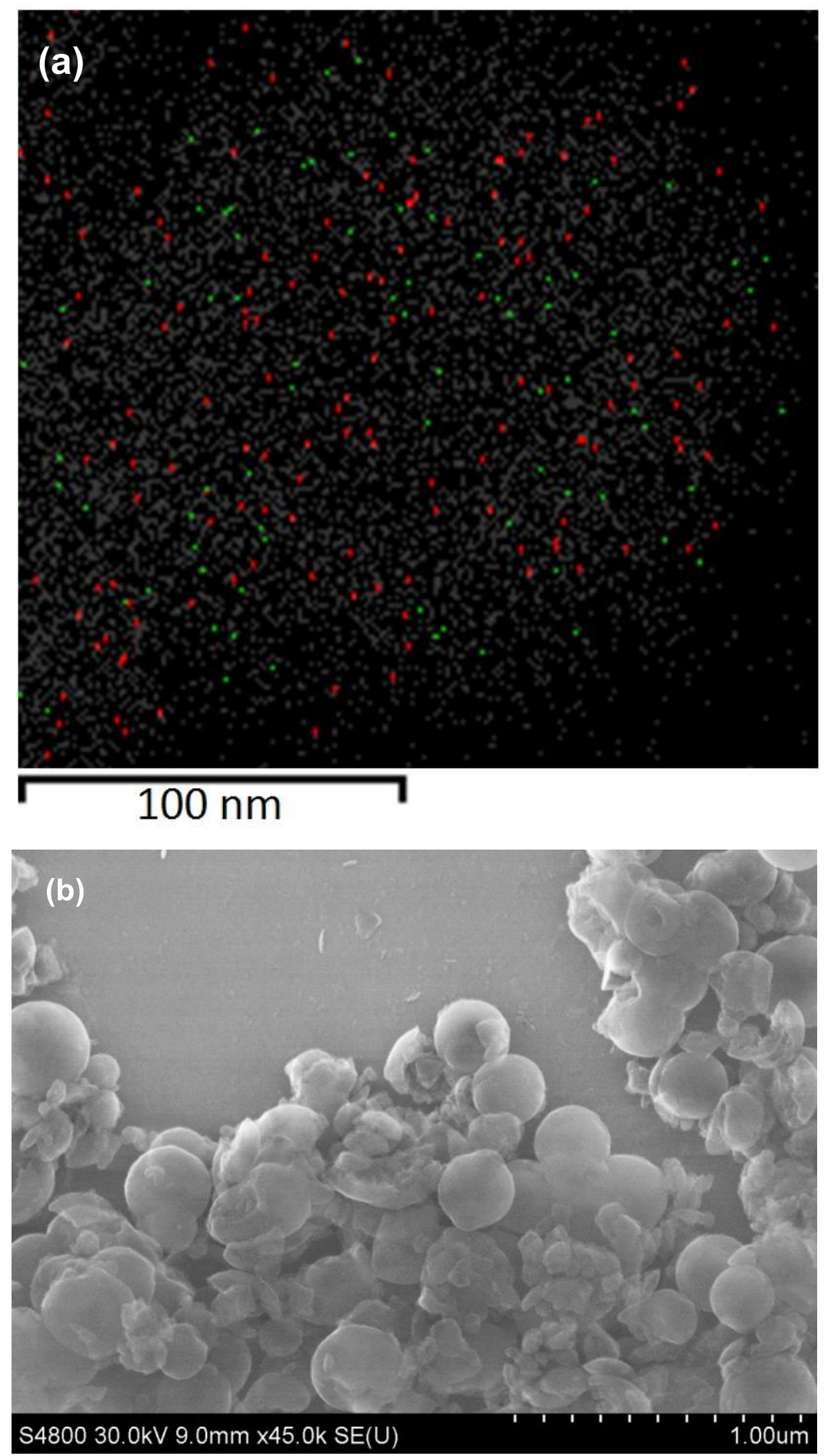

Figure S7. (a) The TEM image with a chemical mapping of 5 showing the distribution of Si (gray), Pd (green) and Ru (red). (b) The SEM images of the reused catalyst 5 at the seventh run. 

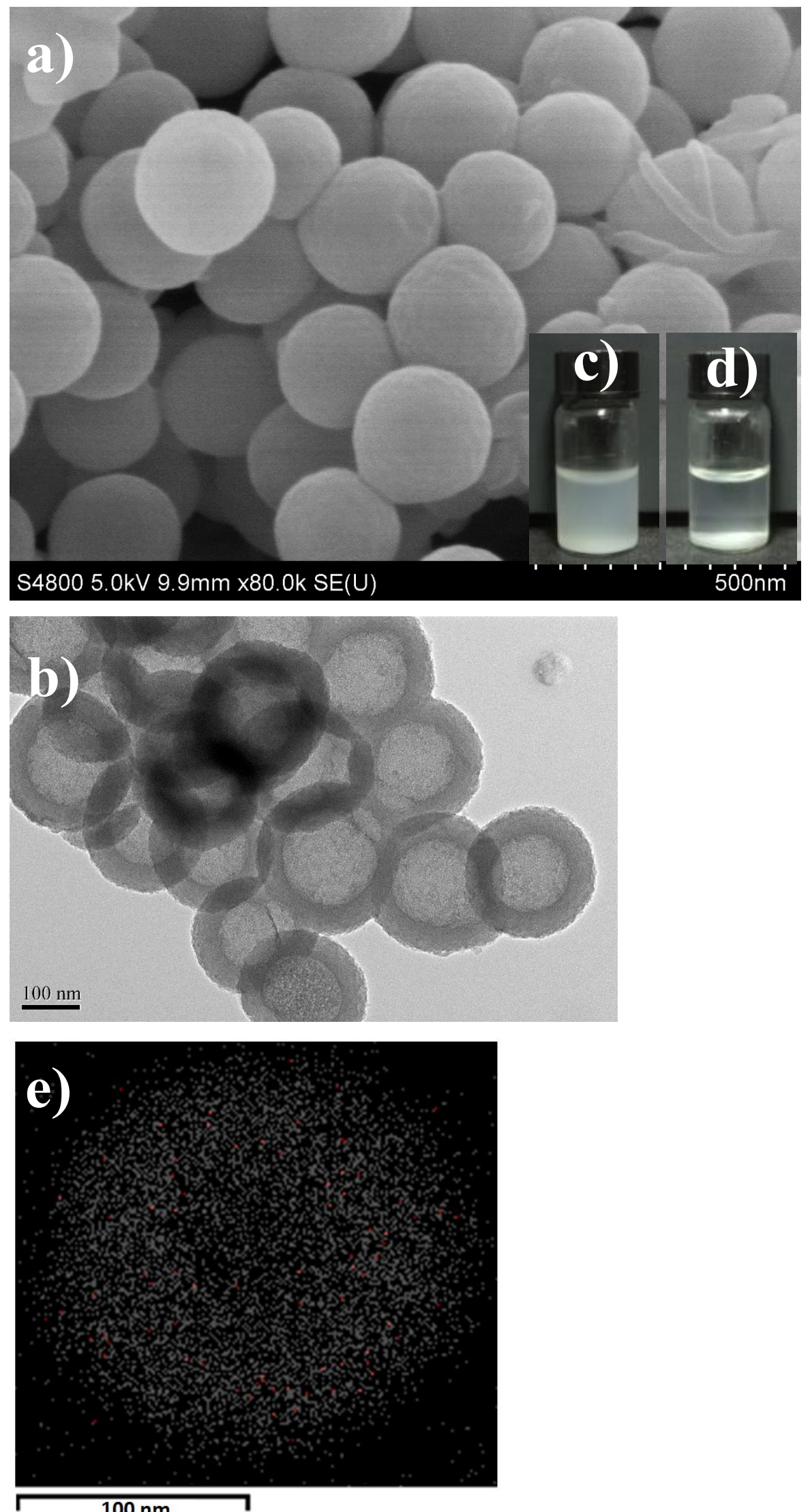

Figure S8. (a) SEM images of catalyst 6, (b) TEM images of catalyst 6 , the dispersive situations of catalyst 6 in water as indicated $35^{\circ} \mathrm{C}(\mathrm{c})$ and $15^{\circ} \mathrm{C}(\mathrm{d})$, and (e) a TEM image with a chemical mapping of 6 showing the distribution of $\mathrm{Si}$ (white) and $\mathrm{Ru}$ (red). 
Results $\left(15^{\circ} \mathrm{C}\right.$ for first run)

$\begin{array}{rlllll} & & & \text { Size (d.n... } & \text { \% Number } & \text { Width (d.n... } \\ \text { Z-Average (d.nm): } & 491.6 & \text { Peak 1: } & 402.9 & 99.9 & 160.7 \\ \text { Pdl: } & 0.205 & \text { Peak 2: } & 5040 & 0.1 & 789.9 \\ \text { Intercept: } & 0.909 & \text { Peak 3: } & 0.000 & 0.0 & 0.000 \\ \text { Result quality } & \text { Good } & & & & \end{array}$

Size Distribution by Number

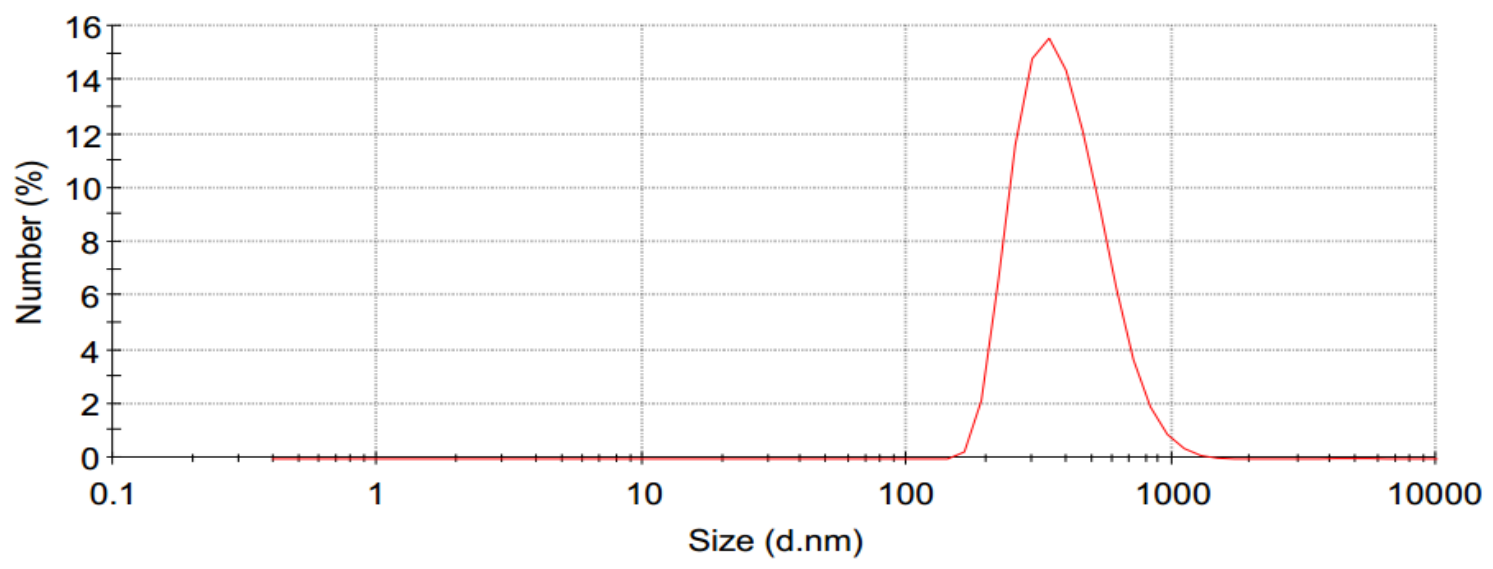

Results $\left(35^{\circ} \mathrm{C}\right.$ for first run)

Z-Average (d.nm): 392.8

Peak 1: $\quad 255.8$

$\%$ Number

Width (d.n...

Pdl: 0.375

Peak 2: 2054

99.2

67.88

Intercept: 0.922

Peak 3: $\quad 0.000$

0.8

801.0

Result quality Good

$\begin{array}{ll}0.0 & 0.000\end{array}$

Size Distribution by Number

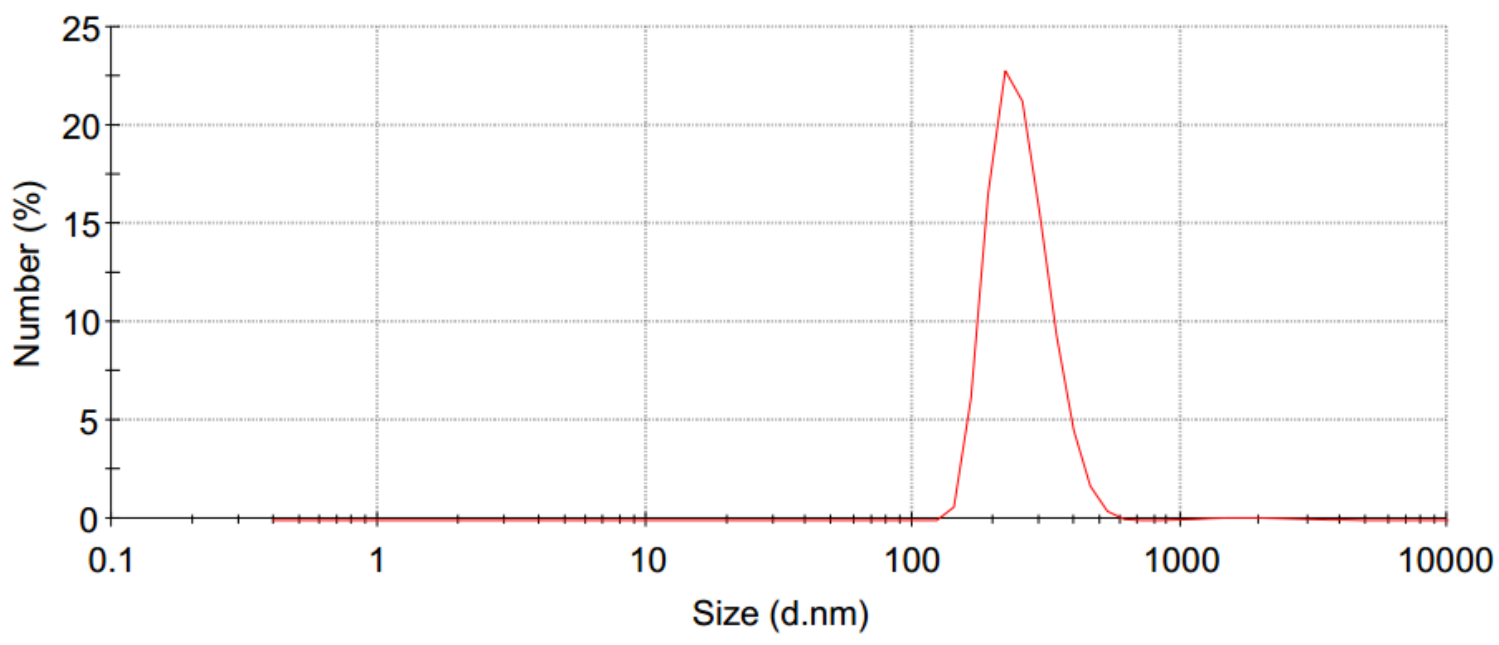


Results $\left(15^{\circ} \mathrm{C}\right.$ for second run)

$\begin{array}{rlllll} & & & \text { Size (d.n... } & \% \text { Number } & \text { Width (d.n... } \\ \text { Z-Average (d.nm): } & 482.2 & \text { Peak 1: } & 153.6 & 30.3 & 26.23 \\ \text { Pdl: } & 0.165 & \text { Peak 2: } & 388.1 & 69.7 & 183.3 \\ \text { Intercept: } & 0.899 & \text { Peak 3: } & 0.000 & 0.0 & 0.000 \\ \text { Result quality } & \text { Good } & & & & \end{array}$

Size Distribution by Number

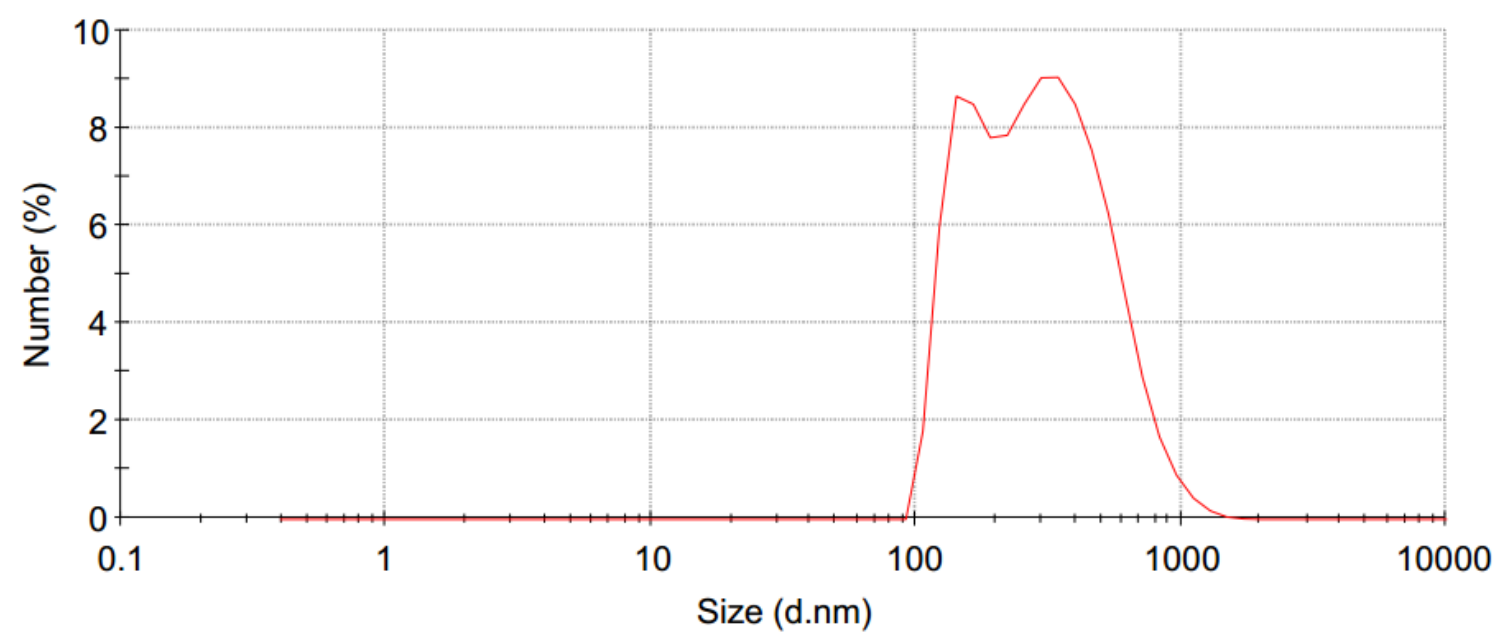

Results (35 ${ }^{\circ} \mathrm{C}$ for second run)

$\begin{array}{rlllll} & & & \text { Size (d.n... } & \text { \% Number } & \text { Width (d.n... } \\ \text { Z-Average (d.nm): } & 405.9 & \text { Peak 1: } & 302.4 & 99.9 & 107.3 \\ \text { Pdl: } & 0.289 & \text { Peak 2: } & 5214 & 0.1 & 723.4 \\ \text { Intercept: } & 0.869 & \text { Peak 3: } & 0.000 & 0.0 & 0.000 \\ \text { Result quality } & \text { Good } & & & & \end{array}$

Size Distribution by Number

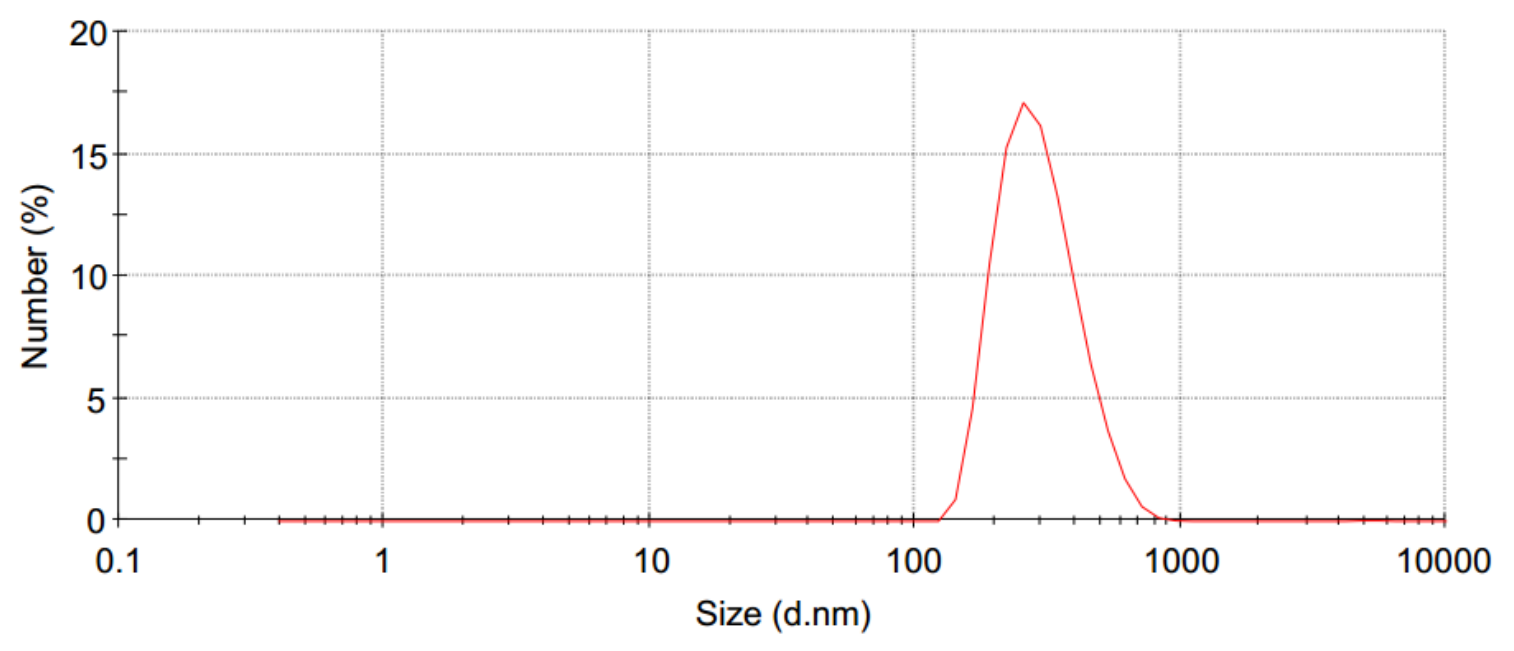


Results ( $15^{\circ} \mathrm{C}$ for third run)

Z-Average (d.nm): 492.3

Pdl: 0.287

Intercept: 0.890

Size (d.n... \% Number Width (d.n...

Peak 1: $\quad 349.4$

99.3

117.0

Peak 2: 2988

0.7

1129

Peak 3: $\quad 0.000$

0.0

0.000

\section{Result quality Good}

Size Distribution by Number

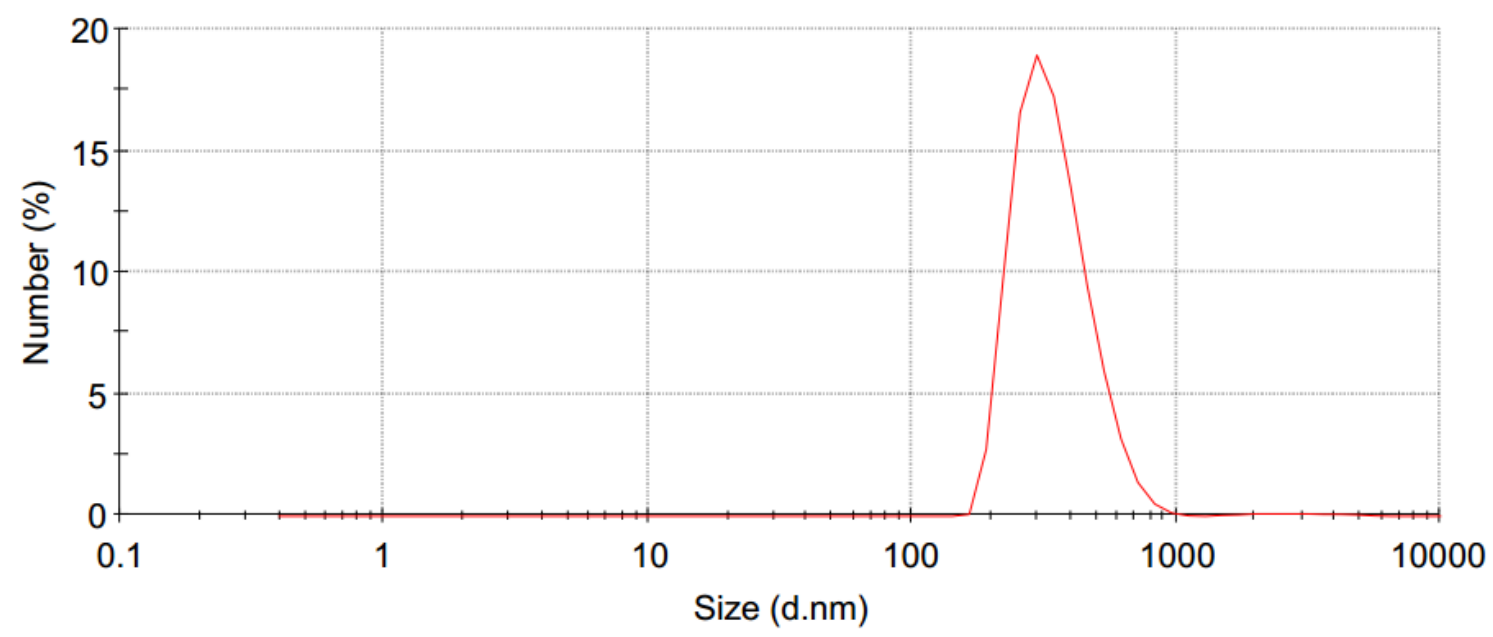

Results ( $35^{\circ} \mathrm{C}$ for third run)

Z-Average (d.nm): 399.2

Pdl: 0.272

Intercept: 0.914

Result quality Good

Size (d.n... \% Number Width (d.n...

Peak 1: $\quad 325.9$

100.0

74.74

Peak 2: $\quad 0.000$

0.0

0.000

Peak 3: $\quad 0.000$

0.0

0.000

Size Distribution by Number

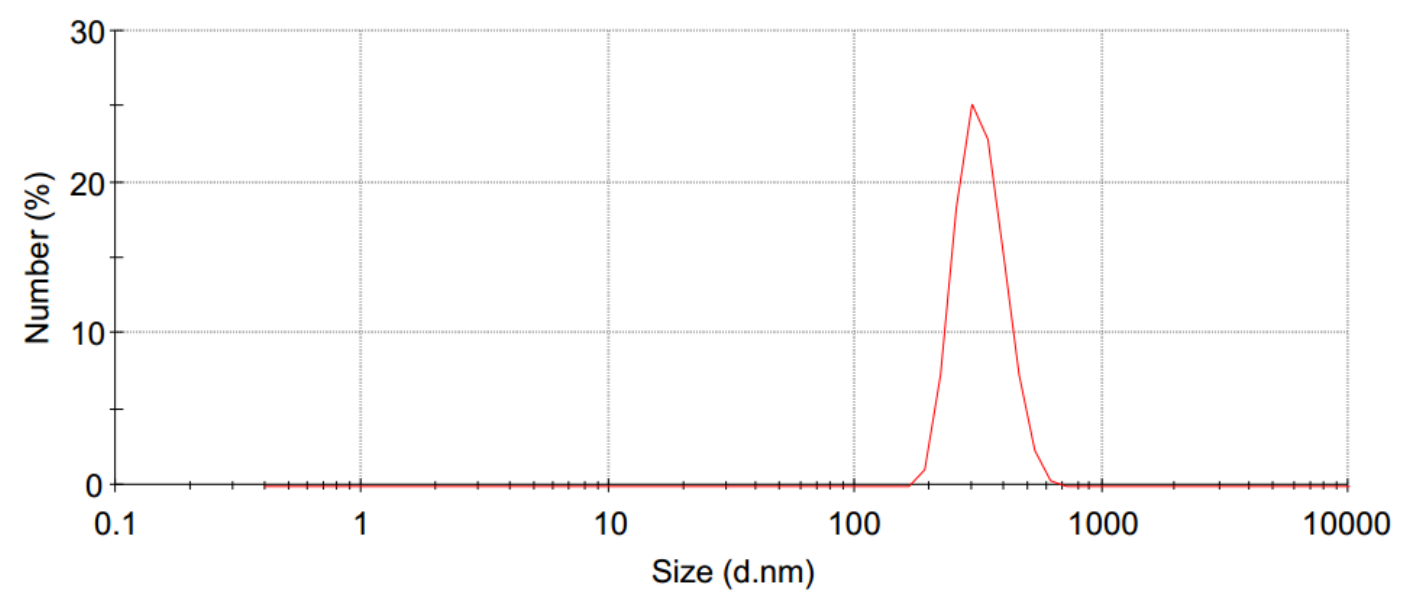


Results $\left(15^{\circ} \mathrm{C}\right.$ for fourth run)

Z-Average (d.nm): 480.8

Pdl: 0.159

Intercept: 0.905

Result quality Good

Size (d.n... \% Number Width (d.n...

$\begin{array}{llll}\text { Peak 1: } & 448.3 & 100.0 & 141.9\end{array}$

$\begin{array}{llll}\text { Peak 2: } & 0.000 & 0.0 & 0.000\end{array}$

$\begin{array}{llll}\text { Peak 3: } & 0.000 & 0.0 & 0.000\end{array}$

Size Distribution by Number

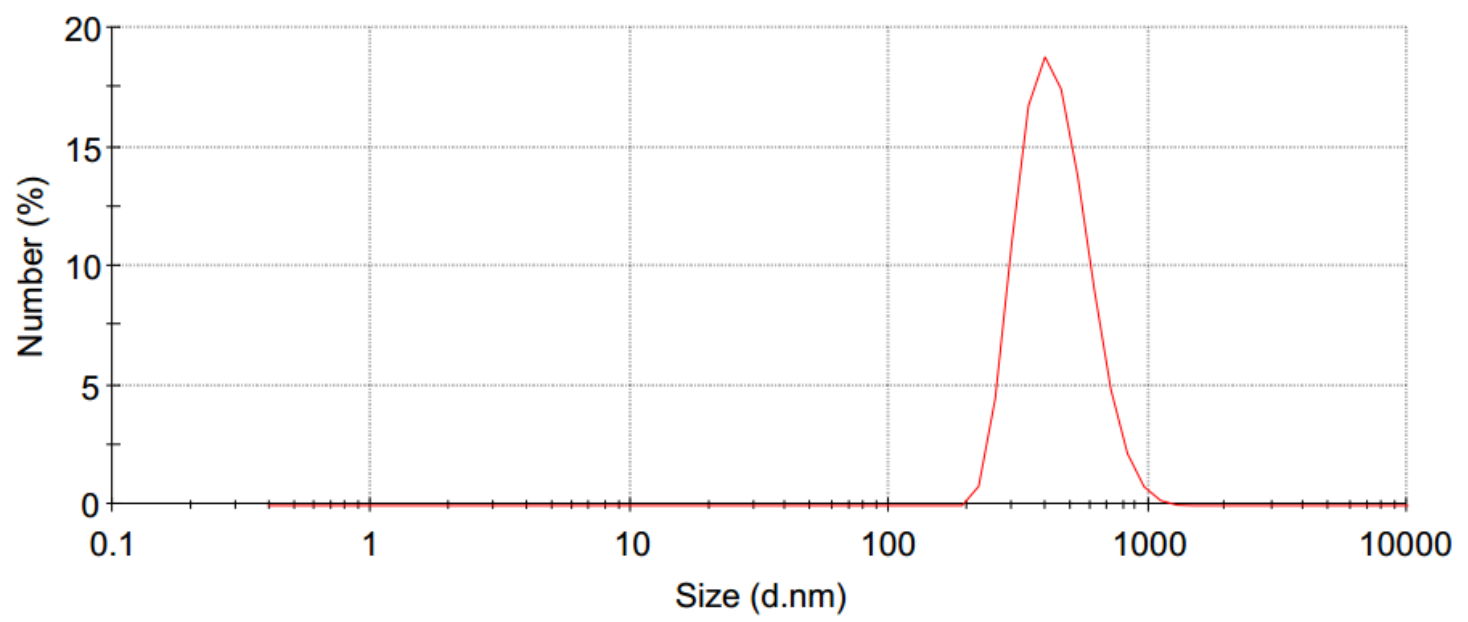

Results (35 ${ }^{\circ} \mathrm{C}$ for fourth run)

Z-Average (d.nm): 407.5

Pdl: 0.254

Peak 1: $\quad 355.6$

100.0

Width (d.n...

Peak 2: $\quad 0.000$

0.0

84.61

Intercept: 0.905

Peak 3: $\quad 0.000$

0.0

0.000

Result quality Good

Size Distribution by Number

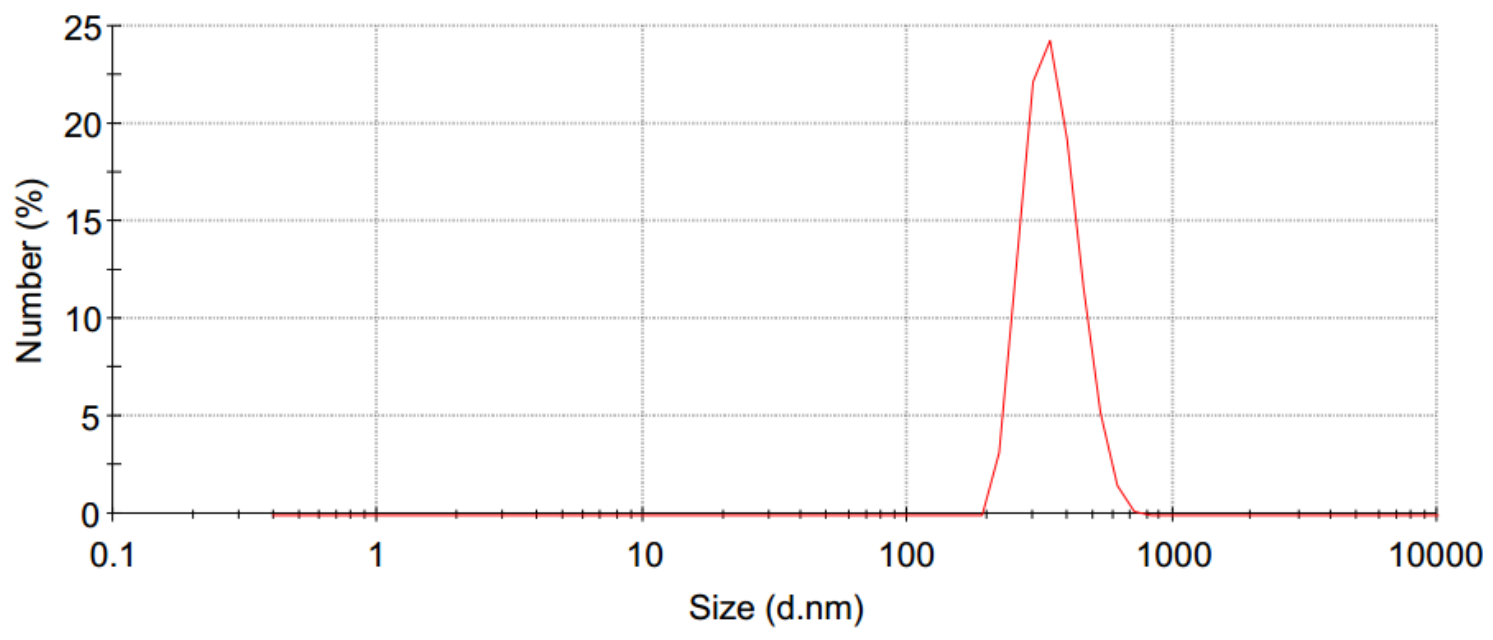


Results $\left(15^{\circ} \mathrm{C}\right.$ for fifth run)

$\begin{array}{rlllll} & & & \text { Size (d.n... } & \text { \% Number } & \text { Width (d.n... } \\ \text { Z-Average (d.nm): } & 490.4 & \text { Peak 1: } & 352.6 & 99.8 & 143.9 \\ \text { Pdl: } & 0.296 & \text { Peak 2: } & 4322 & 0.2 & 1049 \\ \text { Intercept: } & 0.879 & \text { Peak 3: } & 0.000 & 0.0 & 0.000 \\ \text { Result quality } & \text { Good } & & & & \end{array}$

Size Distribution by Number

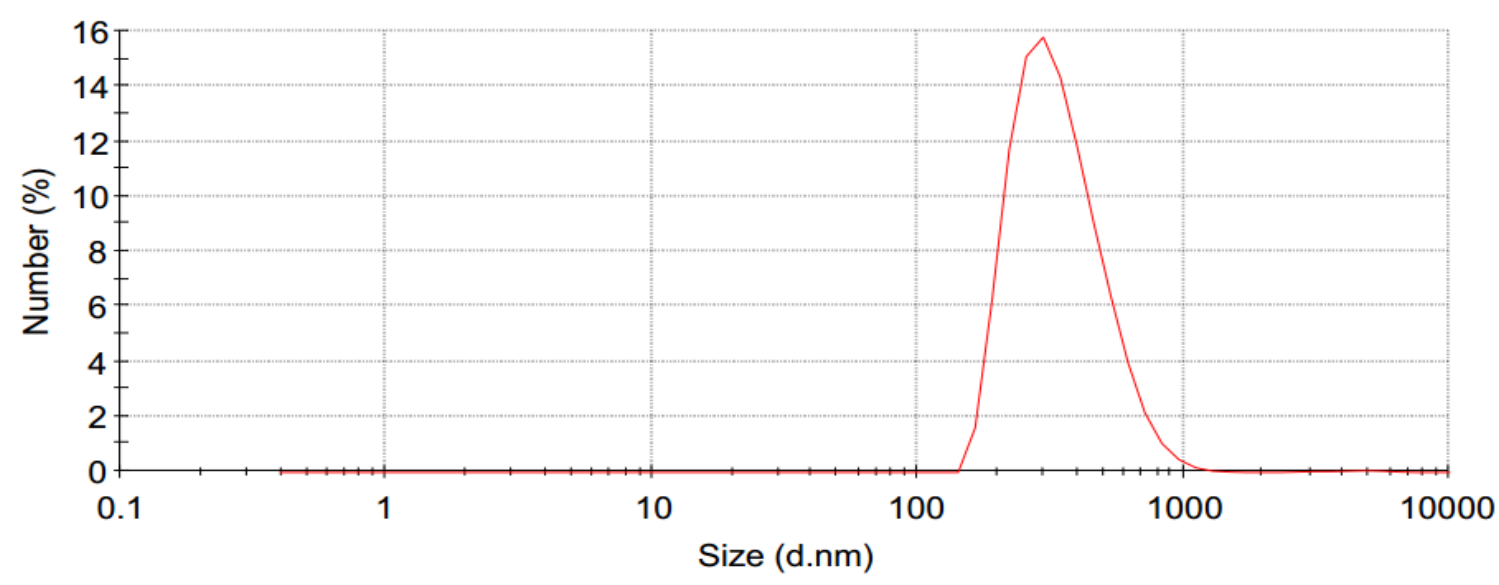

\section{Results ( $35^{\circ} \mathrm{C}$ for fifth run)}

Z-Average (d.nm): 402.7

Pdl: 0.255

Intercept: 0.912

Result quality Good

Size (d.n... \% Number Width (d.n...

$\begin{array}{llll}\text { Peak 1: } & 349.8 & 100.0 & 82.35\end{array}$

$\begin{array}{llll}\text { Peak 2: } & 0.000 & 0.0 & 0.000\end{array}$

$\begin{array}{llll}\text { Peak 3: } & 0.000 & 0.0 & 0.000\end{array}$

Size Distribution by Number

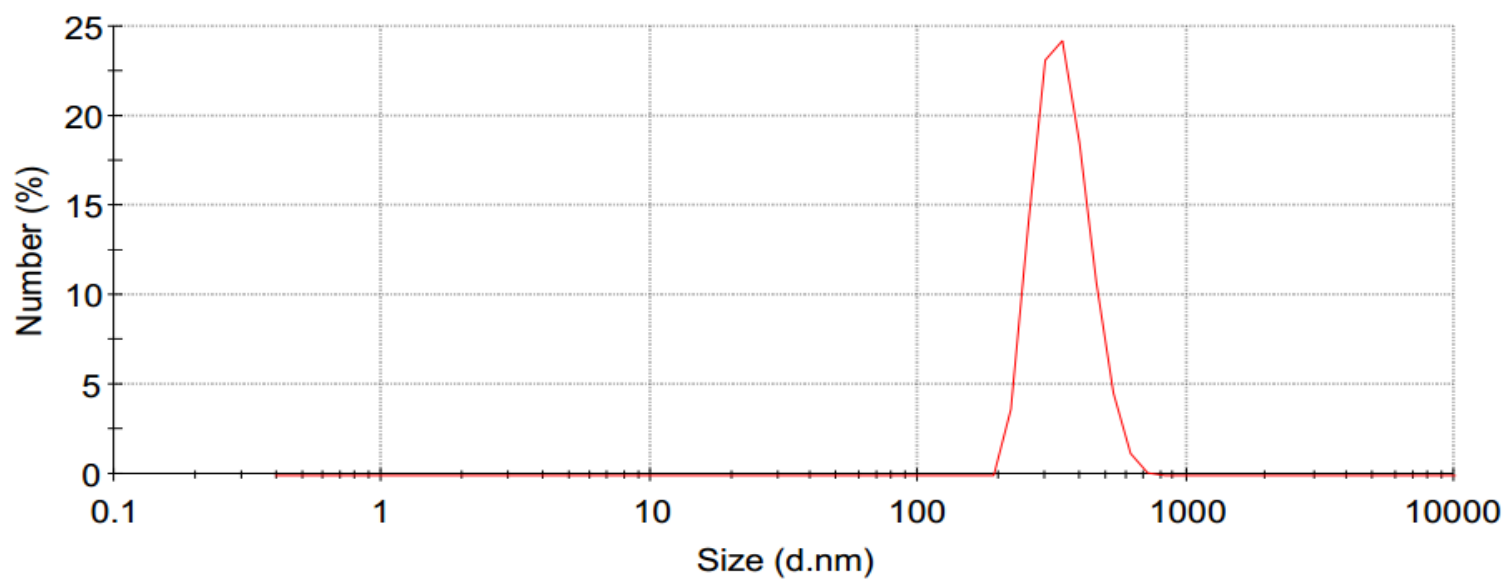

Figure S9. Average hydrodynamic diameters distribution measurement for the recycled catalyst $\mathbf{5}$ as indicated at $15^{\circ} \mathrm{C}$ and at $35^{\circ} \mathrm{C}$. 
Results ( $15^{\circ} \mathrm{C}$ for first run)

Z-Average (d.nm): 421.0

Size (d.n... \% Number Width (d.n...

$$
\begin{aligned}
\text { Pdl: } & 0.205 \\
\text { Intercept: } & 0.907 \\
\text { Result quality } & \text { Good }
\end{aligned}
$$

Peak 1: $\quad 401.1$

18.2

166.6

Peak 2: 122.6

81.8

30.26

Peak 3: $\quad 0.000$

0.0

0.000

Size Distribution by Number

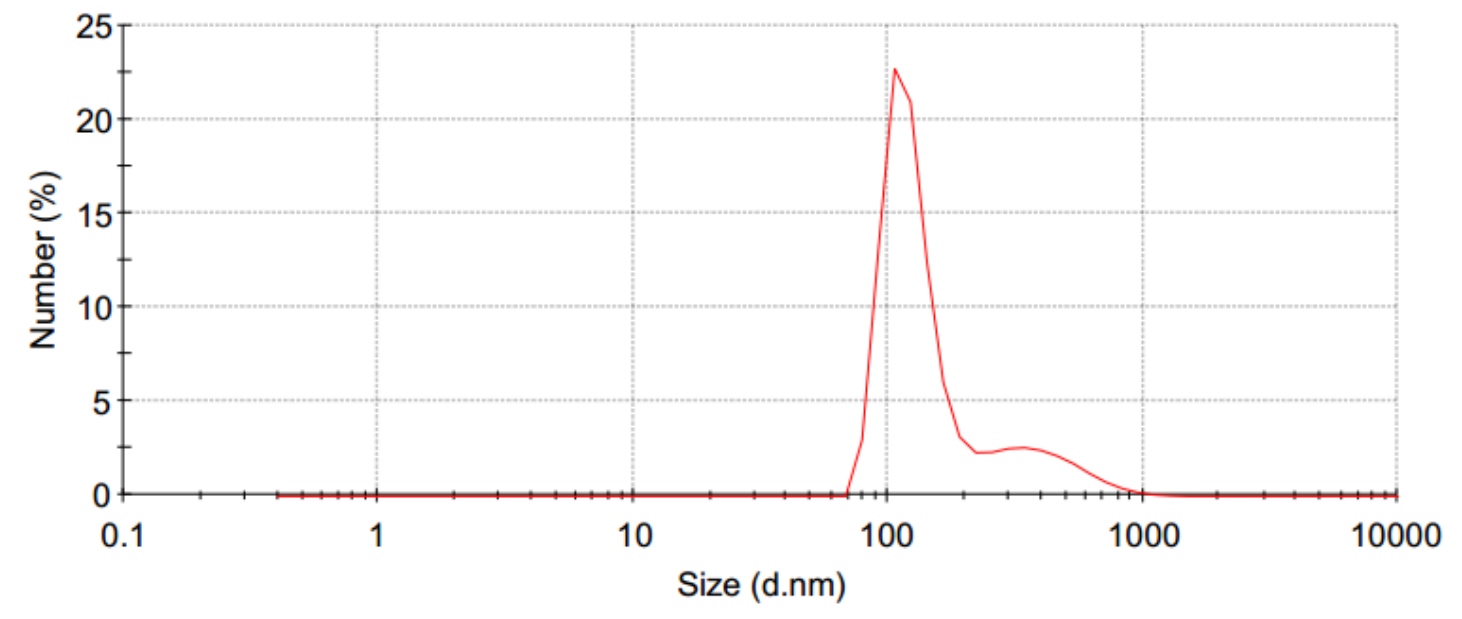

Results (35 ${ }^{\circ} \mathrm{C}$ for first run)

Size (d.n... \% Number Width (d.n...

Z-Average (d.nm): 492.5

Peak 1: $\quad 333.6$

98.9

92.62

Pdl: 0.353

Peak 2: 2595

1.1

1002

Intercept: 0.957

Peak 3: $\quad 0.000$

0.0

0.000

\section{Result quality Good}

Size Distribution by Number

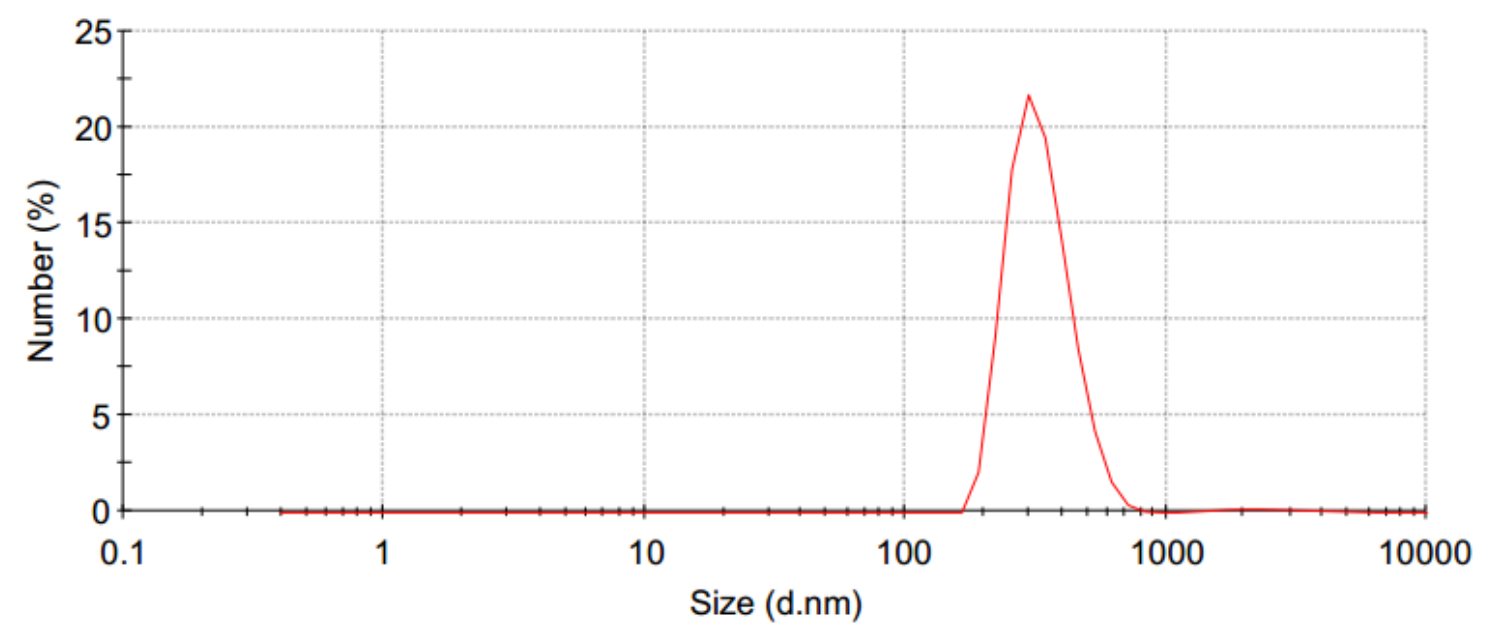


Results ( $15^{\circ} \mathrm{C}$ for second run)

Size (d.n... \% Number Width (d.n...

Z-Average (d.nm): 416.4

Pdl: 0.250

Intercept: 0.915

Result quality Good

$\begin{array}{llll} & \text { Size (d.n... } & \% \text { Number } & \text { Width (d.n... } \\ \text { Peak 1: } & 210.7 & 100.0 & 145.2 \\ \text { Peak 2: } & 4773 & 0.0 & 886.9 \\ \text { Peak 3: } & 0.000 & 0.0 & 0.000\end{array}$

Size Distribution by Number

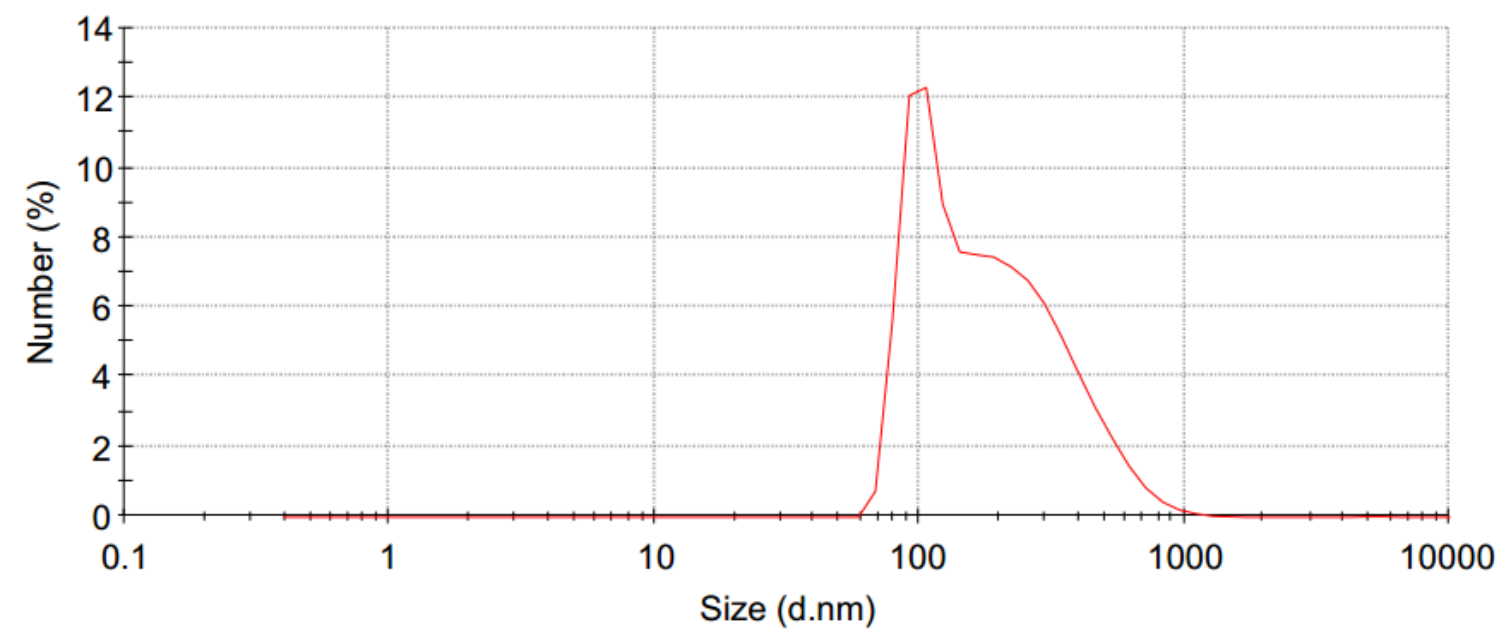

Results ( $35^{\circ} \mathrm{C}$ for second run)

Z-Average (d.nm): 502.0

$\begin{aligned} \text { Pdl: } & 0.340 \\ \text { Intercept: } & 0.943 \\ \text { Result quality } & \text { Good }\end{aligned}$

$\begin{aligned} \text { Pdl: } & 0.340 \\ \text { Intercept: } & 0.943 \\ \text { Result quality } & \text { Good }\end{aligned}$

Size (d.n... \% Number Width (d.n...

Peak 1: 298.2

98.0

82.14

Peak 2: 1775

2.0

636.0

Peak 3: $\quad 0.000$

0.0

0.000

Size Distribution by Number

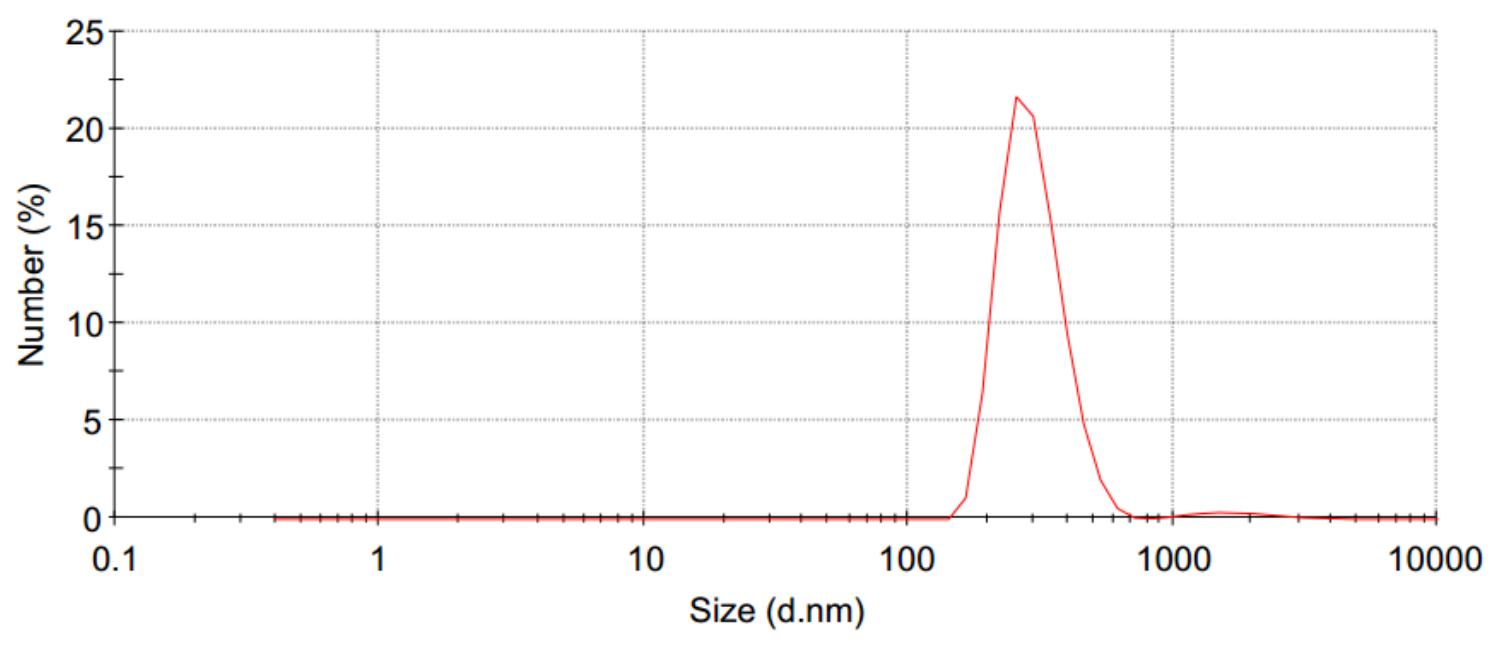


Results $\left(15^{\circ} \mathrm{C}\right.$ for third run)

Z-Average (d.nm): 417.7

$\begin{aligned} \text { Pdl: } & 0.241 \\ \text { Intercept: } & 0.906 \\ \text { Result quality } & \text { Good }\end{aligned}$

$\begin{aligned} \text { Pdl: } & 0.241 \\ \text { Intercept: } & 0.906 \\ \text { Result quality } & \text { Good }\end{aligned}$

Size (d.n... \% Number Width (d.n...

Peak 1: $\quad 347.2$

100.0

104.0

Peak 2: $\quad 5265$

0.0

705.8

Peak 3: $\quad 0.000$

0.0

0.000

Size Distribution by Number

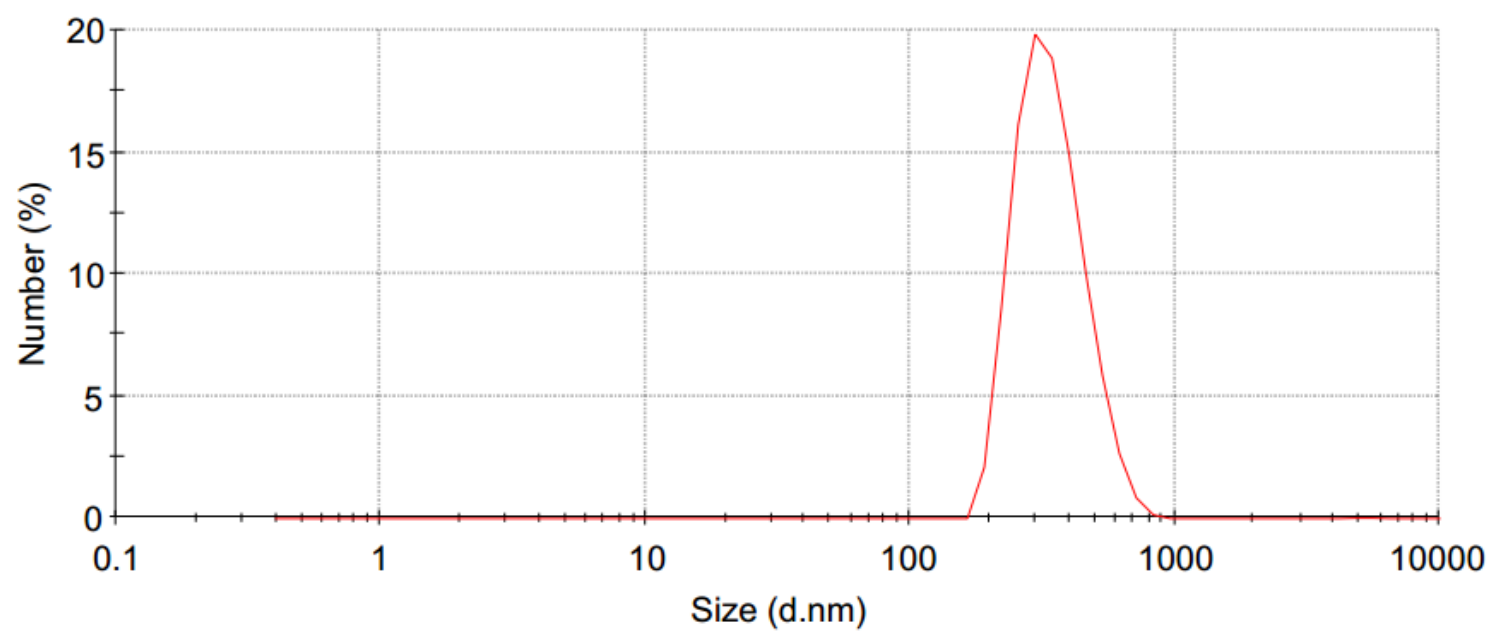

Results $\left(35^{\circ} \mathrm{C}\right.$ for third run)

Z-Average (d.nm): 470.2

Pdl: 0.426

Intercept: 0.944

Result quality Good

Size (d.n... \% Number Width (d.n...

Peak 1: $\quad 303.8$

99.0

78.39

Peak 2: 2706

1.0

999.4

Peak 3: $\quad 0.000$

0.0

0.000

Size Distribution by Number

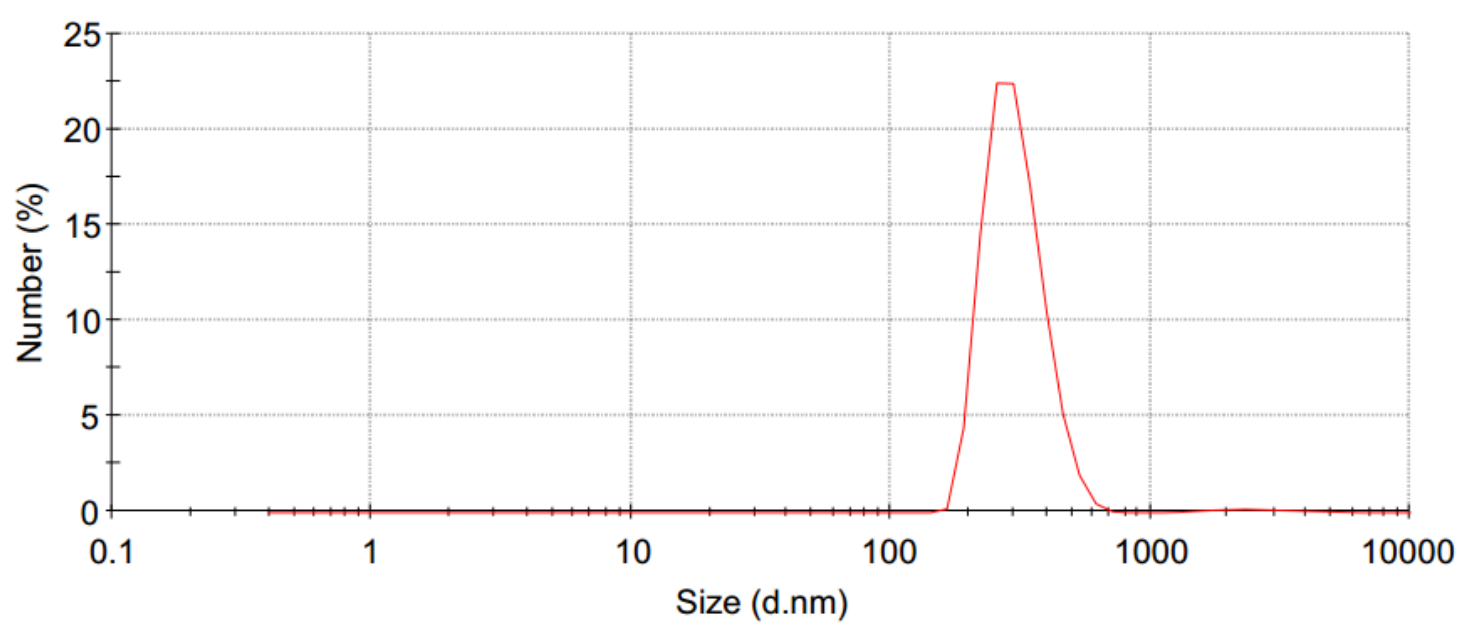


Results ( $15^{\circ} \mathrm{C}$ for fourth run)

Z-Average (d.nm): 405.4

Pdl: 0.230

Peak 1: $\quad 348.7$

100.0

Width (d.n...

Intercept: 0.905

Peak 2: 5408

0.0

94.61

Peak 3: $\quad 0.000$

0.0

649.3

Result quality Good

Size Distribution by Number

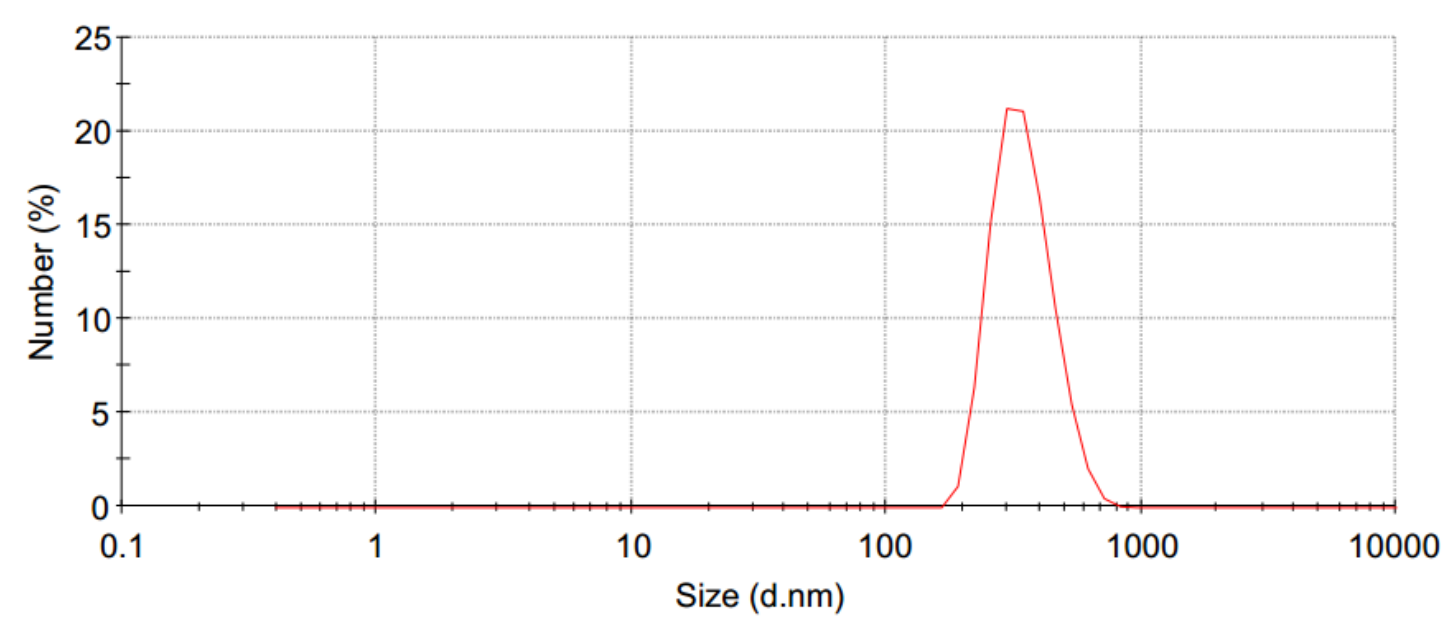

Results ( $35^{\circ} \mathrm{C}$ for fourth run)

$\begin{array}{rlllll} & & & \text { Size (d.n... } & \% \text { Number } & \text { Width (d.n... } \\ \text { Z-Average (d.nm): } & 483.1 & \text { Peak 1: } & 365.7 & 100.0 & 75.40 \\ \text { Pdl: } & 0.365 & \text { Peak 2: } & 0.000 & 0.0 & 0.000 \\ \text { Intercept: } & 0.928 & \text { Peak 3: } & 0.000 & 0.0 & 0.000 \\ \text { Result quality } & \text { Good } & & & & \end{array}$

Size Distribution by Number

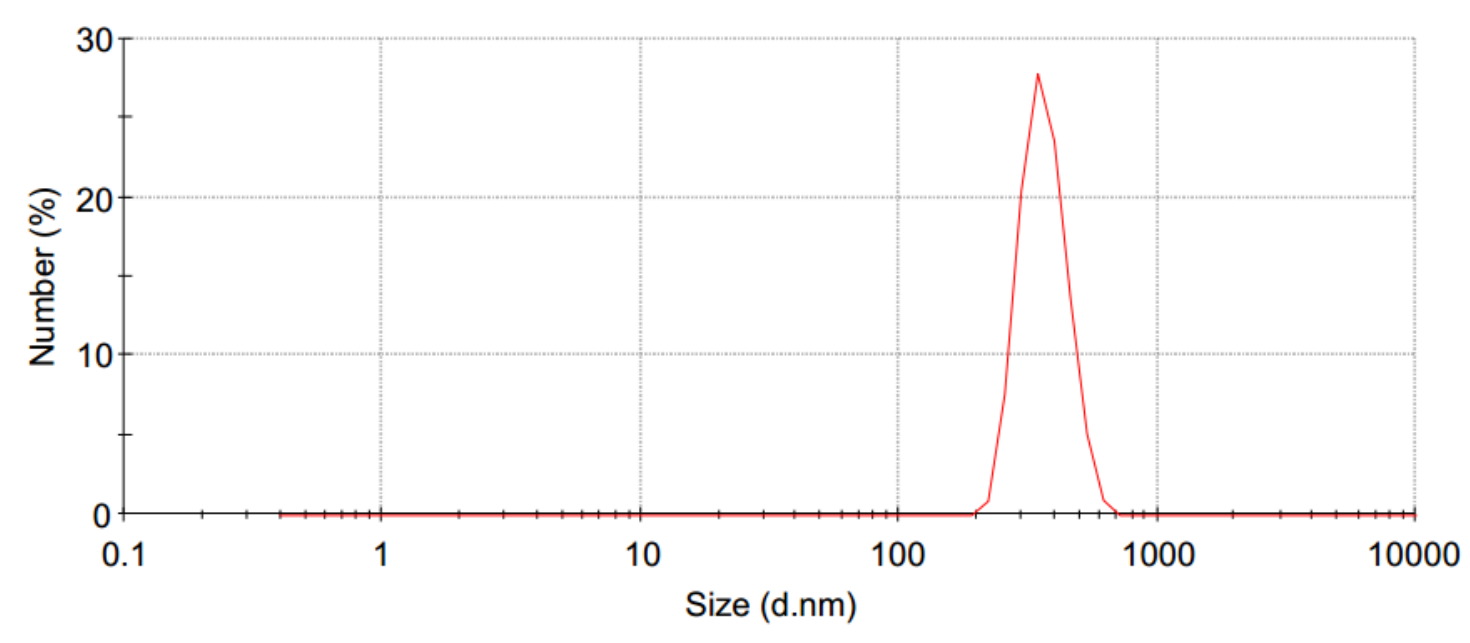




\section{Results $\left(1^{\circ} \mathrm{C}\right.$ for fifth run)}

$\begin{array}{rlllll} & & & \text { Size (d.n... } & \% \text { Number } & \text { Width (d.n... } \\ \text { Z-Average (d.nm): } & 415.0 & \text { Peak 1: } & 397.6 & 100.0 & 88.63 \\ \text { Pdl: } & 0.243 & \text { Peak 2: } & 0.000 & 0.0 & 0.000 \\ \text { Intercept: } & 0.926 & \text { Peak 3: } & 0.000 & 0.0 & 0.000\end{array}$

Result quality Good

Size Distribution by Number

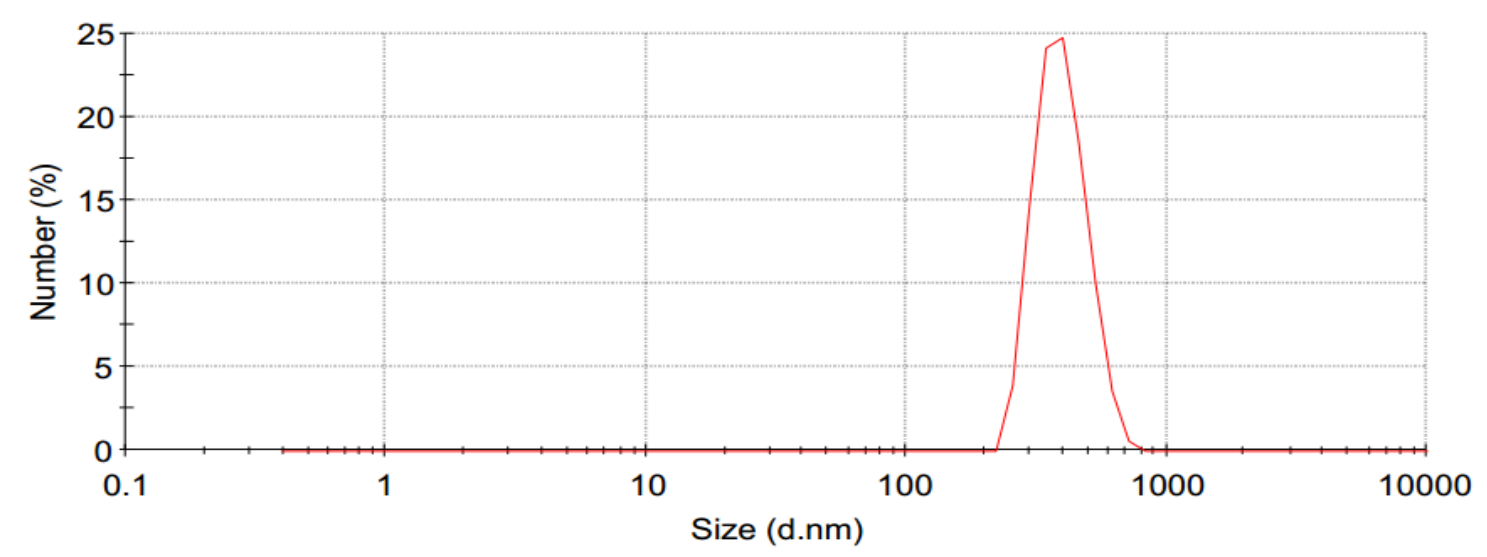

Results $\left(35^{\circ} \mathrm{C}\right.$ for fifth run)

$\begin{array}{rlllll} & & & \text { Size (d.n... } & \text { \% Number } & \text { Width (d.n... } \\ \text { Z-Average (d.nm): } & 515.7 & \text { Peak 1: } & 388.5 & 100.0 & 83.33 \\ \text { Pdl: } & 0.362 & \text { Peak 2: } & 5590 & 0.0 & 579.8 \\ \text { Intercept: } & 0.961 & \text { Peak 3: } & 0.000 & 0.0 & 0.000 \\ \text { Result quality } & \text { Good } & & & & \end{array}$

Size Distribution by Number

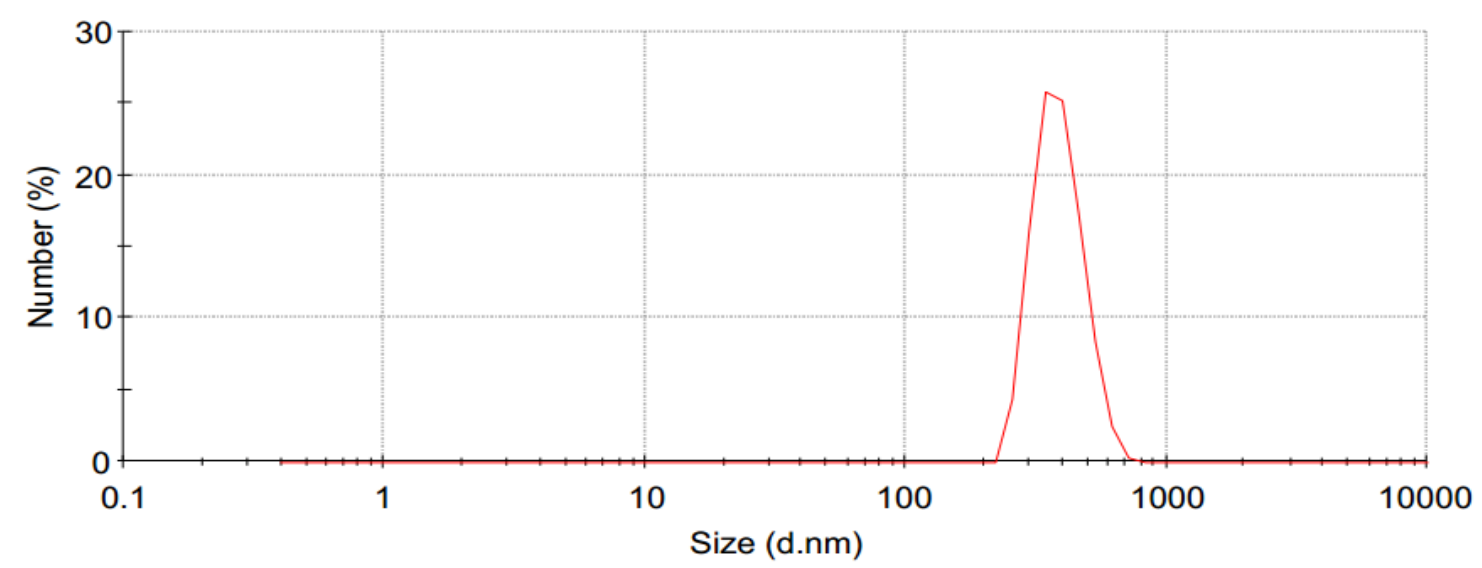

Figure S10. Average hydrodynamic diameters distribution measurement for the recycled catalyst $\mathbf{6}$ as indicated at $15^{\circ} \mathrm{C}$ and at $35^{\circ} \mathrm{C}$. 
Table S1. Investigation of reaction switching in the cascade reaction of 1-phenylprop-2-enone (10a) and aniline (11a) with concomitant transformations of 1-phenyl-3-(phenylamino)propan-1-one $\left(\mathbf{A}^{\prime}\right)$ and chiral product $(S)-1-$ phenyl-3-(phenylamino)propan-1-ol (12a) (the reaction of 1 equivalent of 10a and 1.1 equivalent of $11 \mathrm{a}$ in the presence of catalyst 6 with $2.0 \mathrm{~mol} \%$ of $\mathrm{Ru}$ was carried at $15^{\circ} \mathrm{C}$ for the first $2 \mathrm{~h}$ followed by a continuous proceeding at $35^{\circ} \mathrm{C}$ for the second $6 \mathrm{~h}$ ).

\begin{tabular}{|c|c|c|c|c|c|}
\hline Entry & Catalyst & ${ }^{\circ} \mathrm{C}$ & $\mathrm{h}$ & Yield of $\mathbf{A}^{\prime}(\%)^{b}$ & $\begin{array}{l}\text { Yield (ee) of } \\
\text { 12a }(\%)^{\mathrm{b}}\end{array}$ \\
\hline 1 & Catalyst 6 & 15 & 2 & 93 & ND \\
\hline 2 & Catalyst 6 & 35 & 6 & trace & $93(94)$ \\
\hline 3 & Catalyst 6 & $\begin{array}{l}15 \\
35\end{array}$ & $\begin{array}{l}2 \\
6\end{array}$ & trace & 93 (94) \\
\hline 4 & $\begin{array}{l}\text { Catalyst } \mathbf{2}+\text { acrylamide and } \\
\text { acrylonitrile }\end{array}$ & $\begin{array}{l}15 \\
35\end{array}$ & $\begin{array}{l}2 \\
6\end{array}$ & 6 & $85(91)$ \\
\hline 5 & $\begin{array}{l}\text { Cp*RuTsDPEN } \\
\text { acrylamide and acrylonitrile }\end{array}+$ & $\begin{array}{l}15 \\
35\end{array}$ & $\begin{array}{l}2 \\
6\end{array}$ & 14 & 79 (86) \\
\hline
\end{tabular}

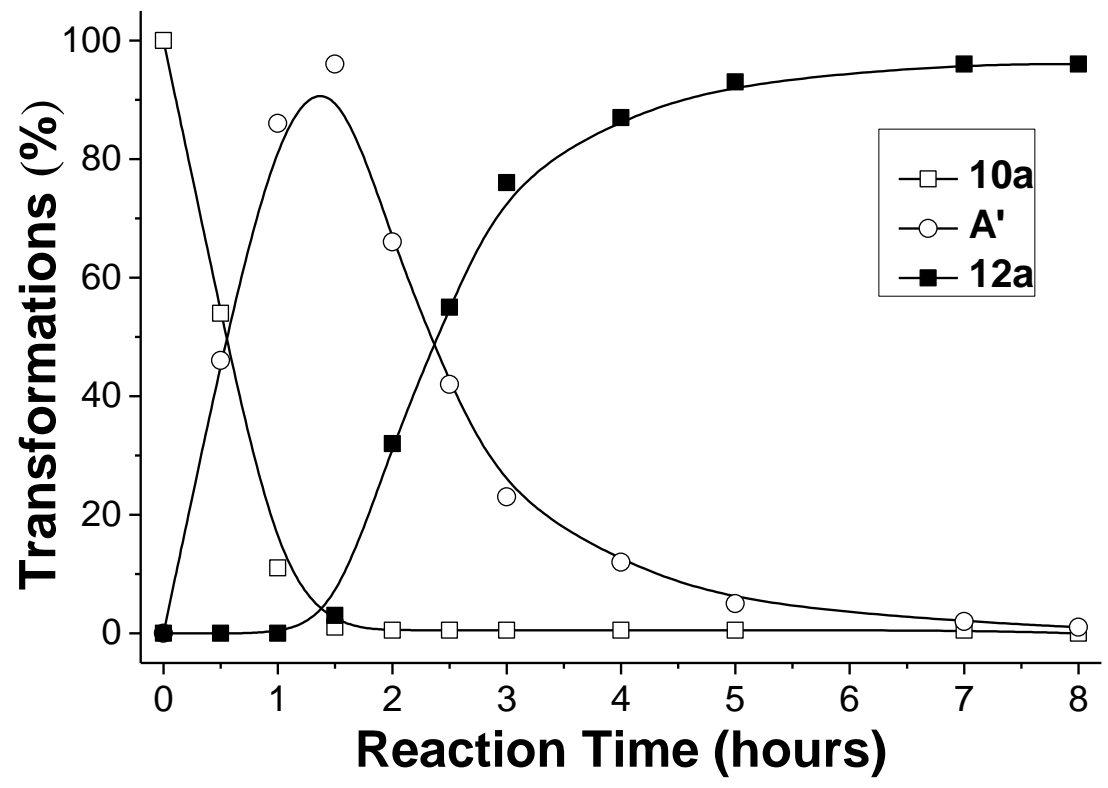

Figure S11. Time course in the 6-catalyzed transformation of 1-phenylprop-2-enone (10a) and aniline (11a) with concomitant transformation of 1-phenyl-3-(phenylamino)propan-1-one ( $\left.\mathbf{A}^{\prime}\right)$ (the reaction of 1 equivalent of $\mathbf{1 0 a}$ and 1.1 equivalent of $11 \mathbf{a}$ in the presence of catalyst $\mathbf{6}$ with $2.0 \mathrm{~mol} \%$ of Ru was carried at $15{ }^{\circ} \mathrm{C}$ for the first $2 \mathrm{~h}$ followed by a continuous proceeding at $35^{\circ} \mathrm{C}$ for the second $6 \mathrm{~h}$ ). 
(S)-4-phenylacetophenol (9a): (HPLC: Chiracel AD-H, detected at $254 \mathrm{~nm}$, eluent: n-hexane $/ 2-$ propanol $=97 / 3$, flow rate $=1.0 \mathrm{~mL} / \mathrm{min}, 25^{\circ} \mathrm{C}$ ).
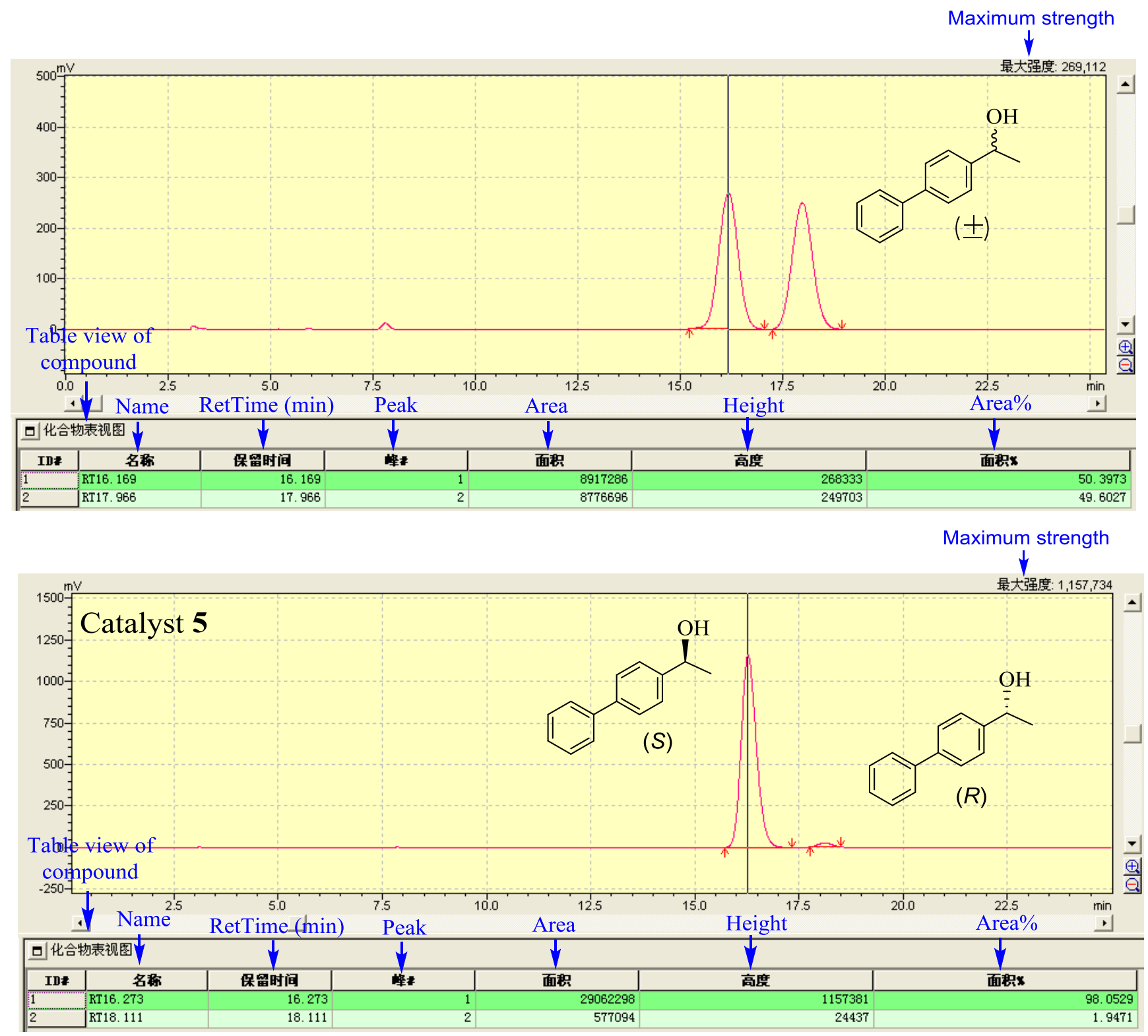

\section{Translation of Chinese into English as follows.}

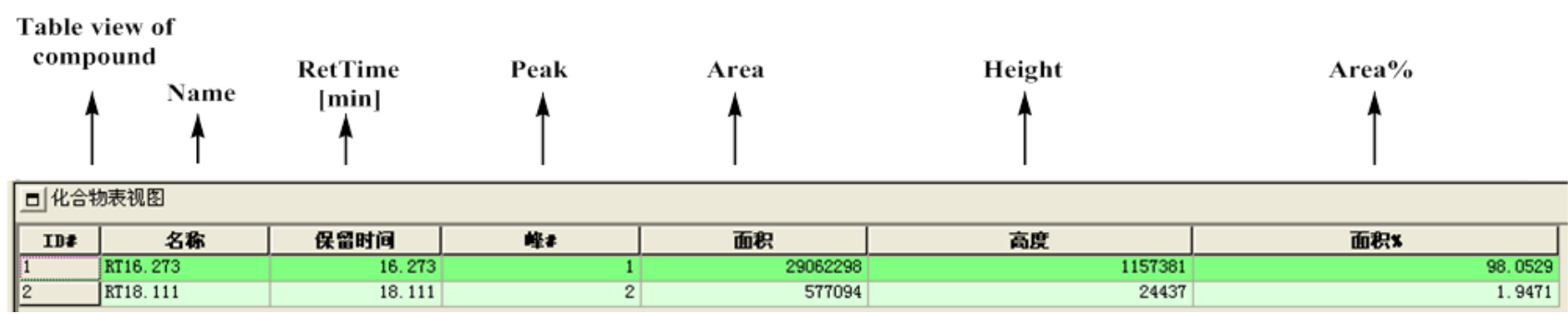


(S)-1-(4-(4-floro)phenyl)ethanol (9b): (HPLC: Chiracel AD-H, detected at $254 \mathrm{~nm}$, eluent: n-hexane $/ 2-$ propanol $=97 / 3$, flow rate $=1.0 \mathrm{~mL} / \mathrm{min}, 25^{\circ} \mathrm{C}$ ).
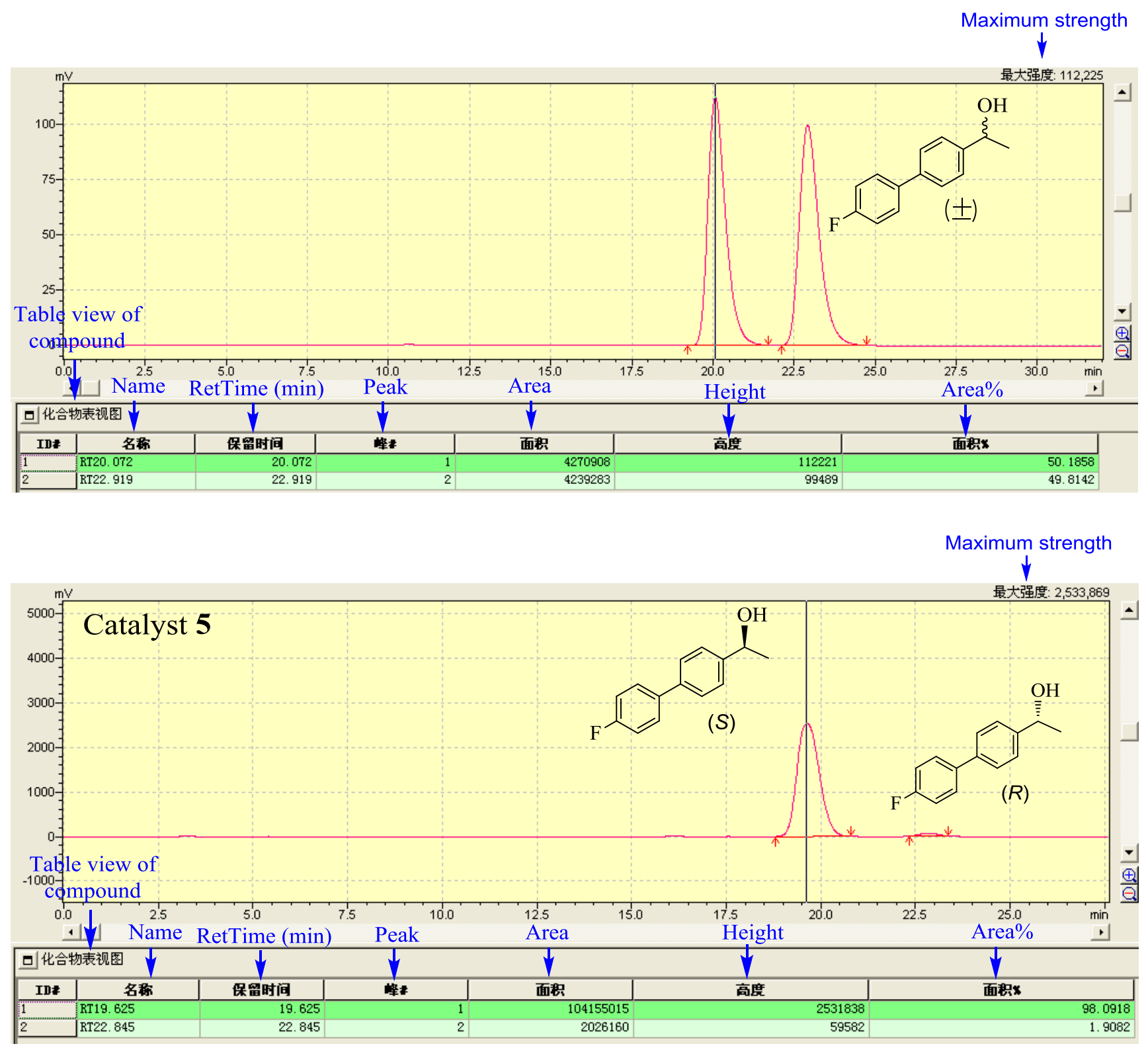

$\underline{\text { Translation of Chinese into English as follows. }}$

\begin{tabular}{|c|c|c|c|c|c|c|}
\hline \multicolumn{2}{|c|}{$\begin{array}{l}\text { Table view of } \\
\text { compound }\end{array}$} & Ret Time & $\begin{array}{c}\text { Peak } \\
1\end{array}$ & Area & Height & Area $\%$ \\
\hline \multicolumn{7}{|c|}{ 므化合物表视圈 } \\
\hline IDE & 名称 & 保咍时间 & 崔 & 面积 & 高度 & 面短 $x$ \\
\hline 1 & RT16.273 & 16.273 & 1 & 29062298 & 1157381 & 98.0529 \\
\hline 2 & RT18.111 & 18.111 & 2 & 577094 & 24437 & 1.9471 \\
\hline
\end{tabular}


(S)-1-(4-(4-chloro)phenyl)ethanol (9c): (HPLC: Chiracel AD-H, detected at $254 \mathrm{~nm}$, eluent: $\mathrm{n}$-hexane $/ 2-$ propanol $=97 / 3$, flow rate $=1.0 \mathrm{~mL} / \mathrm{min}, 25^{\circ} \mathrm{C}$ ).
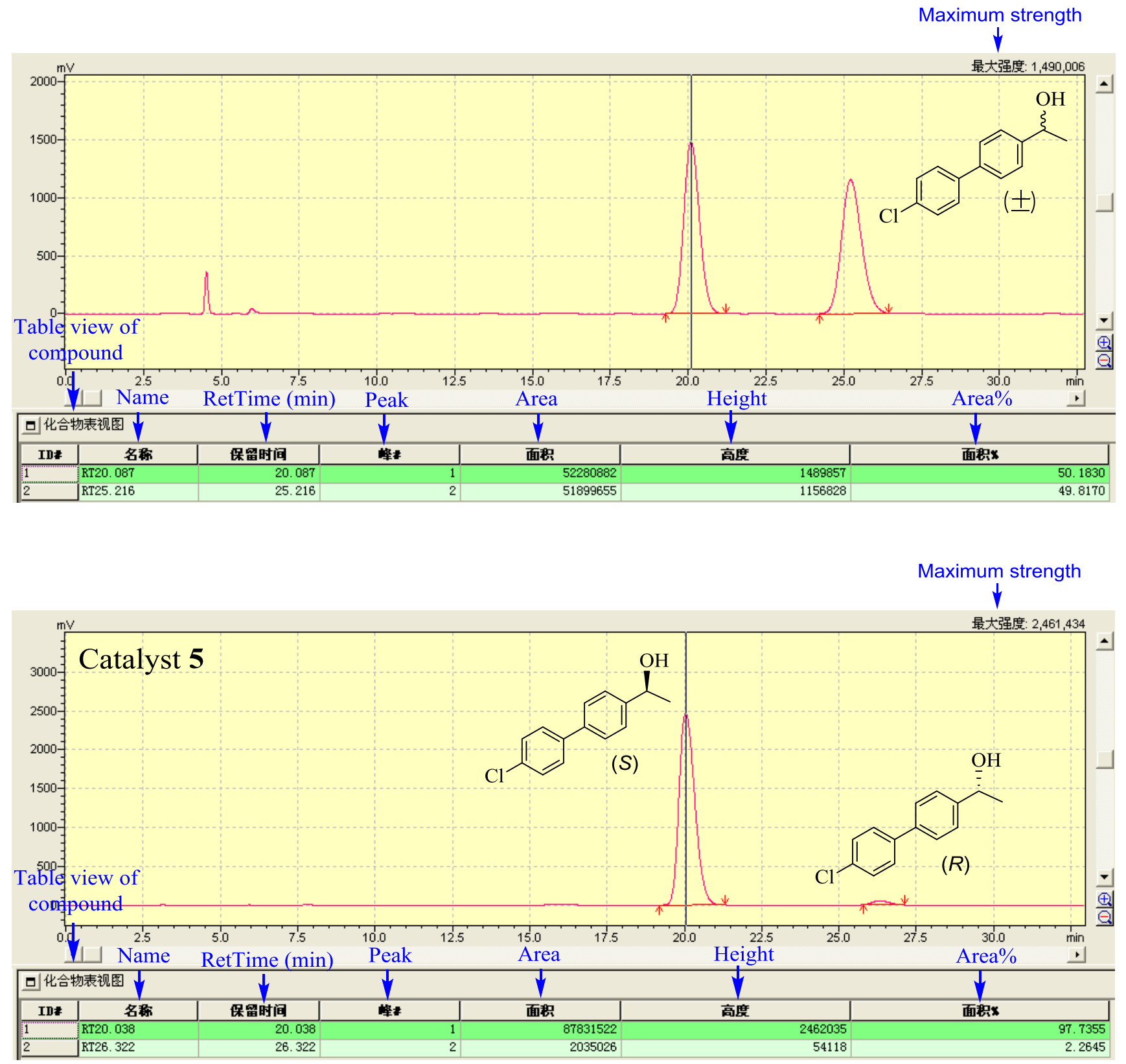

Translation of Chinese into English as follows.

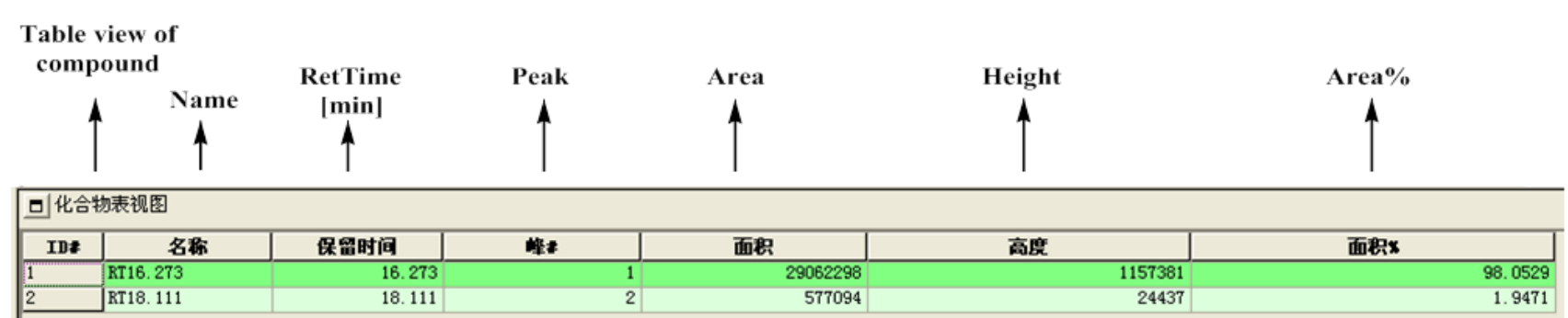


(S)-1-(4-(3-chloro)phenyl)ethanol (9d): (HPLC: Chiracel AD-H, detected at $254 \mathrm{~nm}$, eluent: $\mathrm{n}$-hexane $/ 2-$ propanol $=79 / 1$, flow rate $=0.8 \mathrm{~mL} / \mathrm{min}, 25^{\circ} \mathrm{C}$ ).

Maximum strength

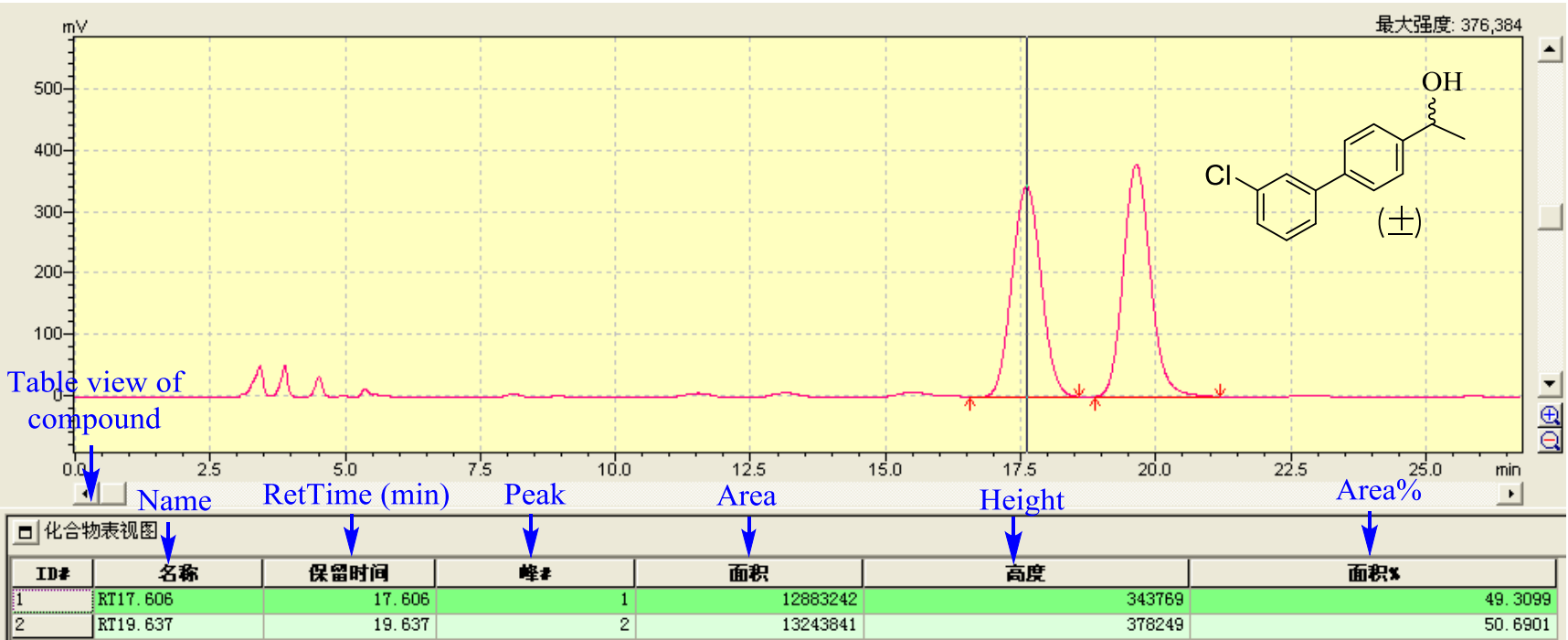

Maximum strength

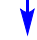

最大强度: $2,837,57$

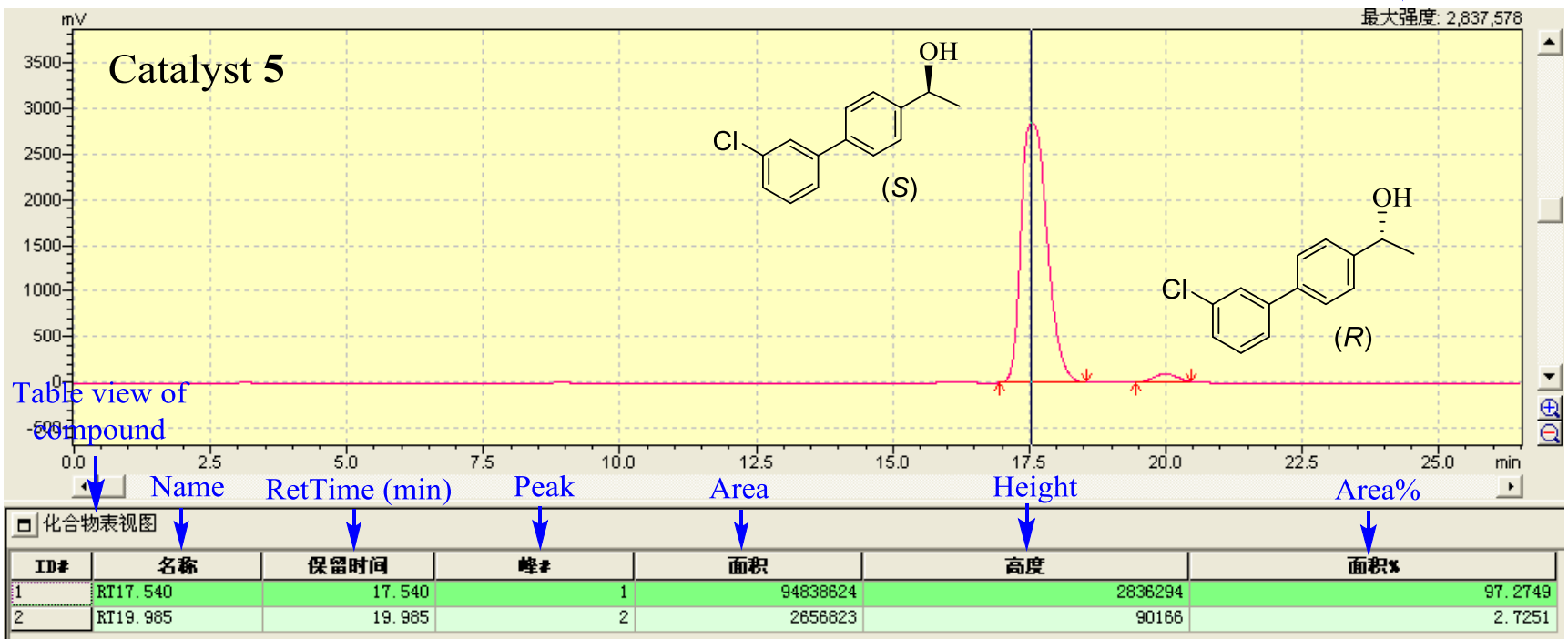

Translation of Chinese into English as follows.

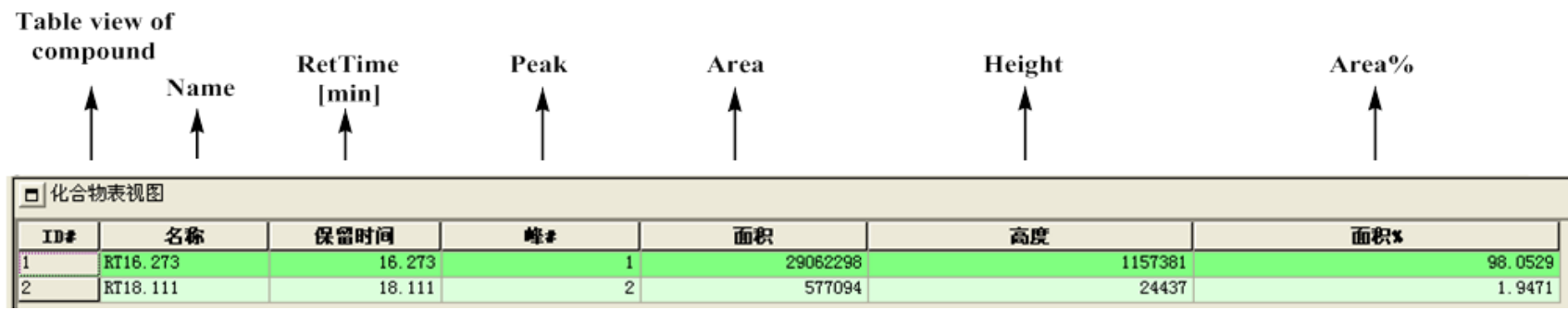


(S)-1-(4-(4-trifluoromethyl)phenyl)ethanol (9e): (HPLC: Chiracel AD-H, detected at $254 \mathrm{~nm}$, eluent: $\mathrm{n}$-hexane $/ 2-$ propanol $=97 / 3$, flow rate $=1.0 \mathrm{~mL} / \mathrm{min}, 25^{\circ} \mathrm{C}$ ).

Maximum strength

$$
\downarrow
$$
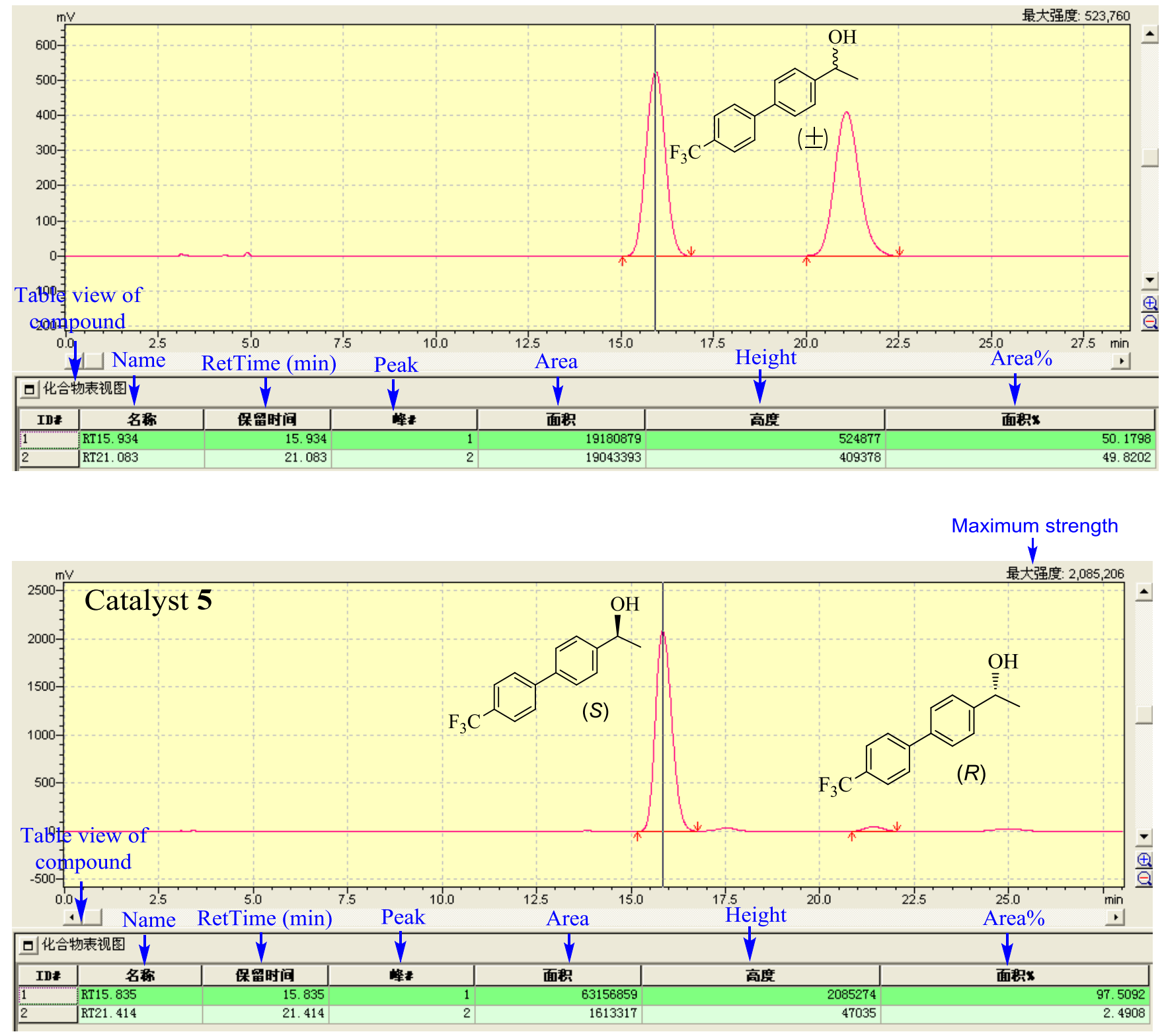

Translation of Chinese into English as follows.

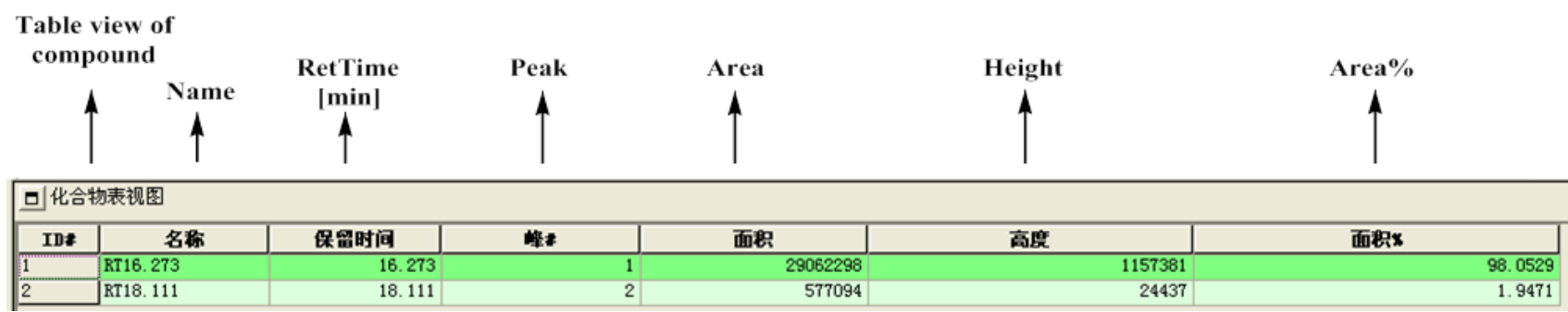


(S)-1-(4-(3-trifluouomethyl)phenyl)ethanol (9f): (HPLC: Chiracel AD-H, detected at $254 \mathrm{~nm}$, eluent: $\mathrm{n}$-hexane $/ 2-$ propanol $=79 / 1$, flow rate $=0.8 \mathrm{~mL} / \mathrm{min}, 25^{\circ} \mathrm{C}$ ).
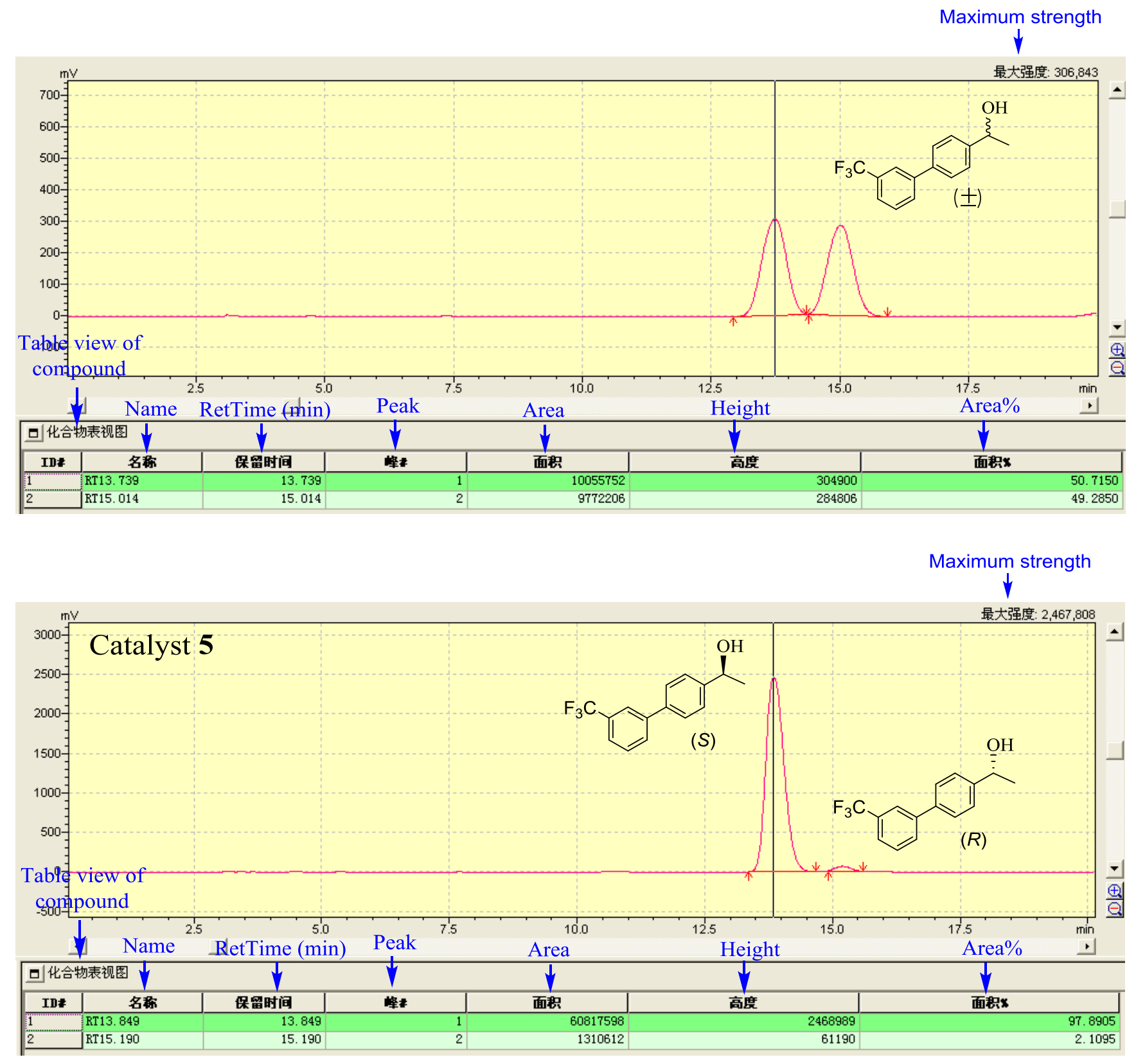

\section{Translation of Chinese into English as follows.}

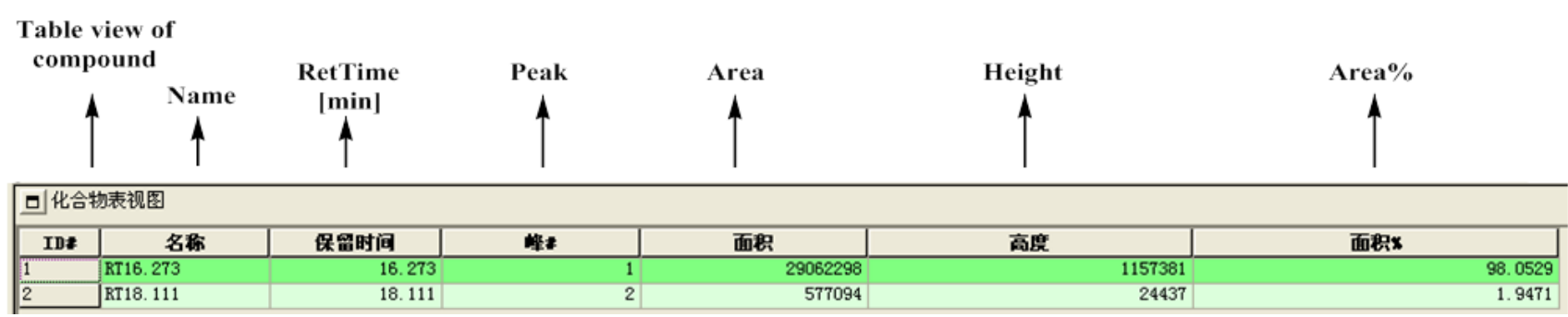


(S)-1-(4-(4-methyl)phenyl)ethanol (9g): (HPLC: Chiracel AD-H, detected at $254 \mathrm{~nm}$, eluent: $\mathrm{n}$-hexane $/ 2-$ propanol $=97 / 3$, flow rate $=1.0 \mathrm{~mL} / \mathrm{min}, 25^{\circ} \mathrm{C}$ ).
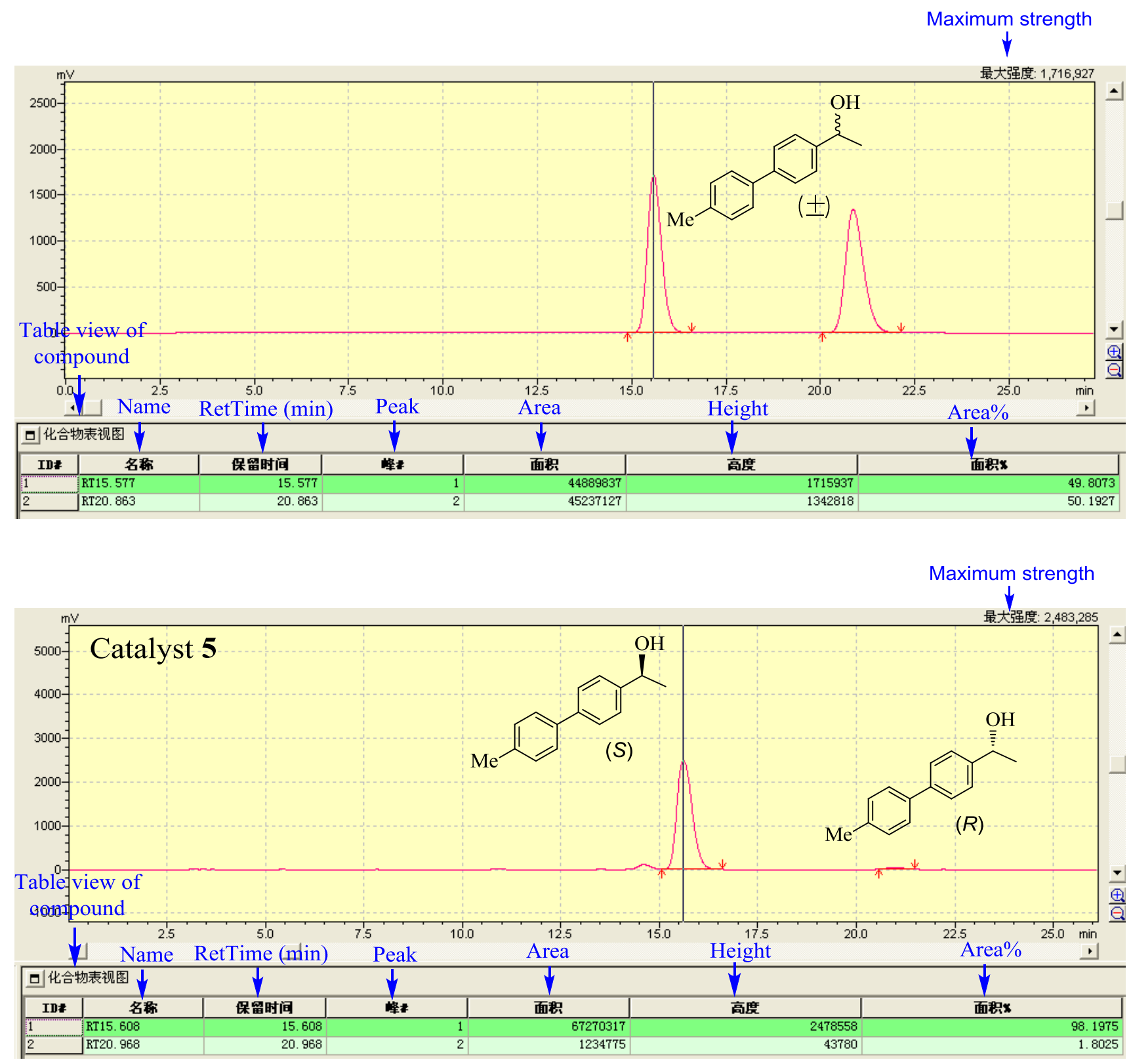

Translation of Chinese into English as follows.

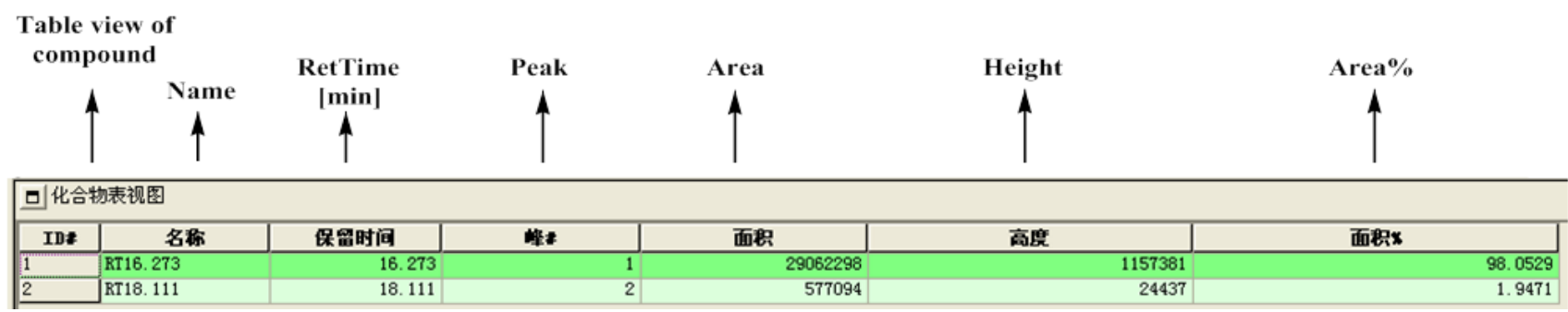


(S)-1-(4-(3-methyl)phenyl)ethanol (9h): (HPLC: Chiracel AD-H, detected at $254 \mathrm{~nm}$, eluent: $\mathrm{n}$-hexane $/ 2-$ propanol $=79 / 1$, flow rate $=0.8 \mathrm{~mL} / \mathrm{min}, 25^{\circ} \mathrm{C}$ ).
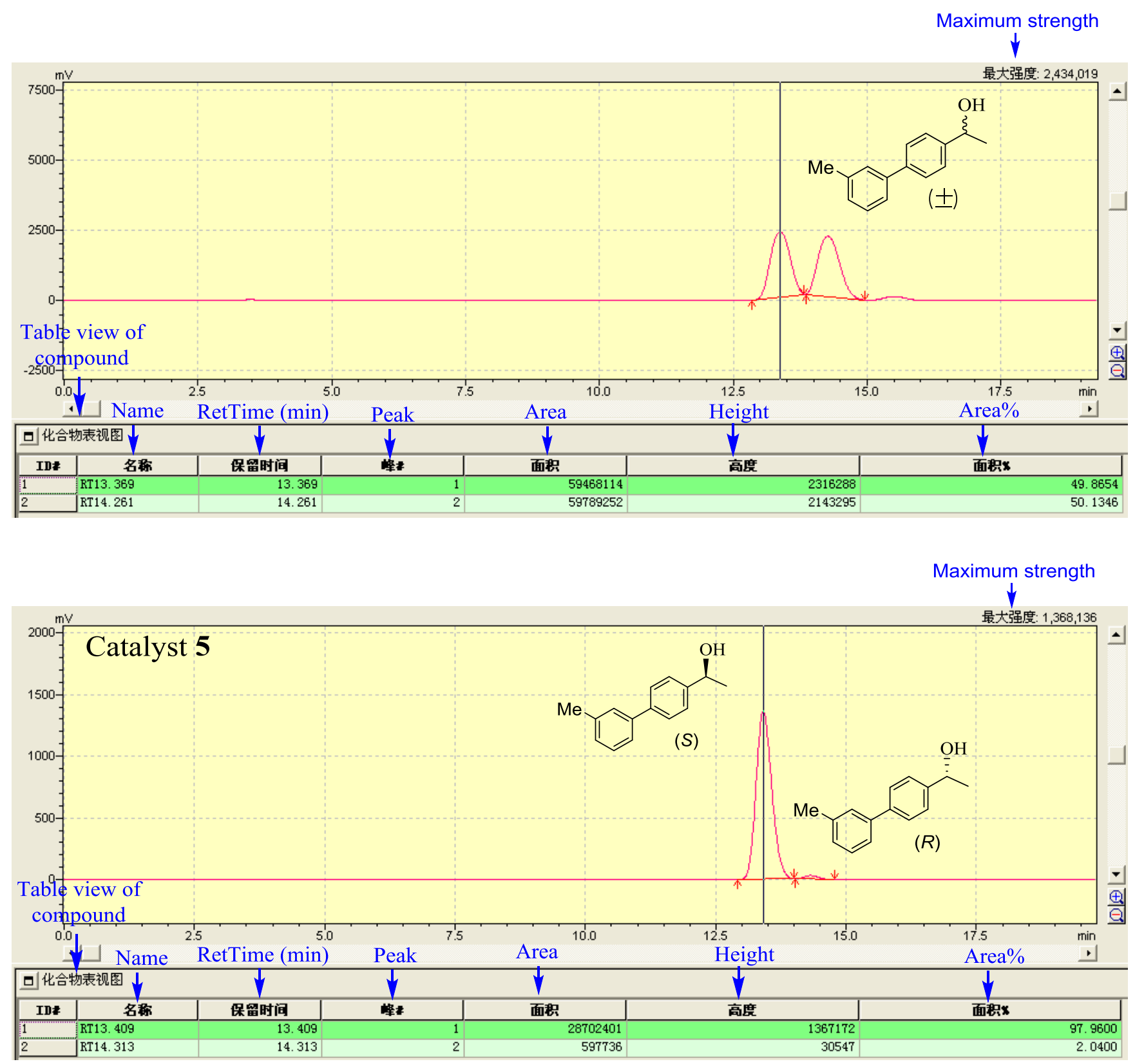

Translation of Chinese into English as follows.

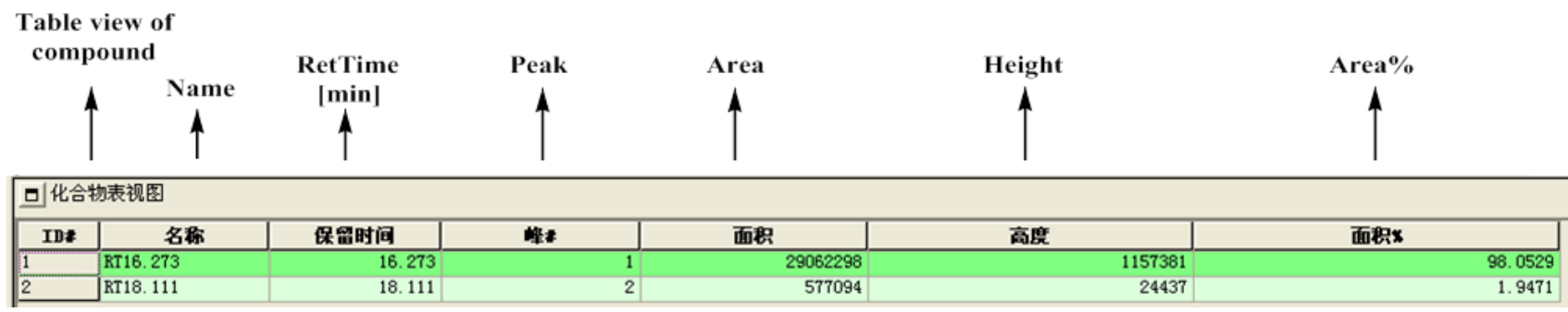


(S)-1-(4'-methoxy-[1,1'-biphenyl]-4-yl)ethan-1-ol (9i): (HPLC: Chiracel AD-H, detected at 254 nm, eluent: $n-$ hexane $/ 2-$ propanol $=97 / 3$, flow rate $=1.0 \mathrm{~mL} / \mathrm{min}, 25^{\circ} \mathrm{C}$ ).
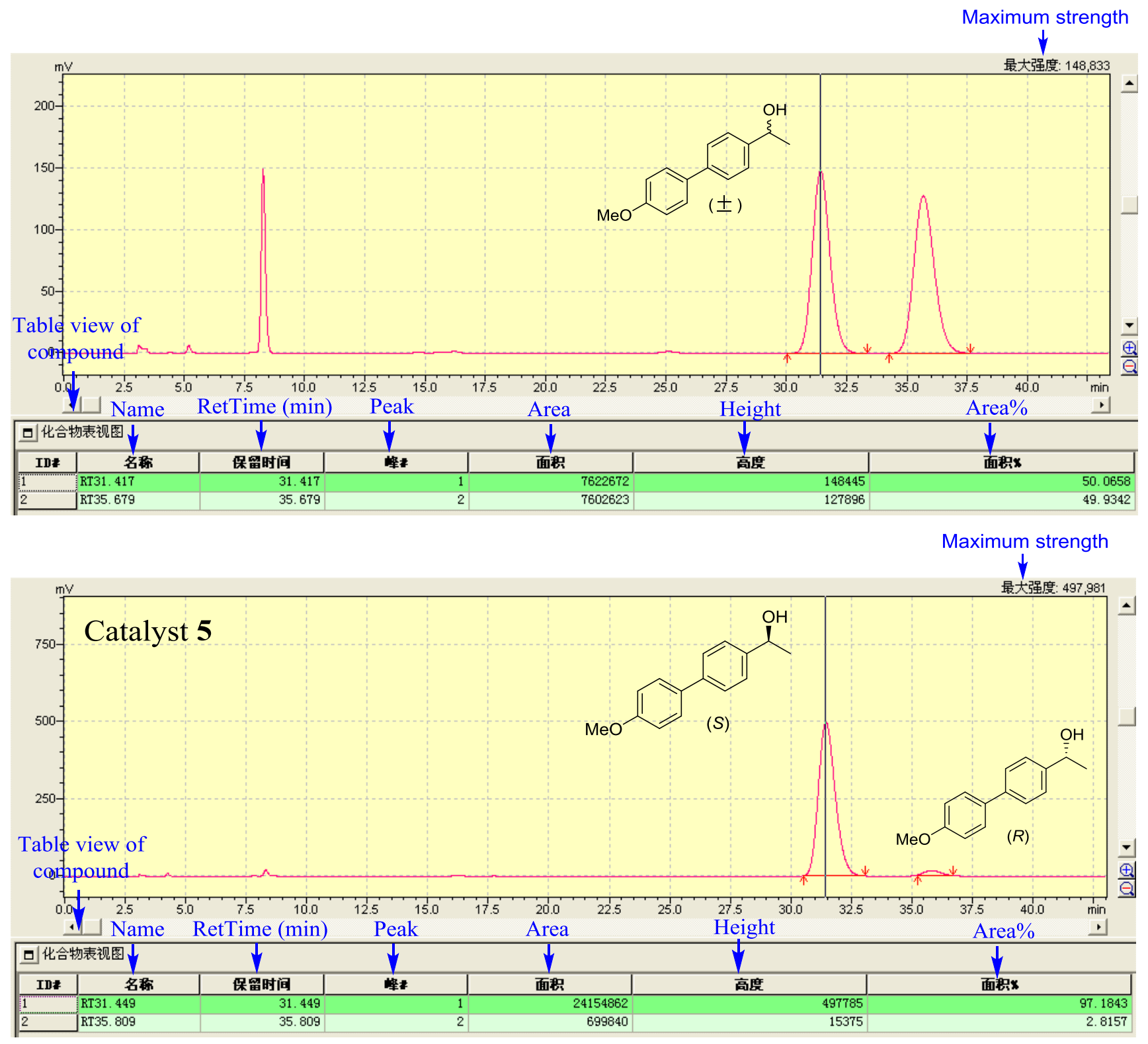

\section{Translation of Chinese into English as follows.}

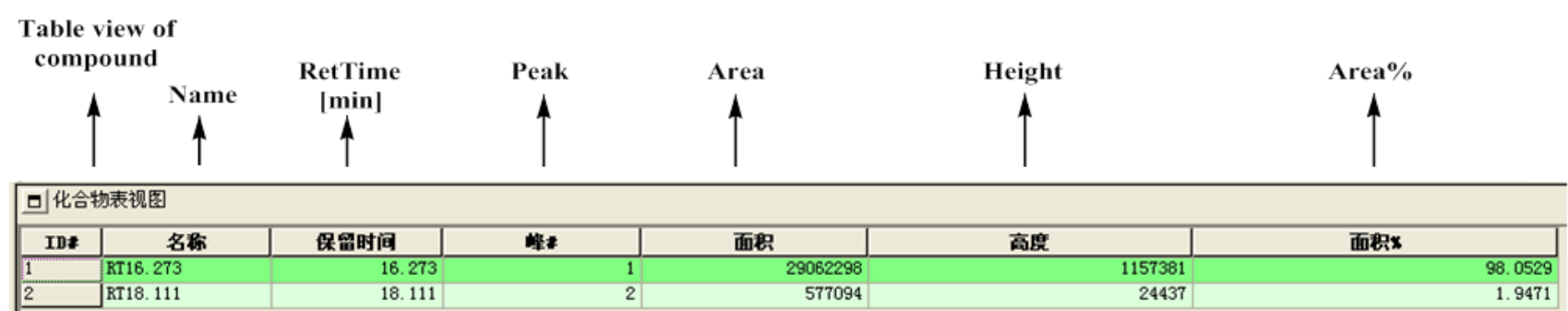


(S)-1-(4-(thiophen-3-yl)phenyl)ethanol (9j): (HPLC: Chiracel AD-H, detected at 254 nm, eluent: nhexane $/ 2$-propanol $=97 / 3$, flow rate $=1.0 \mathrm{~mL} / \mathrm{min}, 25^{\circ} \mathrm{C}$ ).
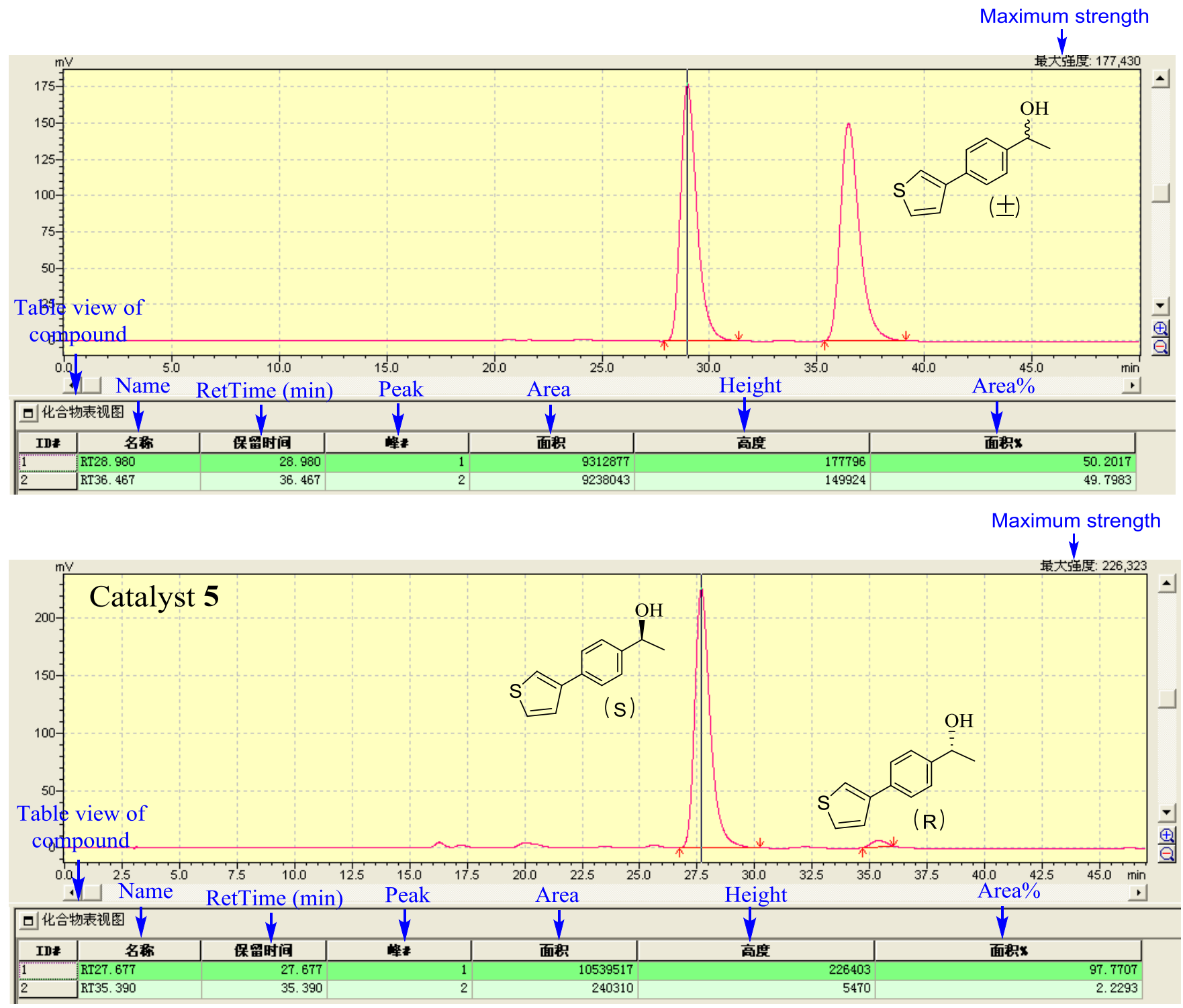

Translation of Chinese into English as follows.

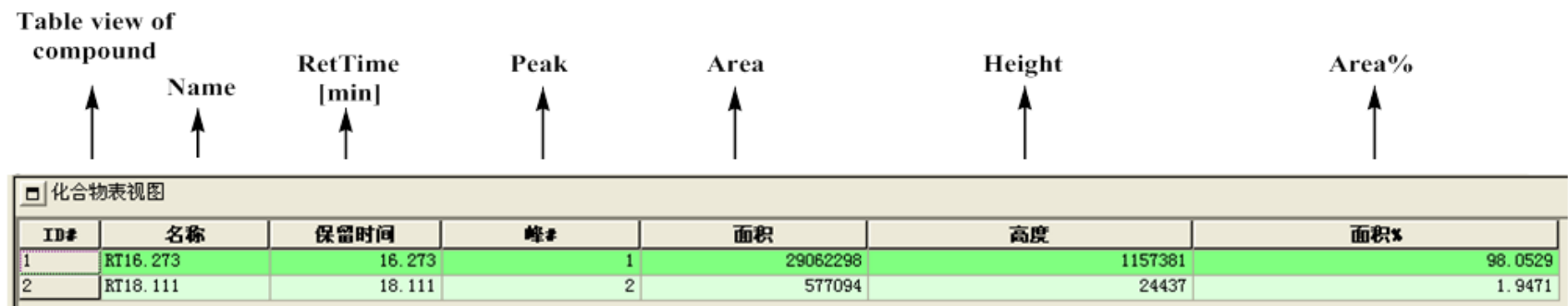


(S)-1-(4-(pyridin-4-yl)phenyl)ethanol (9k): (HPLC: Chiracel OD-H, detected at 254 nm, eluent: nhexane $/ 2$-propanol $=85 / 15$, flow rate $=0.8 \mathrm{~mL} / \mathrm{min}, 25^{\circ} \mathrm{C}$ ).
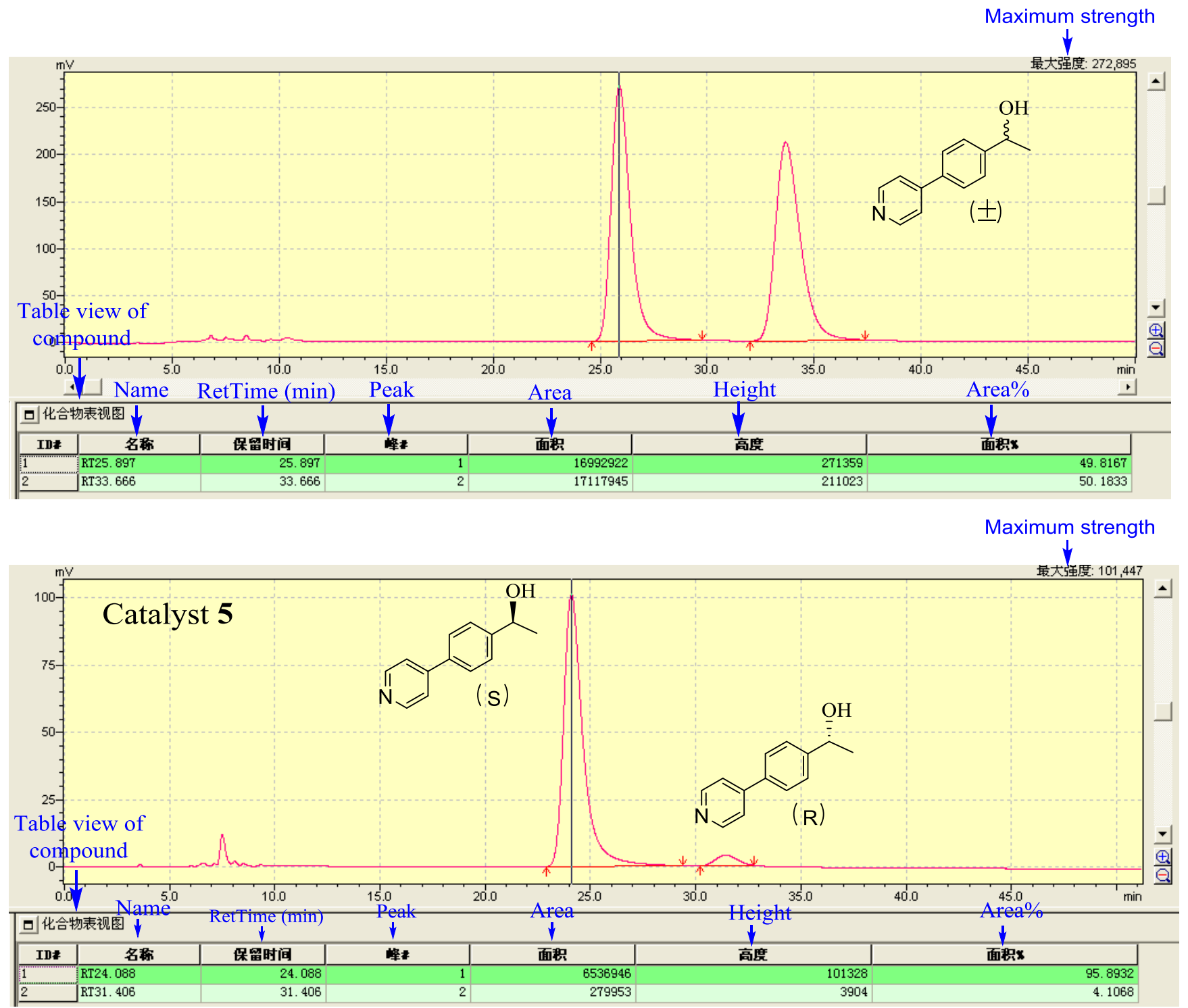

Translation of Chinese into English as follows.

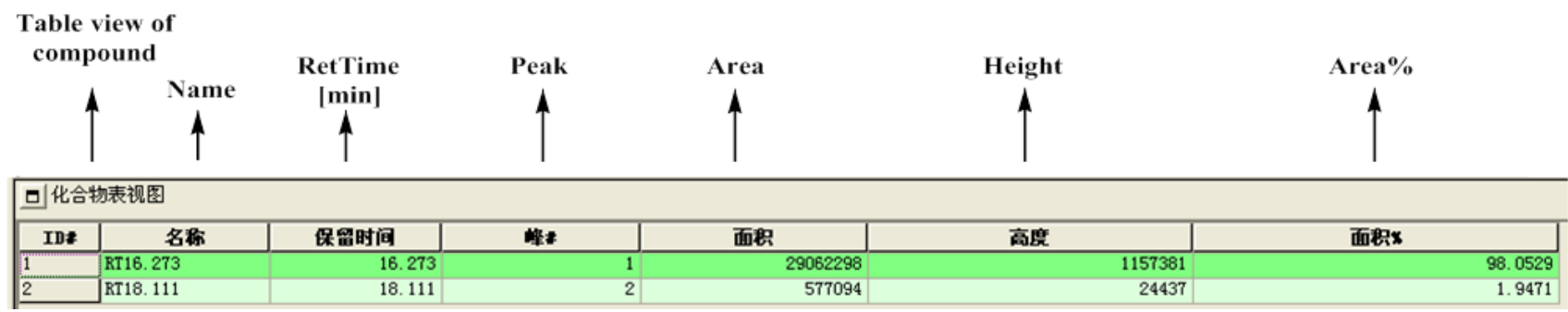


(S)-3-phenylacetophenol (91): (HPLC: Chiracel AD-H, detected at $254 \mathrm{~nm}$, eluent: $\mathrm{n}$-hexane $/ 2-$ propanol $=97 / 3$, flow rate $=1.0 \mathrm{~mL} / \mathrm{min}, 25^{\circ} \mathrm{C}$ ).
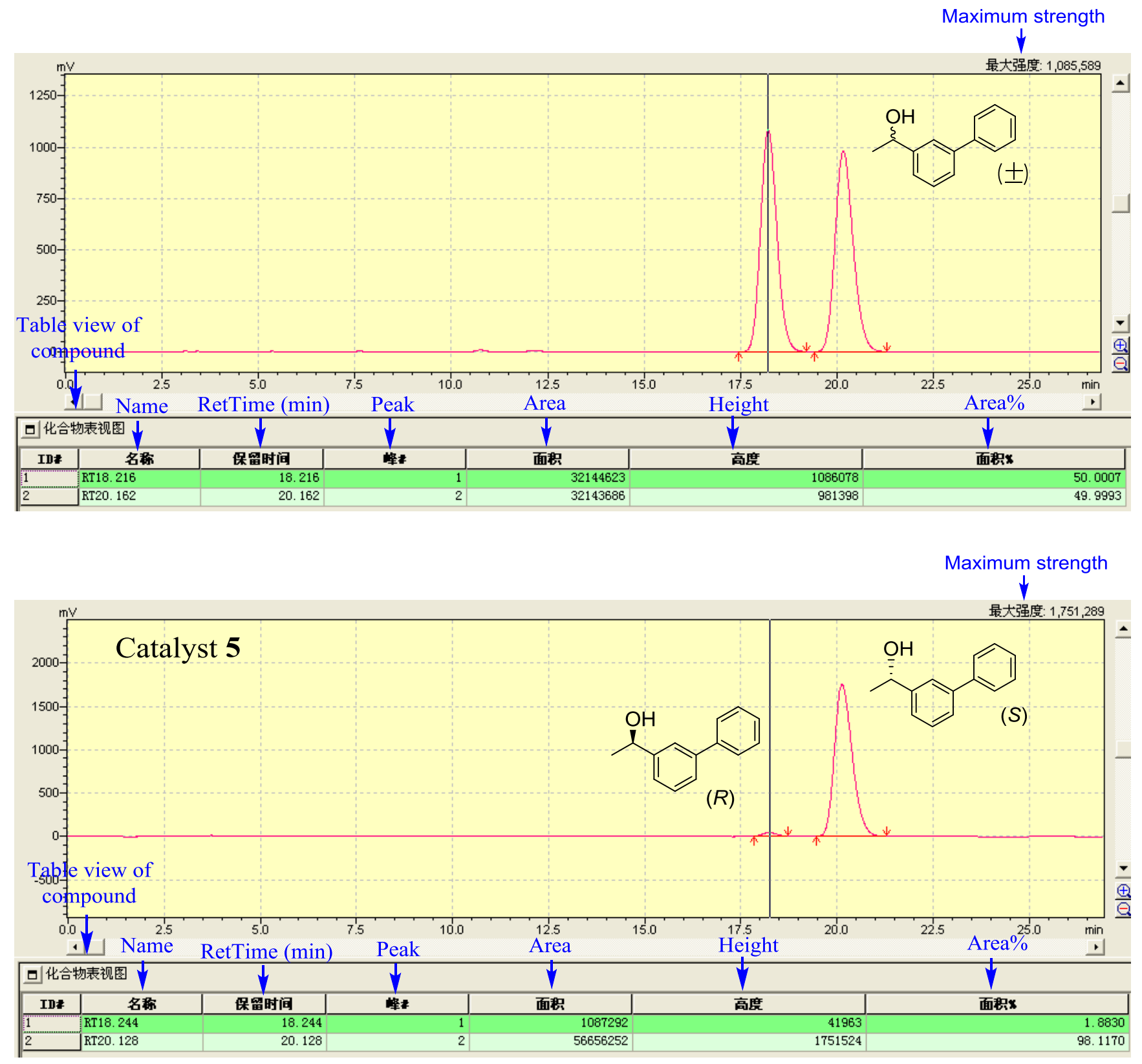

\section{Translation of Chinese into English as follows.}

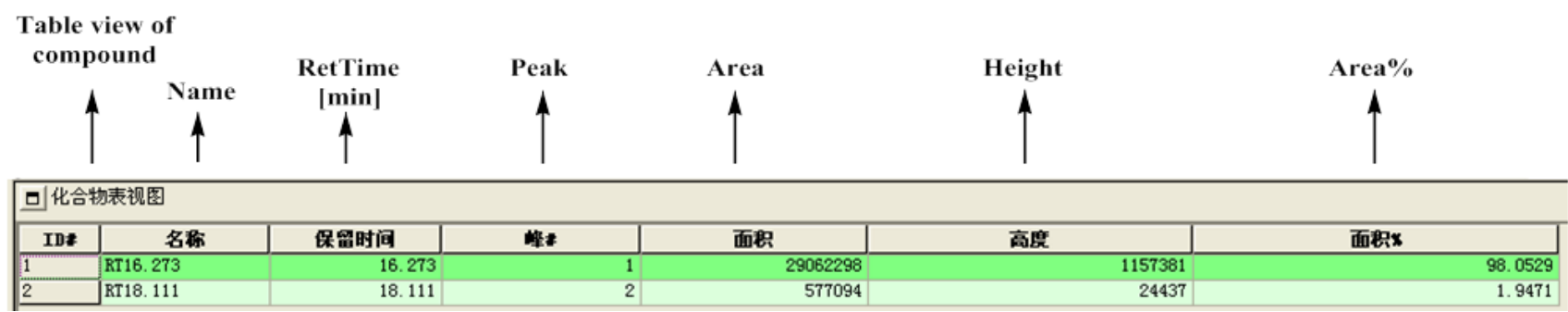


(S)-1-(3-(4-fluoro)phenyl)ethanol (9m): (HPLC: Chiracel AD-H, detected at $254 \mathrm{~nm}$, eluent: $\mathrm{n}$-hexane $/ 2-$ propanol $=97 / 3$, flow rate $=1.0 \mathrm{~mL} / \mathrm{min}, 25^{\circ} \mathrm{C}$ ).
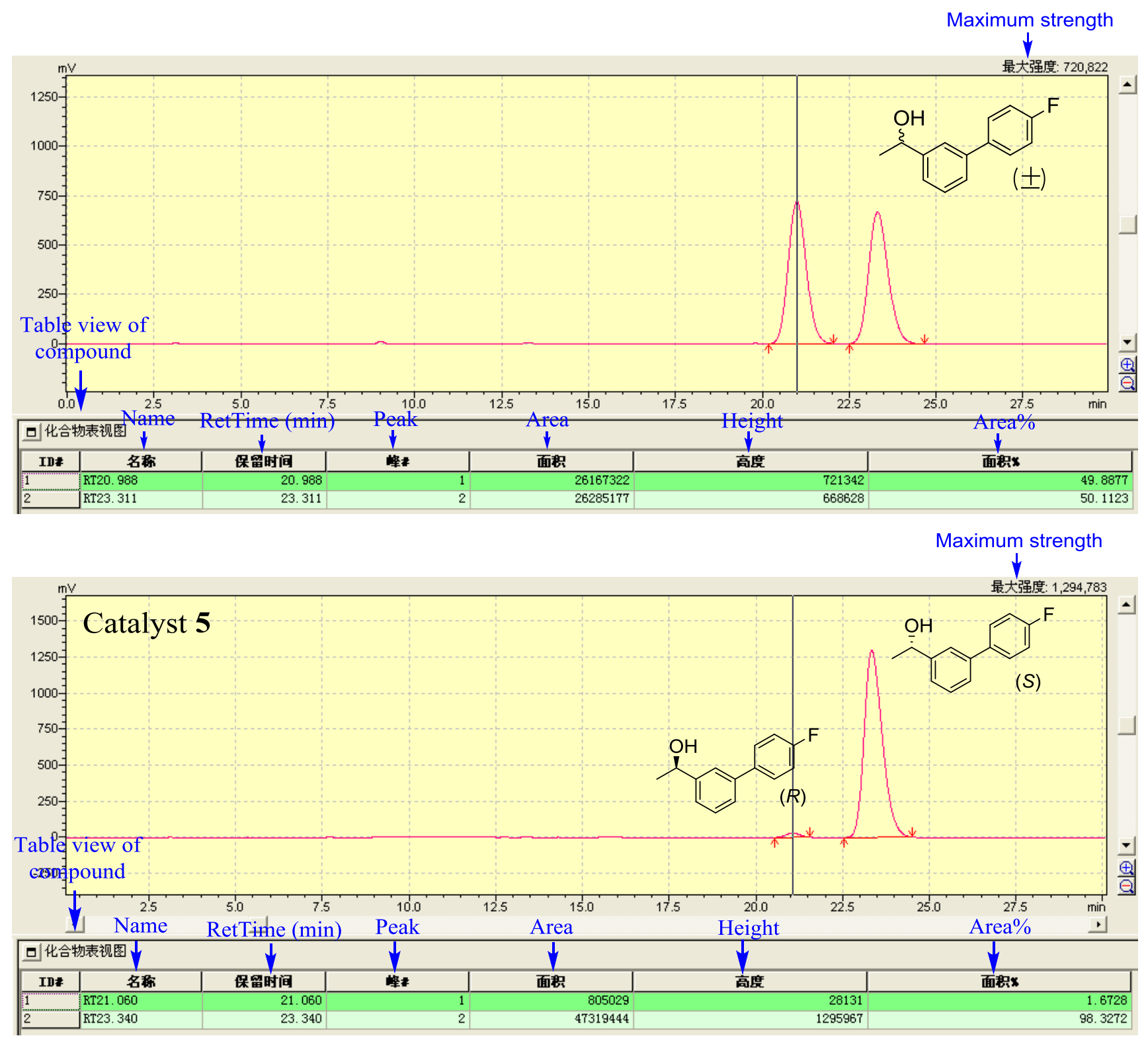

Translation of Chinese into English as follows.

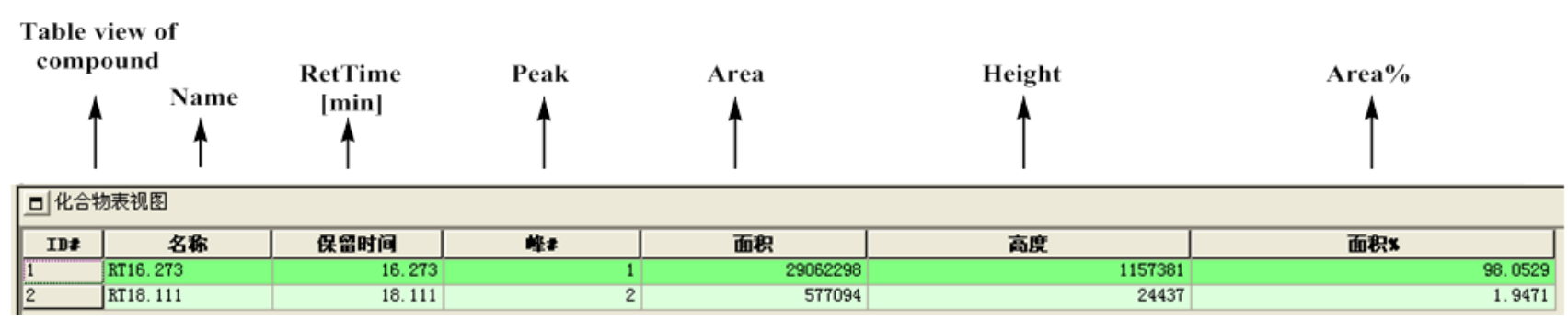


(S)-1-(3-(4- chloro)phenyl)ethanol (9n): (HPLC: Chiracel AD-H, detected at $254 \mathrm{~nm}$, eluent: $\mathrm{n}$-hexane $/ 2-$ propanol $=97 / 3$, flow rate $=1.0 \mathrm{~mL} / \mathrm{min}, 25^{\circ} \mathrm{C}$ ).
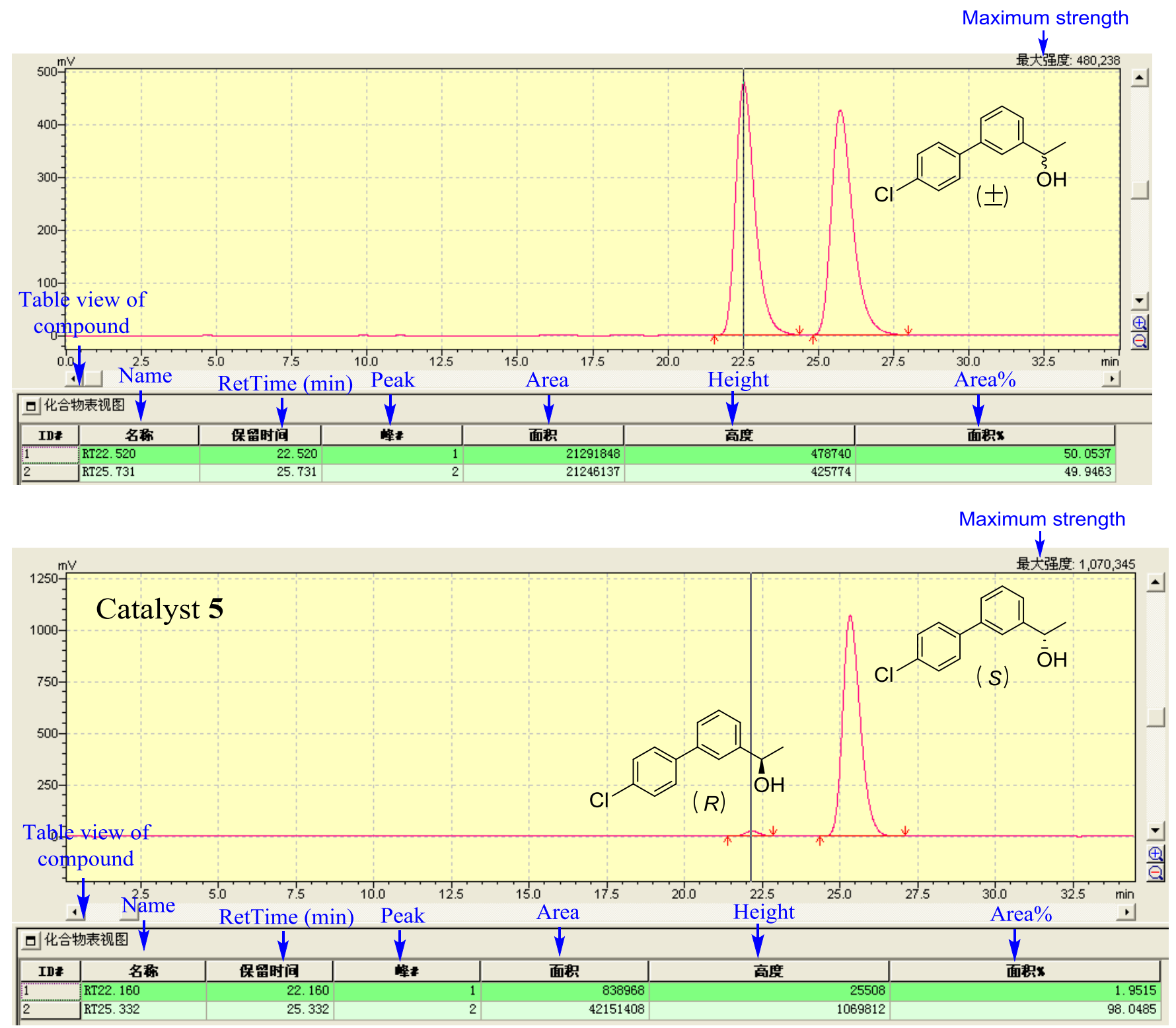

\section{Translation of Chinese into English as follows.}

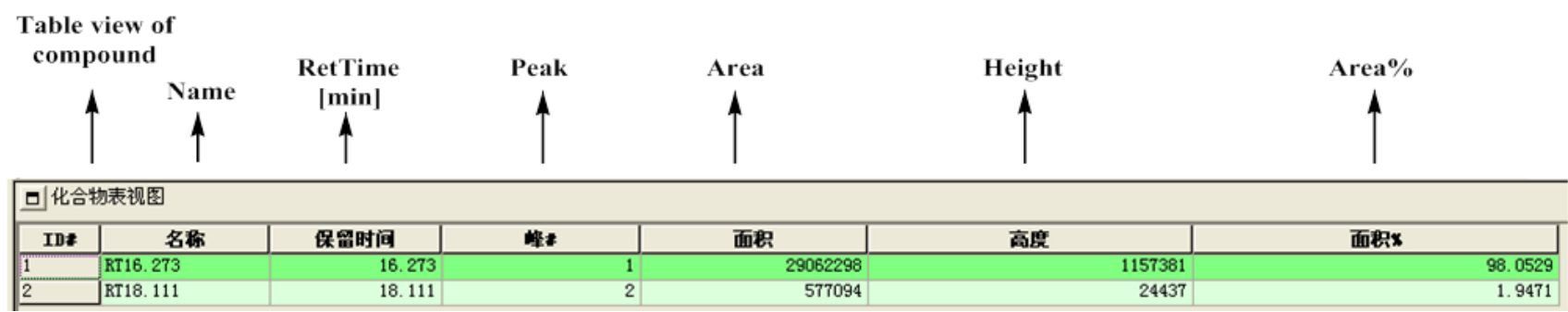


(S)-1-(3-(3-chloro)phenyl)ethanol (9o): (HPLC: Chiracel AD-H, detected at $254 \mathrm{~nm}$, eluent: $\mathrm{n}$-hexane $/ 2-$ propanol $=97 / 3$, flow rate $=1.0 \mathrm{~mL} / \mathrm{min}, 25^{\circ} \mathrm{C}$ ).
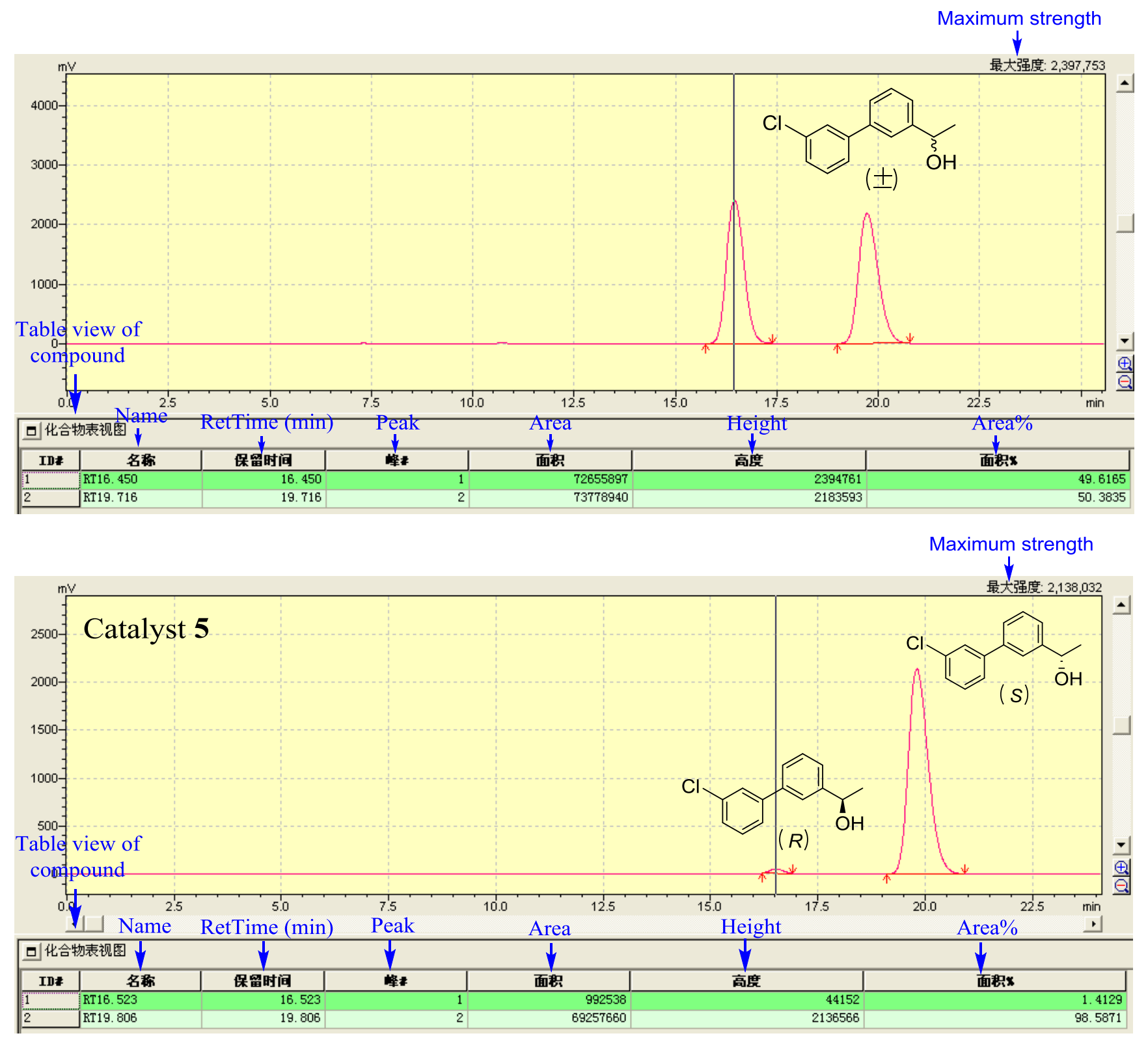

\section{Translation of Chinese into English as follows.}

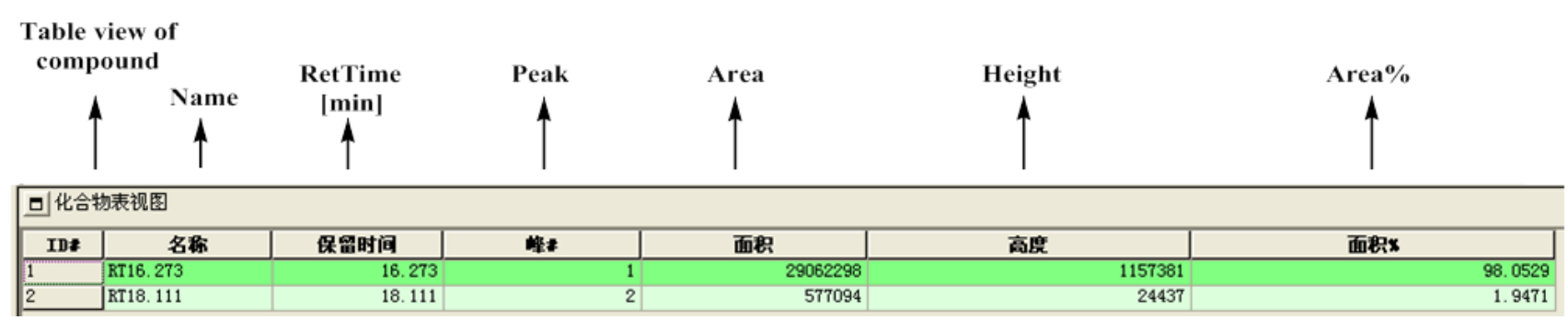


(S)-1-(3-(3-trifluouomethyl)phenyl)ethanol (9p): (HPLC: Chiracel AD-H, detected at $254 \mathrm{~nm}$, eluent: $\mathrm{n}$-hexane $/ 2-$ propanol $=97 / 3$, flow rate $=1.0 \mathrm{~mL} / \mathrm{min}, 25^{\circ} \mathrm{C}$ ).
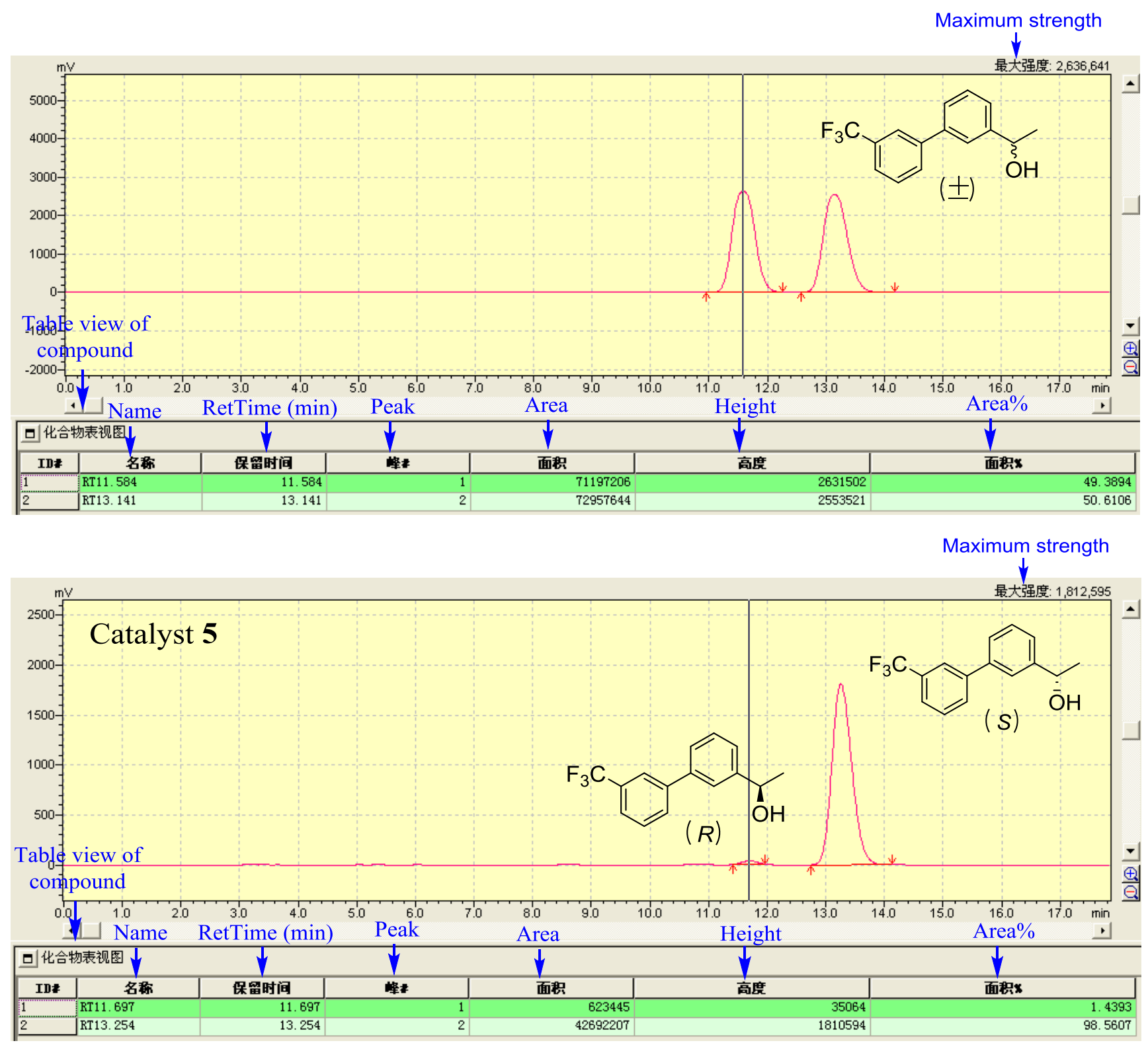

Translation of Chinese into English as follows.

Table view of
compound


(S)-1-(3-(4-methyl)phenyl)ethanol (9q): (HPLC: Chiracel AD-H, detected at $254 \mathrm{~nm}$, eluent: n-hexane $/ 2-$ propanol $=97 / 3$, flow rate $=1.0 \mathrm{~mL} / \mathrm{min}, 25^{\circ} \mathrm{C}$ ).

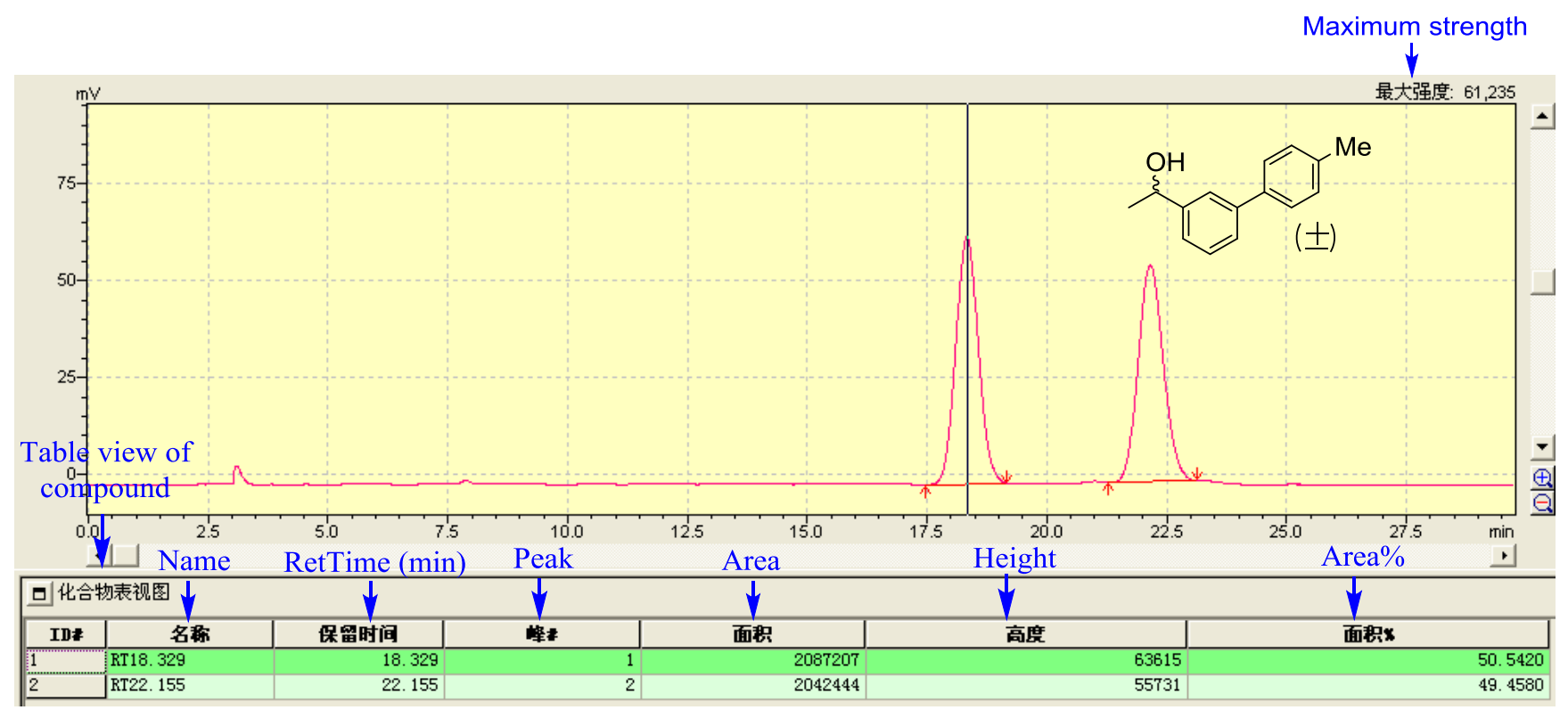

Maximum strength

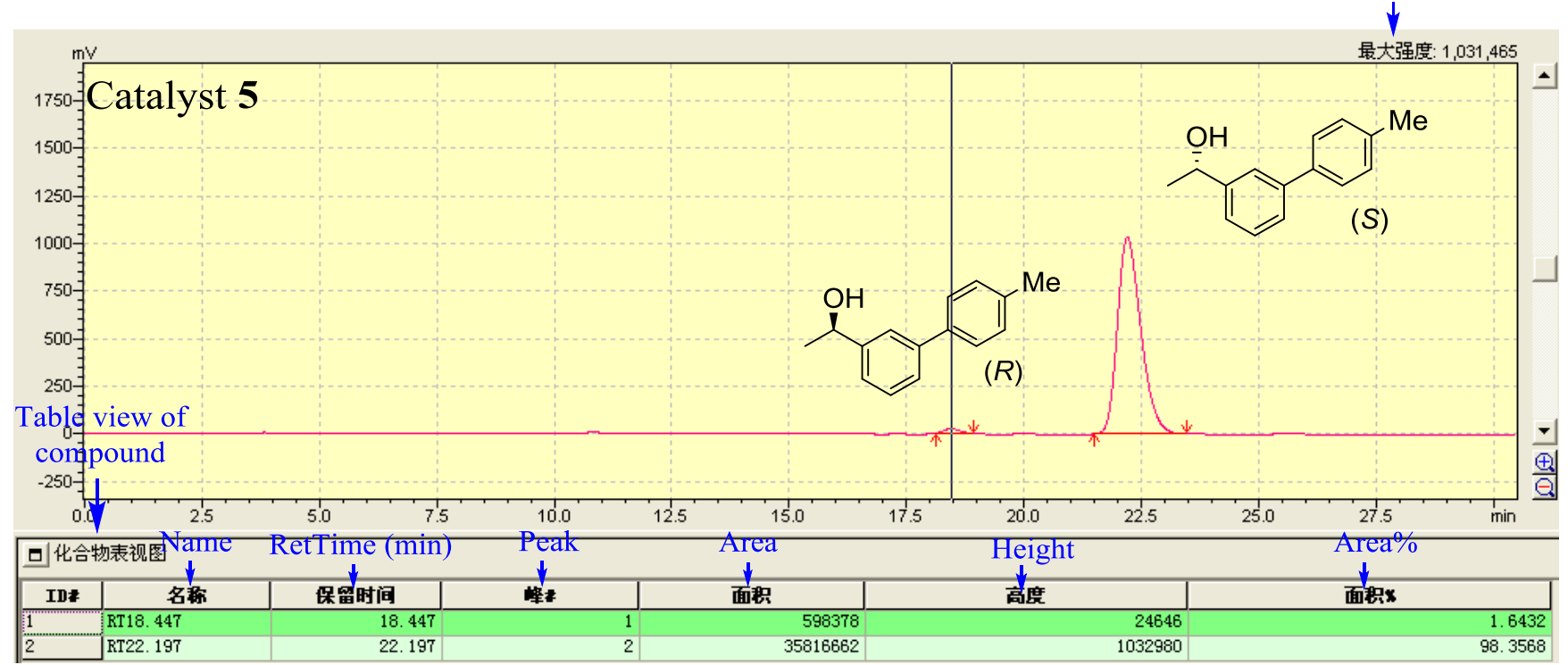

Translation of Chinese into English as follows.

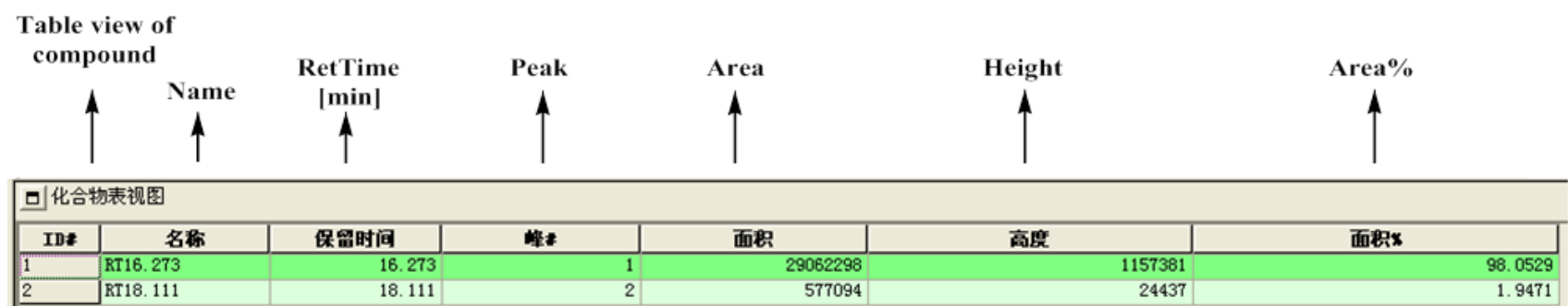


(S)-1-(3-(3-methyl)phenyl)ethanol (9r): (HPLC: Chiracel AD-H, detected at $254 \mathrm{~nm}$, eluent: $\mathrm{n}$-hexane $/ 2-$ propanol $=97 / 3$, flow rate $=1.0 \mathrm{~mL} / \mathrm{min}, 25^{\circ} \mathrm{C}$ ).
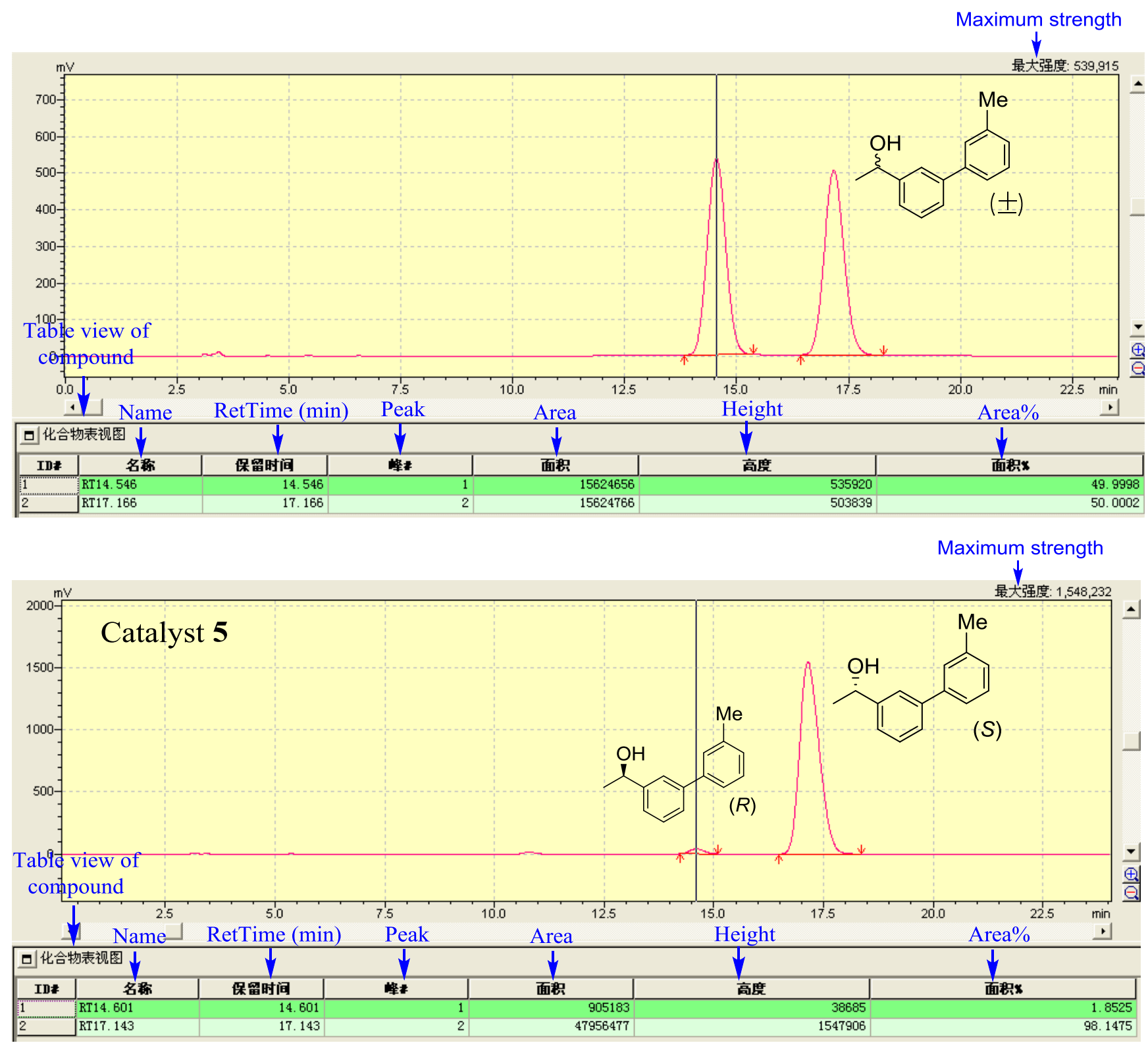

\section{Translation of Chinese into English as follows.}

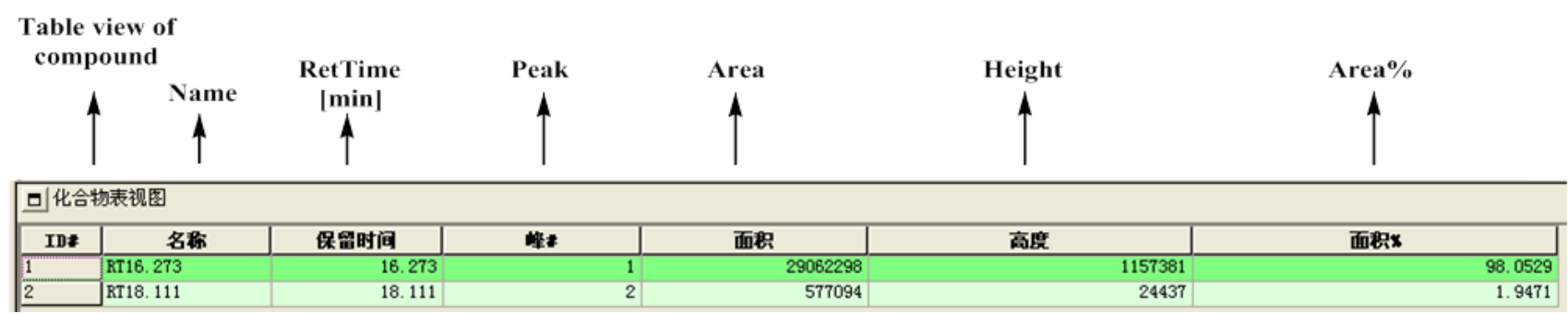


(S)-1-(3-(4-methoxy)phenyl)ethanol (9s): (HPLC: Chiracel AD-H, detected at $254 \mathrm{~nm}$, eluent: $\mathrm{n}$-hexane $/ 2-$ propanol $=97 / 3$, flow rate $=1.0 \mathrm{~mL} / \mathrm{min}, 25^{\circ} \mathrm{C}$ ).
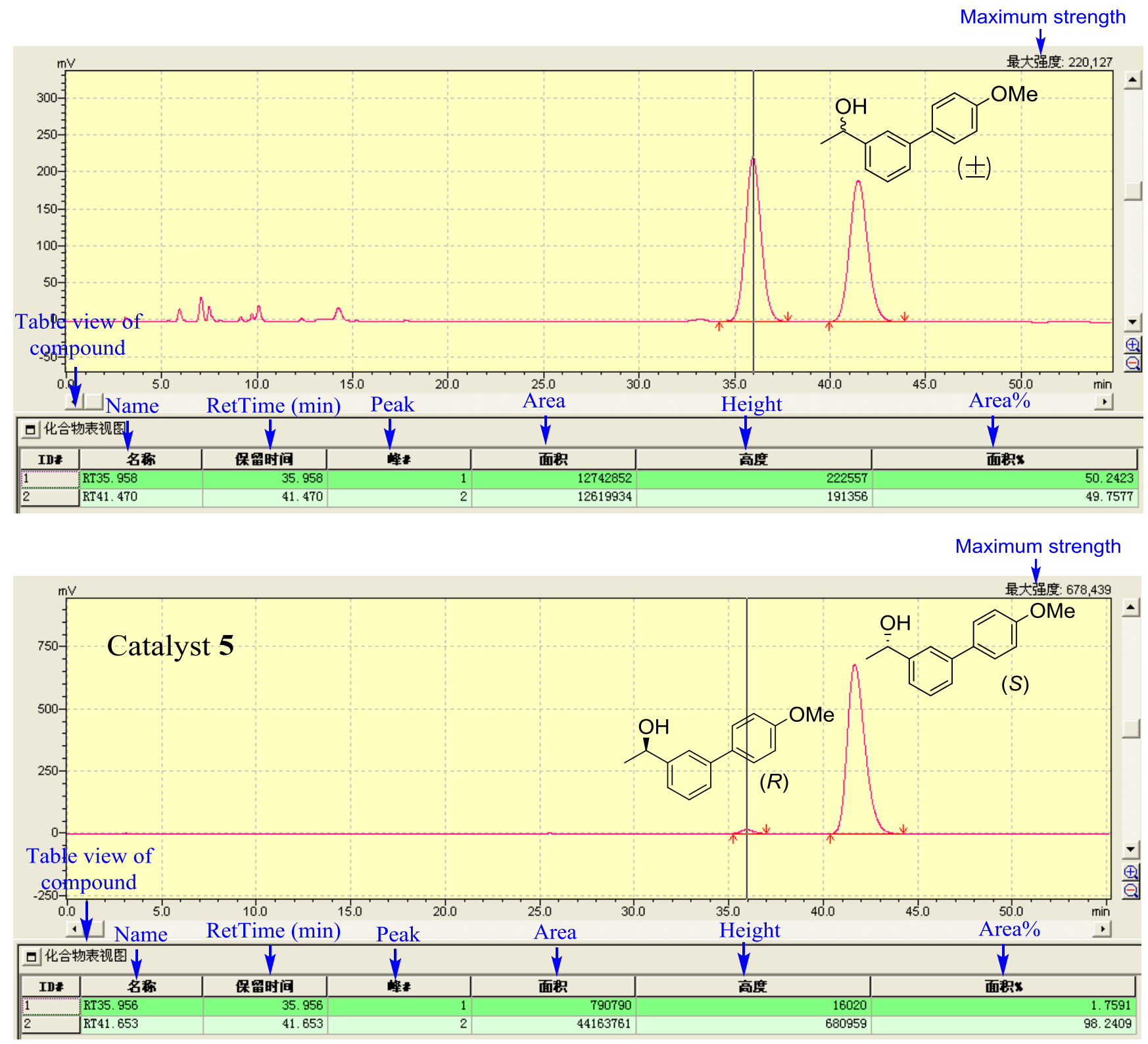

Translation of Chinese into English as follows.

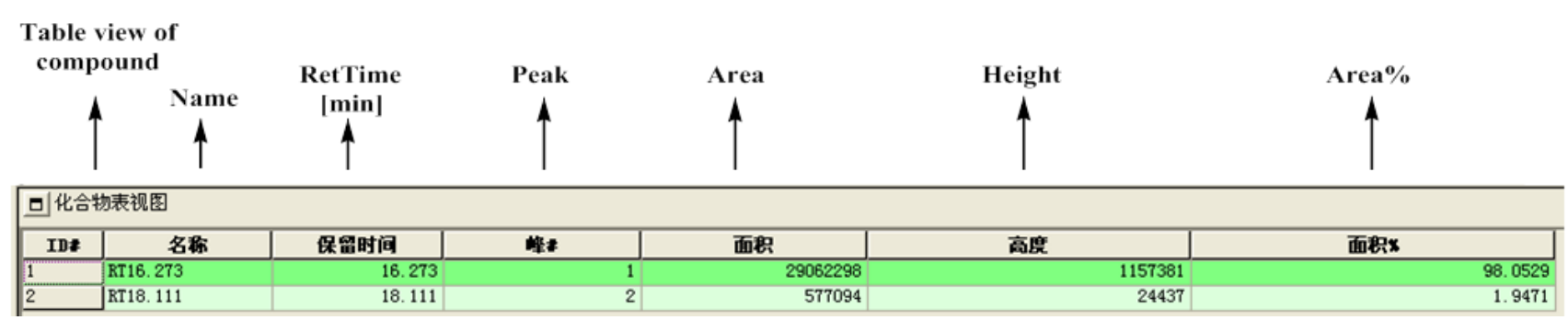


(S)-1-(3-(thiophen-3-yl)phenyl)ethanol (9t): (HPLC: Chiracel AD-H, detected at 254 nm, eluent: nhexane $/ 2$-propanol $=97 / 3$, flow rate $=1.0 \mathrm{~mL} / \mathrm{min}, 25^{\circ} \mathrm{C}$ ).
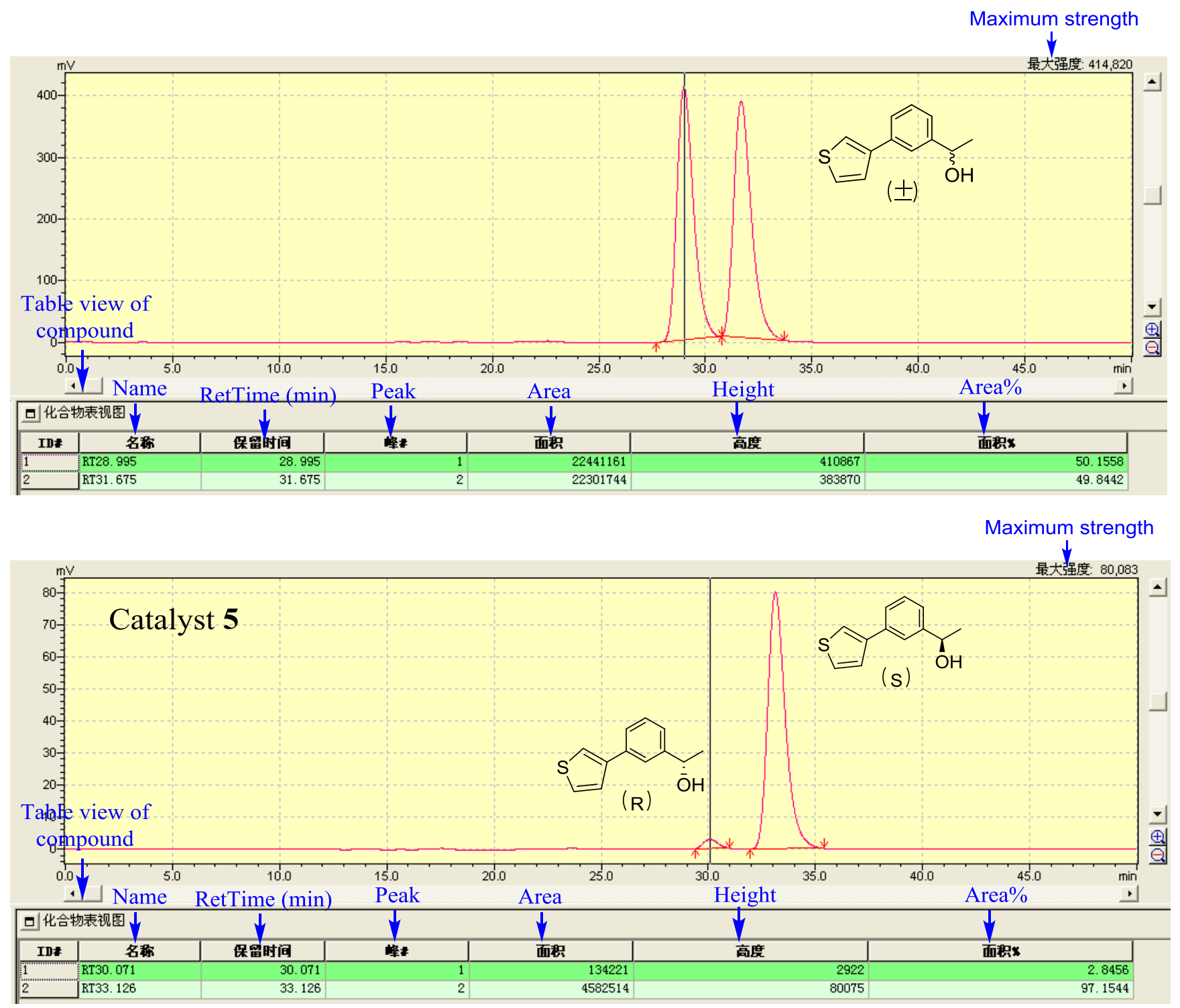

Translation of Chinese into English as follows.

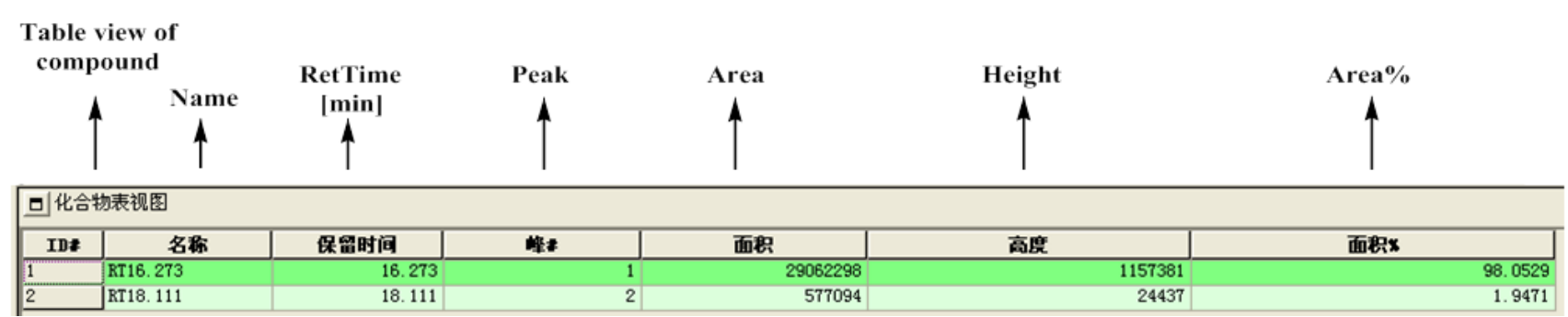


(S)-1-(3-(pyridin-4-yl)phenyl)ethanol (9u): (HPLC: Chiracel OD-H, detected at 254 nm, eluent: nhexane $/ 2$ - propanol $=95 / 05$, flow rate $=0.8 \mathrm{~mL} / \mathrm{min}, 25^{\circ} \mathrm{C}$ ).

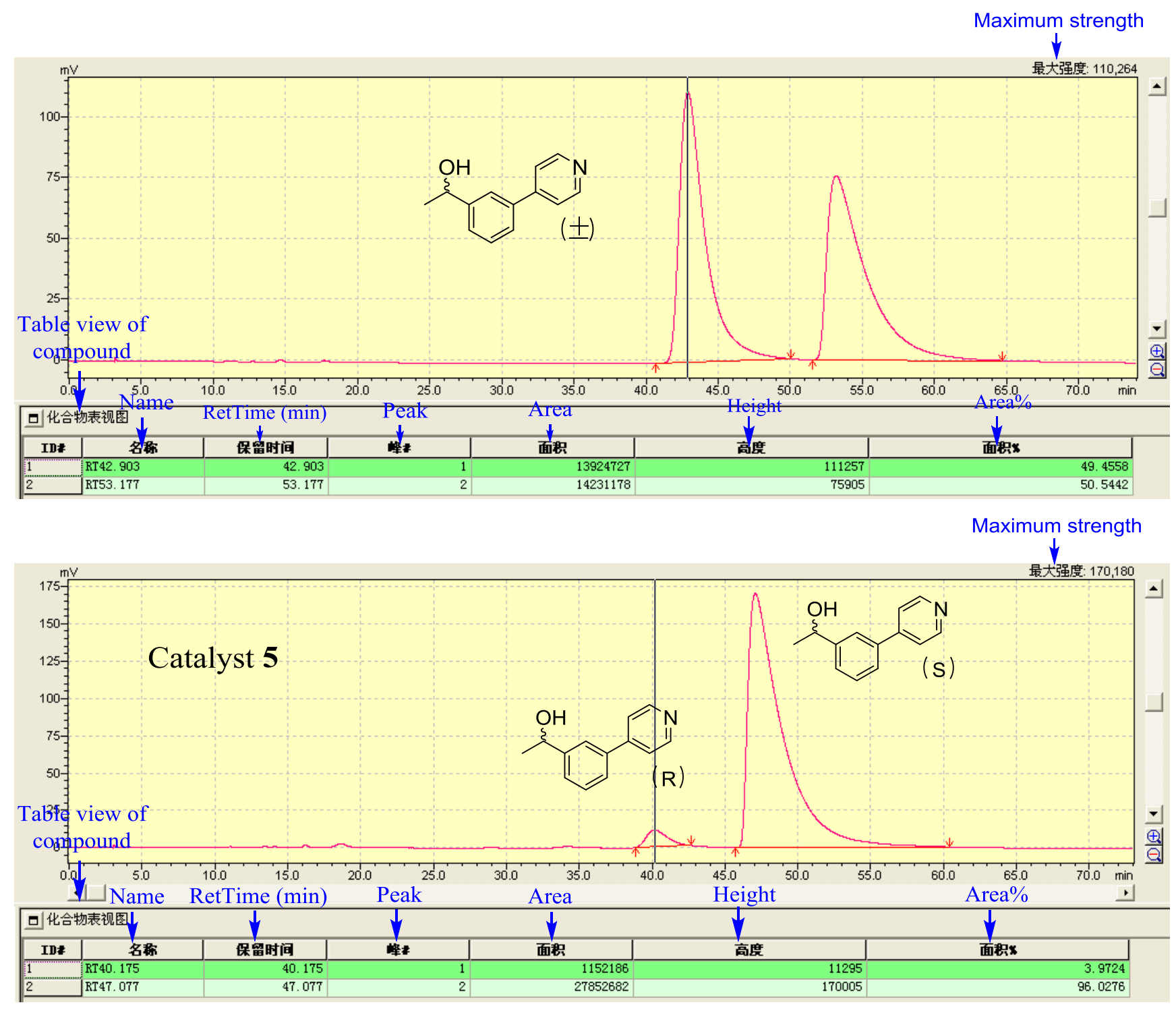

Translation of Chinese into English as follows.

\begin{tabular}{|c|c|c|c|c|c|c|}
\hline \multicolumn{7}{|c|}{$\begin{array}{l}\text { Table view of } \\
\text { compound }\end{array}$} \\
\hline & $\begin{array}{l}\text { ound Name } \\
\text { Name }\end{array}$ & RetTime & Peak & Area & Height & Area \% \\
\hline \multicolumn{7}{|c|}{ 可化合物表视圈 } \\
\hline IDE & 名称 & 保留时闻 & 製 & 面知 & 高度 & 面积x \\
\hline 1 & RT16.273 & 16.273 & 1 & 29062298 & 1157381 & 98.0529 \\
\hline 2 & RT18.111 & 18.111 & 2 & 577094 & 24437 & 1. 9471 \\
\hline
\end{tabular}


(S)-1-phenyl-3-(phenylamino)propan-1-ol (12a): HPLC : Chiracel OD-H, detected at $254 \mathrm{~nm}$, elute: $\mathrm{n}$-hexane/i-PrOH $=90 / 10$, flow rate: $1.0 \mathrm{~mL} / \mathrm{min}, 25^{\circ} \mathrm{C}$ ).
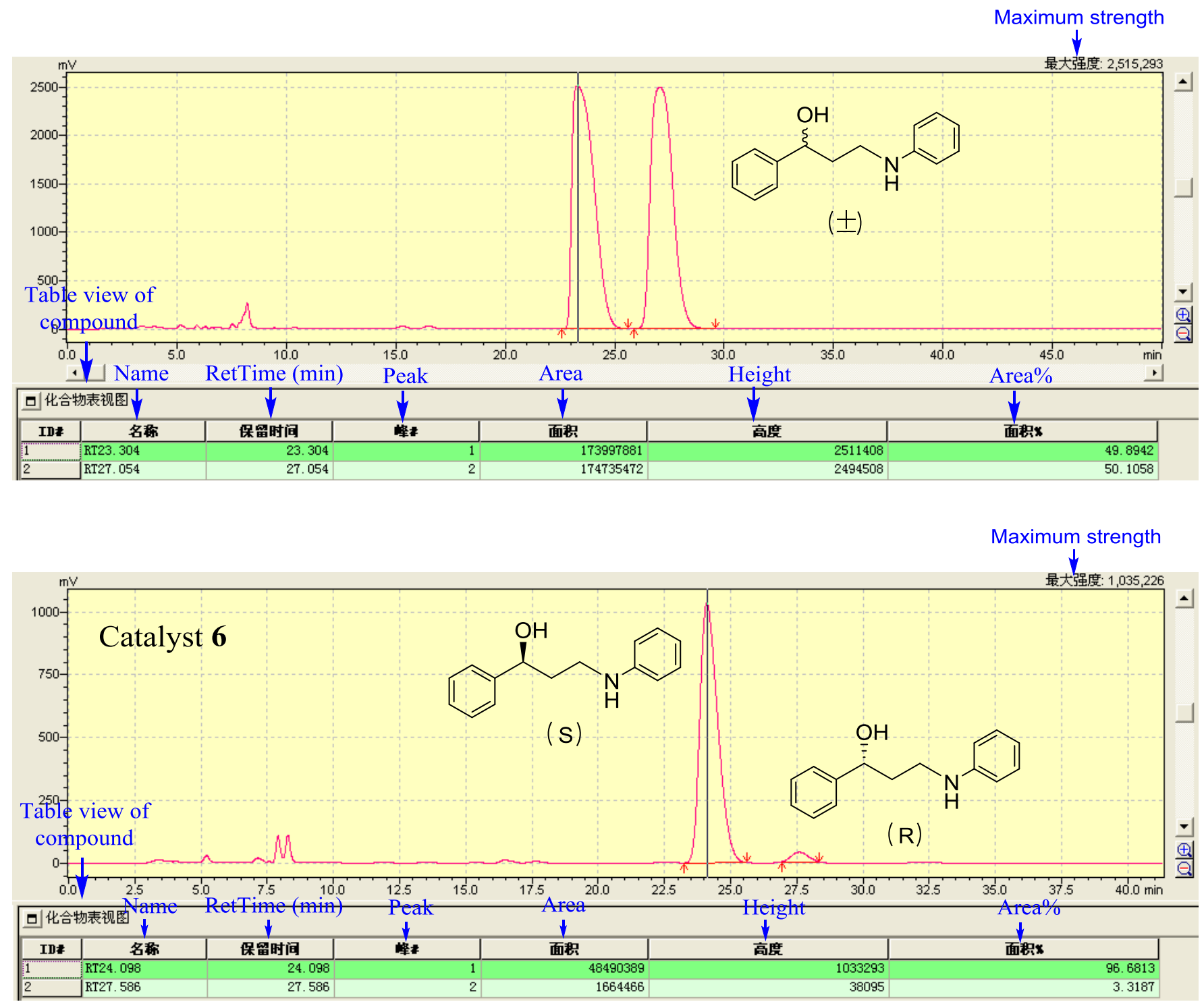

\section{Translation of Chinese into English as follows.}

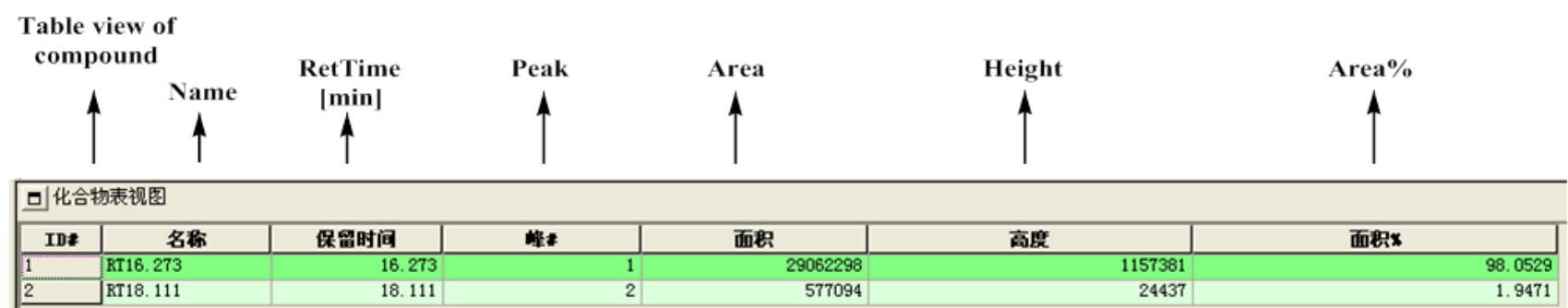


(S)-1-(4-fluorophenyl)-3-(phenylamino)propan-1-ol (12b): HPLC : Chiracel OD-H, detected at $254 \mathrm{~nm}$, elute: $\mathrm{n}$-hexane/i-PrOH $=90 / 10$, flow rate: $1.0 \mathrm{~mL} / \mathrm{min}, 25^{\circ} \mathrm{C}$ ).
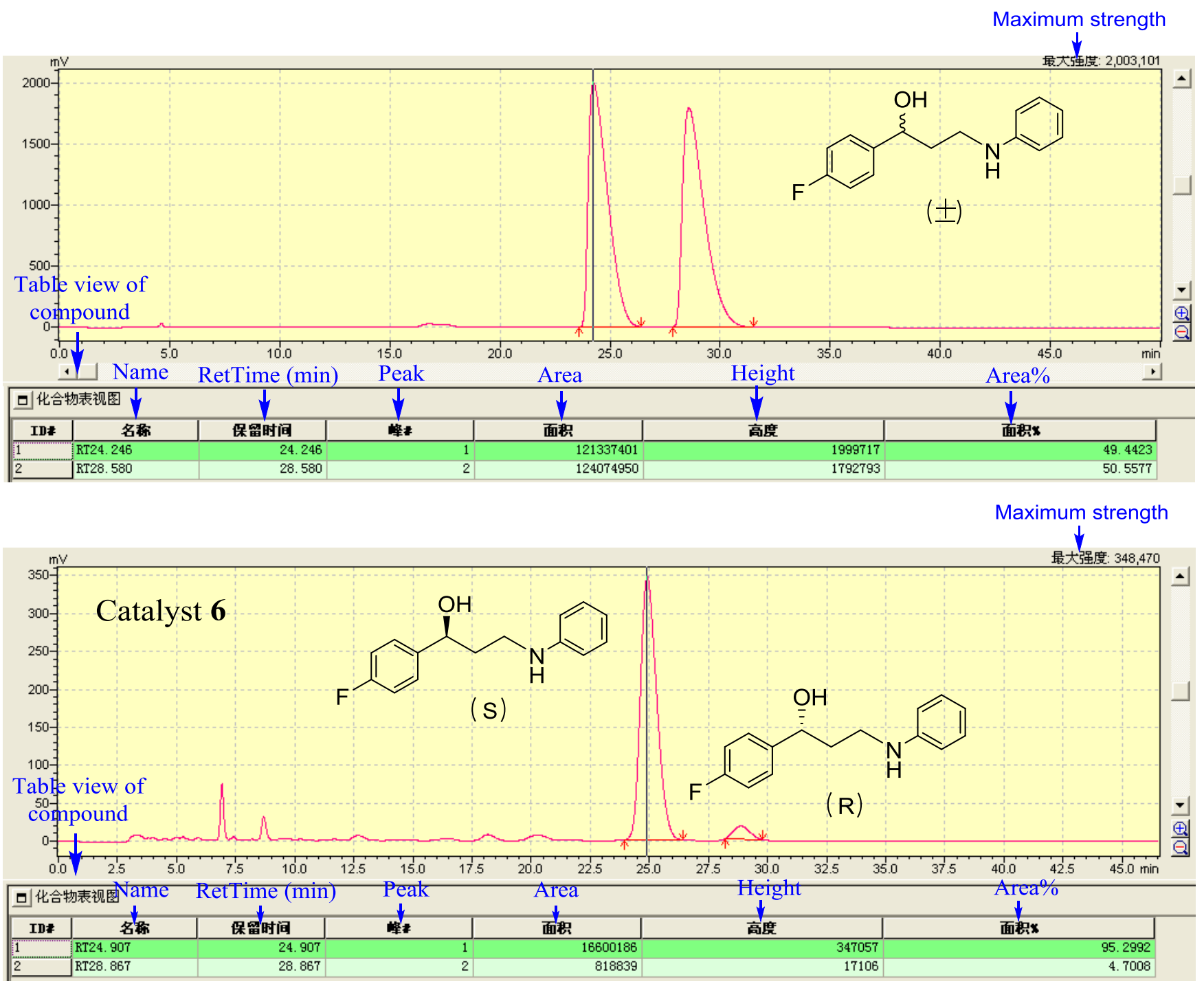

Translation of Chinese into English as follows.

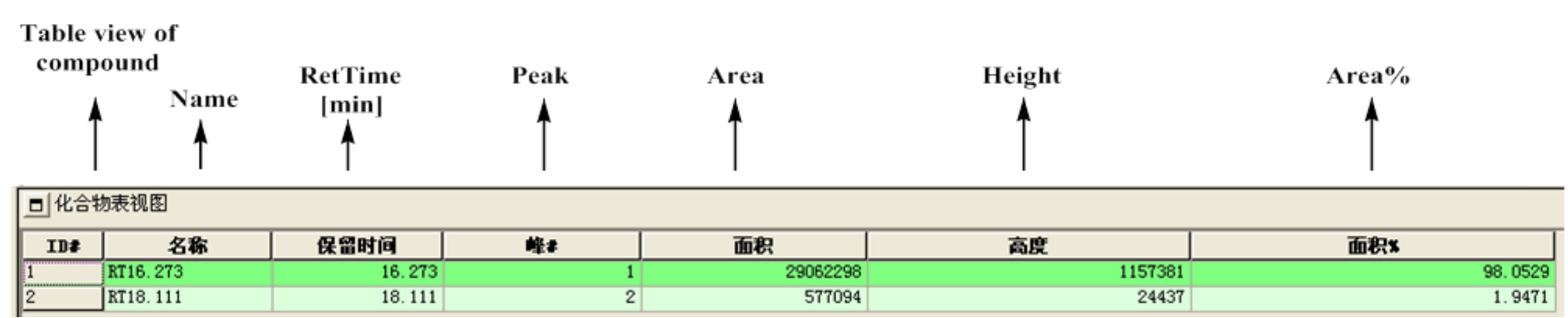


(S)-1-(4-chlorophenyl)-3-(phenylamino)propan-1-ol (12c): HPLC : Chiracel OD-H, detected at $254 \mathrm{~nm}$, elute: $\mathrm{n}$-hexane/i-PrOH $=90 / 10$, flow rate: $1.0 \mathrm{~mL} / \mathrm{min}, 25^{\circ} \mathrm{C}$ ).
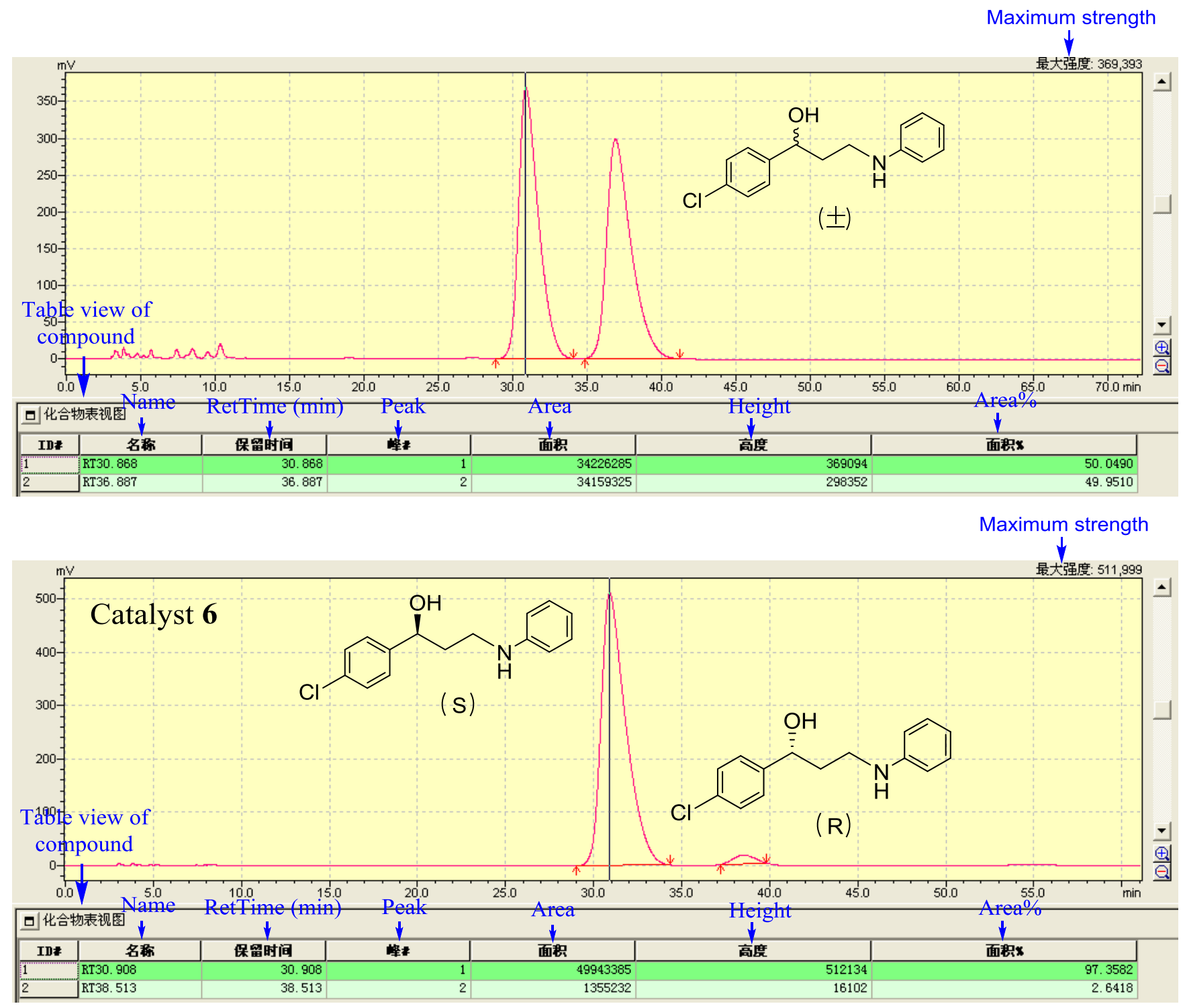

\section{Translation of Chinese into English as follows.}

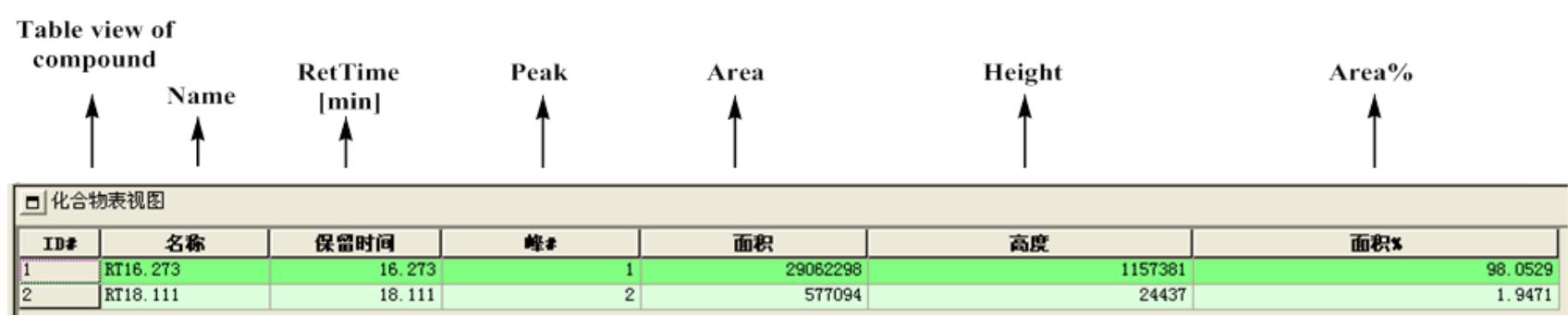


(S)-1-(4-bromophenyl)-3-(phenylamino)propan-1-ol (12d): HPLC : Chiracel AS-H, detected at $254 \mathrm{~nm}$, elute: $\mathrm{n}$-hexane/i-PrOH $=90 / 10$, flow rate: $1.0 \mathrm{~mL} / \mathrm{min}, 25^{\circ} \mathrm{C}$ ).
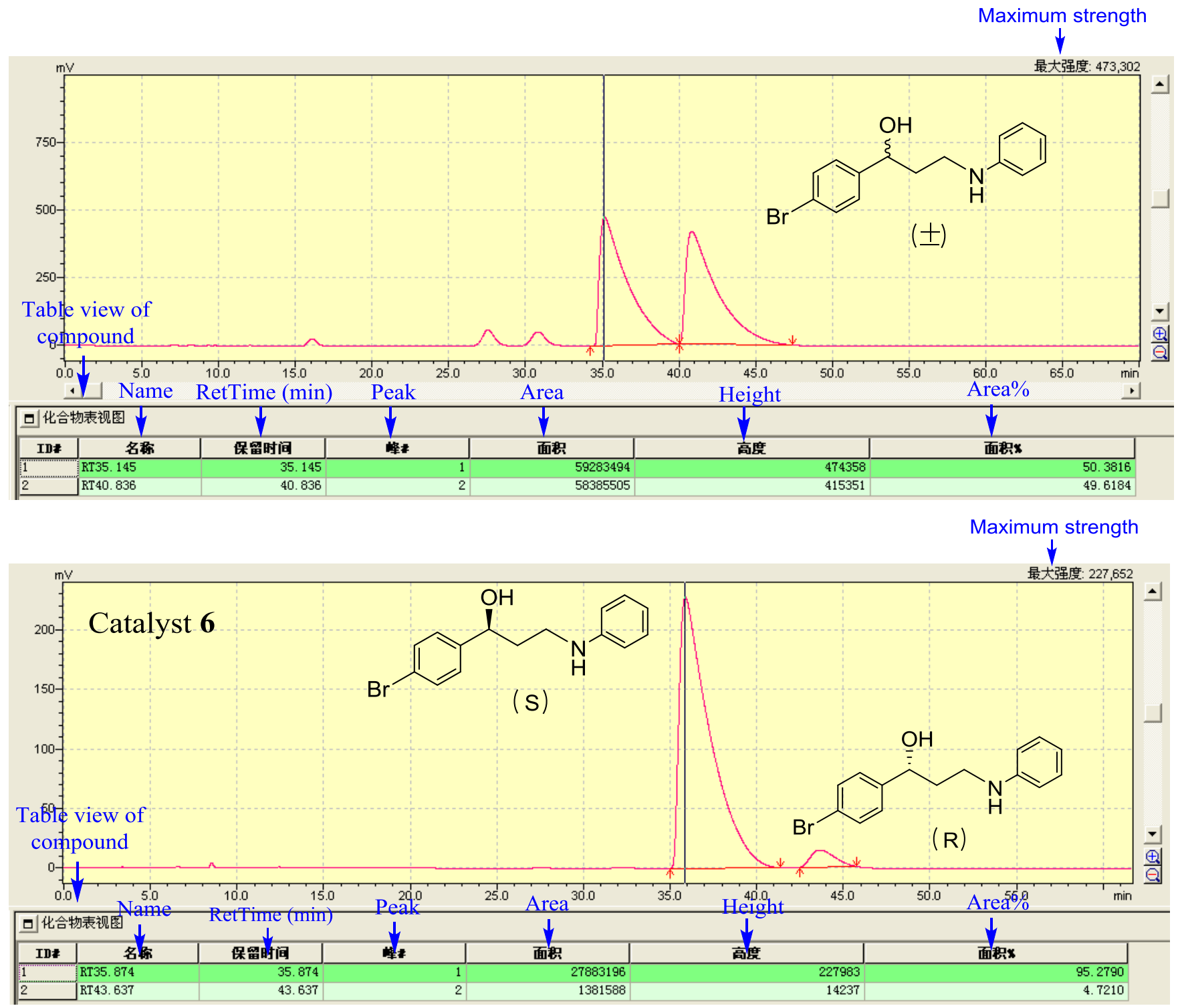

Translation of Chinese into English as follows.

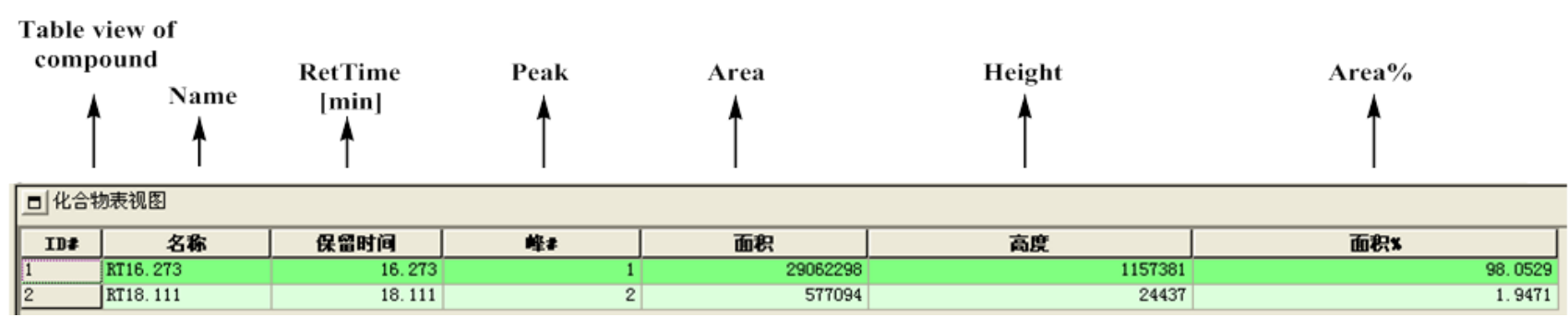


(S)-1-(4-iodophenyl)-3-(phenylamino)propan-1-ol (12e): HPLC : Chiracel AS-H, detected at 254 nm, elute: $\mathrm{n}$-hexane/i-PrOH $=90 / 10$, flow rate: $1.0 \mathrm{~mL} / \mathrm{min}, 25^{\circ} \mathrm{C}$ ).
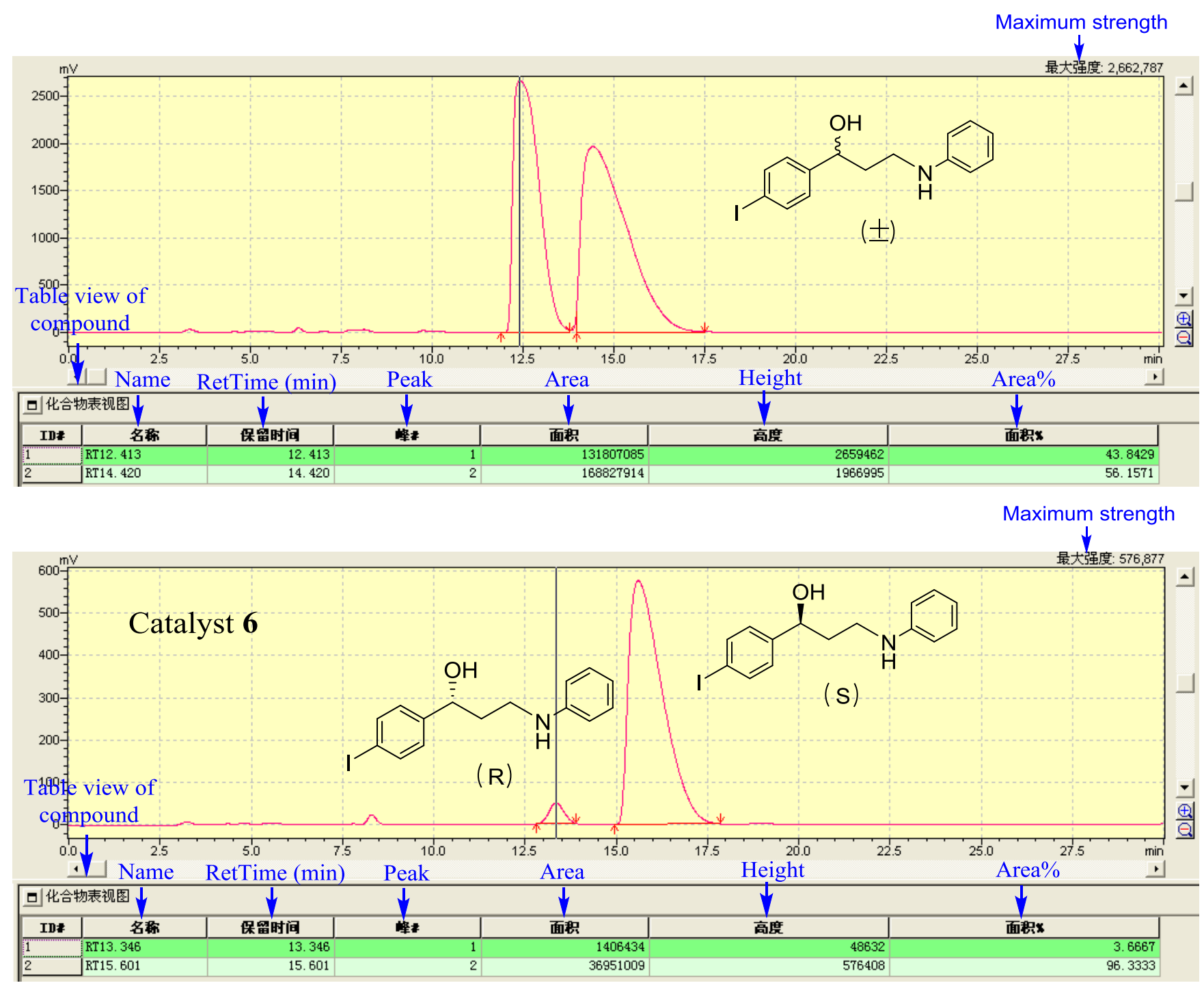

Translation of Chinese into English as follows.

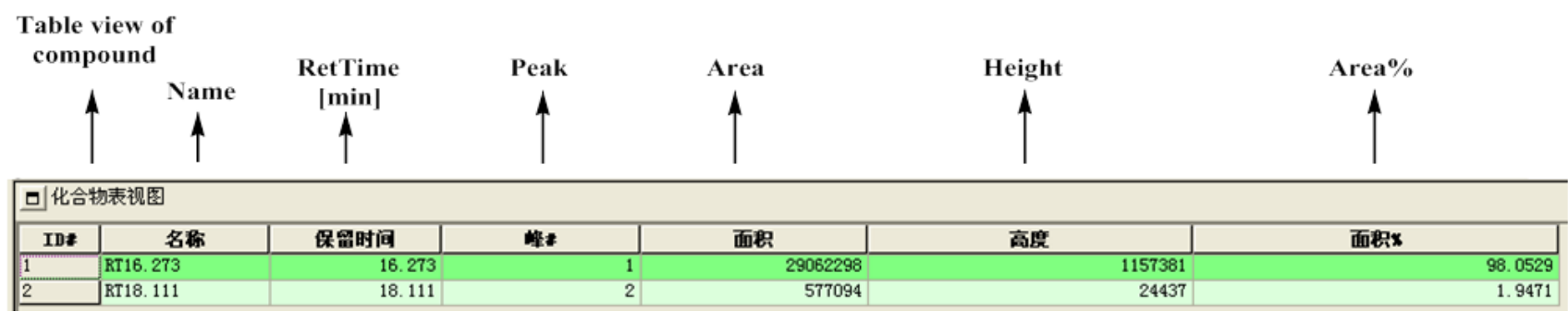


(S)-4-(1-hydroxy-3-(phenylamino)propyl)benzonitrile (12f): HPLC : Chiracel AS-H, detected at $254 \mathrm{~nm}$, elute: $\mathrm{n}$-hexane/i-PrOH $=90 / 10$, flow rate: $1.0 \mathrm{~mL} / \mathrm{min}, 25^{\circ} \mathrm{C}$ ).
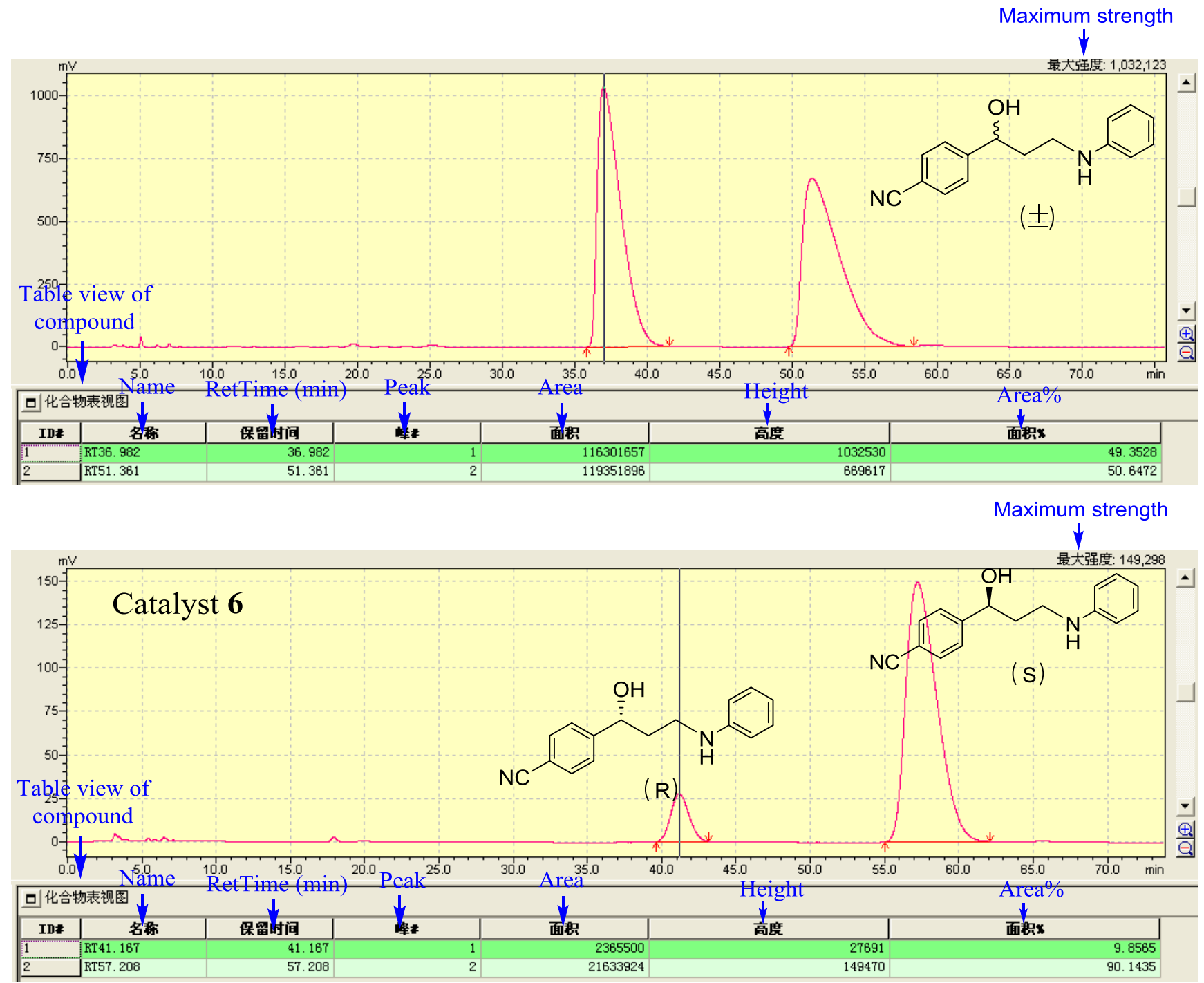

Translation of Chinese into English as follows.

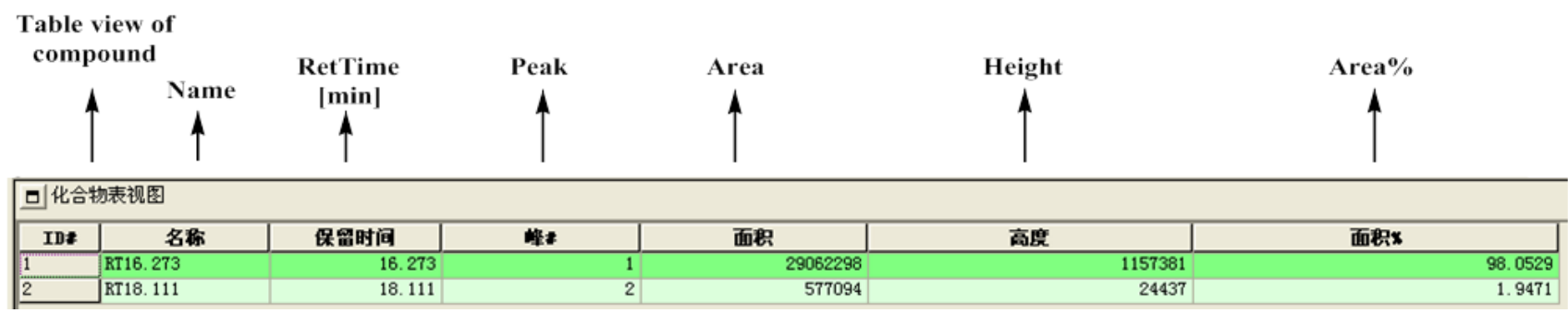


(S)-3-(phenylamino)-1-(p-tolyl)propan-1-ol (12g): HPLC : Chiracel OD-H, detected at $254 \mathrm{~nm}$, elute: $\mathrm{n}$-hexane/i-PrOH $=90 / 10$, flow rate: $1.0 \mathrm{~mL} / \mathrm{min}, 25^{\circ} \mathrm{C}$ ).

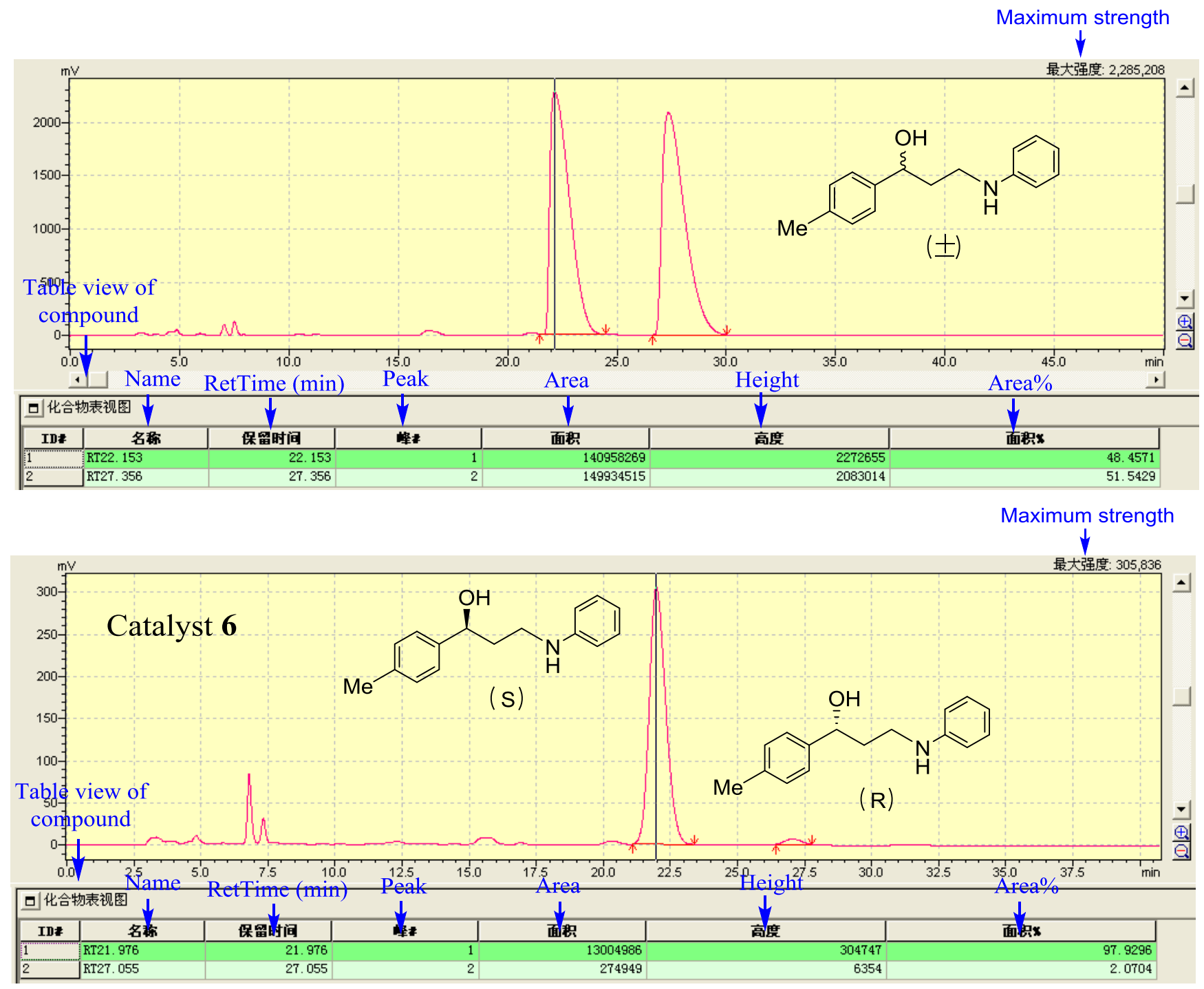

Translation of Chinese into English as follows.

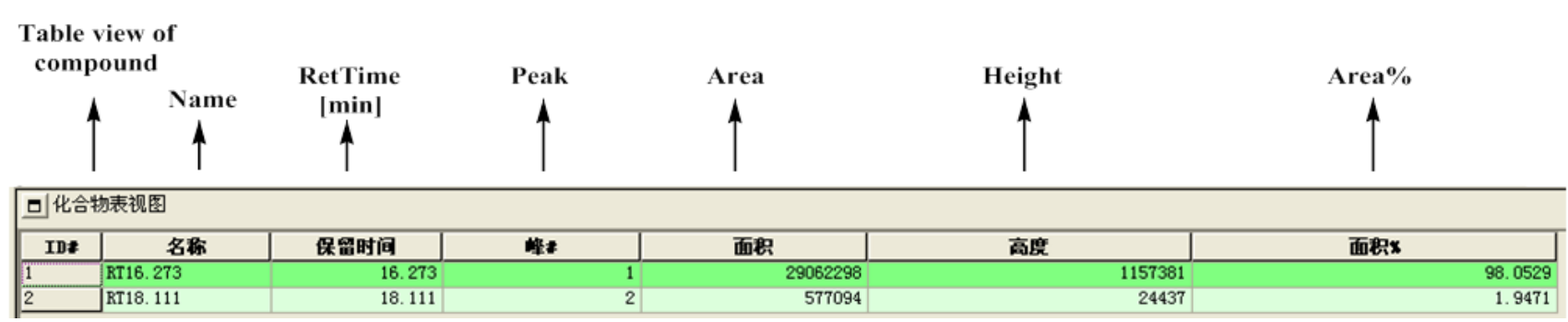


(S)-1-(4-methoxyphenyl)-3-(phenylamino)propan-1-ol (12h): HPLC : Chiracel OD-H, detected at $254 \mathrm{~nm}$, elute: $\mathrm{n}$-hexane/i-PrOH $=90 / 10$, flow rate: $1.0 \mathrm{~mL} / \mathrm{min}, 25^{\circ} \mathrm{C}$ ).
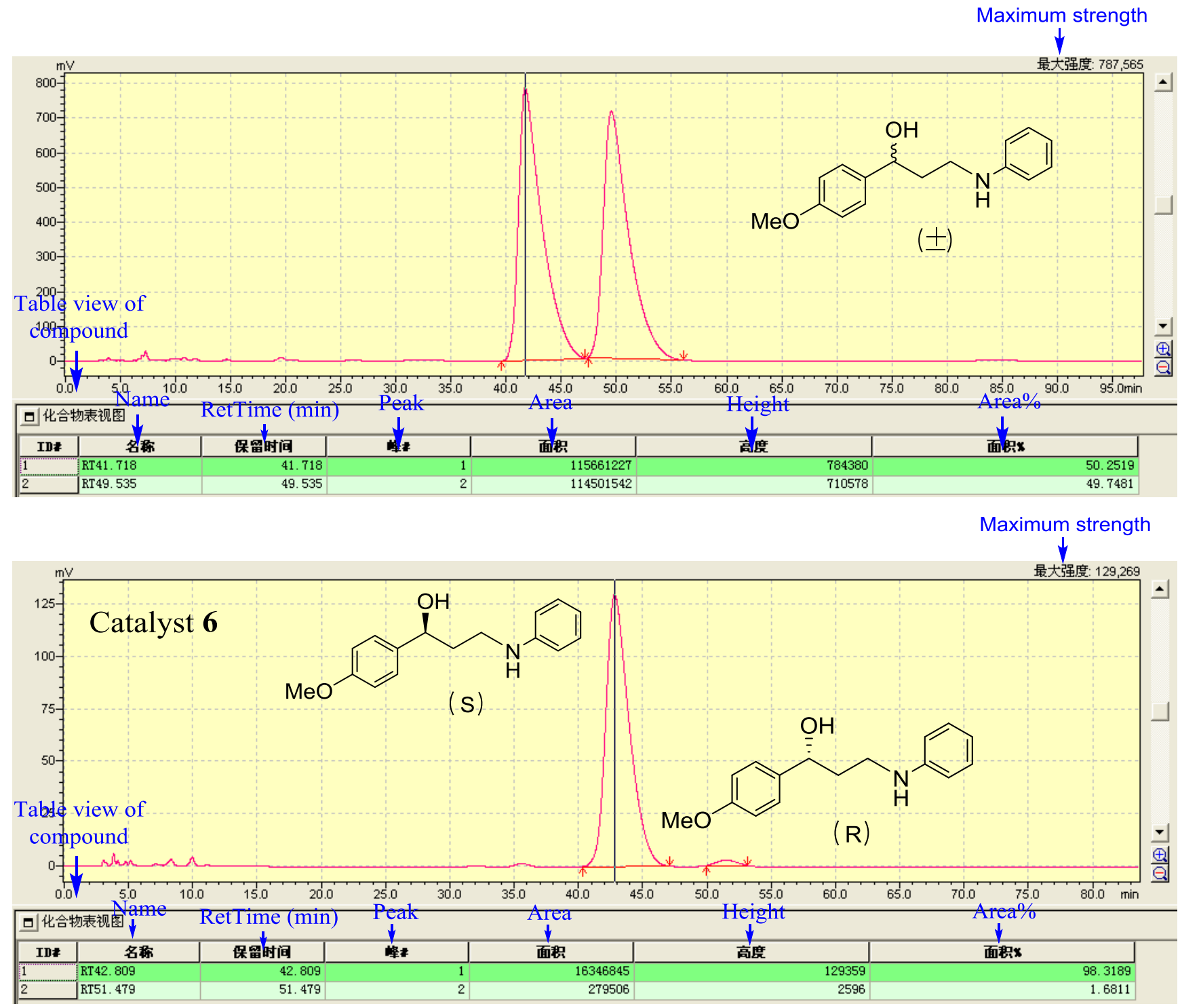

$\underline{\text { Translation of Chinese into English as follows. }}$

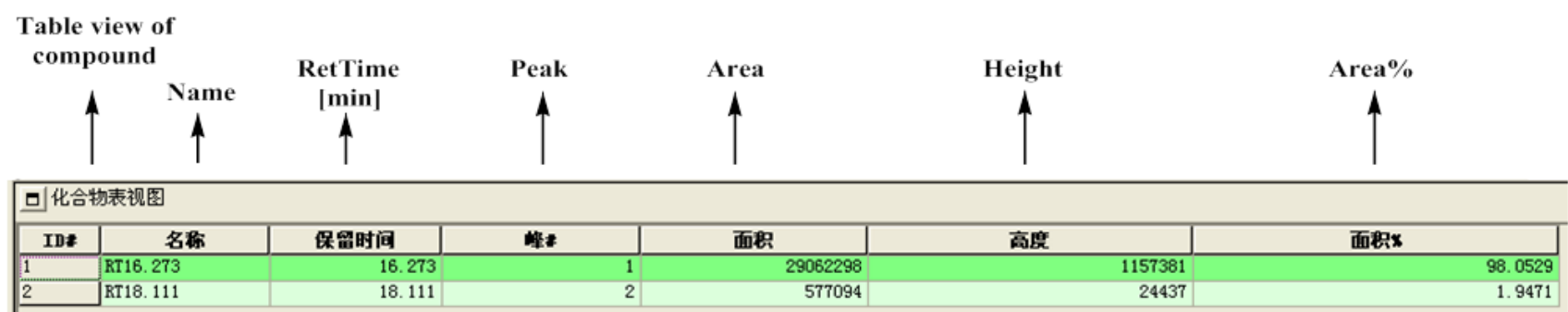


(S)-3-((4-chlorophenyl)amino)-1-phenylpropan-1-ol (12i): HPLC : Chiracel OD-H, detected at $254 \mathrm{~nm}$, elute: $\mathrm{n}$-hexane/i-PrOH = 90/10, flow rate: $1.0 \mathrm{~mL} / \mathrm{min}, 25^{\circ} \mathrm{C}$ ).
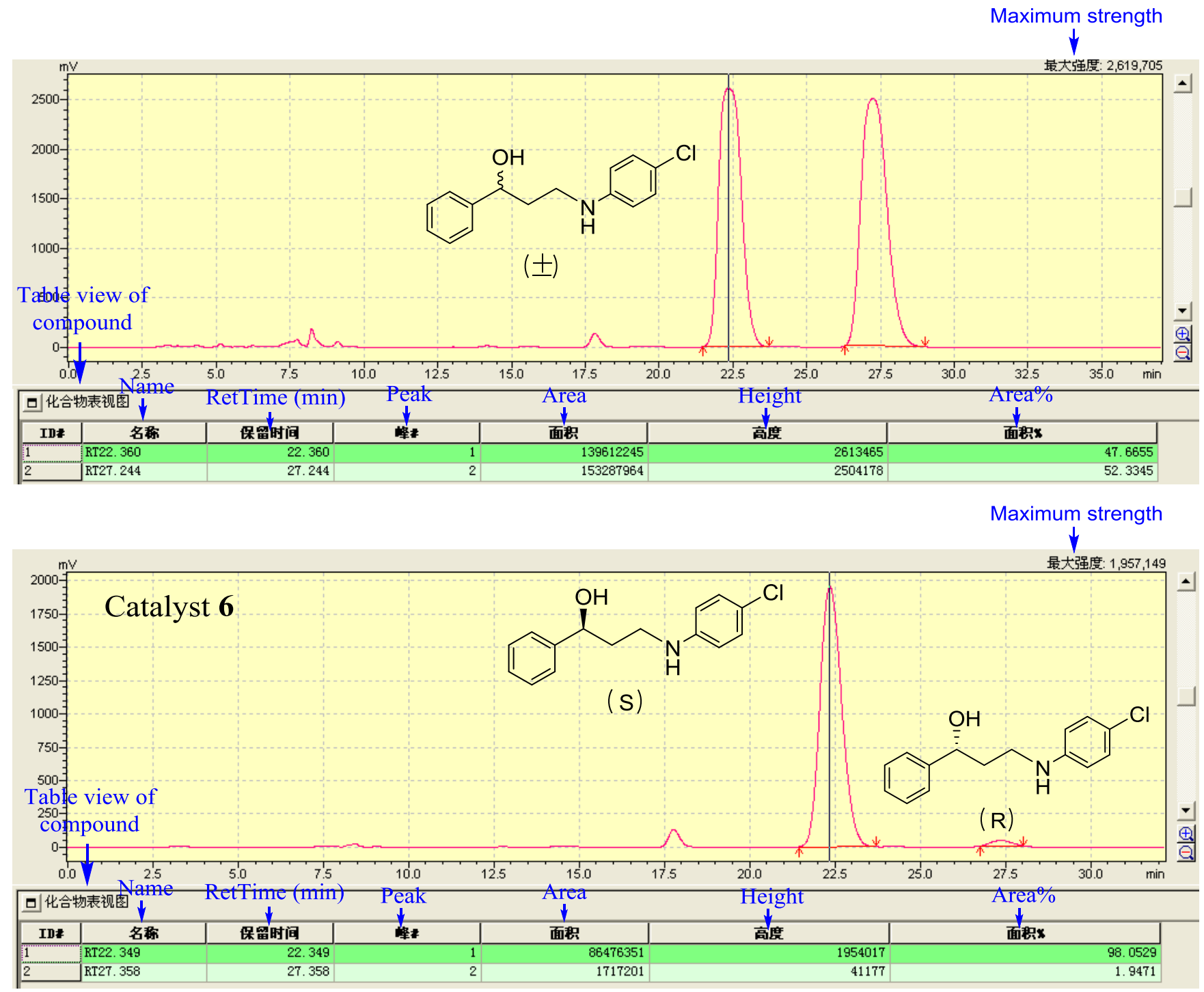

Translation of Chinese into English as follows.

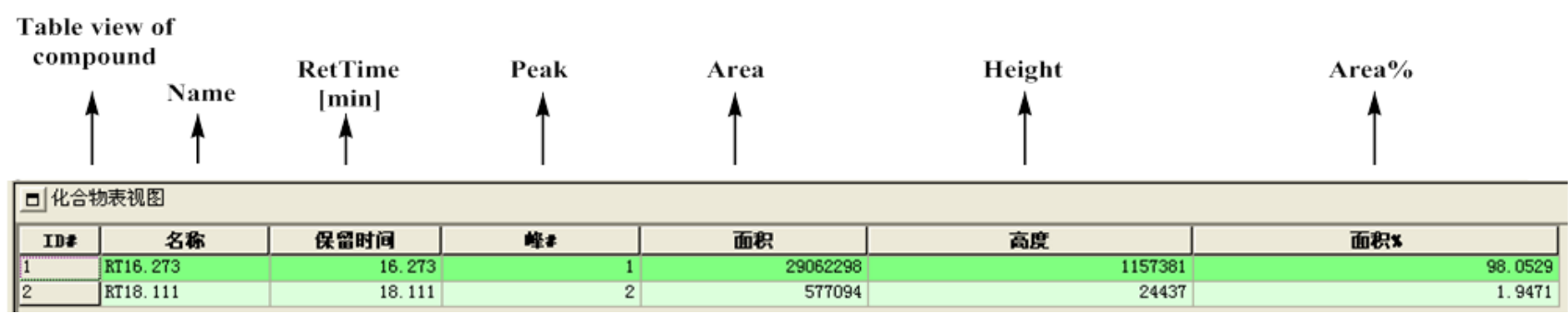


(S)-3-((3-chlorophenyl)amino)-1-phenylpropan-1-ol (12j): HPLC : Chiracel OD-H, detected at $254 \mathrm{~nm}$, elute: $\mathrm{n}$-hexane/i-PrOH $=90 / 10$, flow rate: $1.0 \mathrm{~mL} / \mathrm{min}, 25^{\circ} \mathrm{C}$ ).

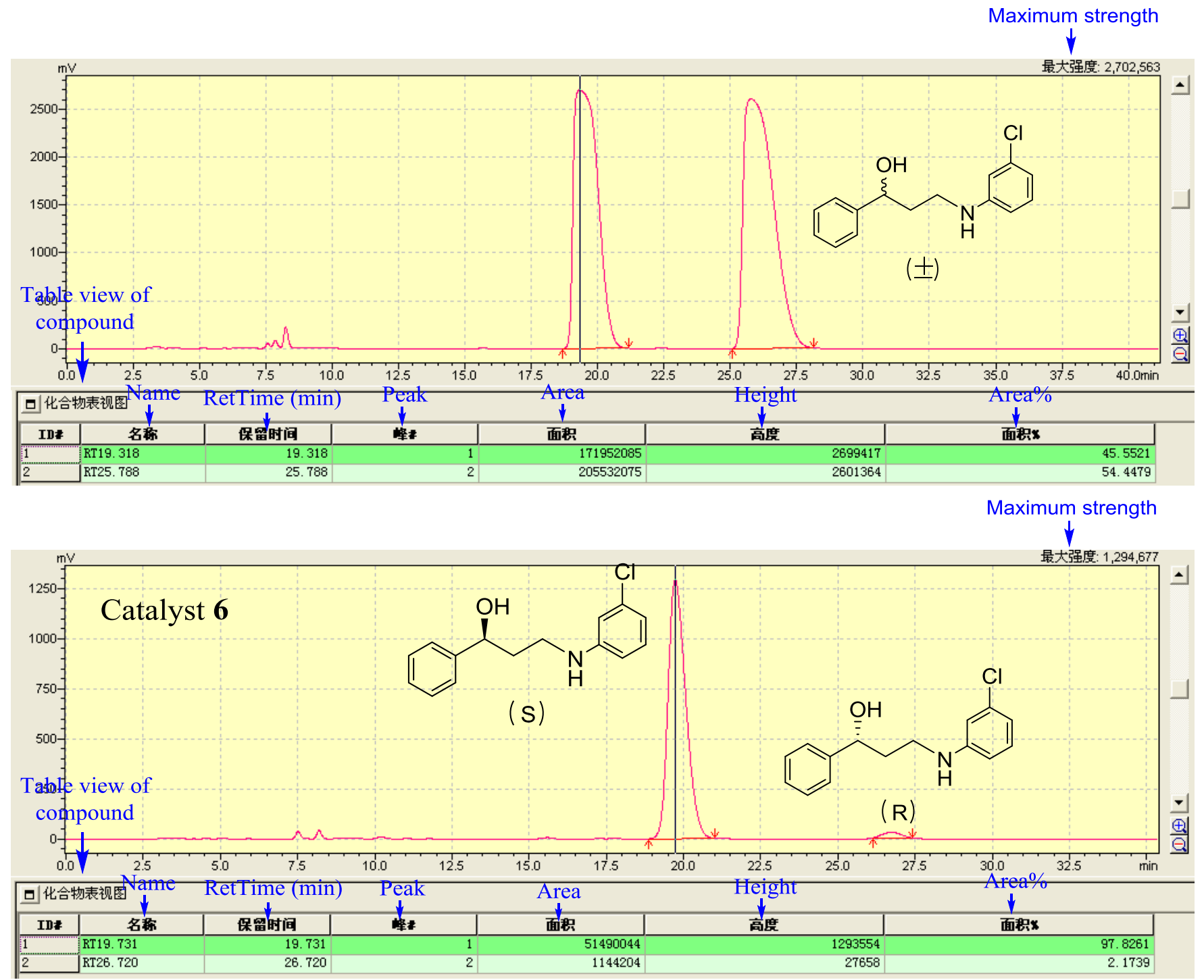

Translation of Chinese into English as follows.

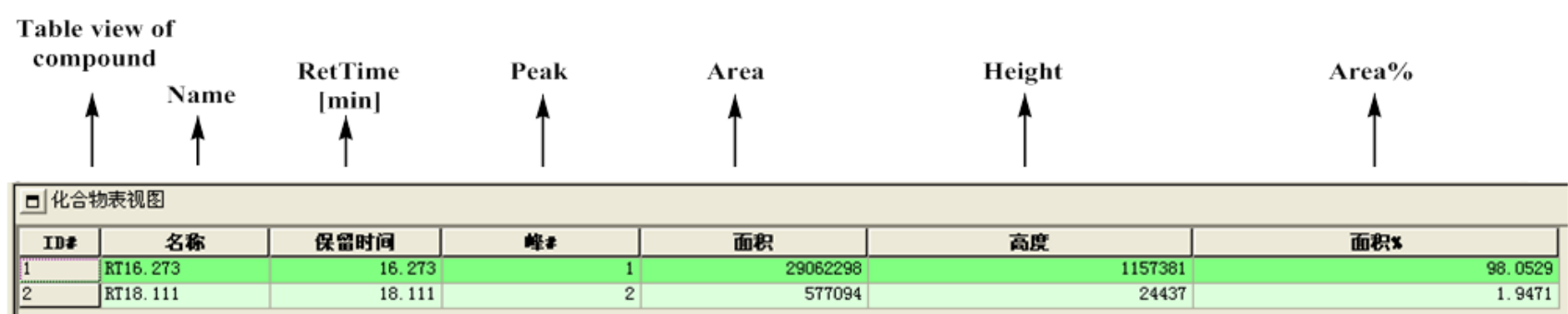


(S)-3-((4-bromophenyl)amino)-1-phenylpropan-1-ol (12k): HPLC : Chiracel OD-H, detected at $254 \mathrm{~nm}$, elute: $\mathrm{n}$-hexane/i-PrOH $=90 / 10$, flow rate: $1.0 \mathrm{~mL} / \mathrm{min}, 25^{\circ} \mathrm{C}$ ).

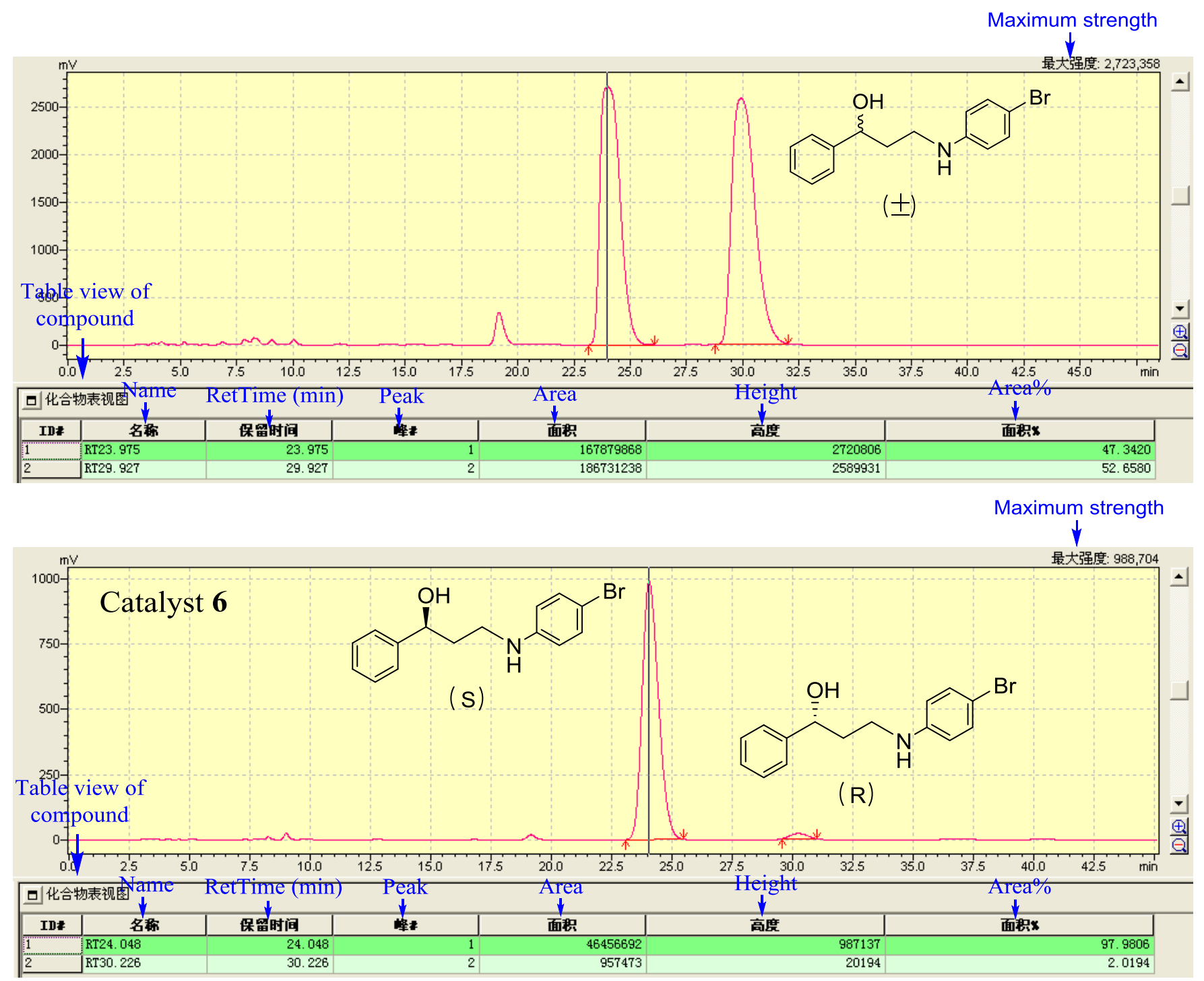

Translation of Chinese into English as follows.

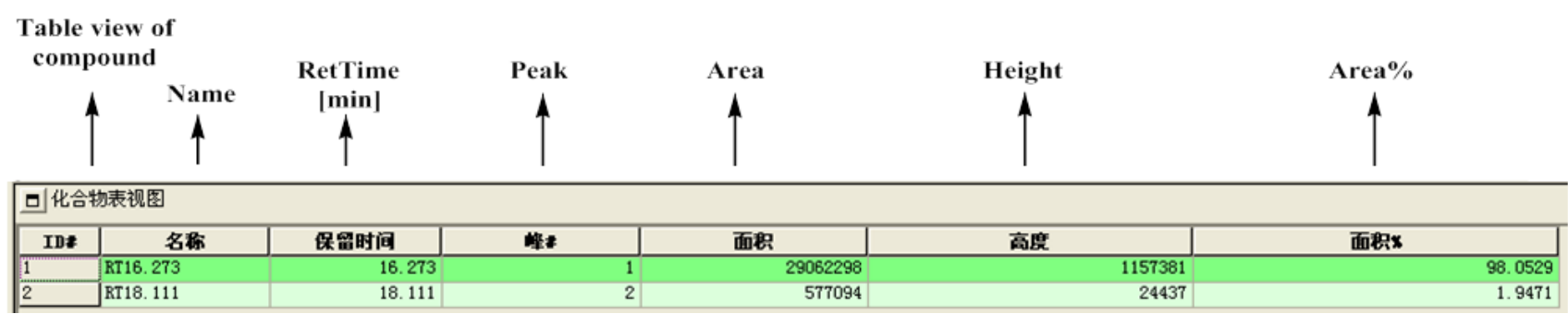


(S)-1-phenyl-3-(p-tolylamino)propan-1-ol (12I): HPLC : Chiracel OD-H, detected at $254 \mathrm{~nm}$, elute: $\mathrm{n}$-hexane/i-PrOH $=90 / 10$, flow rate: $1.0 \mathrm{~mL} / \mathrm{min}, 25^{\circ} \mathrm{C}$ ).

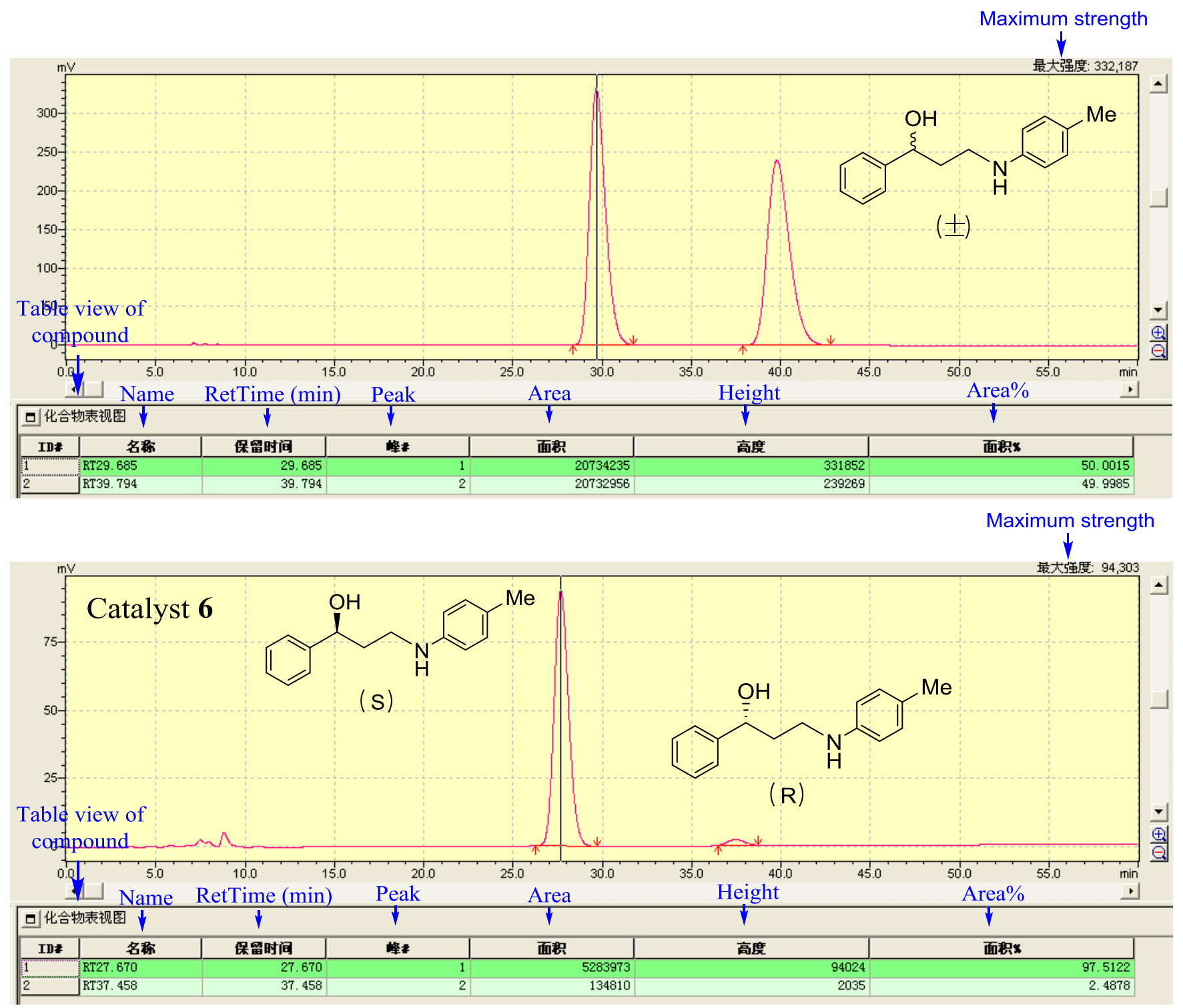

Translation of Chinese into English as follows.

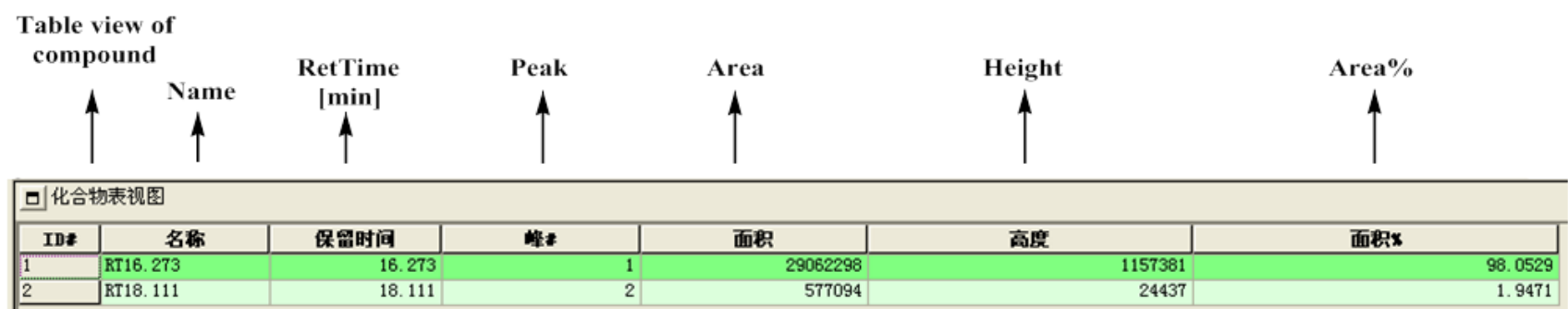


(S)-3-((4-methoxyphenyl)amino)-1-phenylpropan-1-ol (12m): HPLC : Chiracel OD-H, detected at $254 \mathrm{~nm}$, elute: $\mathrm{n}$-hexane/i-PrOH = 90/10, flow rate: $1.0 \mathrm{~mL} / \mathrm{min}, 25^{\circ} \mathrm{C}$ ).

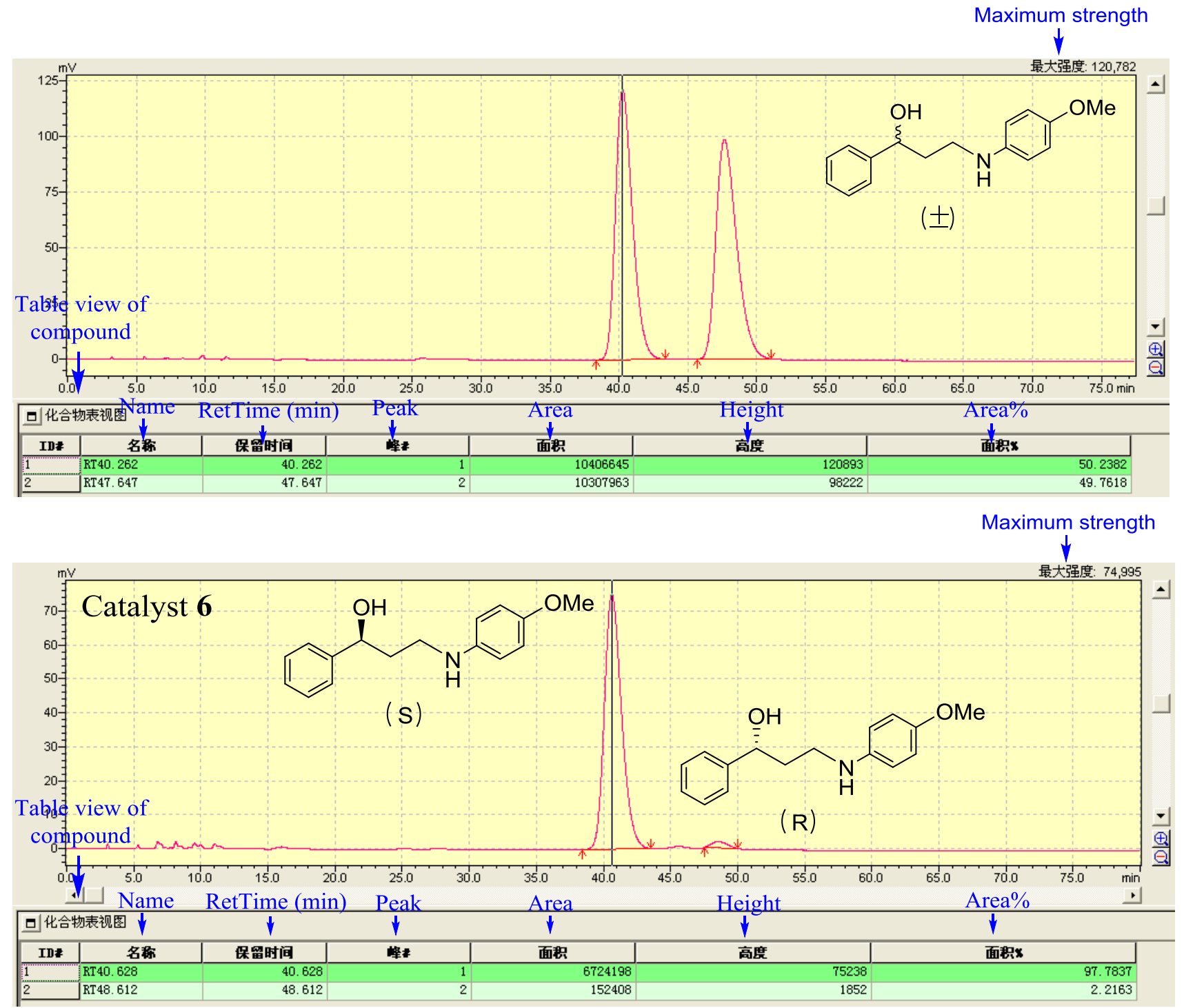

Translation of Chinese into English as follows.

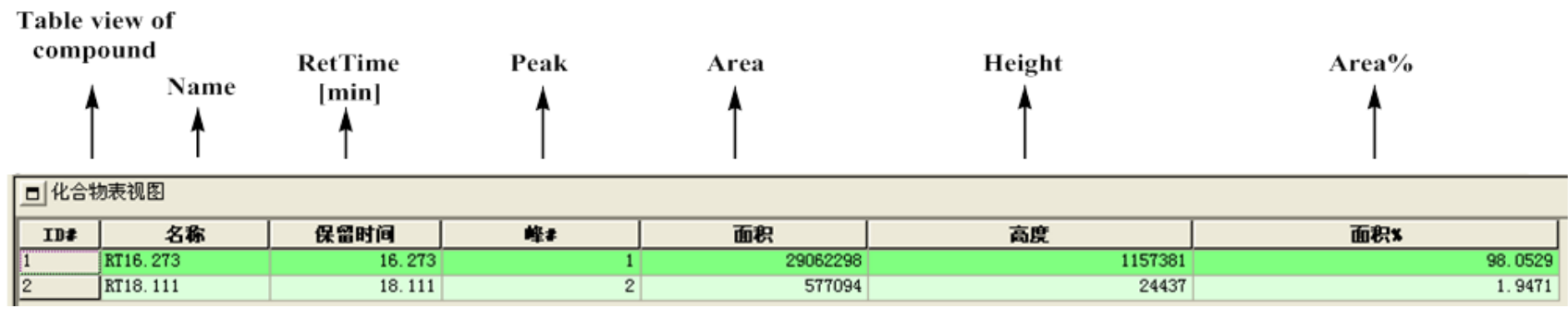

Figure S12. HPLC analyses for chiral products. 
Table S2. Reusability of catalyst 5 for the Suzuki cross-coupling/ATH of cascade reactions of 4-chloroacetophenone and phenylboronic acid. (Reaction conditions: Catalyst 5 (130.70 mg, $20.0 \mu \mathrm{mol}$ of $\mathrm{Ru}$ and $15.80 \mu \mathrm{mol}$ of $\mathrm{Pd}$, based on ICP analysis $), \mathrm{Cs}_{2} \mathrm{CO}_{3}(1.20 \mathrm{mmol}), \mathrm{HCO}_{2} \mathrm{Na}(10.0 \mathrm{mmol})$, iodoacetophenones $(1.00 \mathrm{mmol})$ and arylboronic acids $(1.20 \mathrm{mmol})$, and $20.0 \mathrm{~mL}$ of $\mathrm{H}_{2} \mathrm{O} / \mathrm{PrOH}(\mathrm{v} / \mathrm{v}=$ 1:1) were added sequentially to a $50.0 \mathrm{~mL}$ round-bottom flask. The mixture was then stirred at $60{ }^{\circ} \mathrm{C}$ for the first 3 hours followed by at $15{ }^{\circ} \mathrm{C}$ for $7 \mathrm{~h}$. The yields are confirmed by ${ }^{1} \mathrm{H}-\mathrm{NMR}$ and the $e e$ values are determined by chiral HPLC analysis after purification by flash-column chromatography).

\begin{tabular}{lllllllll}
\hline Entry & $\mathbf{1}$ & $\mathbf{2}$ & $\mathbf{3}$ & $\mathbf{4}$ & $\mathbf{5}$ & $\mathbf{6}$ & $\mathbf{7}$ & $\mathbf{8}$ \\
\hline Yield(\%) & 96 & 96 & 95 & 95 & 94 & 93 & 86 & 83 \\
ee $(\%)$ & 96 & 96 & 96 & 95 & 95 & 95 & 94 & 94 \\
\hline
\end{tabular}

\section{Recycling experiment part:}
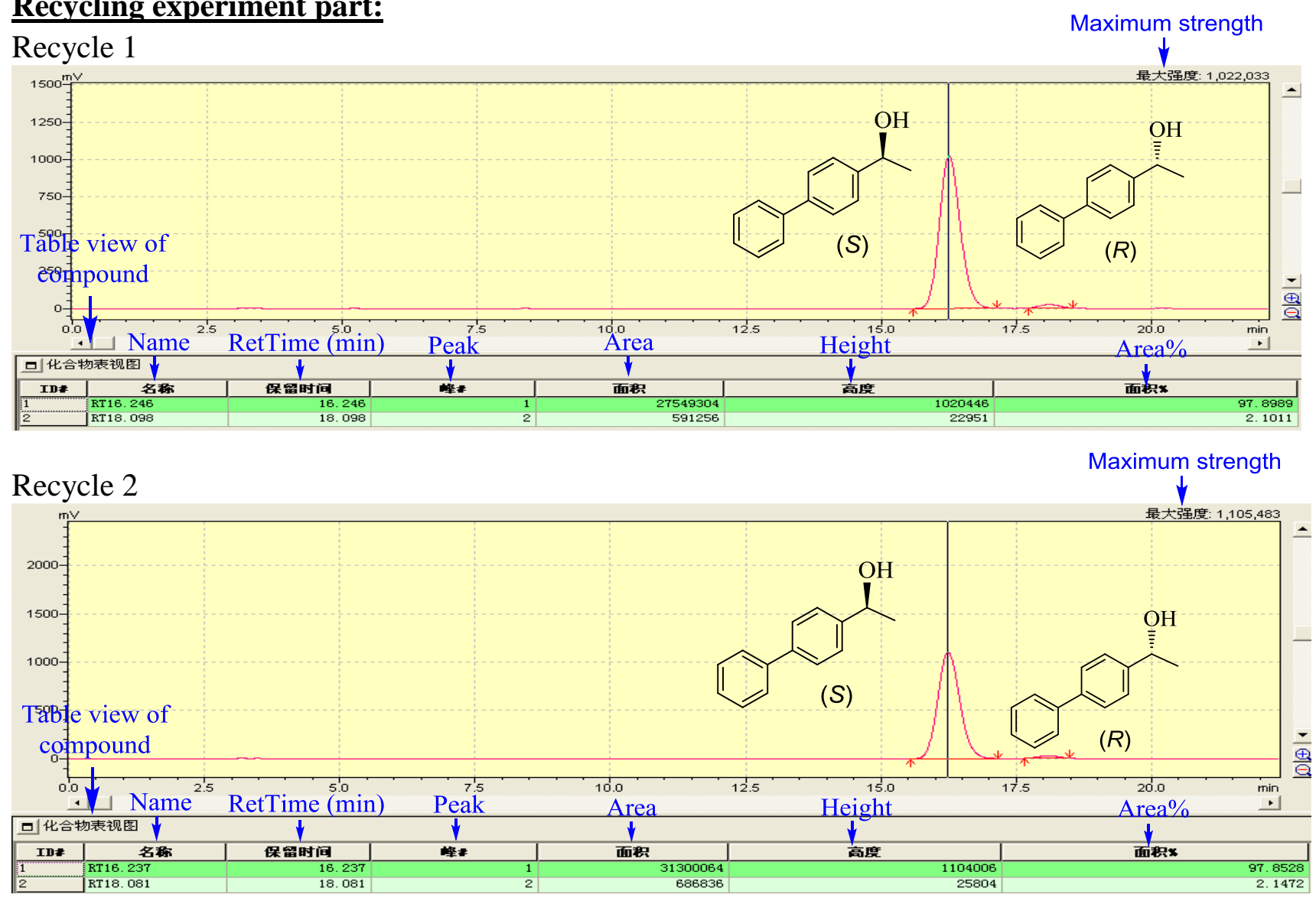

\section{Translation of Chinese into English as follows.}

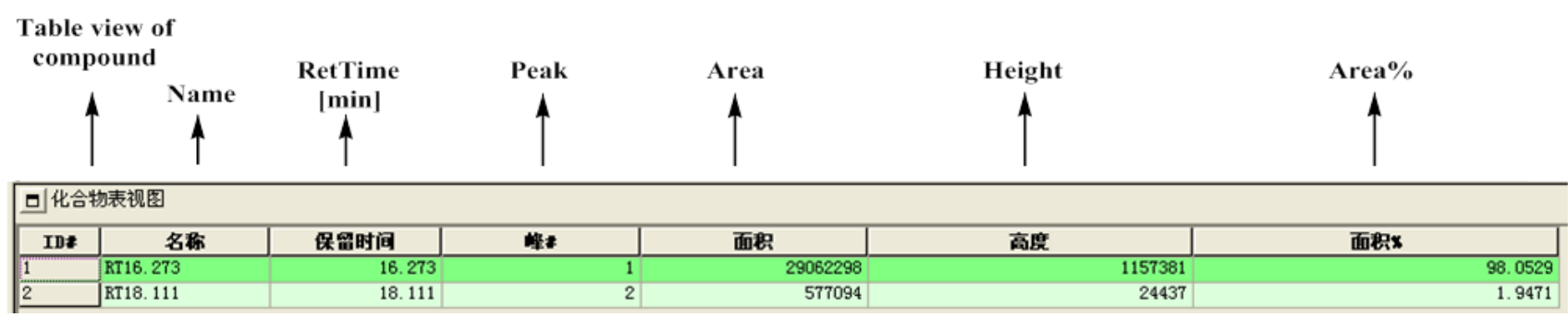


Recycle 3

Maximum strength

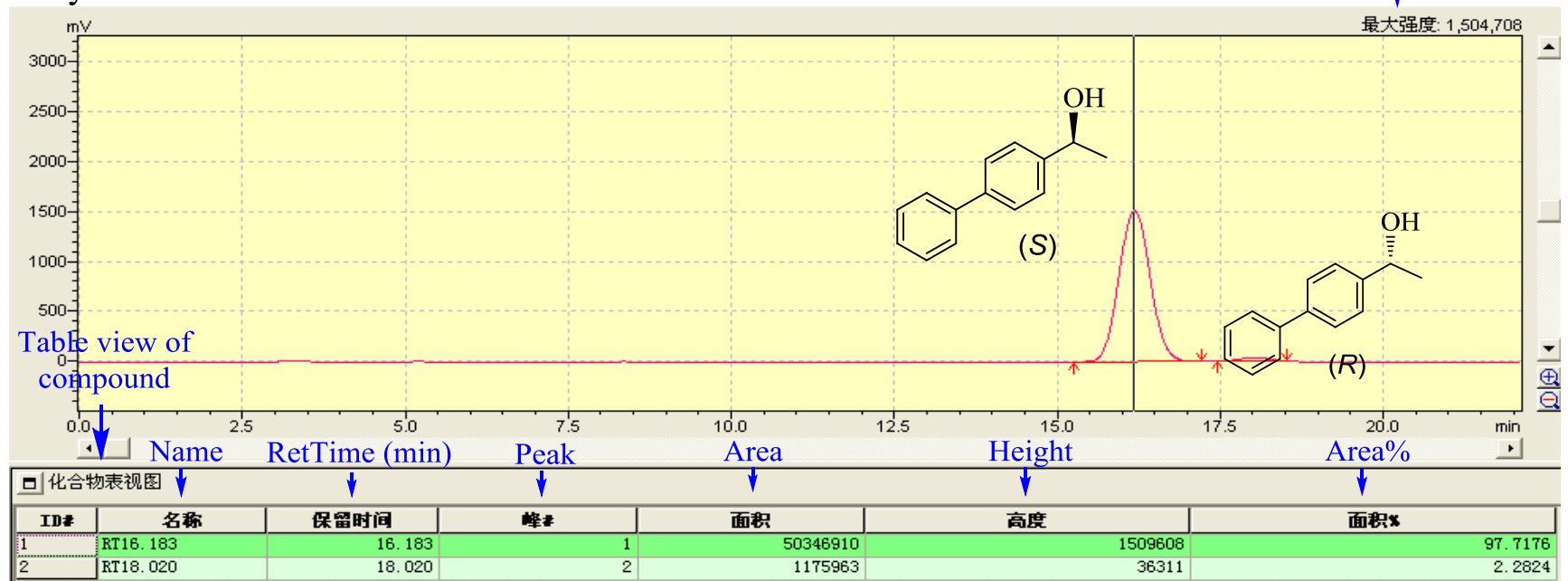

Recycle 4

Maximum strength

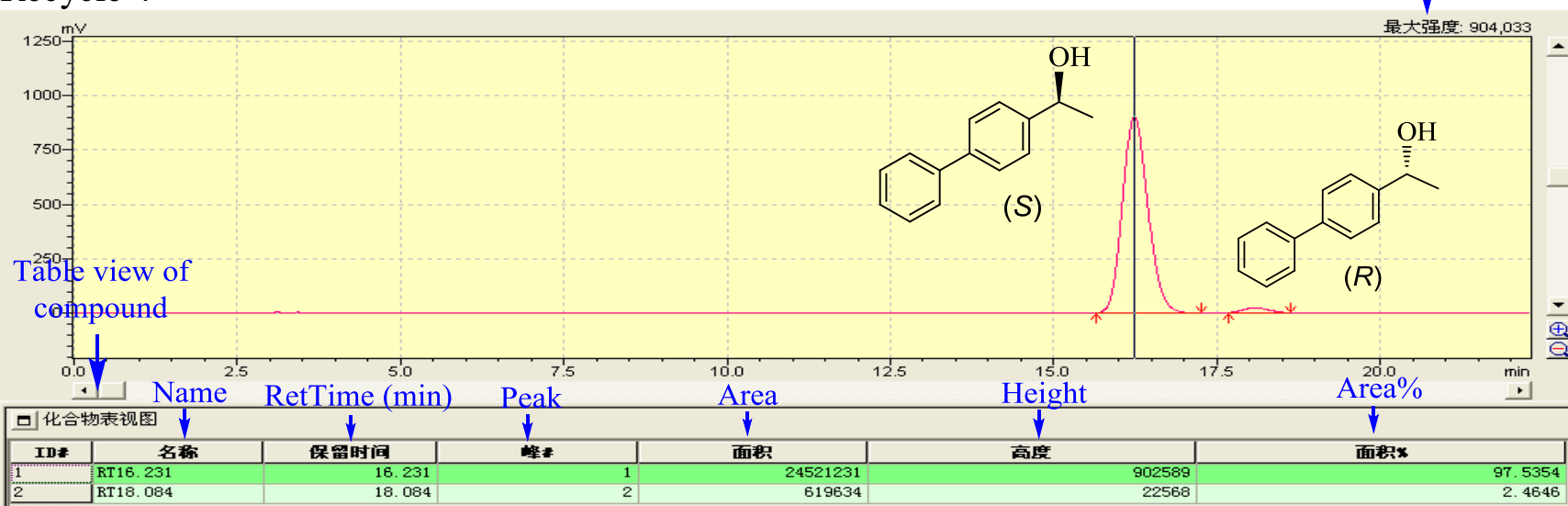

Recycle 5

Maximum strength

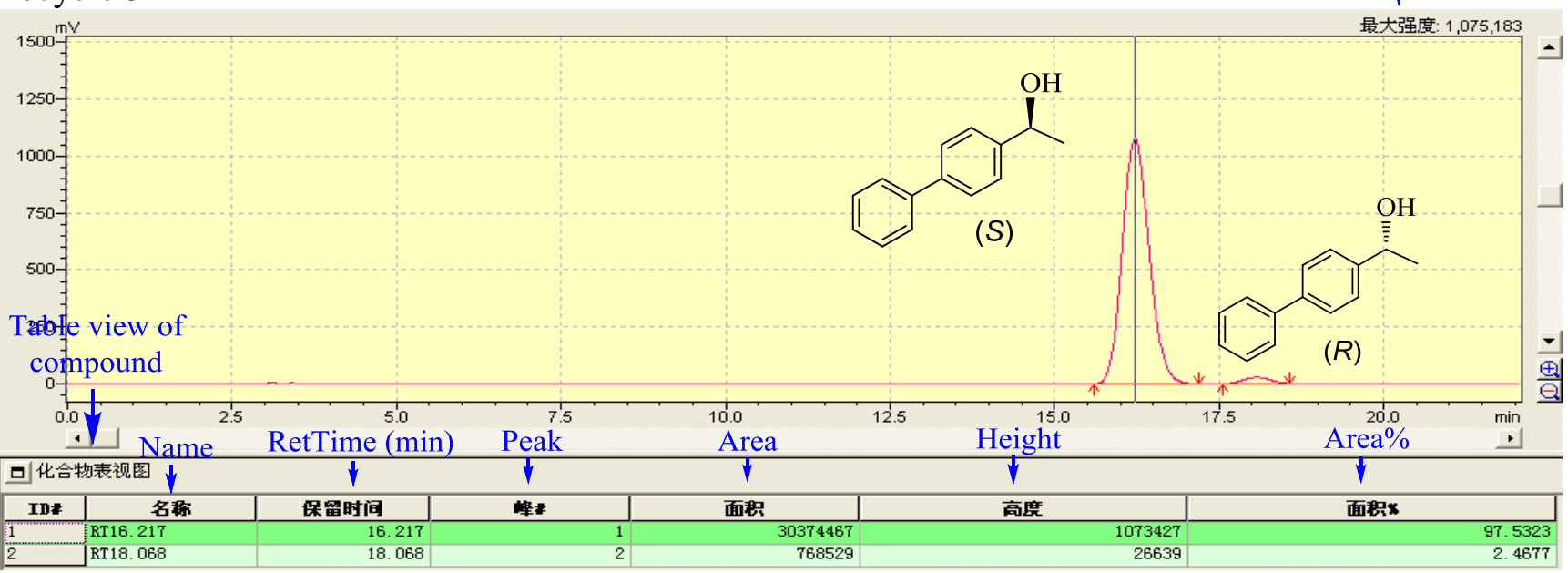

Translation of Chinese into English as follows.

Table view of

compound
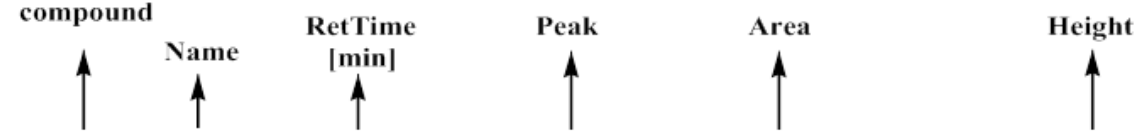

Area \%

\begin{tabular}{|c|c|c|c|c|c|c|}
\hline \multicolumn{7}{|c|}{ 므化合物表视圈 } \\
\hline IDt & 名称 & 保雷时间 & 崔: & 面积 & 高度 & 面积x \\
\hline 1 & RT16.273 & 16.273 & 1 & 29062298 & 1157381 & 98.0529 \\
\hline 2 & RT18.111 & 18.111 & 2 & 577094 & 24437 & 1. 9471 \\
\hline
\end{tabular}


Recycle 6

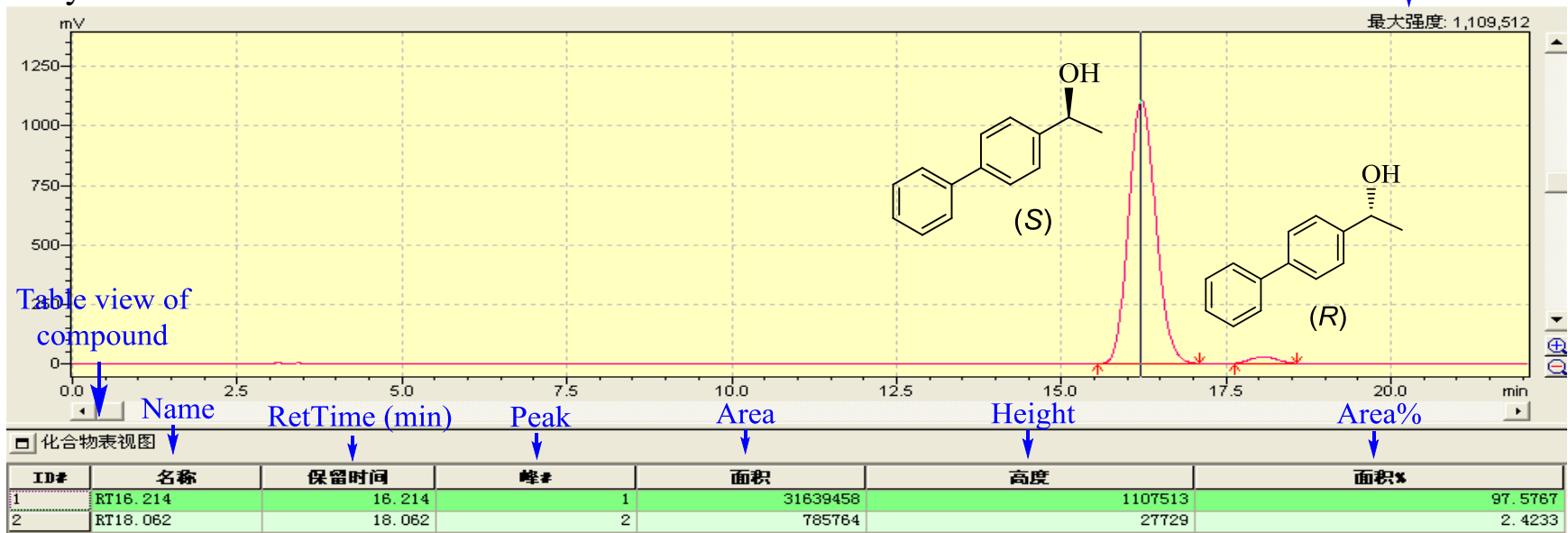

\section{Recycle 7}

Maximum strength

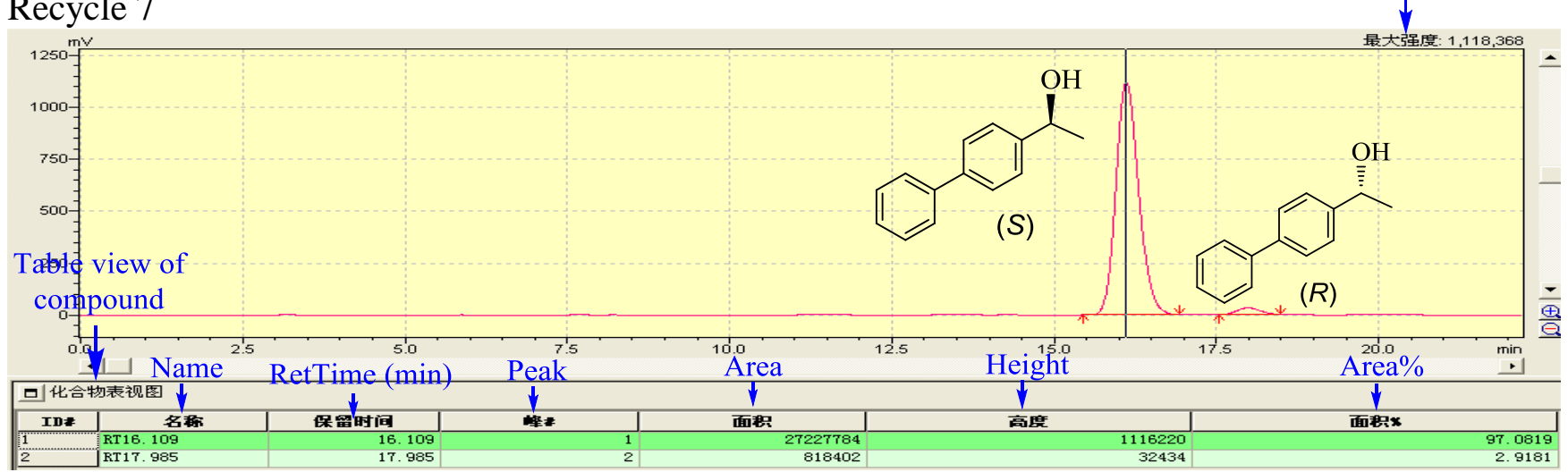

\section{Recycle 8}

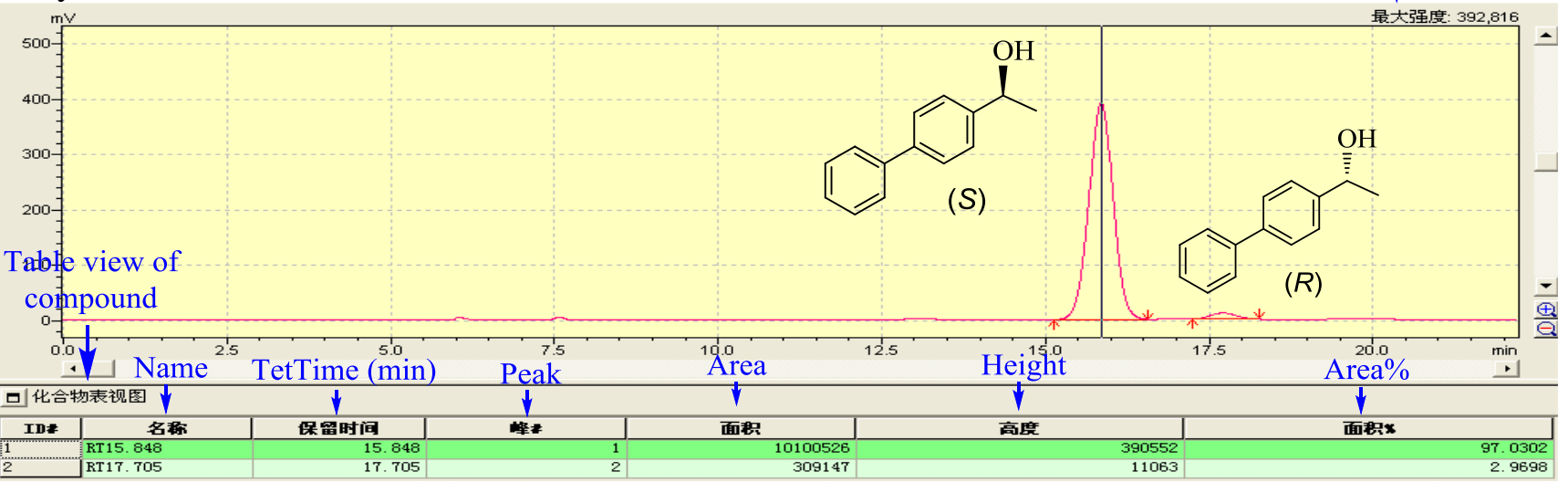

\section{Translation of Chinese into English as follows.}

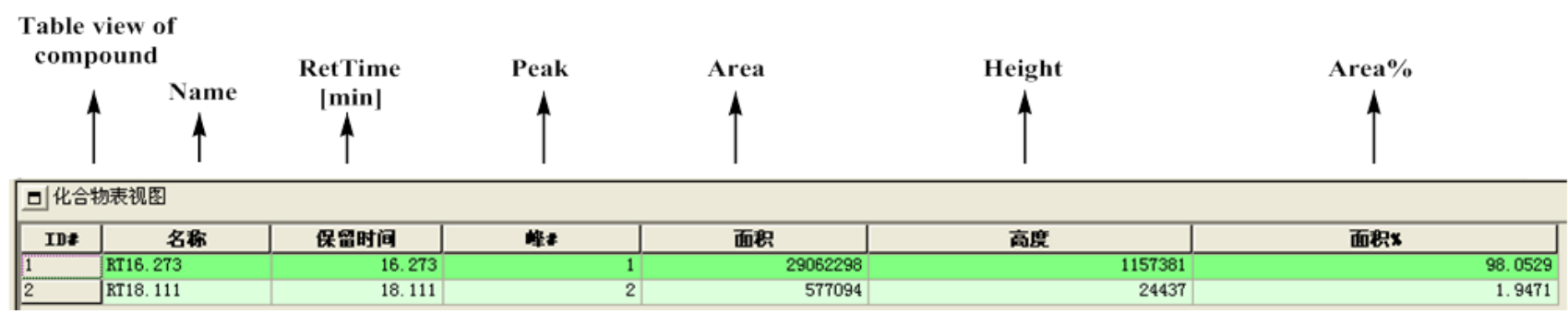

Figure S13. Reusability of catalyst 5 for the Suzuki cross-coupling/ATH of cascade reactions of 4-chloroacetophenone and phenylboronic acid. 
Table S3. Reusability of catalyst 6 for the aza-Michal addition/ATH cascade reaction of 1-phenylprop2-enone and aniline. (Reaction conditions: Catalyst 6 (146.00 mg, $20.0 \mu \mathrm{mol} \mathrm{of} \mathrm{Ru,} \mathrm{based} \mathrm{on} \mathrm{ICP}$ analysis), enones (1.00 mmol), amines $(1.10 \mathrm{mmol}), \mathrm{HCOONa}(10.0 \mathrm{mmol})$, and $20.0 \mathrm{~mL}$ of $\mathrm{H}_{2} \mathrm{O} / \mathrm{PrOH}$ $(\mathrm{v} / \mathrm{v}=1: 1)$ were added sequentially to a $50.0 \mathrm{~mL}$ round-bottom flask. The mixture was then stirred at $15{ }^{\circ} \mathrm{C}$ for the first $2 \mathrm{~h}$ followed by at $35{ }^{\circ} \mathrm{C}$ for $6 \mathrm{~h}$. Yields were determined by ${ }^{1} \mathrm{H}-\mathrm{NMR}$ and $e e$ values were determined chiral HPLC analysis).

\begin{tabular}{lllllllll}
\hline Entry & $\mathbf{1}$ & $\mathbf{2}$ & $\mathbf{3}$ & $\mathbf{4}$ & $\mathbf{5}$ & $\mathbf{6}$ & $\mathbf{7}$ & $\mathbf{8}$ \\
\hline Yield(\%) & 93 & 93 & 91 & 93 & 93 & 91 & 88 & 78 \\
ee $(\%)$ & 94 & 94 & 94 & 94 & 94 & 94 & 94 & 93 \\
\hline
\end{tabular}

\section{$\underline{\text { Recycling experiment part: }}$}

Recycle 1

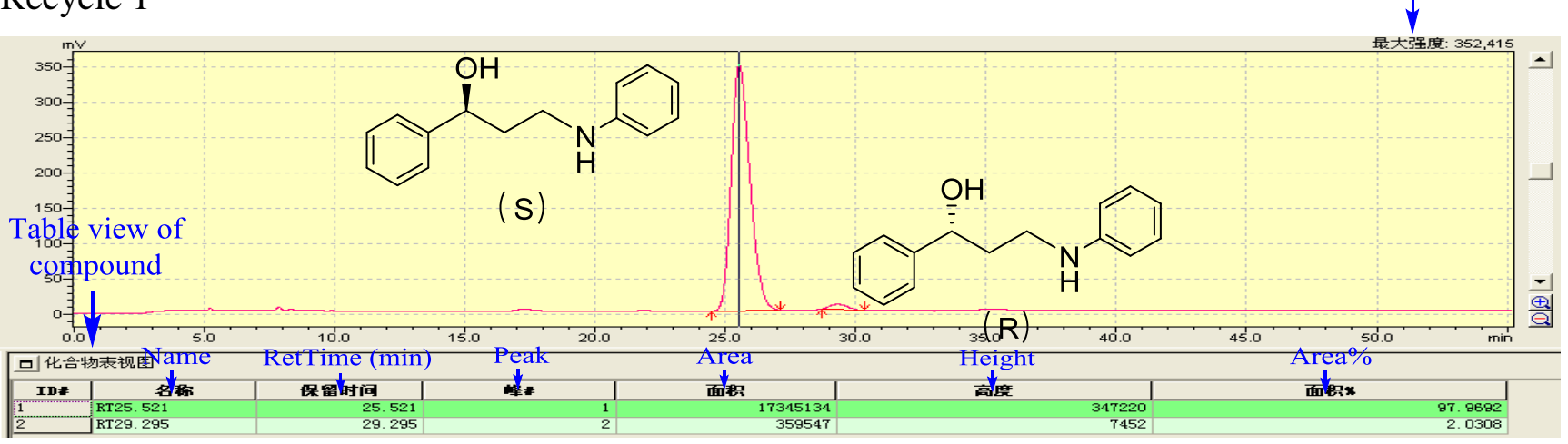

Recycle 2

Maximum strength

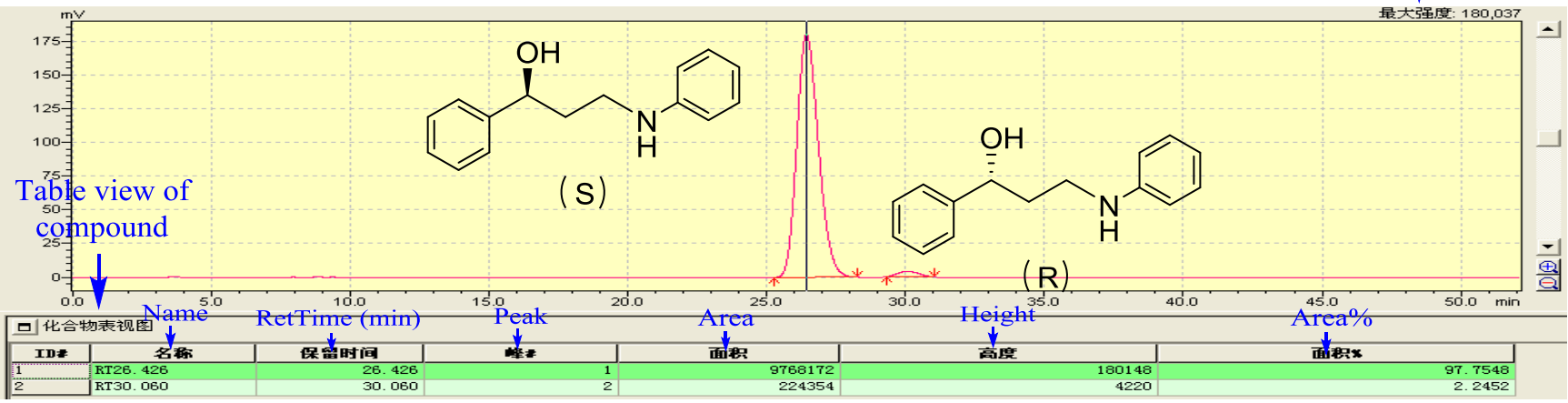

\section{$\underline{\text { Translation of Chinese into English as follows. }}$}

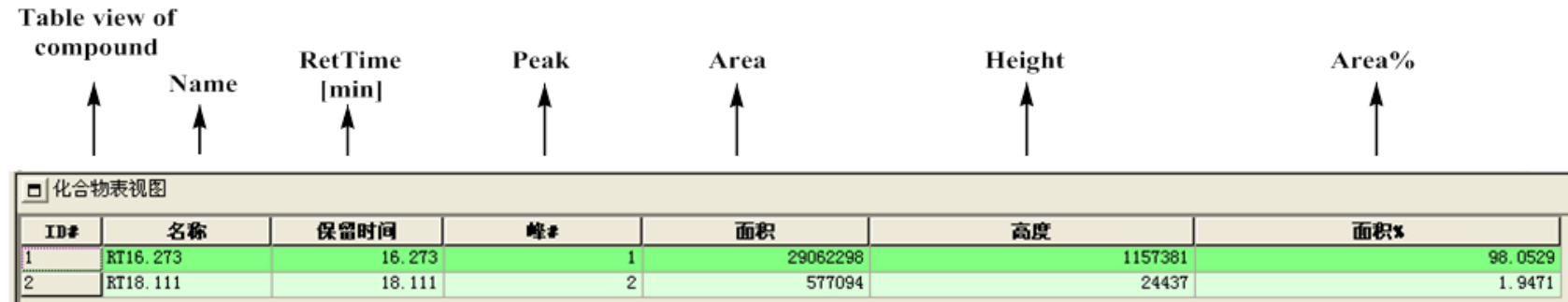


Recycle 3

Maximum strength

$\downarrow$

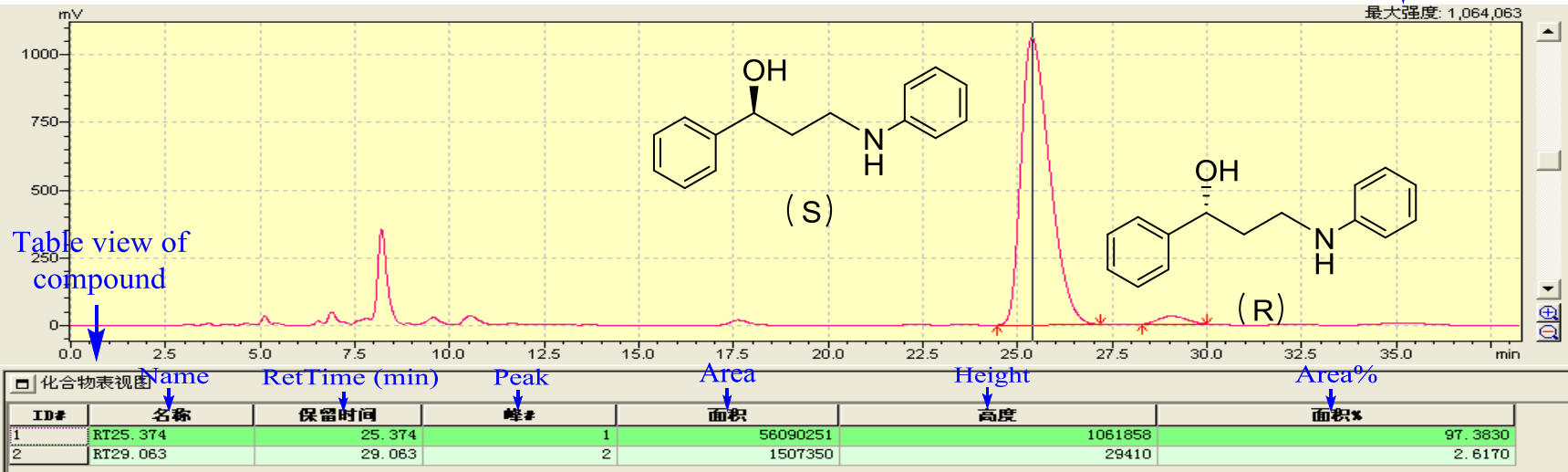

Recycle 4

Maximum strength

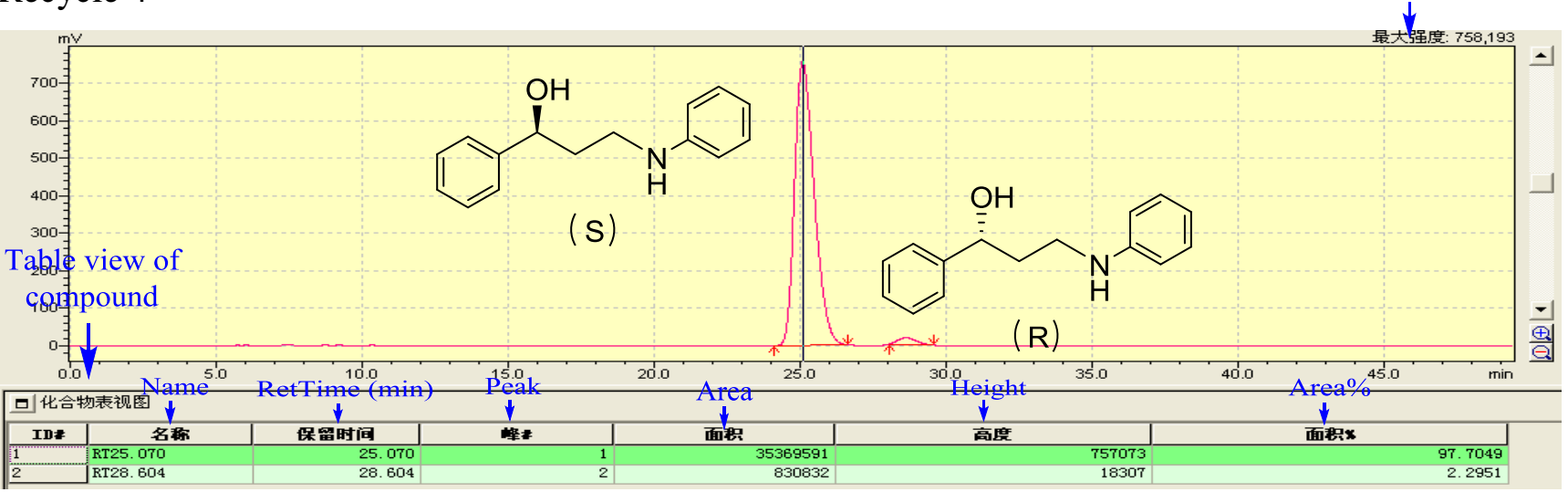

Recycle 5

Maximum strength

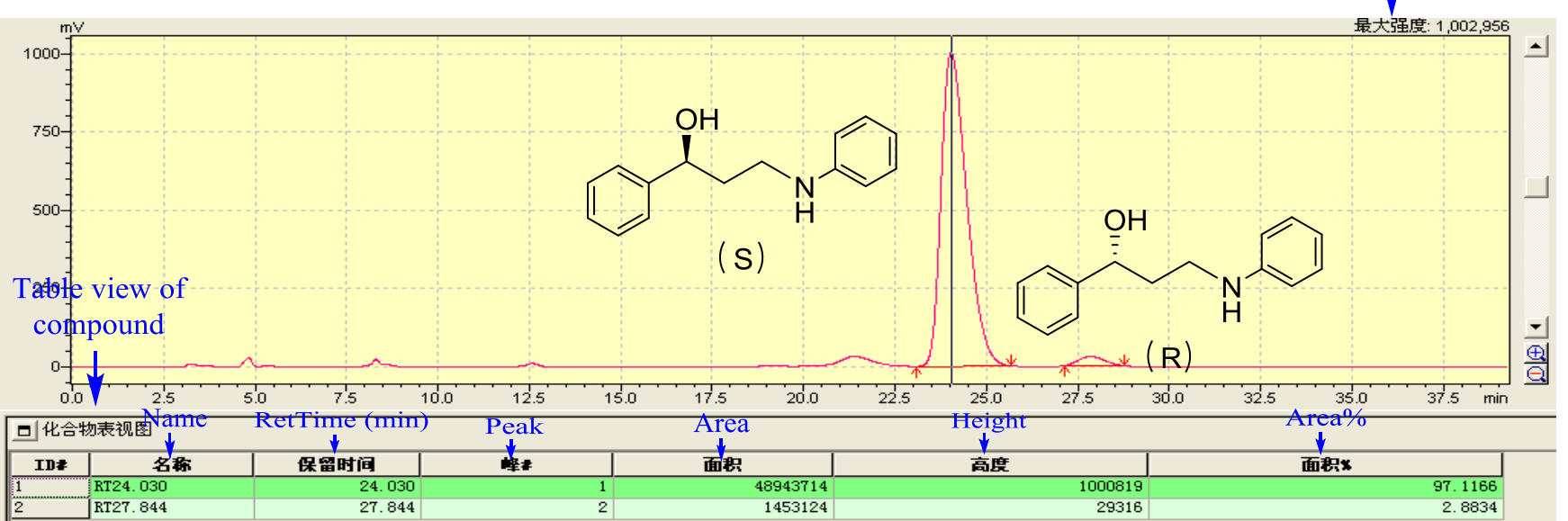

Translation of Chinese into English as follows.

Table view of

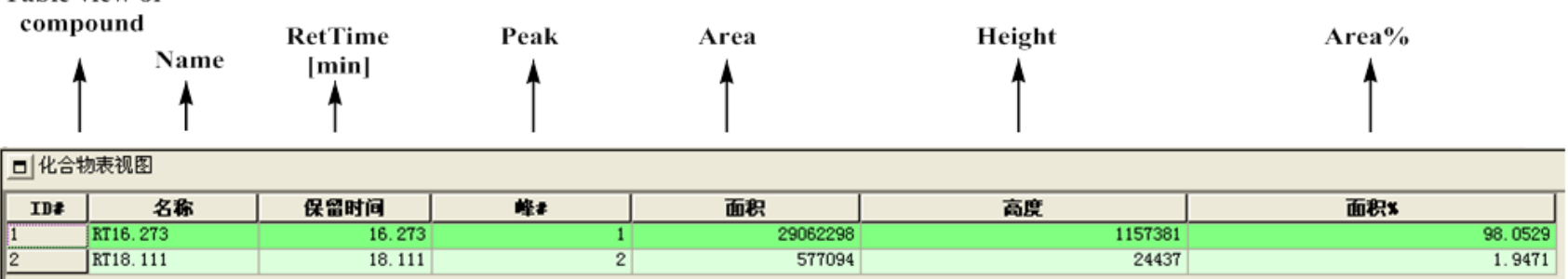


Recycle 6

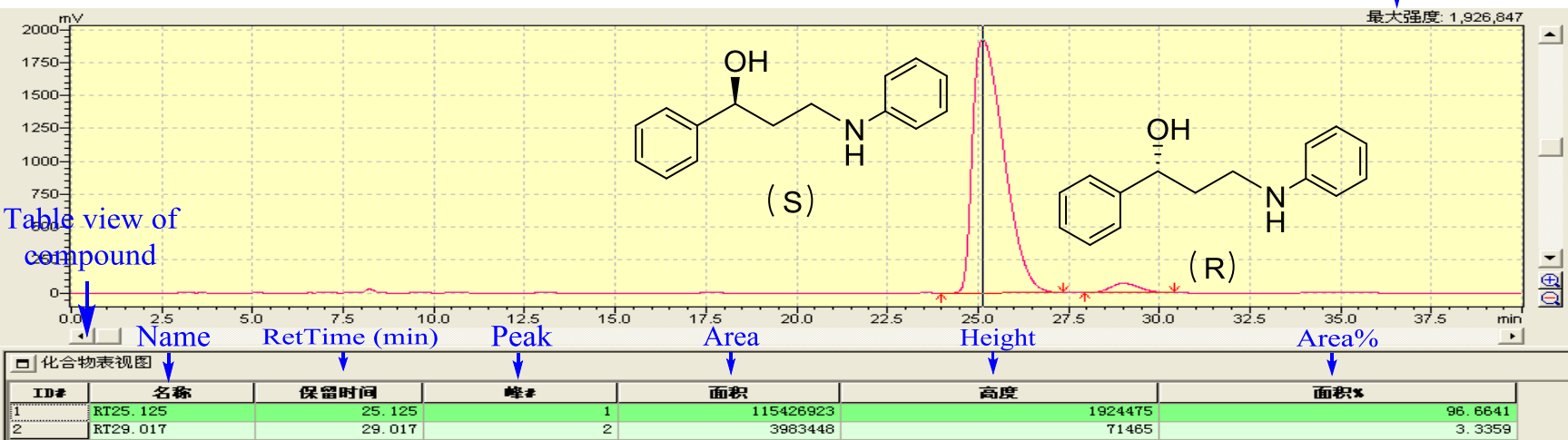

Recycle 7

Maximum strength<smiles>O[C@@H](CCNc1ccccc1)c1ccccc1</smiles>

(s)

Table view of corppound

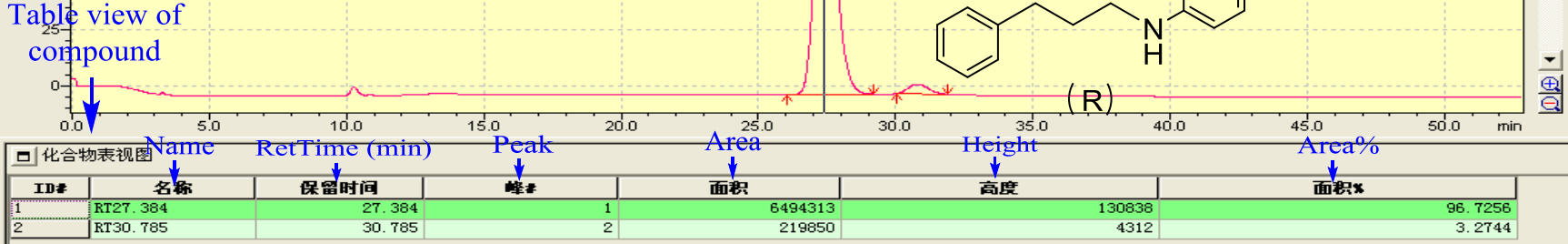

Recycle 8

Maximum strength $\downarrow$

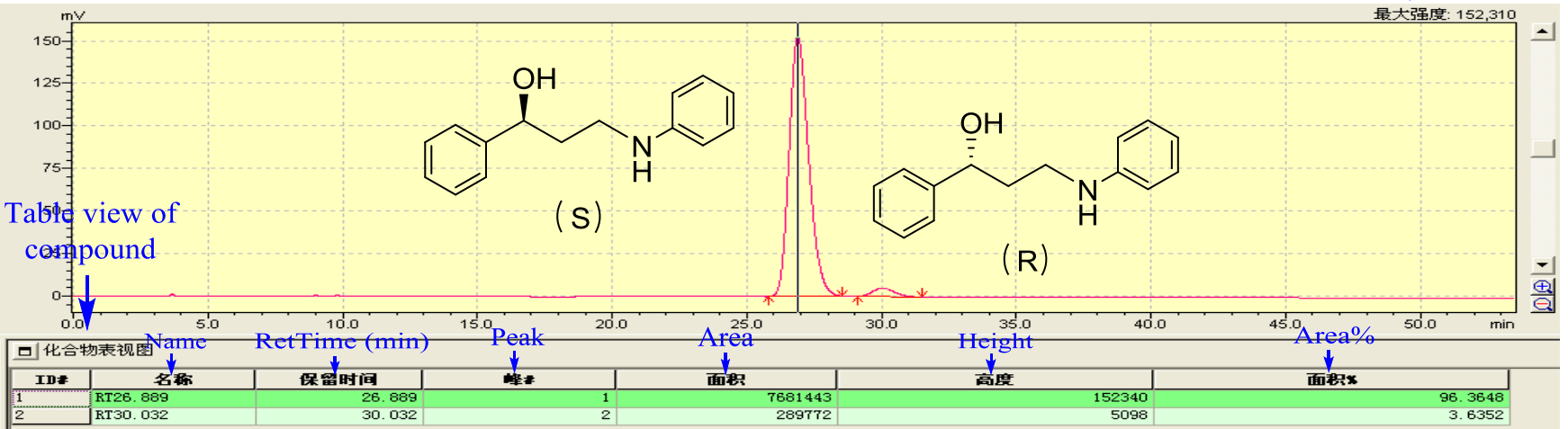

Translation of Chinese into English as follows.

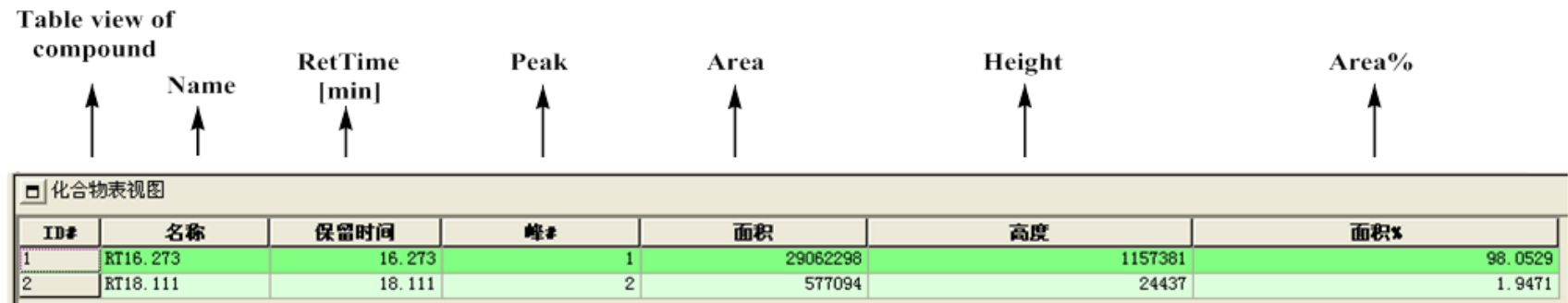

Figure S14. Reusability of catalyst 6 for the aza-Michal addition/ATH cascade reaction of 1phenylprop-2-enone and aniline. 
9a (entry 1 in Table 1): (S)-1-([1,1'-biphenyl]-4-yl)ethan-1-ol $\left({ }^{1} \mathrm{H}\right.$ NMR $\left(400 \mathrm{MHz}, \mathrm{CDCl}_{3}\right) \delta 7.63$ - $7.55(\mathrm{~m}, 3 \mathrm{H}), 7.50-7.40(\mathrm{~m}, 3 \mathrm{H}), 7.40-7.30(\mathrm{~m}, 1 \mathrm{H}), 7.26(\mathrm{~s}, 2 \mathrm{H}), 4.97$ (q, J = 6.5 Hz, 1H), 1.55 (d, $\mathrm{J}=6.5 \mathrm{~Hz}, 3 \mathrm{H}) . \mathrm{GC} / \mathrm{MS}(\mathrm{m} / \mathrm{z}): 198)$.
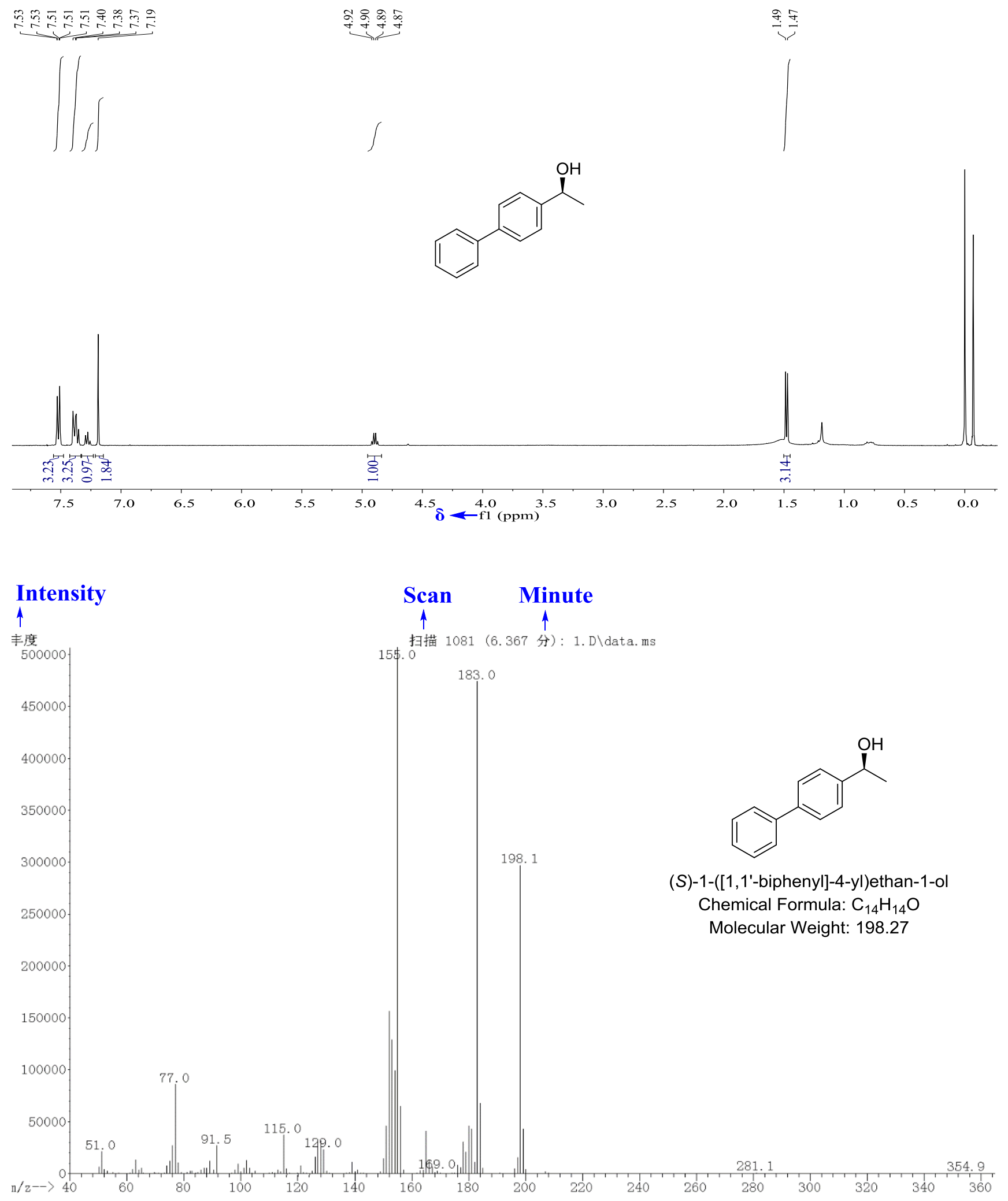
$9 b$ (entry 2 in Table 1): (S)-1-(4'-fluoro-[1,1'-biphenyl]-4-yl)ethan-1-ol ( ${ }^{1} \mathrm{H}$ NMR (400 MHz, Chloroform-d) $\delta 7.47(\mathrm{dd}, \mathrm{J}=8.5,5.0 \mathrm{~Hz}, 4 \mathrm{H}), 7.38(\mathrm{~d}, \mathrm{~J}=8.2 \mathrm{~Hz}, 2 \mathrm{H}), 7.05$ (t, J = 8.7 Hz, 2H), 4.89 (q, $\mathrm{J}=6.5 \mathrm{~Hz}, 1 \mathrm{H}), 1.47(\mathrm{~d}, \mathrm{~J}=6.5 \mathrm{~Hz}, 3 \mathrm{H}) . \mathrm{GC} / \mathrm{MS}(\mathrm{m} / \mathrm{z}): 216)$.
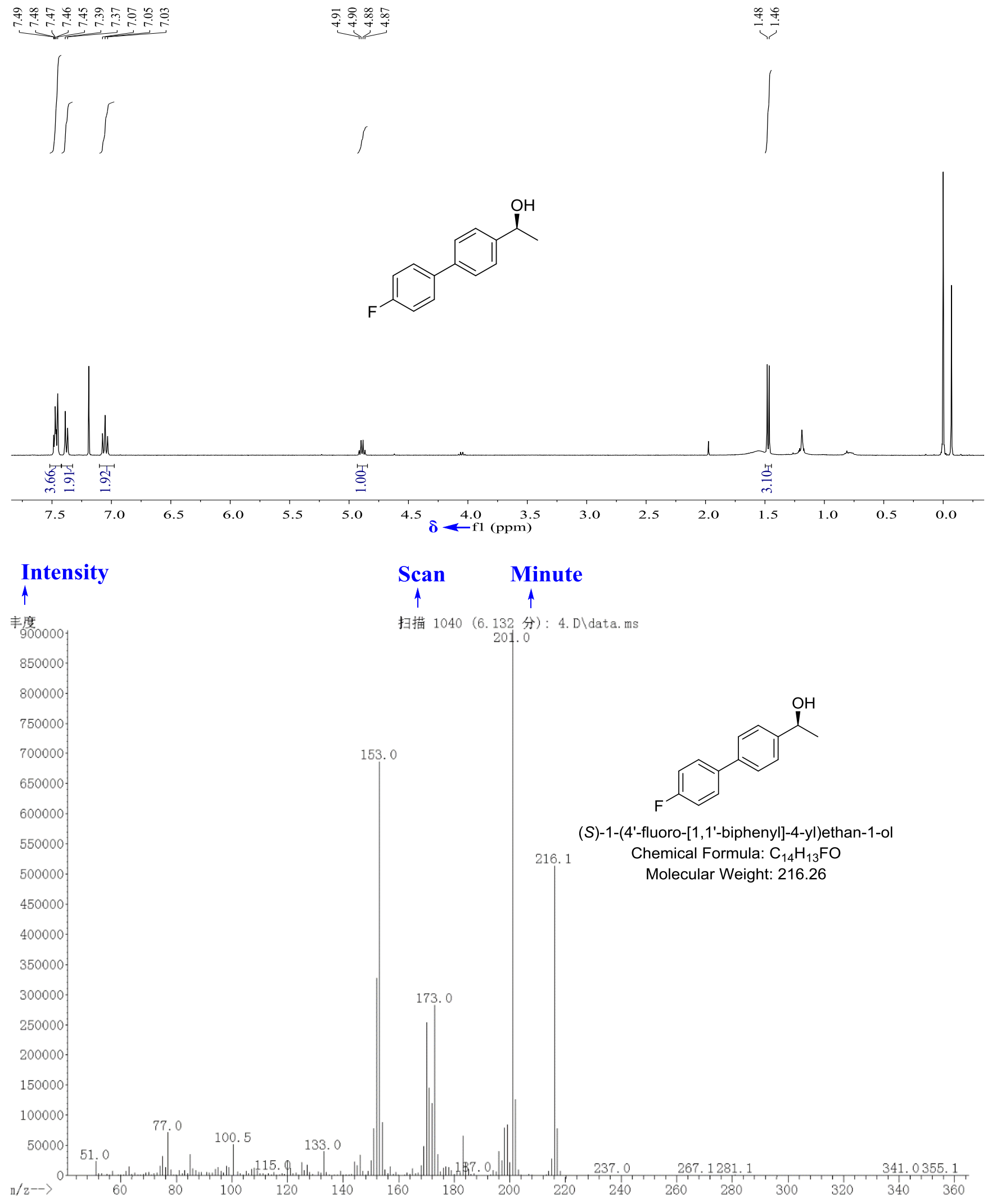
9 c (entry 3 in Table 1): (S)-1-(4'-chloro-[1,1'-biphenyl]-4-yl)ethan-1-ol $\left({ }^{1} \mathrm{H}\right.$ NMR (400 MHz, Chloroform-d) $\delta 7.46(\mathrm{dd}, \mathrm{J}=13.1,8.5 \mathrm{~Hz}, 4 \mathrm{H}), 7.39$ (d, J = 8.2 Hz, 2H), 7.33 (d, J = 8.5 Hz, 2H), 4.89 $(\mathrm{q}, \mathrm{J}=6.5 \mathrm{~Hz}, 1 \mathrm{H}), 1.47(\mathrm{~d}, \mathrm{~J}=6.5 \mathrm{~Hz}, 3 \mathrm{H}) . \mathrm{GC} / \mathrm{MS}(\mathrm{m} / \mathrm{z}): 232)$.
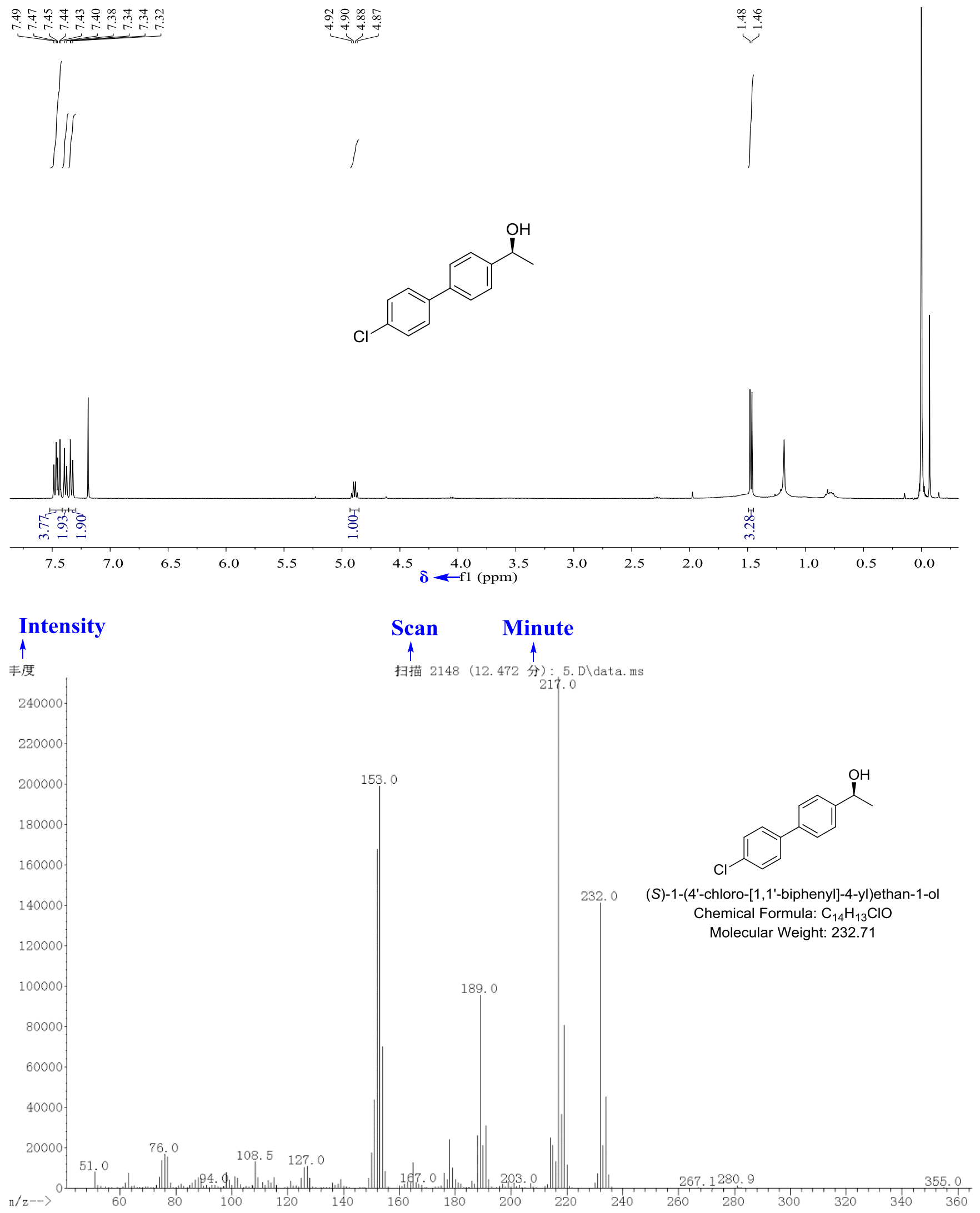
9d (entry 4 in Table 1): (S)-1-(3'-chloro-[1,1'-biphenyl]-4-yl)ethan-1-ol ( ${ }^{1} \mathrm{H}$ NMR (400 MHz, Chloroform-d) $\delta 7.58(\mathrm{dd}, \mathrm{J}=8.9,2.3 \mathrm{~Hz}, 3 \mathrm{H}), 7.52-7.45(\mathrm{~m}, 3 \mathrm{H}), 7.43-7.31(\mathrm{~m}, 2 \mathrm{H}), 4.99(\mathrm{q}, \mathrm{J}=$ $6.5 \mathrm{~Hz}, 1 \mathrm{H}), 1.57$ (d, J = 6.5 Hz, 3H).GC/MS (m/z): 232).
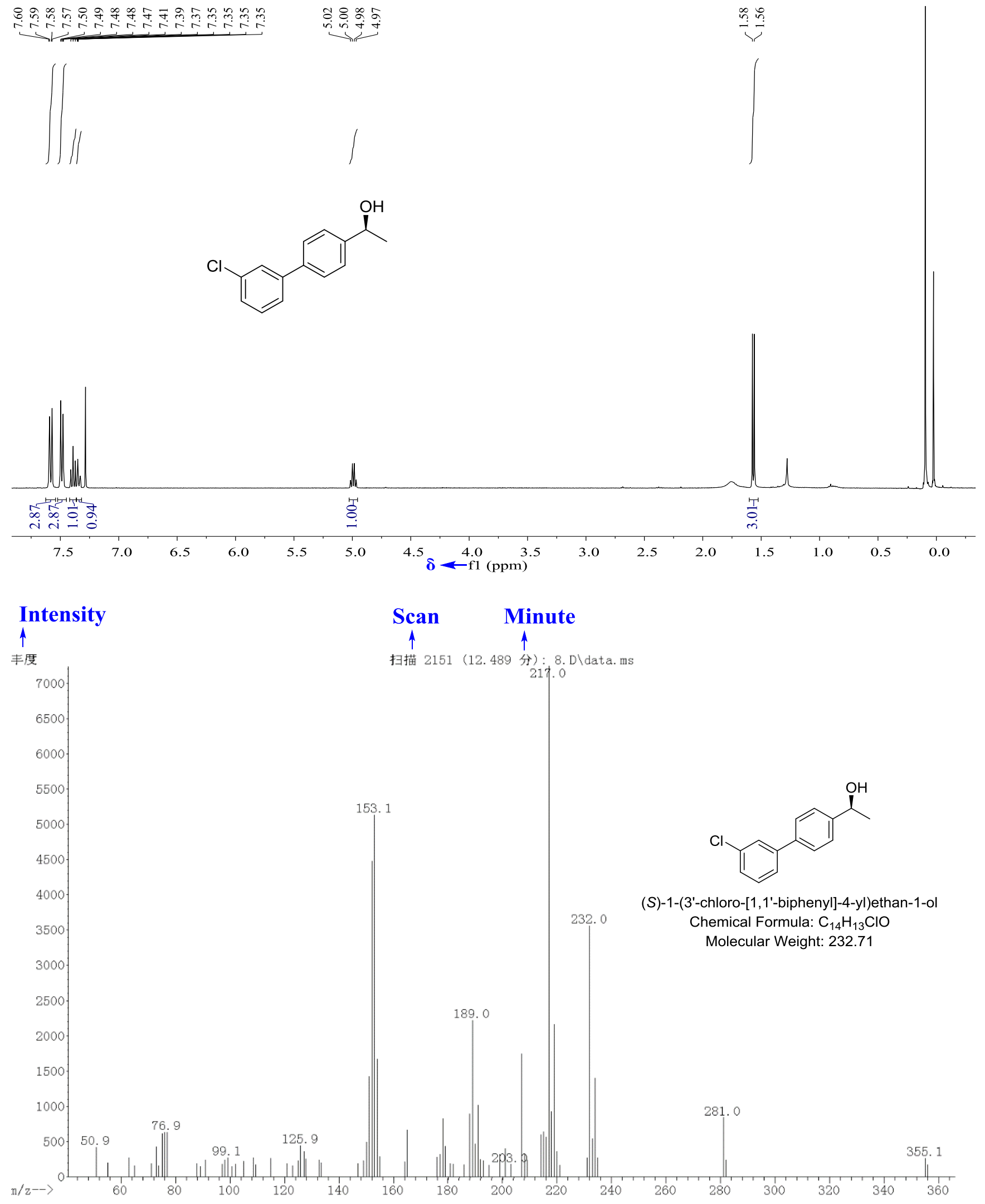
9e (entry 5 in Table 1): (S)-1-(4'-(trifluoromethyl)-[1,1'-biphenyl]-4-yl)ethan-1-ol ( ${ }^{1} \mathrm{H}$ NMR (400 $\mathrm{MHz}$, Chloroform-d) $\delta 7.62(\mathrm{~d}, \mathrm{~J}=0.9 \mathrm{~Hz}, 4 \mathrm{H}), 7.56-7.49(\mathrm{~m}, 2 \mathrm{H}), 7.42(\mathrm{~d}, \mathrm{~J}=8.2 \mathrm{~Hz}, 2 \mathrm{H}), 4.91(\mathrm{q}, \mathrm{J}$ $=6.4 \mathrm{~Hz}, 1 \mathrm{H}), 1.48(\mathrm{~d}, \mathrm{~J}=6.5 \mathrm{~Hz}, 3 \mathrm{H}) \cdot \mathrm{GC} / \mathrm{MS}(\mathrm{m} / \mathrm{z}): 266)$.
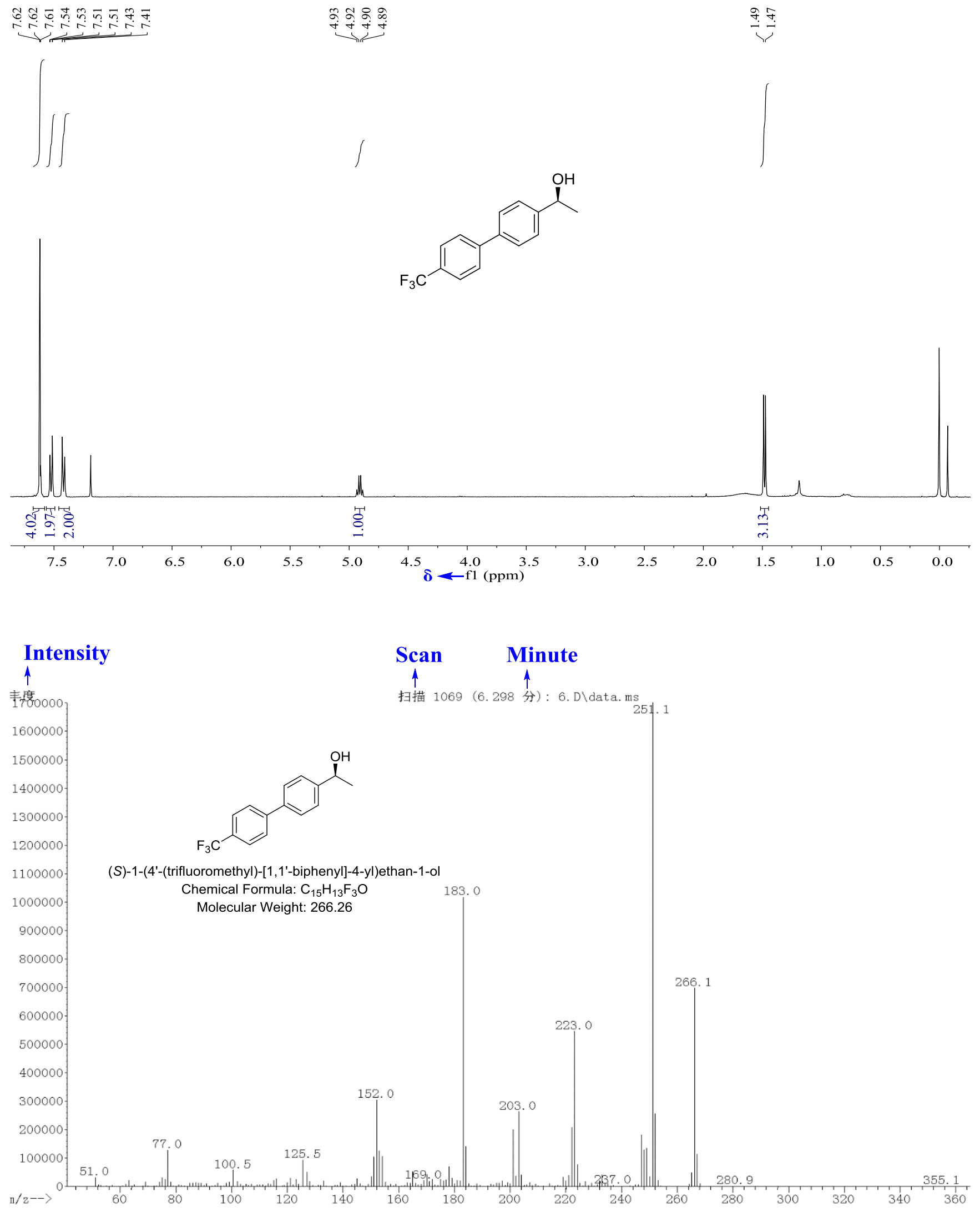
9f(entry 6 in Table 1): (S)-1-(3'-(trifluoromethyl)-[1,1'-biphenyl]-4-yl)ethan-1-ol ( ${ }^{1} \mathrm{H}$ NMR (400 MHz, Chloroform-d) $\delta 7.76$ (s, 1H), 7.69 (d, J = 7.5 Hz, 1H), $7.57-7.45$ (m, 4H), 7.42 (d, J = 8.2 Hz, 2H), $4.91(\mathrm{q}, \mathrm{J}=6.5 \mathrm{~Hz}, 1 \mathrm{H}), 1.48(\mathrm{~d}, \mathrm{~J}=6.5 \mathrm{~Hz}, 3 \mathrm{H}) . \mathrm{GC} / \mathrm{MS}(\mathrm{m} / \mathrm{z}): 266)$.
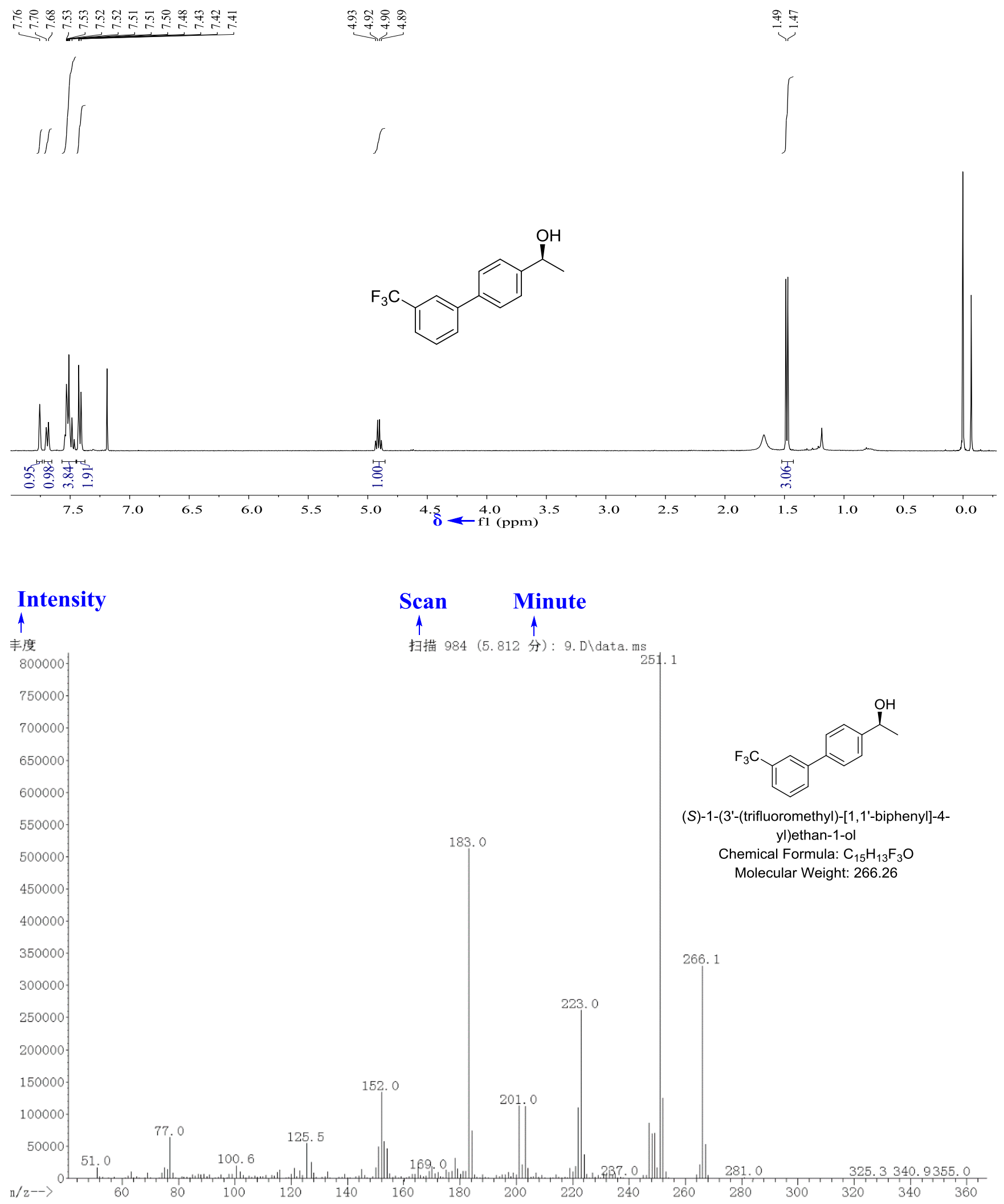
$9 g$ (entry 7 in Table 1): (S)-1-(4'-methyl-[1,1'-biphenyl]-4-yl)ethan-1-ol ( ${ }^{1} \mathrm{H}$ NMR (400 MHz, Chloroform-d) $\delta$ 7.54-7.47 (m, 2H), $7.45-7.40(\mathrm{~m}, 2 \mathrm{H}), 7.39-7.34(\mathrm{~m}, 2 \mathrm{H}), 7.18(\mathrm{~d}, \mathrm{~J}=8.1 \mathrm{~Hz}, 2 \mathrm{H})$, $4.88(\mathrm{q}, \mathrm{J}=6.5 \mathrm{~Hz}, 1 \mathrm{H}), 2.33(\mathrm{~s}, 3 \mathrm{H}), 1.47$ (d, J = 6.5 Hz, 3H). GC/MS (m/z): 212).
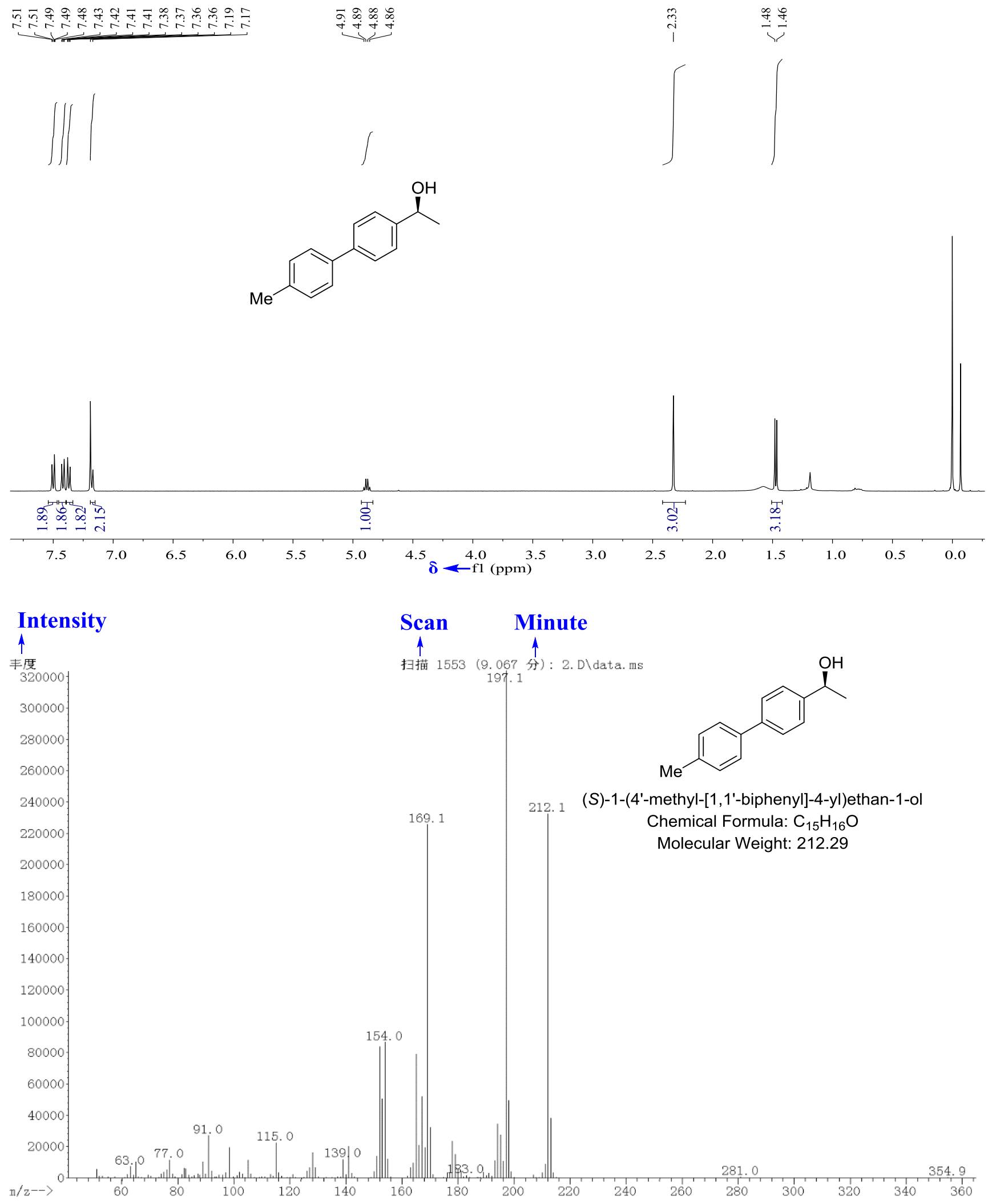
9h (entry 8 in Table 1): (S)-1-(3'-methyl-[1,1'-biphenyl]-4-yl)ethan-1-ol $\left({ }^{1} \mathrm{H}\right.$ NMR (400 MHz,

Chloroform-d) $\delta 7.51$ (d, J = 8.1 Hz, 2H), 7.37 (d, J = 8.2 Hz, 2H), $7.35-7.29$ (m, 2H), 7.26 (t, J = 7.5 $\mathrm{Hz}, 1 \mathrm{H}), 7.10(\mathrm{~d}, \mathrm{~J}=7.3 \mathrm{~Hz}, 1 \mathrm{H}), 4.89$ (q, J = $6.5 \mathrm{~Hz}, 1 \mathrm{H}), 2.35$ (s, 3H), 1.48 (d, J = $6.4 \mathrm{~Hz}, 3 \mathrm{H})$. GC/MS (m/z): 212).
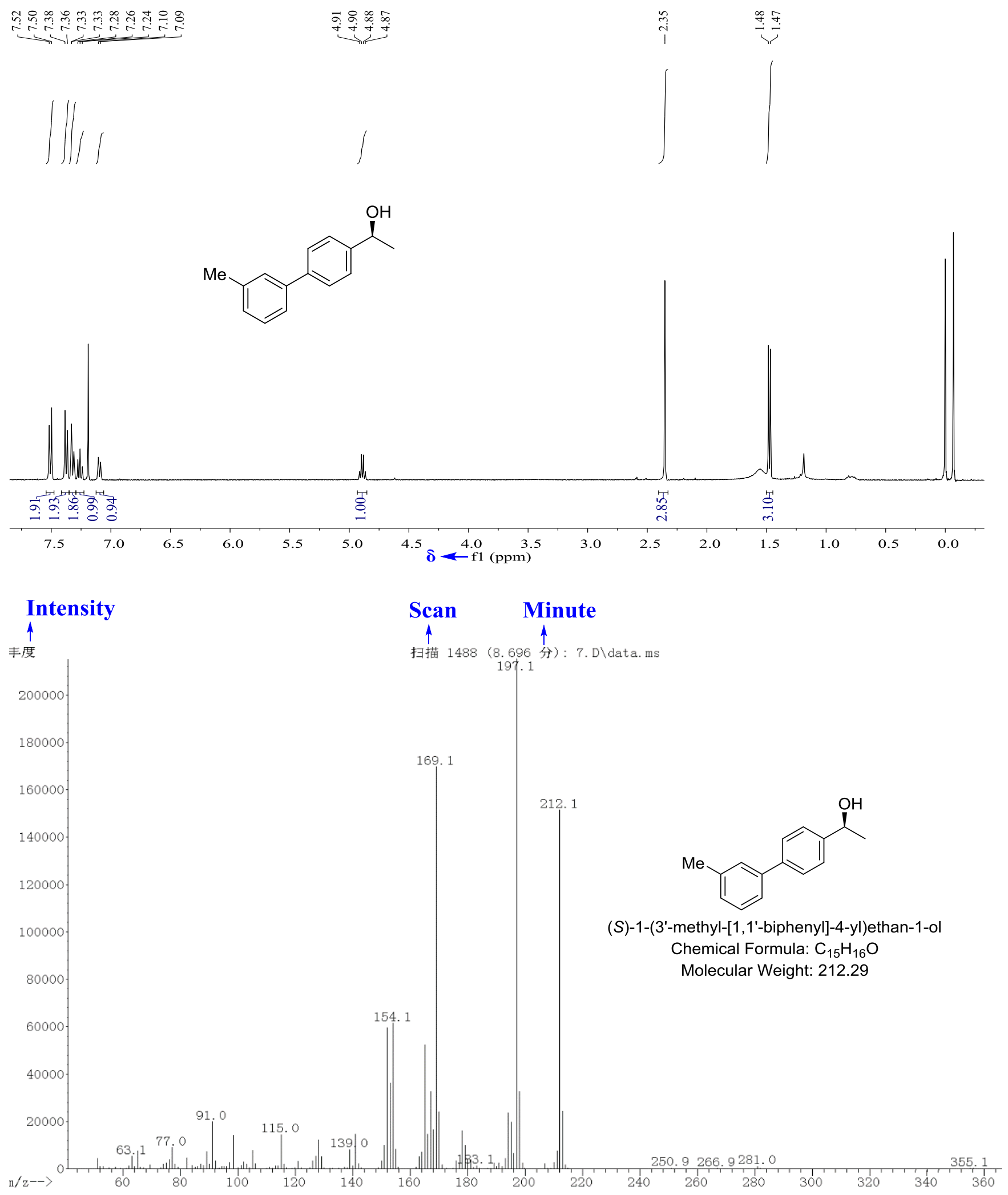
9i (entry 9 in Table 1): (S)-1-(4'-methoxy-[1,1'-biphenyl]-4-yl)ethan-1-ol $\left({ }^{1} \mathrm{H}\right.$ NMR (400 MHz, Chloroform-d) $\delta 7.50-7.43(\mathrm{~m}, 4 \mathrm{H}), 7.36(\mathrm{~d}, \mathrm{~J}=8.3 \mathrm{~Hz}, 2 \mathrm{H}), 6.91(\mathrm{~d}, \mathrm{~J}=8.5 \mathrm{~Hz}, 2 \mathrm{H}), 4.88(\mathrm{q}, \mathrm{J}=6.4$ $\mathrm{Hz}, 1 \mathrm{H}), 3.78$ (s, 3H), 1.47 (d, J = 6.4 Hz, 3H). GC/MS (m/z): 228).
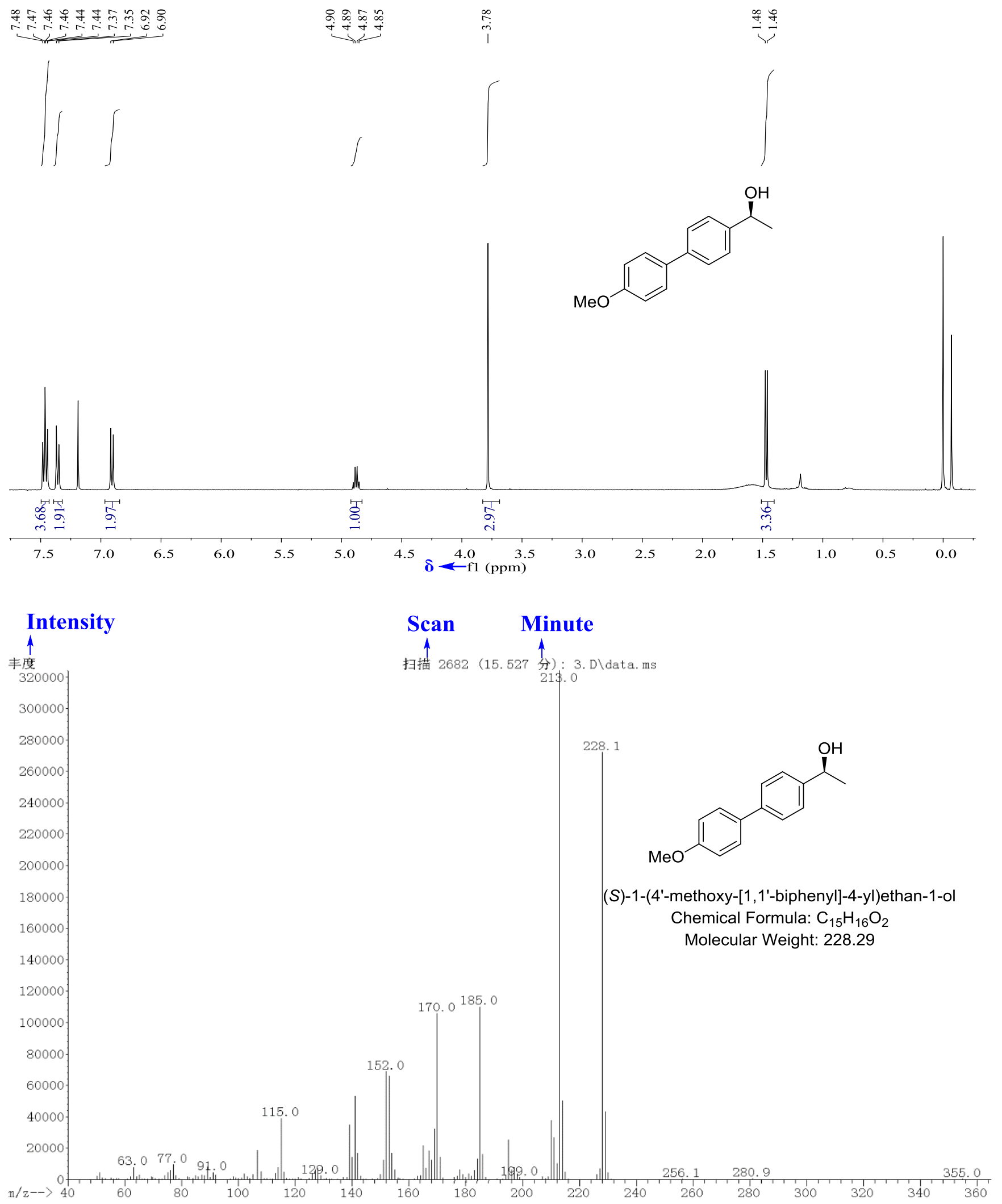
9j (entry 10 in Table 1): (S)-1-(4-(thiophen-3-yl)phenyl)ethanol ( ${ }^{1} \mathrm{H}$ NMR (400 MHz, Chloroformd) $\delta 7.52(\mathrm{~d}, \mathrm{~J}=8.2 \mathrm{~Hz}, 2 \mathrm{H}), 7.40-7.30(\mathrm{~m}, 5 \mathrm{H}), 4.87(\mathrm{q}, \mathrm{J}=6.4 \mathrm{~Hz}, 1 \mathrm{H}), 1.46(\mathrm{~d}, \mathrm{~J}=6.5 \mathrm{~Hz}, 3 \mathrm{H})$. GC/MS (m/z): 204).
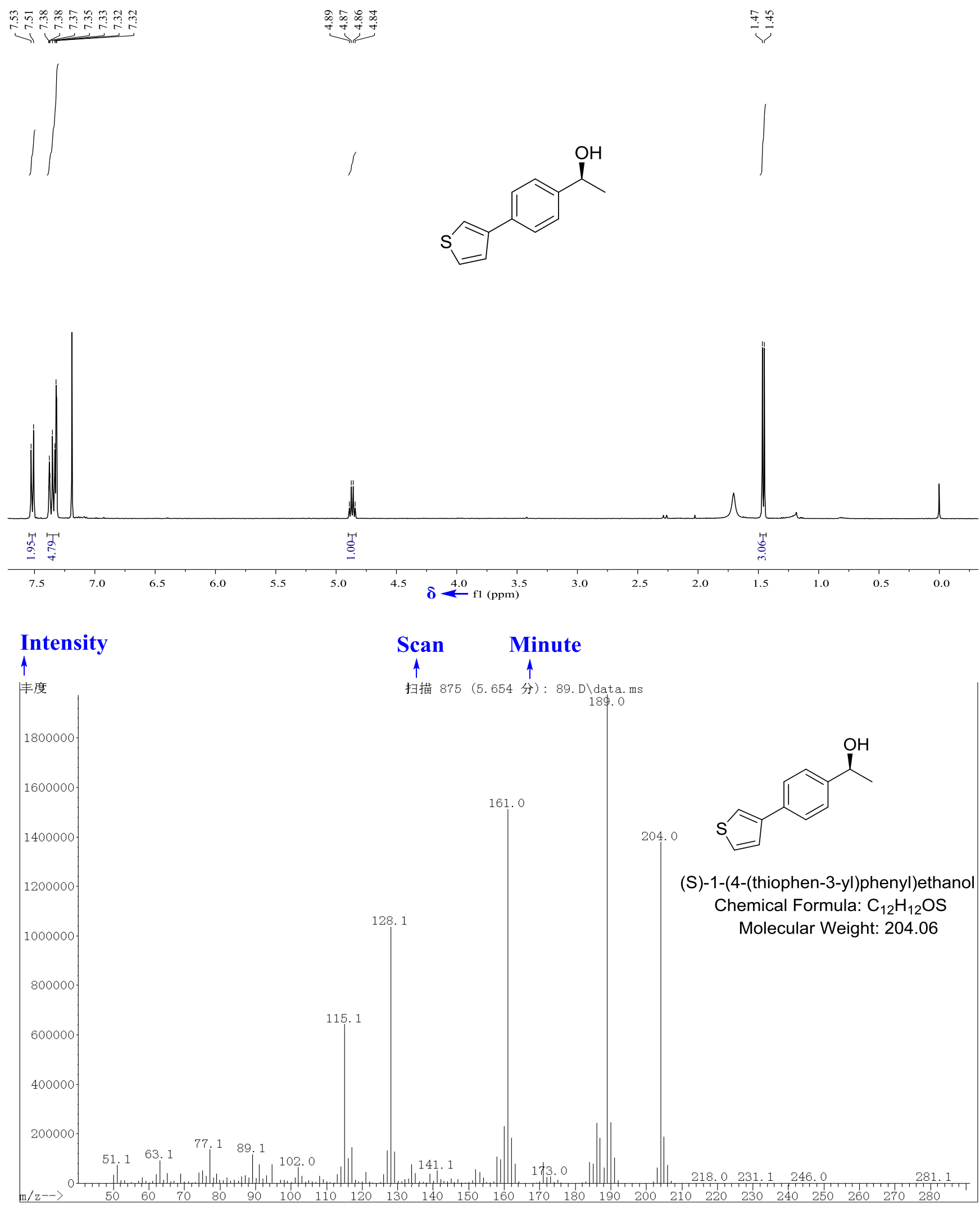
9k (entry 11 in Table 1): (S)-1-(4-(pyridin-4-yl)phenyl)ethanol ( ${ }^{1} \mathrm{H}$ NMR (400 MHz, Chloroform-d) $\delta 8.59(\mathrm{~d}, \mathrm{~J}=5.9 \mathrm{~Hz}, 2 \mathrm{H}), 7.62-7.51(\mathrm{~m}, 4 \mathrm{H}), 7.46(\mathrm{~d}, \mathrm{~J}=8.2 \mathrm{~Hz}, 2 \mathrm{H}), 4.92(\mathrm{q}, \mathrm{J}=6.5 \mathrm{~Hz}, 1 \mathrm{H}), 1.48$ $(\mathrm{d}, \mathrm{J}=6.5 \mathrm{~Hz}, 3 \mathrm{H}) . \mathrm{GC} / \mathrm{MS}(\mathrm{m} / \mathrm{z}): 199)$.
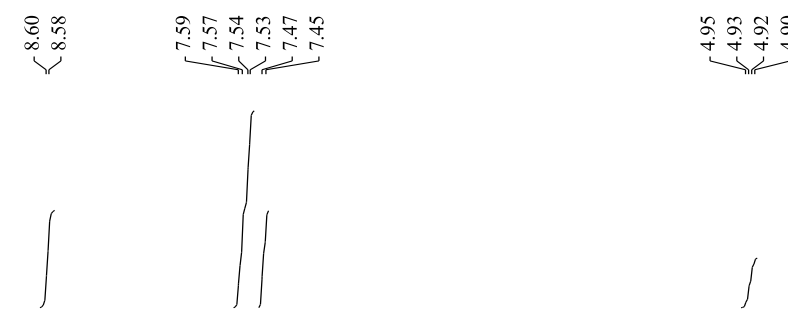<smiles>CC(O)c1ccc(-c2ccncc2)cc1</smiles>
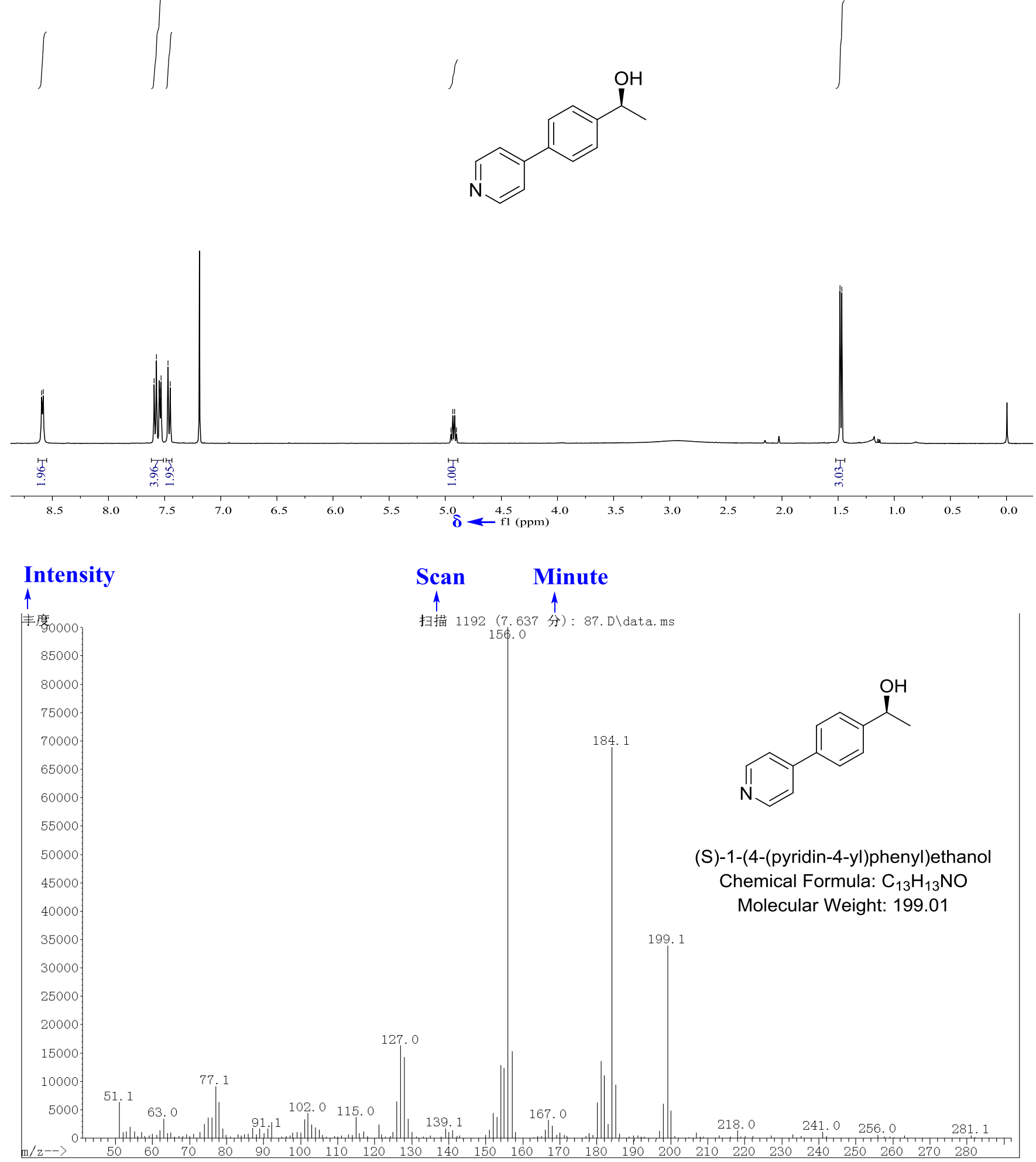
$9 l$ (entry 12 in Table 1): (S)-1-([1,1'-biphenyl]-3-yl)ethan-1-ol ( ${ }^{1} \mathrm{H}$ NMR (400 MHz, Chloroform-d) $\delta 7.68-7.59(\mathrm{~m}, 3 \mathrm{H}), 7.54(\mathrm{dq}, \mathrm{J}=7.5,1.7 \mathrm{~Hz}, 1 \mathrm{H}), 7.51-7.42(\mathrm{~m}, 3 \mathrm{H}), 7.39$ (dd, J = 7.6, 1.7 Hz, 2H), 5.00 (q, J = 6.4 Hz, 1H), 1.58 (d, J = 6.5 Hz, 3H). GC/MS (m/z): 198).
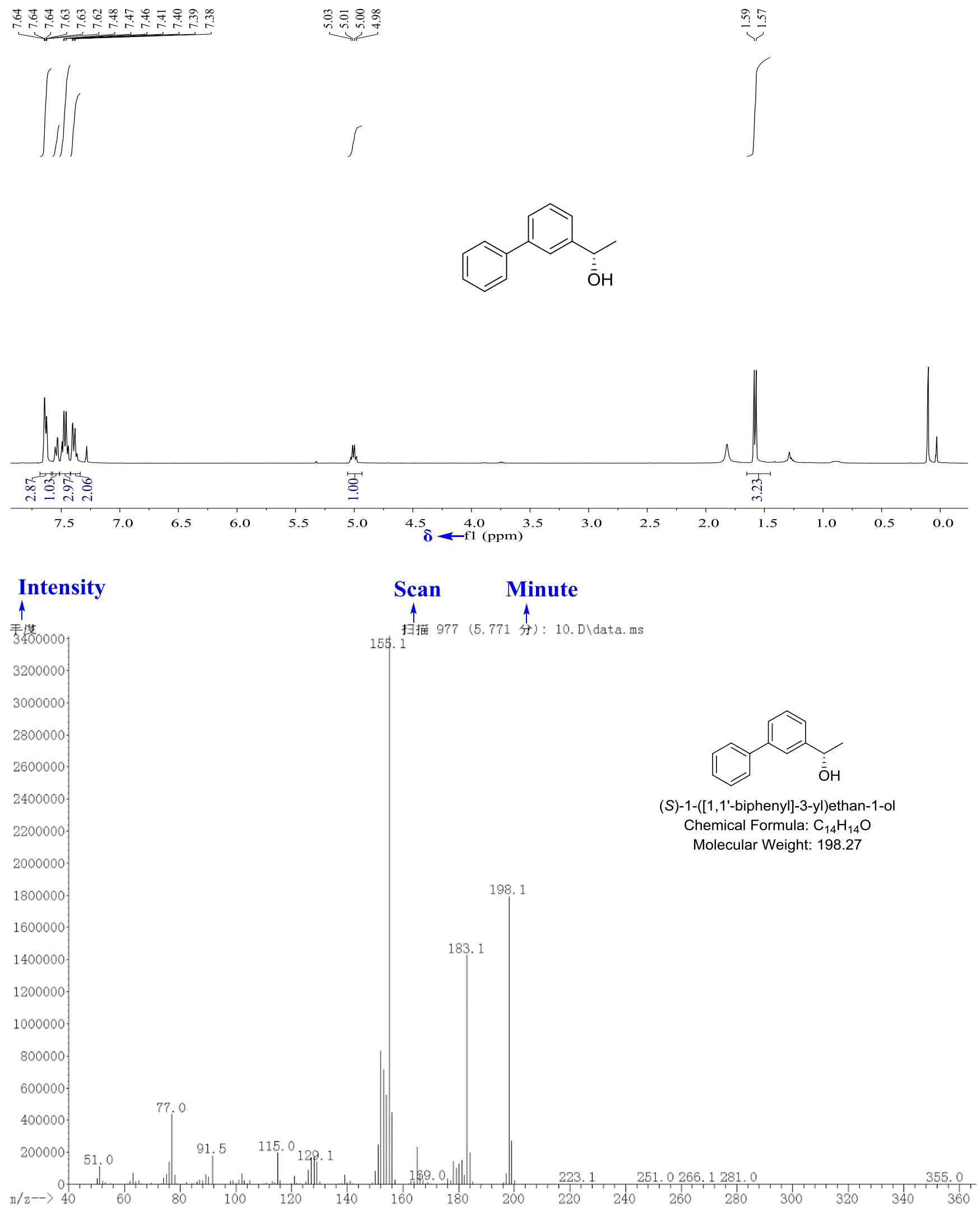
$9 m$ (entry 13 in Table 1): (S)-1-(4'-fluoro-[1,1'-biphenyl]-3-yl)ethan-1-ol $\left({ }^{1} \mathrm{H}\right.$ NMR $(400 \mathrm{MHz}$, Chloroform-d) $\delta 7.63-7.54(\mathrm{~m}, 3 \mathrm{H}), 7.51-7.41(\mathrm{~m}, 2 \mathrm{H}), 7.41-7.36(\mathrm{~m}, 1 \mathrm{H}), 7.15(\mathrm{t}, \mathrm{J}=8.7 \mathrm{~Hz}, 2 \mathrm{H})$, $5.00(\mathrm{q}, \mathrm{J}=6.5 \mathrm{~Hz}, 1 \mathrm{H}), 1.57(\mathrm{~d}, \mathrm{~J}=6.5 \mathrm{~Hz}, 3 \mathrm{H})$. GC/MS (m/z): 216).

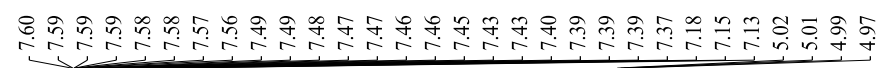
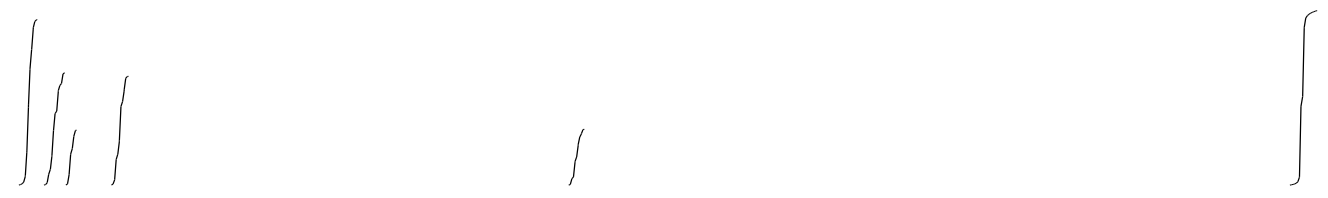<smiles>C[C@H](O)c1cccc(-c2ccc(F)cc2)c1</smiles>
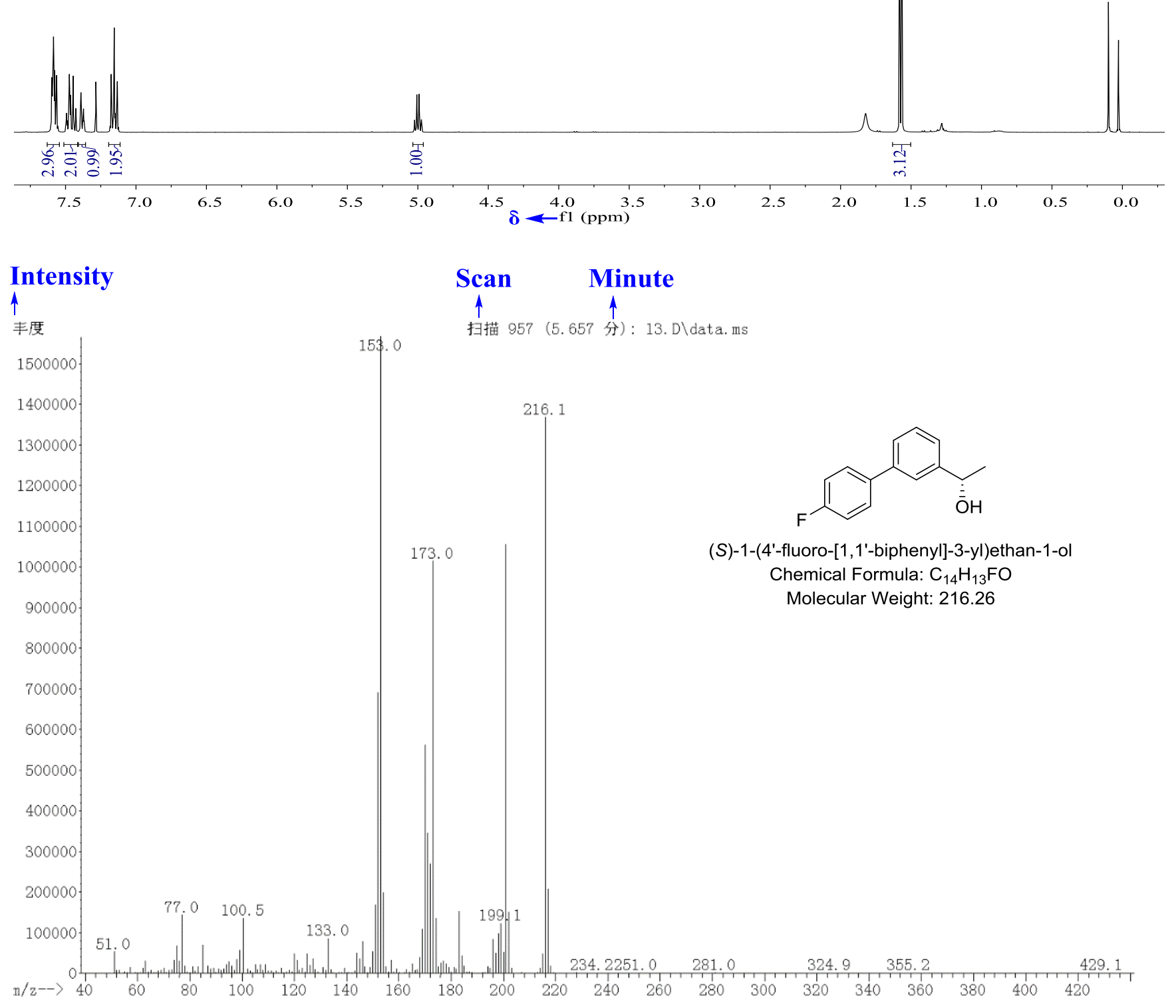
9n (entry 14 in Table 1): (S)-1-(4'-chloro-[1,1'-biphenyl]-3-yl)ethan-1-ol ( ${ }^{1} \mathrm{H}$ NMR (400 MHz, Chloroform-d) $\delta 7.60(\mathrm{~d}, \mathrm{~J}=0.8 \mathrm{~Hz}, 1 \mathrm{H}), 7.58-7.53(\mathrm{~m}, 2 \mathrm{H}), 7.52-7.41(\mathrm{~m}, 4 \mathrm{H}), 7.41-7.37(\mathrm{~m}, 1 \mathrm{H})$, $5.00(\mathrm{q}, \mathrm{J}=6.5 \mathrm{~Hz}, 1 \mathrm{H}), 1.57(\mathrm{~d}, \mathrm{~J}=6.5 \mathrm{~Hz}, 3 \mathrm{H}) . \mathrm{GC} / \mathrm{MS}(\mathrm{m} / \mathrm{z}): 232)$.
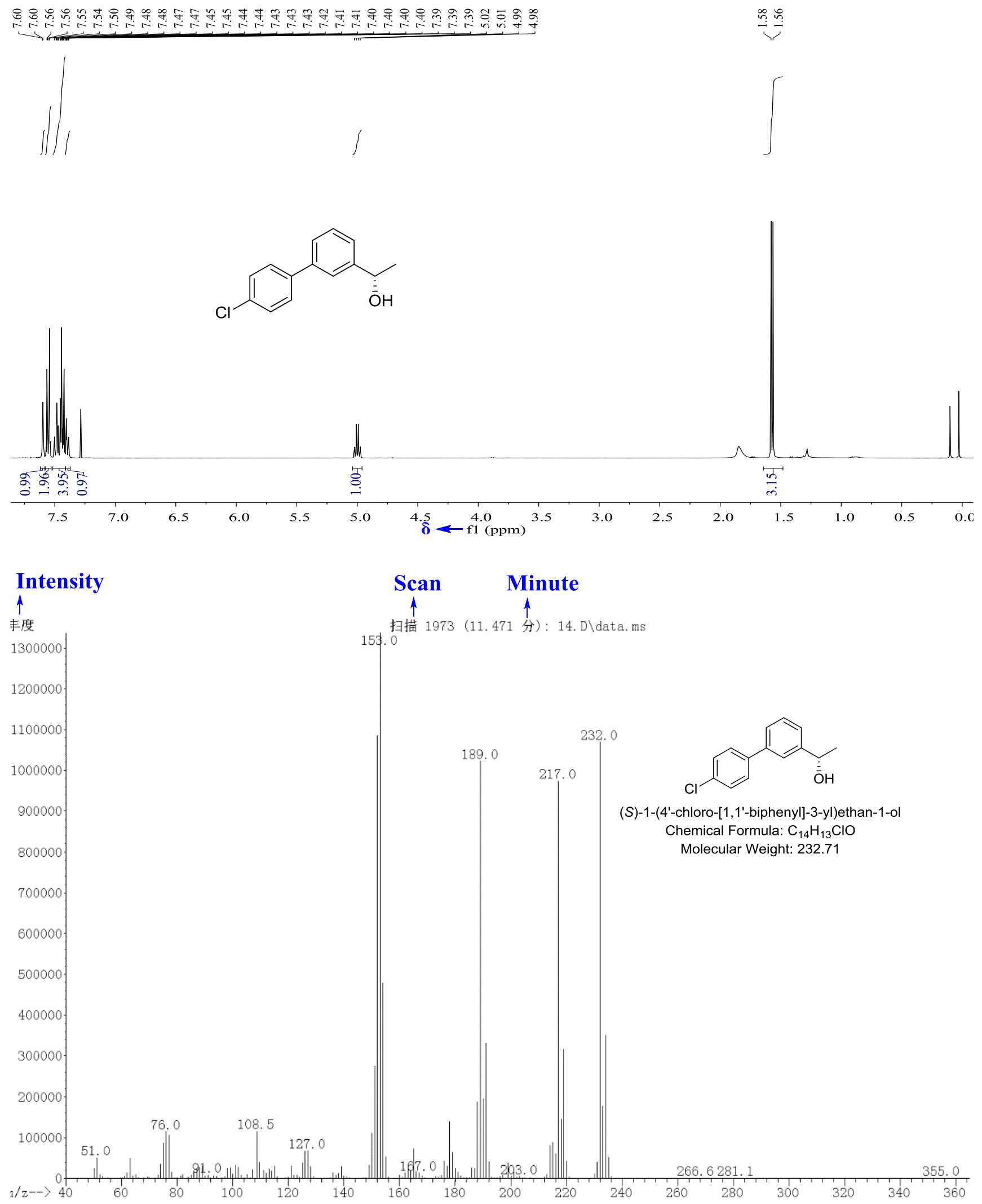
9o (entry 15 in Table 1): (S)-1-(3'-chloro-[1,1'-biphenyl]-3-yl)ethan-1-ol ( ${ }^{1} \mathrm{H}$ NMR (400 MHz, Chloroform-d) $\delta 7.64-7.59(\mathrm{~m}, 2 \mathrm{H}), 7.50(\mathrm{dt}, \mathrm{J}=7.5,1.6 \mathrm{~Hz}, 2 \mathrm{H}), 7.46(\mathrm{td}, \mathrm{J}=7.5,0.6 \mathrm{~Hz}, 1 \mathrm{H}), 7.43-$ $7.37(\mathrm{~m}, 2 \mathrm{H}), 7.35$ (ddd, J = 8.0, 2.0, $1.4 \mathrm{~Hz}, 1 \mathrm{H}), 5.01(\mathrm{q}, \mathrm{J}=6.5 \mathrm{~Hz}, 1 \mathrm{H}), 1.58(\mathrm{~d}, \mathrm{~J}=6.4 \mathrm{~Hz}, 3 \mathrm{H})$. GC/MS (m/z): 232).
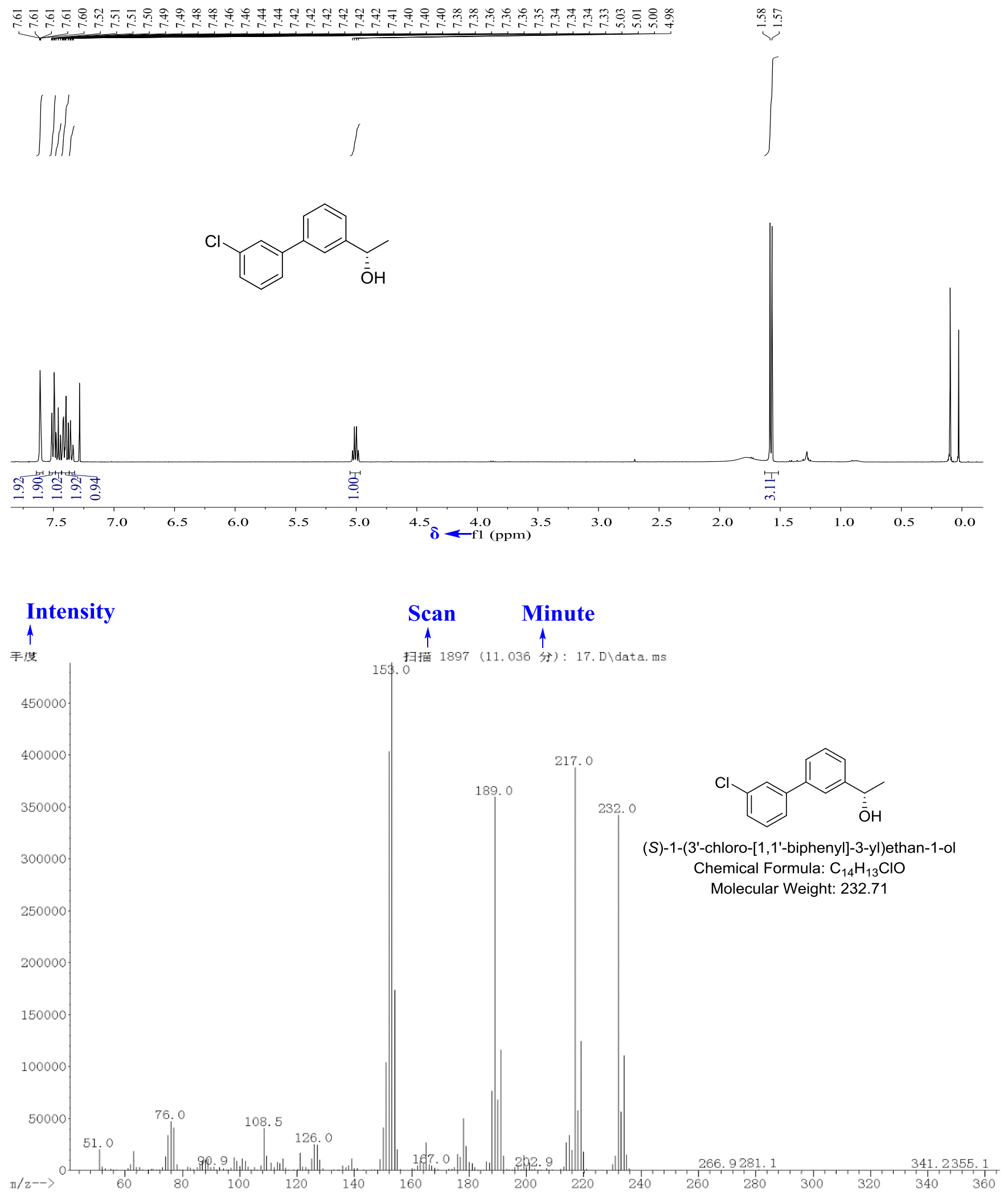
$9 p$ (entry 16 in Table 1): (S)-1-(3'-(trifluoromethyl)-[1,1'-biphenyl]-3-yl)ethan-1-ol $\left({ }^{1} \mathrm{H}\right.$ NMR (400 MHz, Chloroform-d) $\delta 7.86(\mathrm{~s}, 1 \mathrm{H}), 7.80(\mathrm{~d}, \mathrm{~J}=7.4 \mathrm{~Hz}, 1 \mathrm{H}), 7.67-7.56(\mathrm{~m}, 3 \mathrm{H}), 7.54$ (dt, J = 7.5, $1.7 \mathrm{~Hz}, 1 \mathrm{H}), 7.49(\mathrm{td}, \mathrm{J}=7.5,0.6 \mathrm{~Hz}, 1 \mathrm{H}), 7.46-7.41(\mathrm{~m}, 1 \mathrm{H}), 5.03(\mathrm{q}, J=6.4 \mathrm{~Hz}, 1 \mathrm{H}), 1.59(\mathrm{~d}, J=6.5$ $\mathrm{Hz}, 3 \mathrm{H})$. GC/MS (m/z): 266).

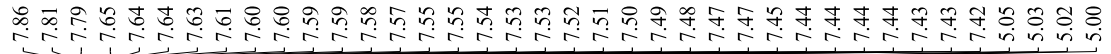
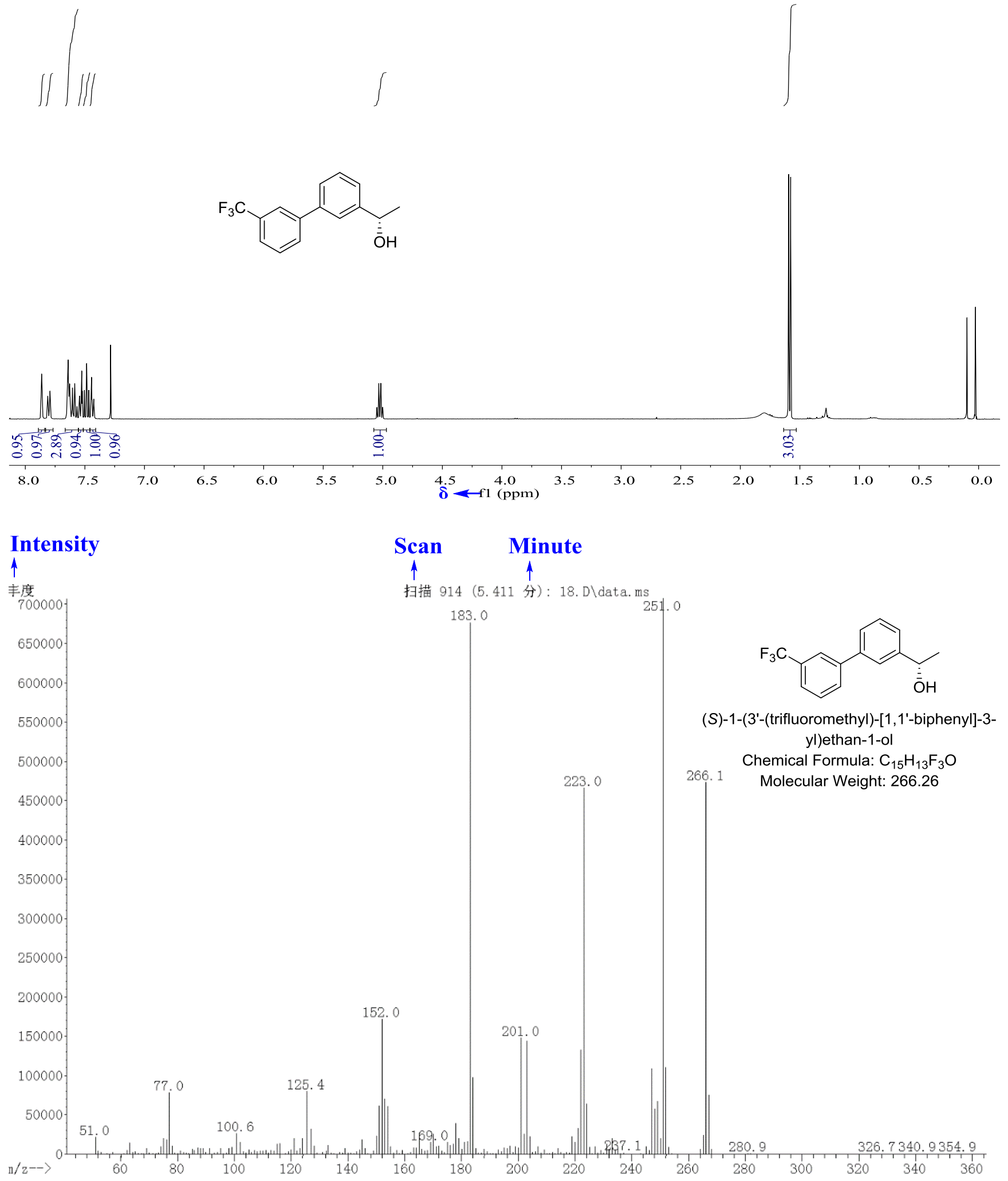
9q (entry 17 in Table 1): (S)-1-(4'-methyl-[1,1'-biphenyl]-3-yl)ethan-1-ol $\left({ }^{1} \mathrm{H}\right.$ NMR (400 MHz, Chloroform-d) $\delta 7.62$ (d, J = 1.9 Hz, 1H), 7.53 (dd, J = 7.0, $4.8 \mathrm{~Hz}, 3 \mathrm{H}), 7.44(\mathrm{~s}, 1 \mathrm{H}), 7.38(\mathrm{~s}, 1 \mathrm{H}), 7.29$ $(\mathrm{d}, \mathrm{J}=7.7 \mathrm{~Hz}, 2 \mathrm{H}), 4.99(\mathrm{q}, \mathrm{J}=6.5 \mathrm{~Hz}, 1 \mathrm{H}), 2.43(\mathrm{~s}, 3 \mathrm{H}), 1.58(\mathrm{~d}, \mathrm{~J}=6.5 \mathrm{~Hz}, 3 \mathrm{H}) . \mathrm{GC} / \mathrm{MS}(\mathrm{m} / \mathrm{z}): 212)$.
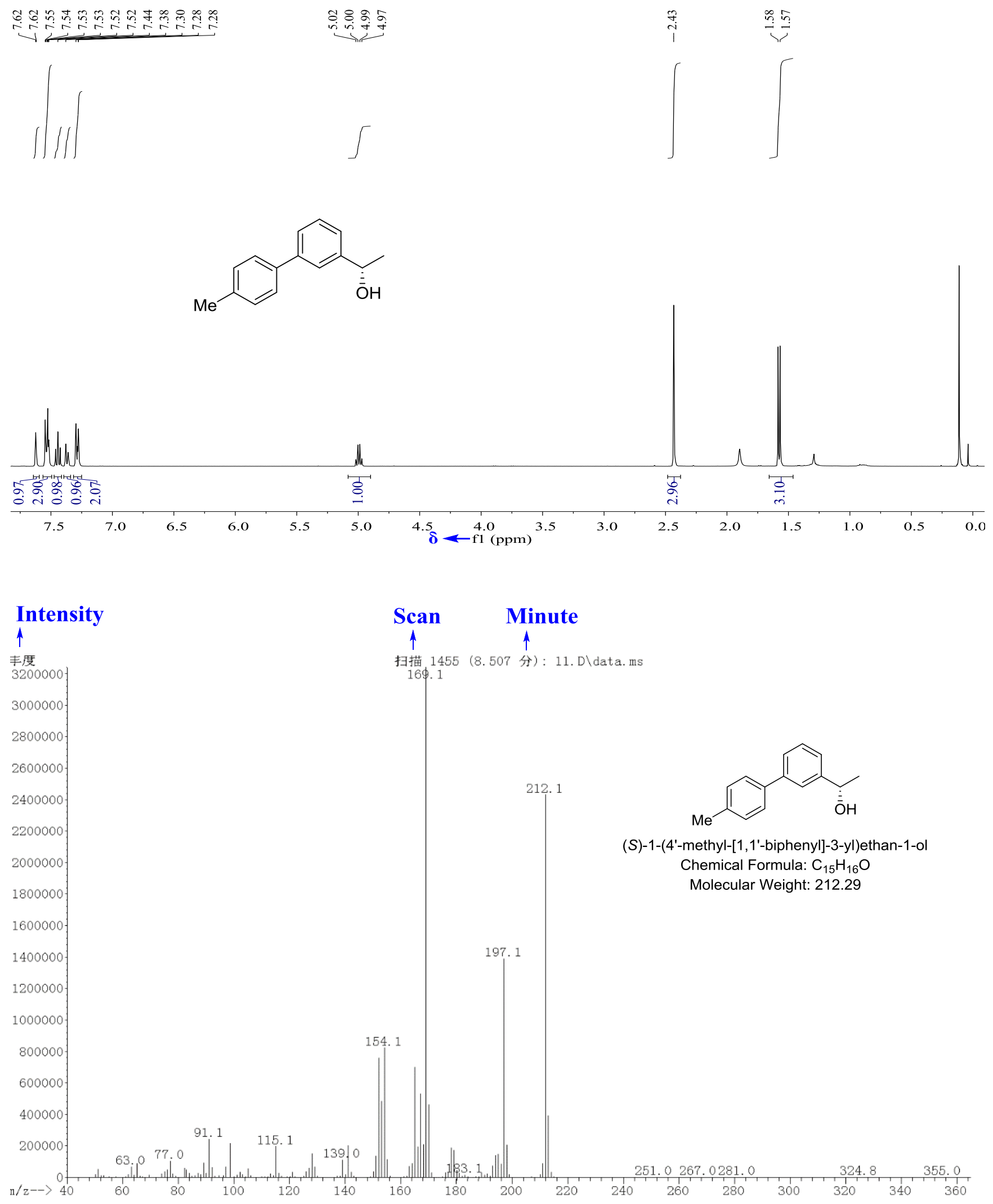
9r (entry 18 in Table 1): (S)-1-(3'-methyl-[1,1'-biphenyl]-3-yl)ethan-1-ol $\left({ }^{1} \mathrm{H}\right.$ NMR (400 MHz, Chloroform-d) $\delta 7.63(\mathrm{t}, \mathrm{J}=1.8 \mathrm{~Hz}, 1 \mathrm{H}), 7.53(\mathrm{dt}, \mathrm{J}=7.6,1.6 \mathrm{~Hz}, 1 \mathrm{H}), 7.48-7.40(\mathrm{~m}, 3 \mathrm{H}), 7.40-7.32$ $(\mathrm{m}, 2 \mathrm{H}), 7.23-7.16(\mathrm{~m}, 1 \mathrm{H}), 5.00(\mathrm{q}, \mathrm{J}=6.4 \mathrm{~Hz}, 1 \mathrm{H}), 2.45(\mathrm{~s}, 3 \mathrm{H}), 1.58(\mathrm{~d}, \mathrm{~J}=6.5 \mathrm{~Hz}, 3 \mathrm{H}) . \mathrm{GC} / \mathrm{MS}$ $(\mathrm{m} / \mathrm{z}): 212)$.
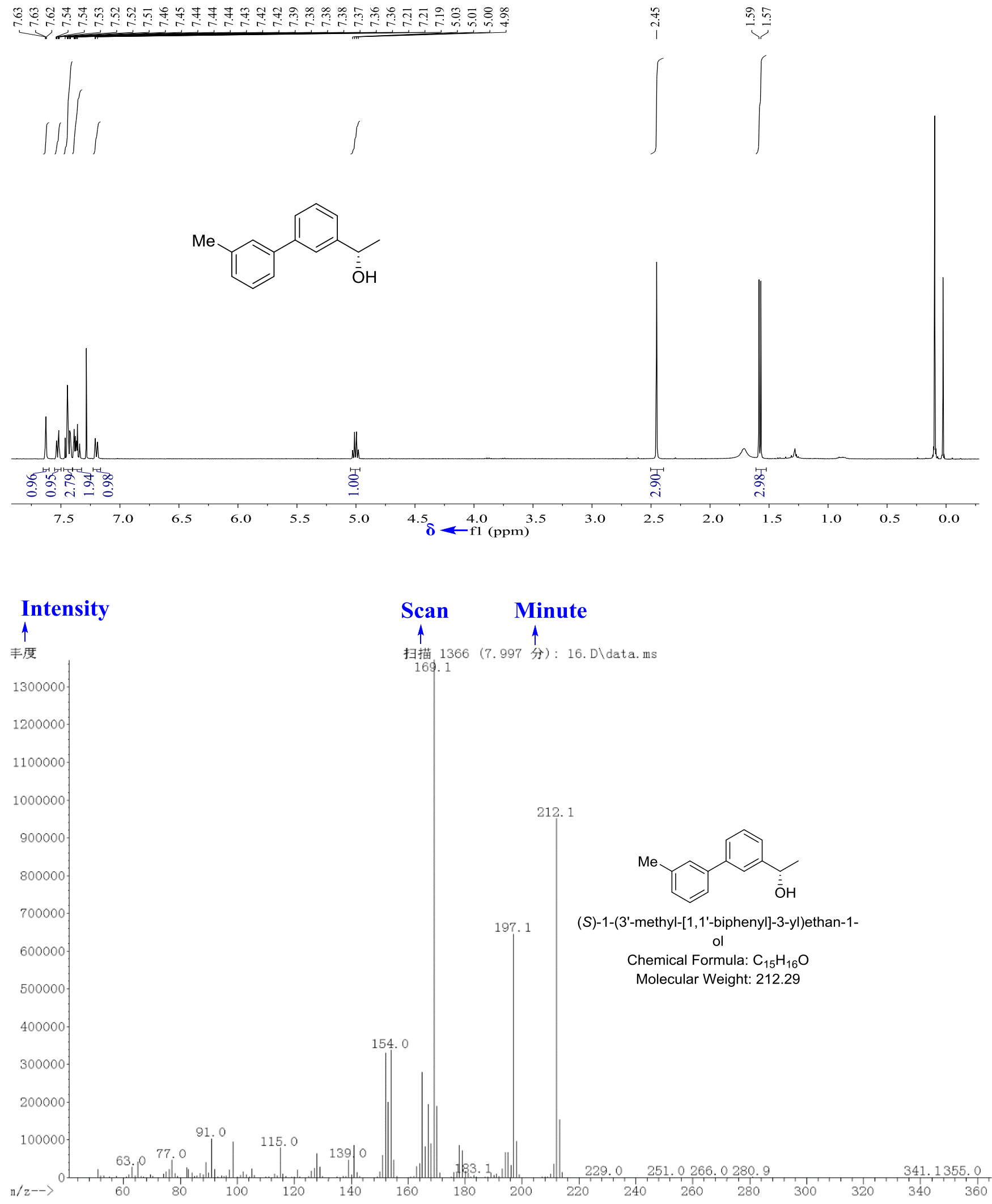
9s (entry 19 in Table 1): (S)-1-(4'-methoxy-[1,1'-biphenyl]-3-yl)ethan-1-ol ( ${ }^{1} \mathrm{H}$ NMR (400 MHz, Chloroform-d) $\delta 7.62-7.53(\mathrm{~m}, 3 \mathrm{H}), 7.52-7.47(\mathrm{~m}, 1 \mathrm{H}), 7.46-7.39(\mathrm{~m}, 1 \mathrm{H}), 7.38-7.31(\mathrm{~m}, 1 \mathrm{H})$, $7.01(\mathrm{~d}, \mathrm{~J}=8.8 \mathrm{~Hz}, 2 \mathrm{H}), 4.99(\mathrm{q}, \mathrm{J}=6.5 \mathrm{~Hz}, 1 \mathrm{H}), 3.88(\mathrm{~s}, 3 \mathrm{H}), 1.57$ (d, J = 6.5 Hz, 3H). GC/MS (m/z): 228).
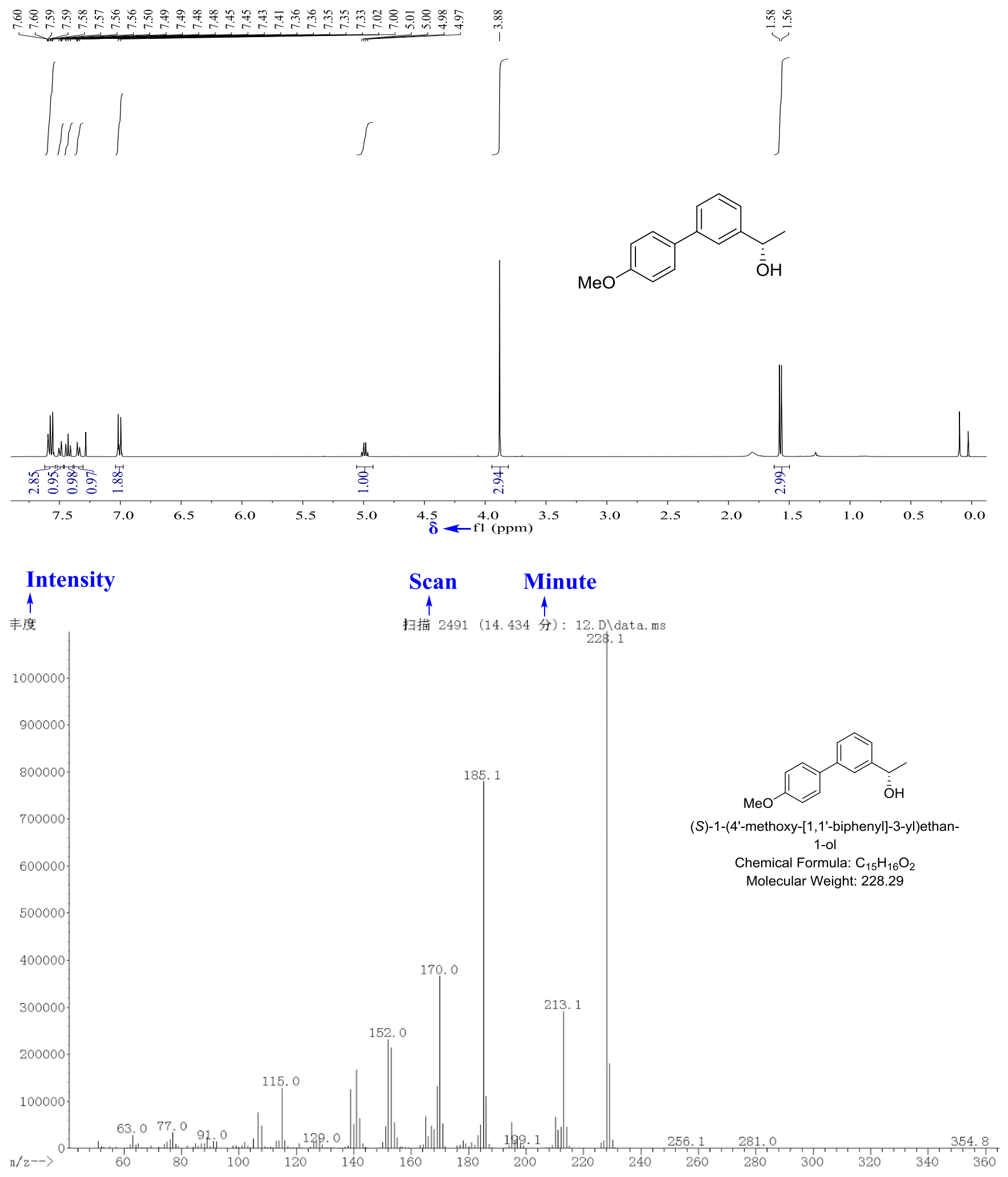
9t (entry 20 in Table 1): (S)-1-(3-(thiophen-3-yl)phenyl)ethanol ( ${ }^{1} \mathrm{H}$ NMR (400 MHz, Chloroform-

d) $\delta 7.55(\mathrm{~s}, 1 \mathrm{H}), 7.44(\mathrm{~d}, \mathrm{~J}=7.7 \mathrm{~Hz}, 1 \mathrm{H}), 7.42-7.39(\mathrm{~m}, 1 \mathrm{H}), 7.36-7.29(\mathrm{~m}, 3 \mathrm{H}), 7.23$ (d, J = 7.6 Hz, 1H), 4.89 (q, J = 6.4 Hz, 1H), 1.47 (d, J = 6.5 Hz, 3H). GC/MS (m/z): 204).
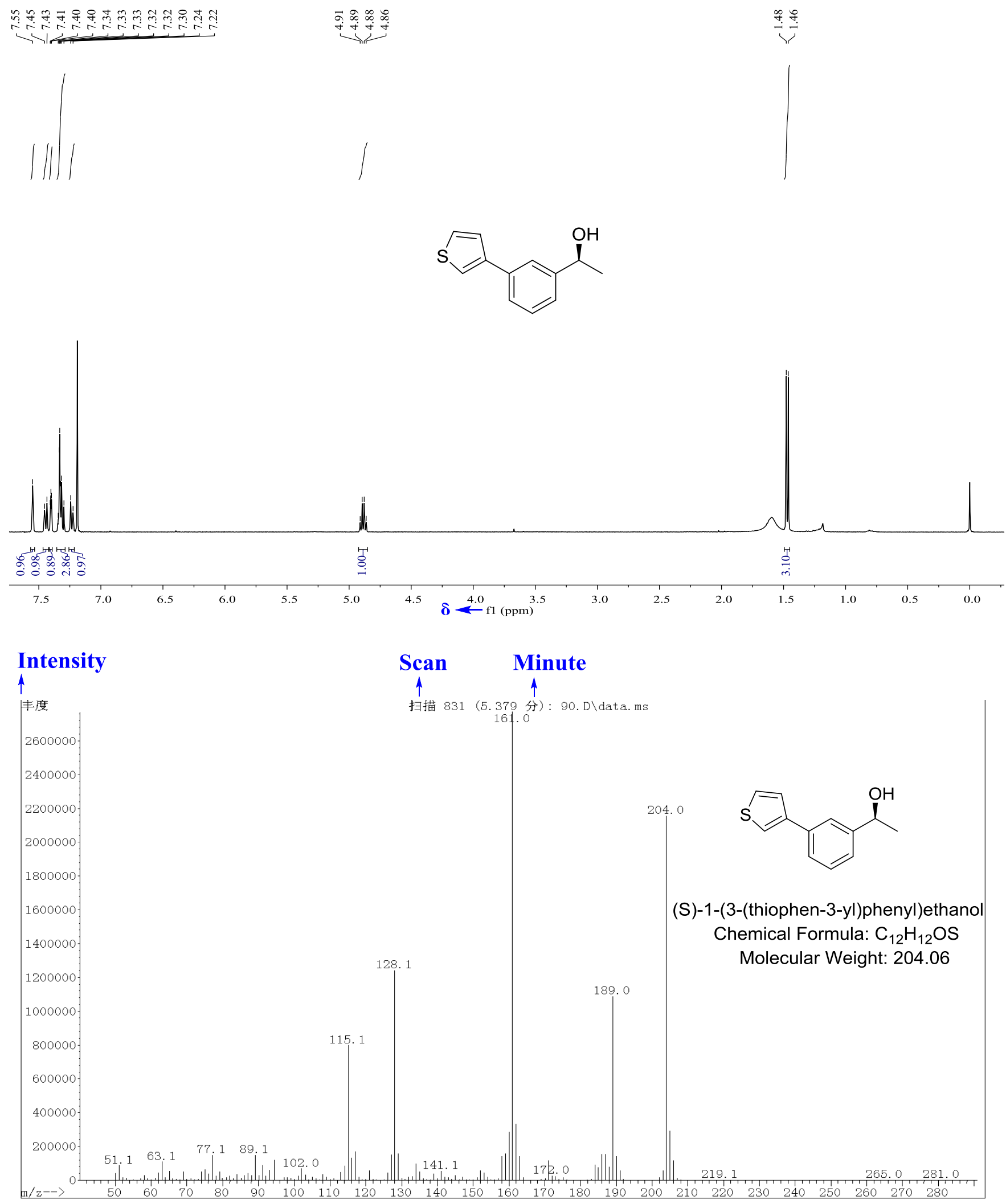
9u (entry 21 in Table 1): (S)-1-(3-(pyridin-4-yl)phenyl)ethanol ( ${ }^{1} \mathrm{H}$ NMR (400 MHz, Chloroform-d) $\delta 8.59(\mathrm{~d}, \mathrm{~J}=5.9 \mathrm{~Hz}, 2 \mathrm{H}), 7.62-7.51(\mathrm{~m}, 4 \mathrm{H}), 7.46(\mathrm{~d}, \mathrm{~J}=8.2 \mathrm{~Hz}, 2 \mathrm{H}), 4.92(\mathrm{q}, \mathrm{J}=6.5 \mathrm{~Hz}, 1 \mathrm{H}), 1.48$ $(\mathrm{d}, \mathrm{J}=6.5 \mathrm{~Hz}, 3 \mathrm{H}) . \mathrm{GC} / \mathrm{MS}(\mathrm{m} / \mathrm{z})$ : 199).
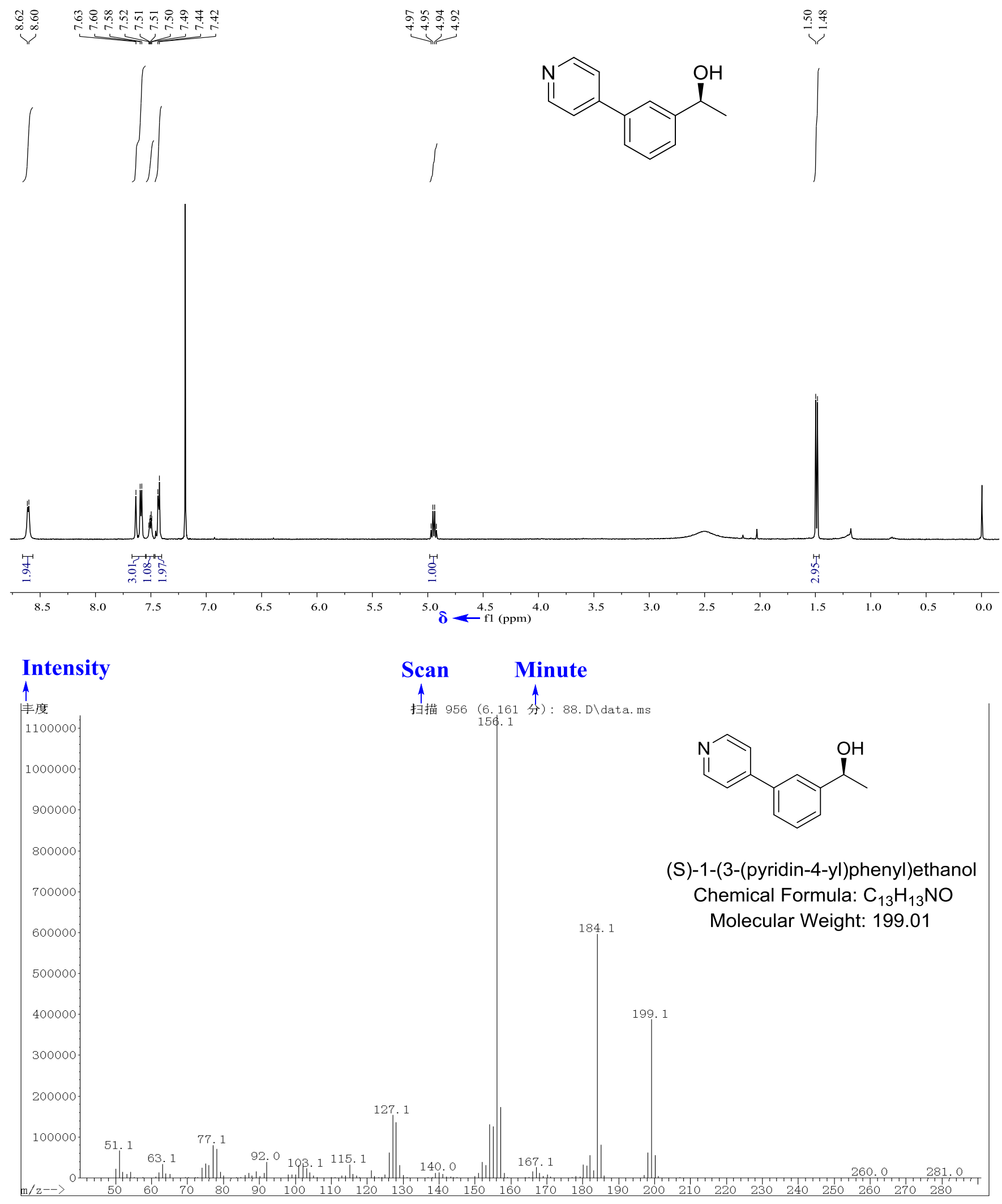
$12 a$ (entry 1 in Table 2): (S)-1-phenyl-3-(phenylamino)propan-1-ol $\left({ }^{1} \mathrm{H}\right.$ NMR (400 MHz, Chloroform-d) $\delta 7.33-7.20(\mathrm{~m}, 5 \mathrm{H}), 7.15-7.09(\mathrm{~m}, 2 \mathrm{H}), 6.69(\mathrm{t}, \mathrm{J}=7.3 \mathrm{~Hz}, 1 \mathrm{H}), 6.61(\mathrm{~d}, \mathrm{~J}=7.7 \mathrm{~Hz}$, 2H), 4.85 (dd, J = 7.6, $5.1 \mathrm{~Hz}, 1 \mathrm{H}), 3.24$ (t, J = 6.4 Hz, 2H), $2.05-1.96$ (m, 2H). GC/MS (m/z): 227).
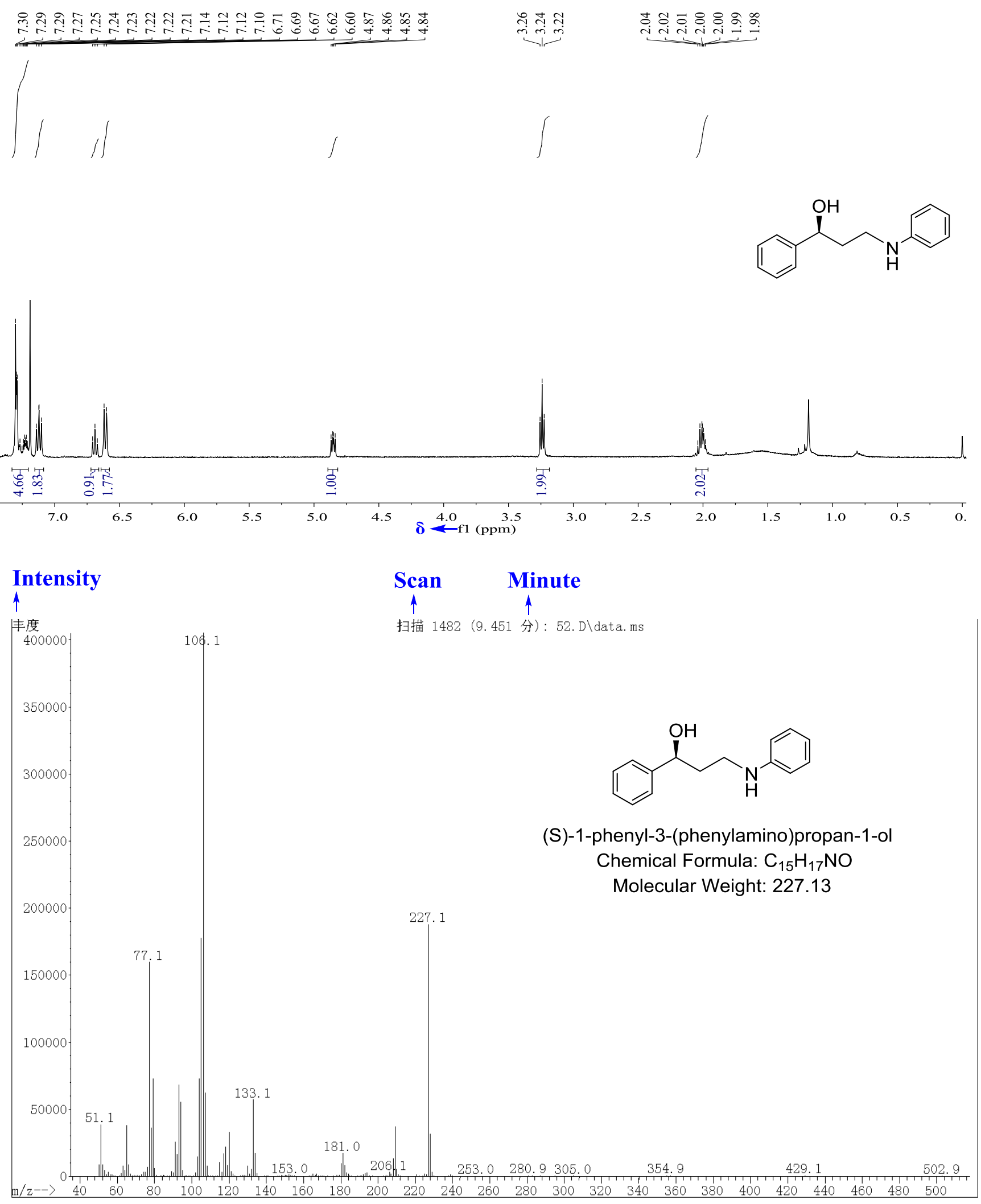


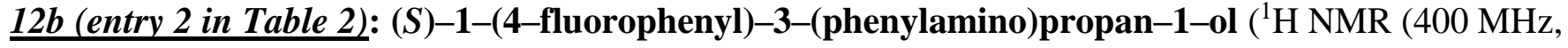
Chloroform-d) $\delta 7.32(\mathrm{dd}, \mathrm{J}=8.5,5.4 \mathrm{~Hz}, 2 \mathrm{H}), 7.25(\mathrm{~d}, \mathrm{~J}=5.0 \mathrm{~Hz}, 4 \mathrm{H}), 7.01(\mathrm{t}, \mathrm{J}=8.7 \mathrm{~Hz}, 2 \mathrm{H}), 6.91$ $(\mathrm{dd}, \mathrm{J}=11.8,7.7 \mathrm{~Hz}, 3 \mathrm{H}), 4.95(\mathrm{t}, \mathrm{J}=6.4 \mathrm{~Hz}, 1 \mathrm{H}), 3.41-3.29(\mathrm{~m}, 2 \mathrm{H}), 2.08(\mathrm{q}, \mathrm{J}=6.2 \mathrm{~Hz}, 2 \mathrm{H})$. GC/MS (m/z): 245).
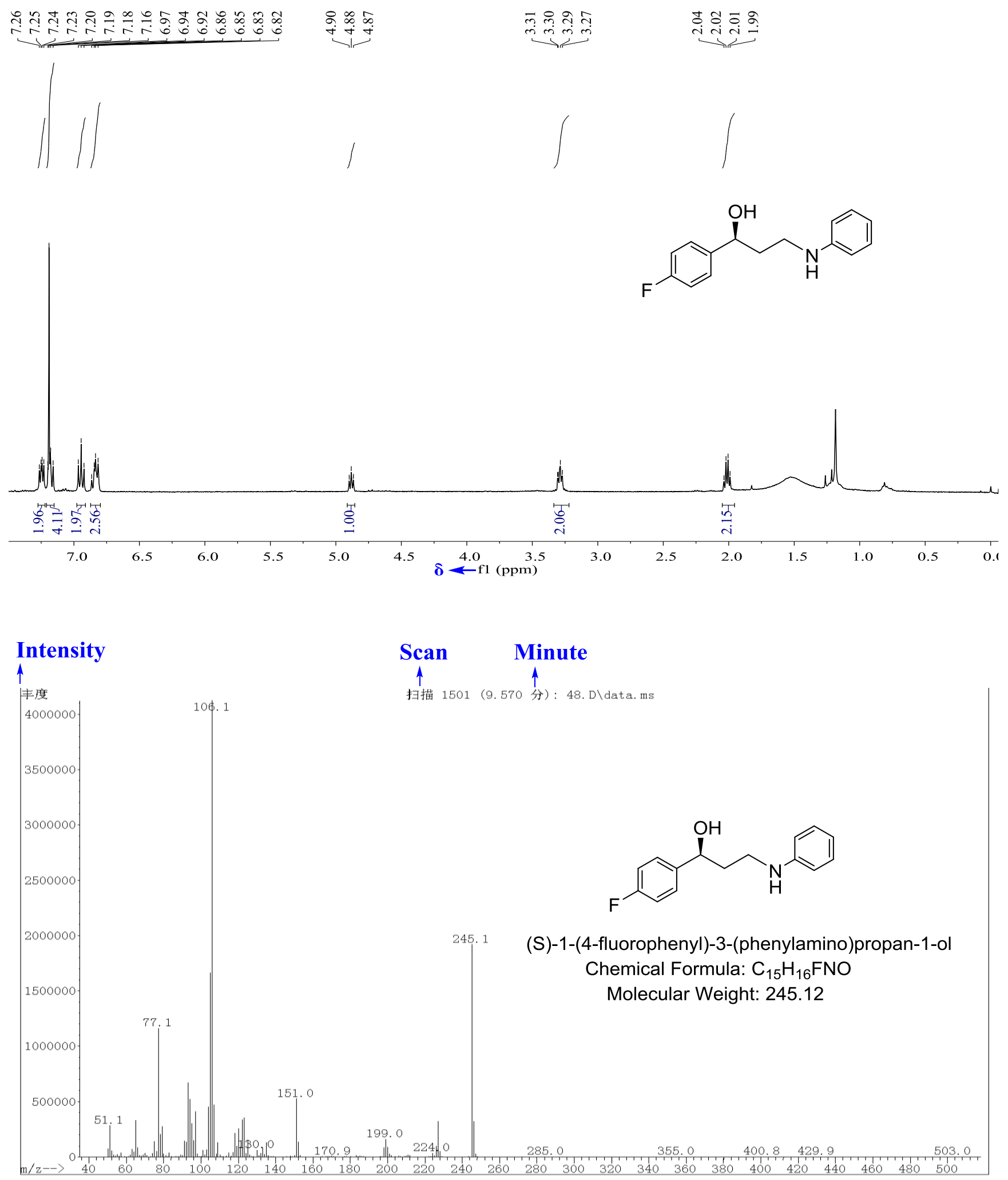
12c (entry 3 in Table 2): (S)-1-(4-chlorophenyl)-3-(phenylamino)propan-1-ol ( ${ }^{1} \mathrm{H}$ NMR (400 MHz, Chloroform-d) $\delta 7.34-7.27(\mathrm{~m}, 4 \mathrm{H}), 7.21(\mathrm{td}, \mathrm{J}=7.4,1.8 \mathrm{~Hz}, 2 \mathrm{H}), 6.81(\mathrm{tt}, \mathrm{J}=7.3,1.1 \mathrm{~Hz}, 1 \mathrm{H}), 6.77-$ $6.71(\mathrm{~m}, 2 \mathrm{H}), 4.92(\mathrm{t}, \mathrm{J}=6.3 \mathrm{~Hz}, 1 \mathrm{H}), 3.32(\mathrm{td}, \mathrm{J}=6.2,2.0 \mathrm{~Hz}, 2 \mathrm{H}), 2.04(\mathrm{q}, \mathrm{J}=6.3 \mathrm{~Hz}, 2 \mathrm{H}) . \mathrm{GC} / \mathrm{MS}$ (m/z): 261).
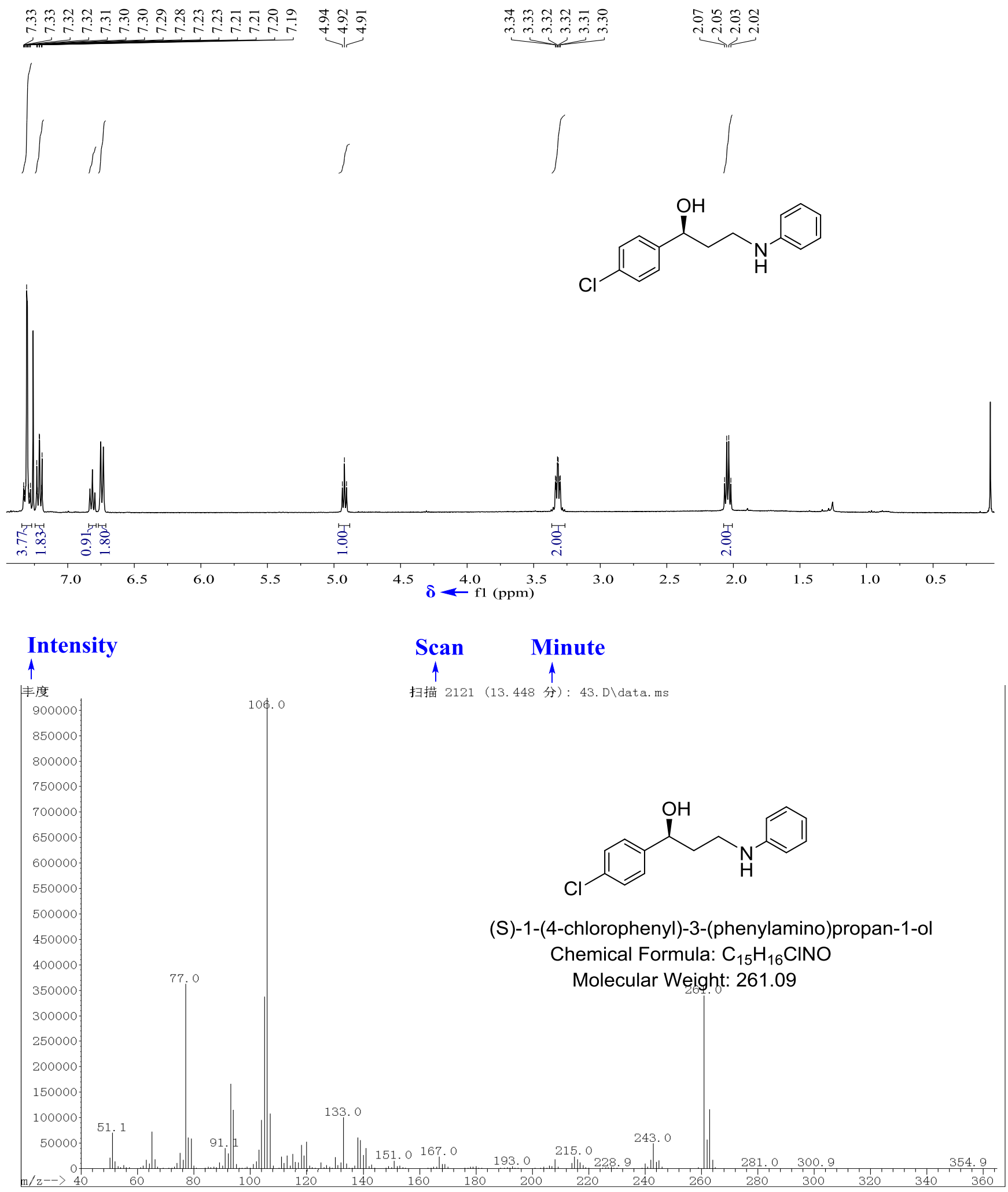
12d (entry 4 in Table 2): (S)-1-(4-bromophenyl)-3-(phenylamino)propan-1-ol ( ${ }^{1} \mathrm{H}$ NMR (400 MHz, Chloroform-d) $\delta 7.39(\mathrm{~d}, \mathrm{~J}=8.3 \mathrm{~Hz}, 2 \mathrm{H}), 7.18-7.11(\mathrm{~m}, 4 \mathrm{H}), 6.75(\mathrm{t}, \mathrm{J}=7.3 \mathrm{~Hz}, 1 \mathrm{H}), 6.68(\mathrm{~d}, \mathrm{~J}$ $=8.1 \mathrm{~Hz}, 2 \mathrm{H}), 4.83(\mathrm{t}, \mathrm{J}=6.3 \mathrm{~Hz}, 1 \mathrm{H}), 3.25(\mathrm{td}, \mathrm{J}=6.3,2.1 \mathrm{~Hz}, 2 \mathrm{H}), 1.97(\mathrm{q}, \mathrm{J}=6.3 \mathrm{~Hz}, 2 \mathrm{H})$. GC/MS $(\mathrm{m} / \mathrm{z}): 305)$.
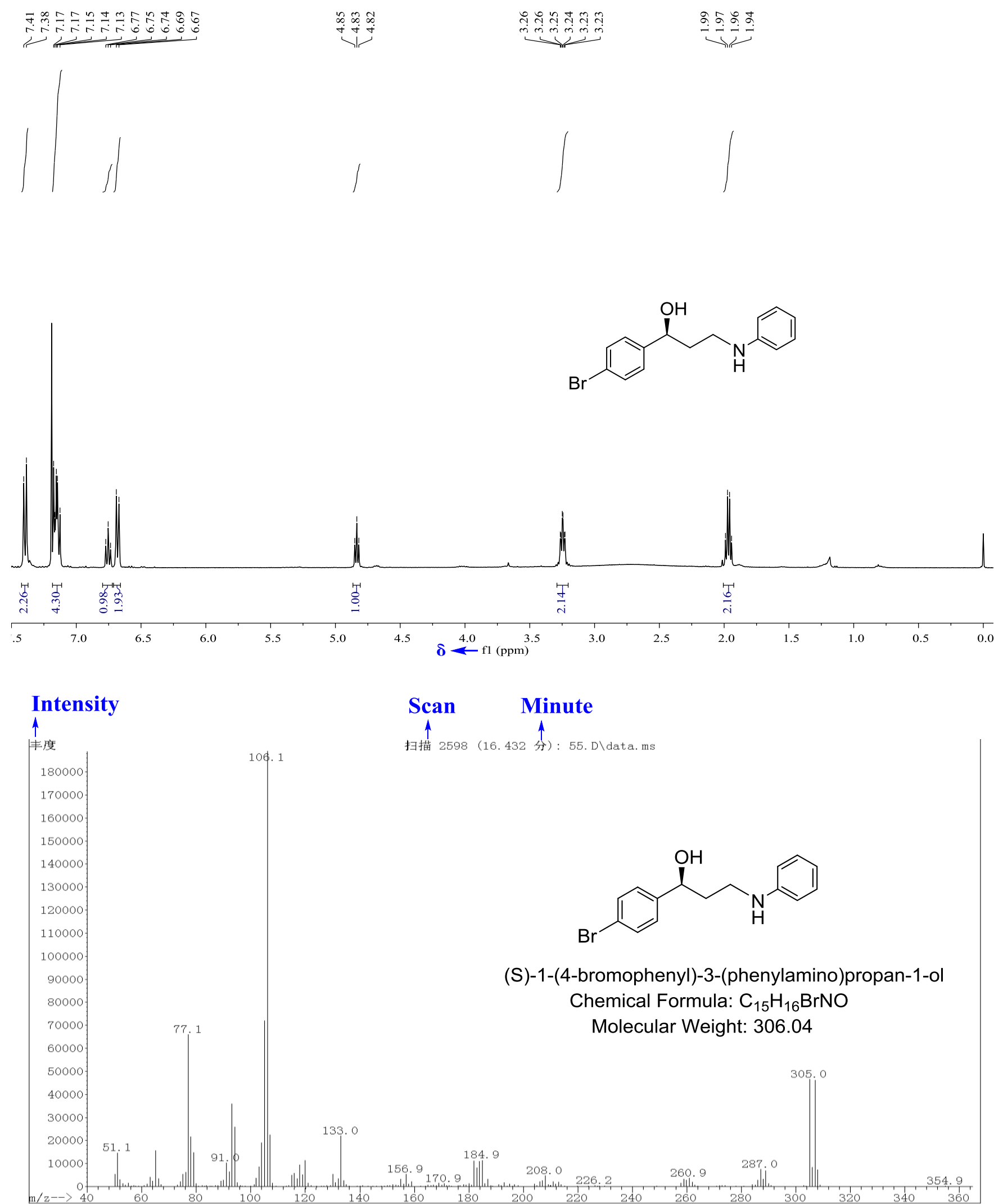
12e (entry 5 in Table 2): (S)-1-(4-iodophenyl)-3-(phenylamino)propan-1-ol ( ${ }^{1} \mathrm{H}$ NMR (400 MHz, Chloroform-d) $\delta 7.67(\mathrm{~d}, \mathrm{~J}=8.0 \mathrm{~Hz}, 2 \mathrm{H}), 7.24-7.06(\mathrm{~m}, 4 \mathrm{H}), 6.76(\mathrm{t}, \mathrm{J}=7.1 \mathrm{~Hz}, 1 \mathrm{H}), 6.65(\mathrm{~d}, \mathrm{~J}=7.9$ $\mathrm{Hz}, 2 \mathrm{H}), 4.86(\mathrm{t}, \mathrm{J}=6.1 \mathrm{~Hz}, 1 \mathrm{H}), 3.28(\mathrm{t}, \mathrm{J}=5.7 \mathrm{~Hz}, 2 \mathrm{H}), 2.01(\mathrm{q}, \mathrm{J}=6.1 \mathrm{~Hz}, 2 \mathrm{H}) . \mathrm{GC} / \mathrm{MS}(\mathrm{m} / \mathrm{z}): 353)$.
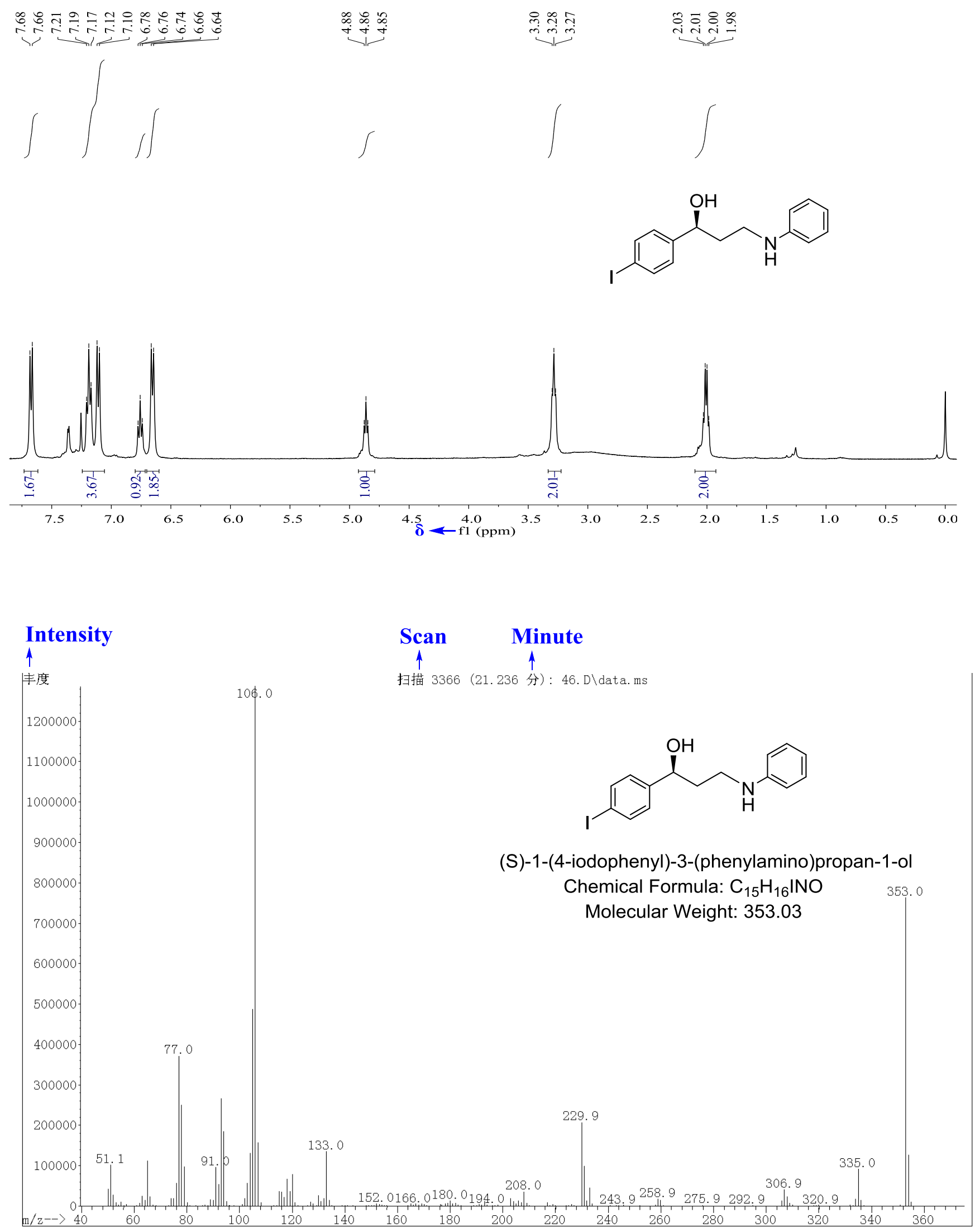
$12 f$ (entry 6 in Table 2): (S)-4-(1-hydroxy-3-(phenylamino)propyl)benzonitrile $\left({ }^{1} \mathrm{H}\right.$ NMR (400 MHz, Chloroform-d) $\delta 7.62(\mathrm{~d}, \mathrm{~J}=8.2 \mathrm{~Hz}, 2 \mathrm{H}), 7.47(\mathrm{~d}, \mathrm{~J}=8.1 \mathrm{~Hz}, 2 \mathrm{H}), 7.20$ (t, J = 7.6 Hz, 2H), 6.79 (t, $\mathrm{J}=7.0 \mathrm{~Hz}, 1 \mathrm{H}), 6.68(\mathrm{~d}, \mathrm{~J}=8.4 \mathrm{~Hz}, 2 \mathrm{H}), 4.98(\mathrm{dd}, \mathrm{J}=8.1,4.3 \mathrm{~Hz}, 1 \mathrm{H}), 3.33(\mathrm{td}, \mathrm{J}=6.2,2.3 \mathrm{~Hz}, 2 \mathrm{H})$, $2.08-1.94(\mathrm{~m}, 2 \mathrm{H}) . \mathrm{GC} / \mathrm{MS}(\mathrm{m} / \mathrm{z}): 252)$.
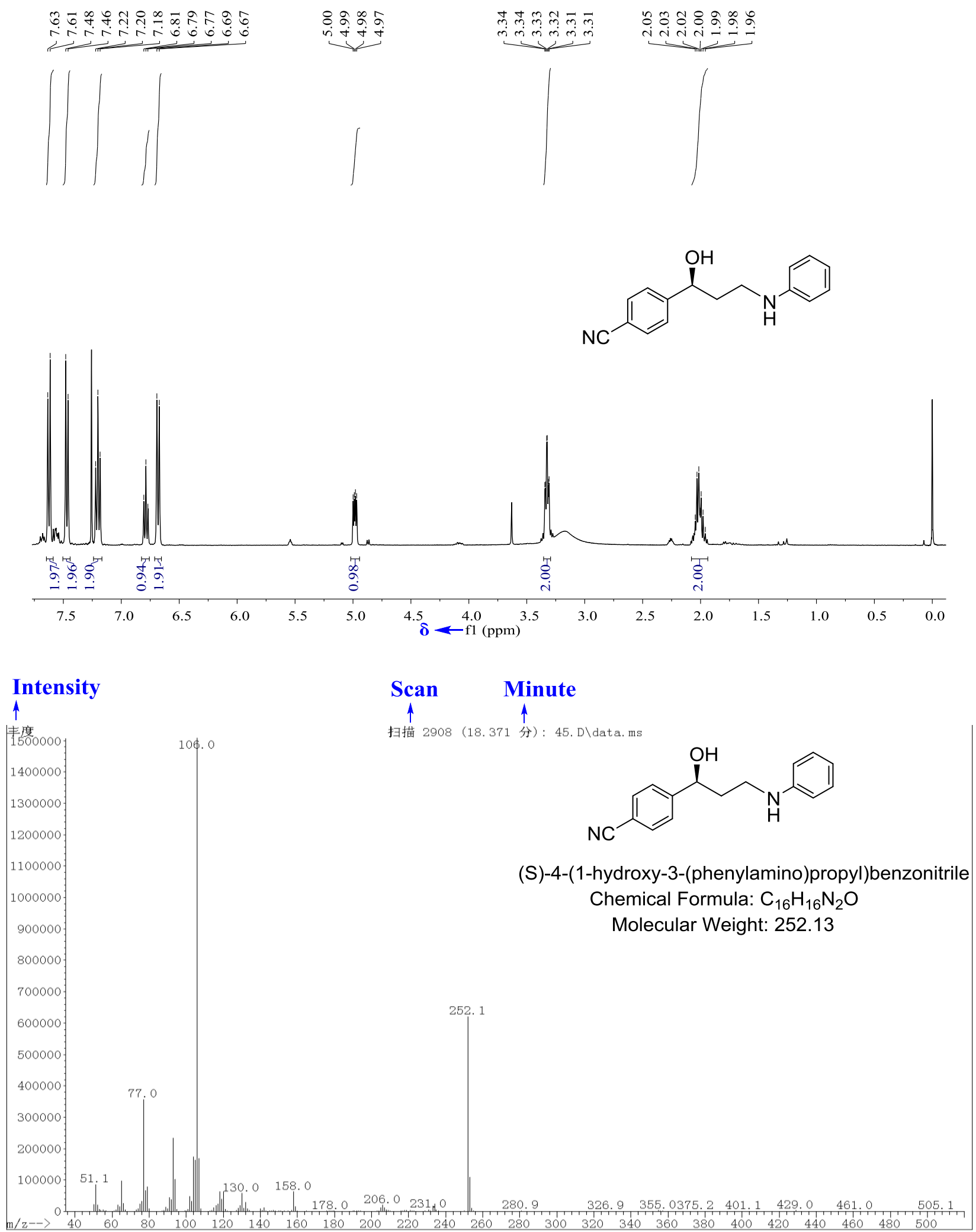
$12 \mathrm{~g}$ (entry 7 in Table 2): (S)-3-(phenylamino)-1-(p-tolyl)propan-1-ol $\left({ }^{1} \mathrm{H}\right.$ NMR (400 MHz, Chloroform-d) $\delta 7.17$ (d, J = 12.1 Hz, 5H), $7.08(\mathrm{~d}, \mathrm{~J}=8.1 \mathrm{~Hz}, 2 \mathrm{H}), 6.89$ (d, J = 7.0 Hz, 2H), 4.86 (dd, J = 7.9, $4.8 \mathrm{~Hz}, 1 \mathrm{H}), 3.30(\mathrm{t}, \mathrm{J}=6.3 \mathrm{~Hz}, 2 \mathrm{H}), 2.27$ (s, 3H), 2.05 (q, J = 6.4 Hz, 2H). GC/MS (m/z): 241).
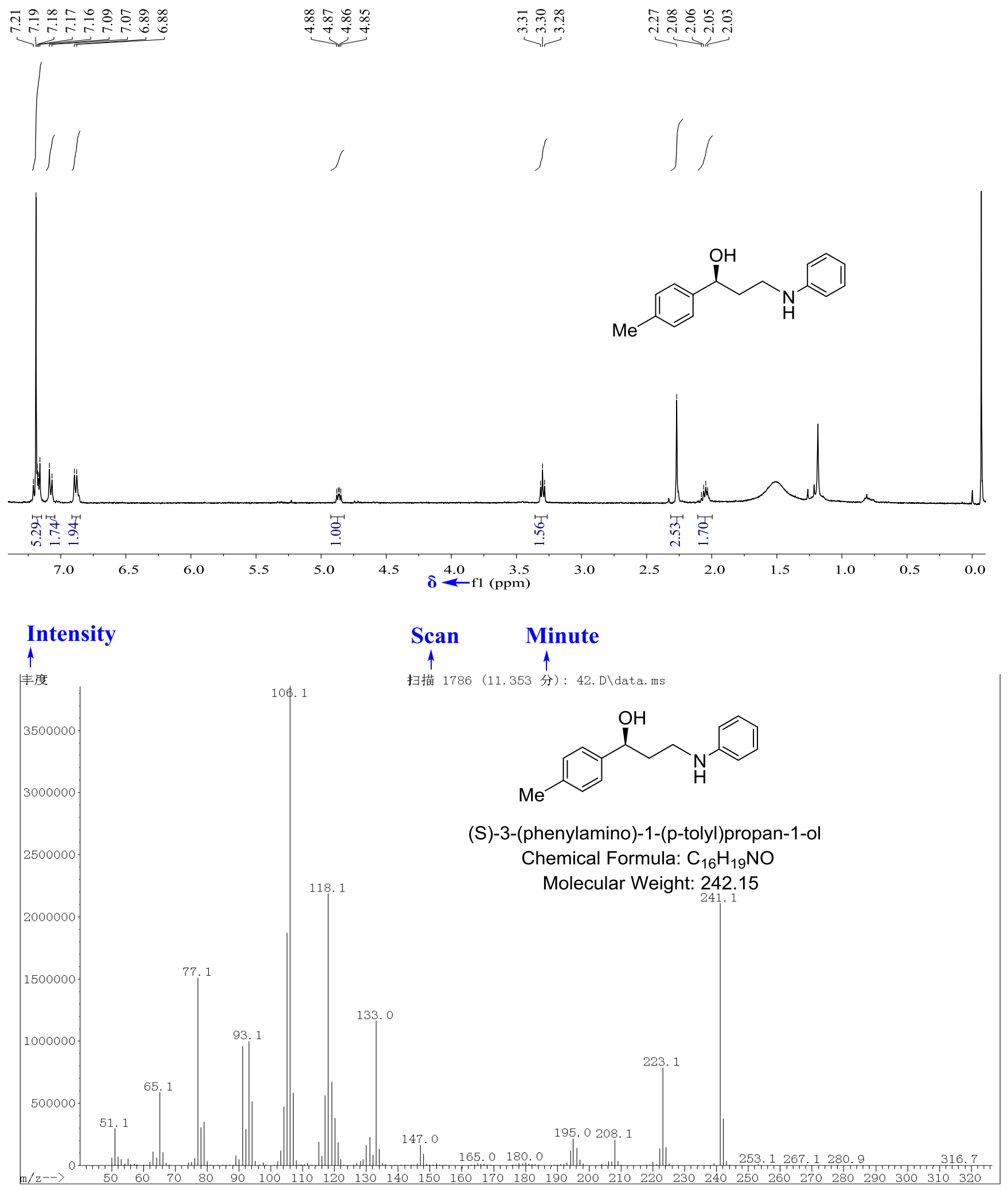
12h (entry 8 in Table 2): (S)-1-(4-methoxyphenyl)-3-(phenylamino)propan-1-ol ( ${ }^{1} \mathrm{H}$ NMR (400 MHz, Chloroform-d) $\delta 7.31(\mathrm{~d}, \mathrm{~J}=8.6 \mathrm{~Hz}, 2 \mathrm{H}), 7.21(\mathrm{t}, \mathrm{J}=7.8 \mathrm{~Hz}, 2 \mathrm{H}), 6.91(\mathrm{~d}, \mathrm{~J}=8.6 \mathrm{~Hz}, 2 \mathrm{H}), 6.78$ (t, $\mathrm{J}=7.2 \mathrm{~Hz}, 1 \mathrm{H}), 6.68(\mathrm{~d}, \mathrm{~J}=8.1 \mathrm{~Hz}, 2 \mathrm{H}), 4.87(\mathrm{dd}, \mathrm{J}=7.9,4.8 \mathrm{~Hz}, 1 \mathrm{H}), 3.83(\mathrm{~s}, 3 \mathrm{H}), 3.29$ (t, J = 6.4 Hz, $2 \mathrm{H}), 2.13-2.01(\mathrm{~m}, 2 \mathrm{H})$. GC/MS (m/z): 257).
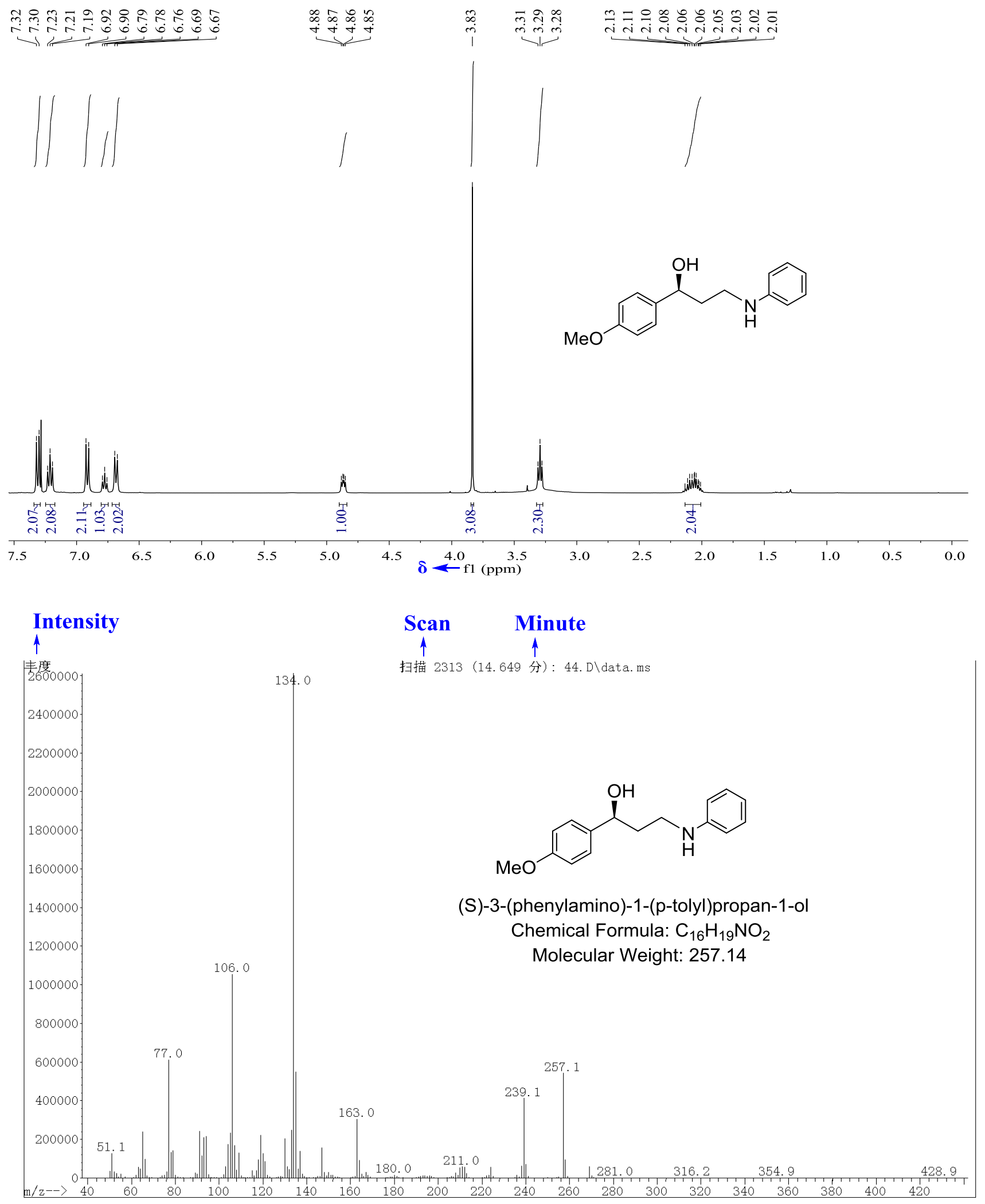
12i (entry 9 in Table 2): (S)-3-((4-chlorophenyl)amino)-1-phenylpropan-1-ol ( ${ }^{1} \mathrm{H}$ NMR (400 MHz, Chloroform-d) $\delta 7.39-7.27(\mathrm{~m}, 5 \mathrm{H}), 7.11(\mathrm{~d}, \mathrm{~J}=8.7 \mathrm{~Hz}, 2 \mathrm{H}), 6.55(\mathrm{~d}, \mathrm{~J}=8.8 \mathrm{~Hz}, 2 \mathrm{H}), 4.88(\mathrm{dd}, \mathrm{J}=7.8$, $4.9 \mathrm{~Hz}, 1 \mathrm{H}), 3.25$ (t, J = 6.4 Hz, 2H), $2.10-1.99$ (m, 2H). GC/MS (m/z): 261).
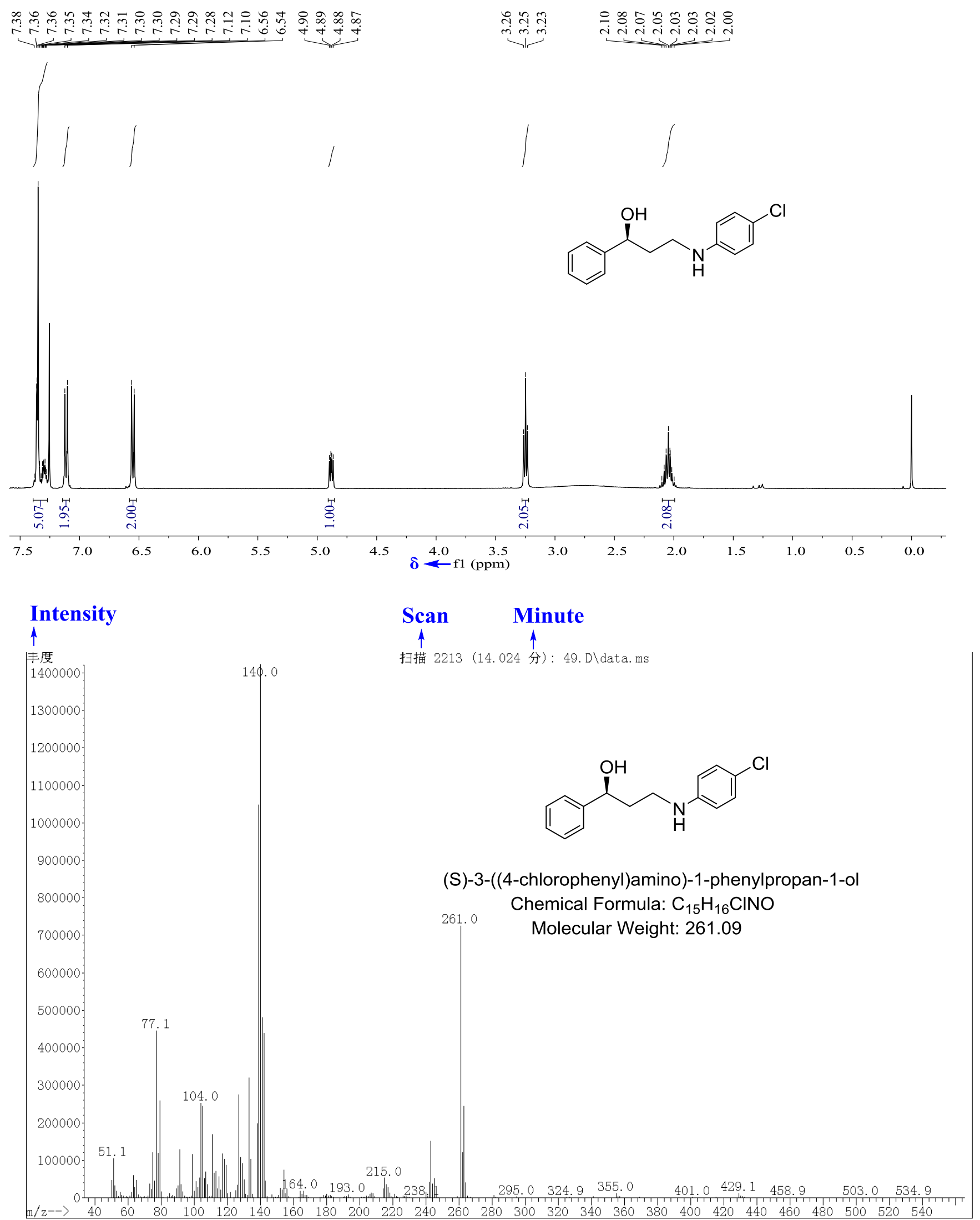
12j (entry 10 in Table 2): (S)-3-((3-chlorophenyl)amino)-1-phenylpropan-1-ol $\left({ }^{1} \mathrm{H}\right.$ NMR (400 MHz, Chloroform-d) $\delta 7.39-7.28(\mathrm{~m}, 5 \mathrm{H}), 7.05(\mathrm{t}, \mathrm{J}=8.0 \mathrm{~Hz}, 1 \mathrm{H}), 6.69-6.63(\mathrm{~m}, 1 \mathrm{H}), 6.56(\mathrm{t}, \mathrm{J}=2.1$ $\mathrm{Hz}, 1 \mathrm{H}), 6.48-6.44(\mathrm{~m}, 1 \mathrm{H}), 4.87(\mathrm{dd}, \mathrm{J}=7.8,4.8 \mathrm{~Hz}, 1 \mathrm{H}), 3.24(\mathrm{t}, \mathrm{J}=6.5 \mathrm{~Hz}, 2 \mathrm{H}), 2.10-1.98(\mathrm{~m}$, 2H). GC/MS (m/z): 261).

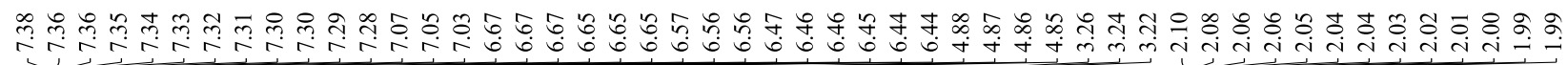
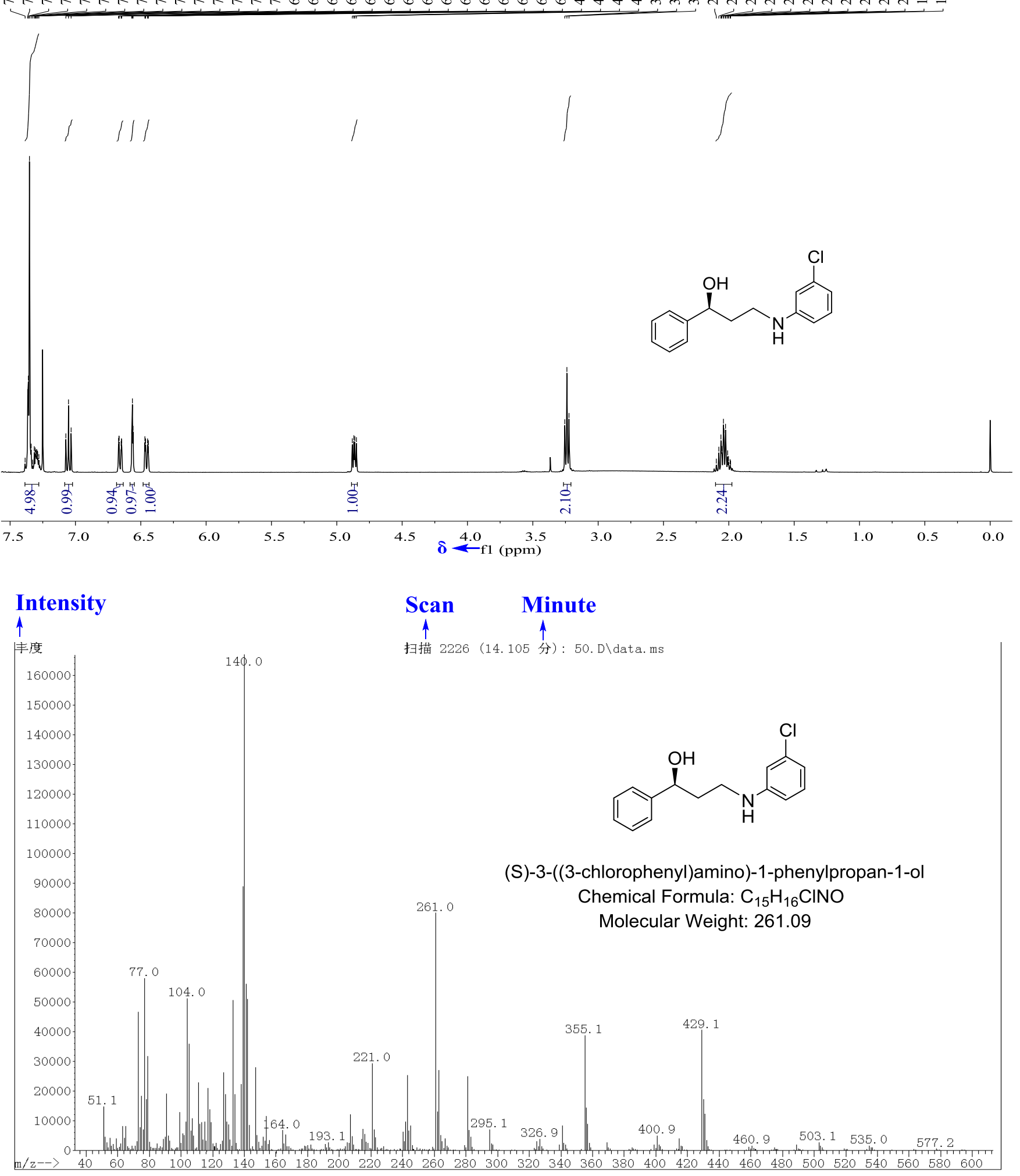
12k (entry 11 in Table 2): (S)-3-((4-bromophenyl)amino)-1-phenylpropan-1-ol ( ${ }^{1} \mathrm{H}$ NMR (400 MHz, Chloroform-d) $\delta 7.39-7.28(\mathrm{~m}, 5 \mathrm{H}), 7.23(\mathrm{~d}, \mathrm{~J}=1.7 \mathrm{~Hz}, 2 \mathrm{H}), 6.51(\mathrm{~d}, \mathrm{~J}=8.9 \mathrm{~Hz}, 2 \mathrm{H}), 4.87$ (dd, $\mathrm{J}=7.7,4.9 \mathrm{~Hz}, 1 \mathrm{H}), 3.24(\mathrm{t}, \mathrm{J}=6.4 \mathrm{~Hz}, 2 \mathrm{H}), 2.10-1.99(\mathrm{~m}, 2 \mathrm{H}) . \mathrm{GC} / \mathrm{MS}(\mathrm{m} / \mathrm{z}): 305)$.
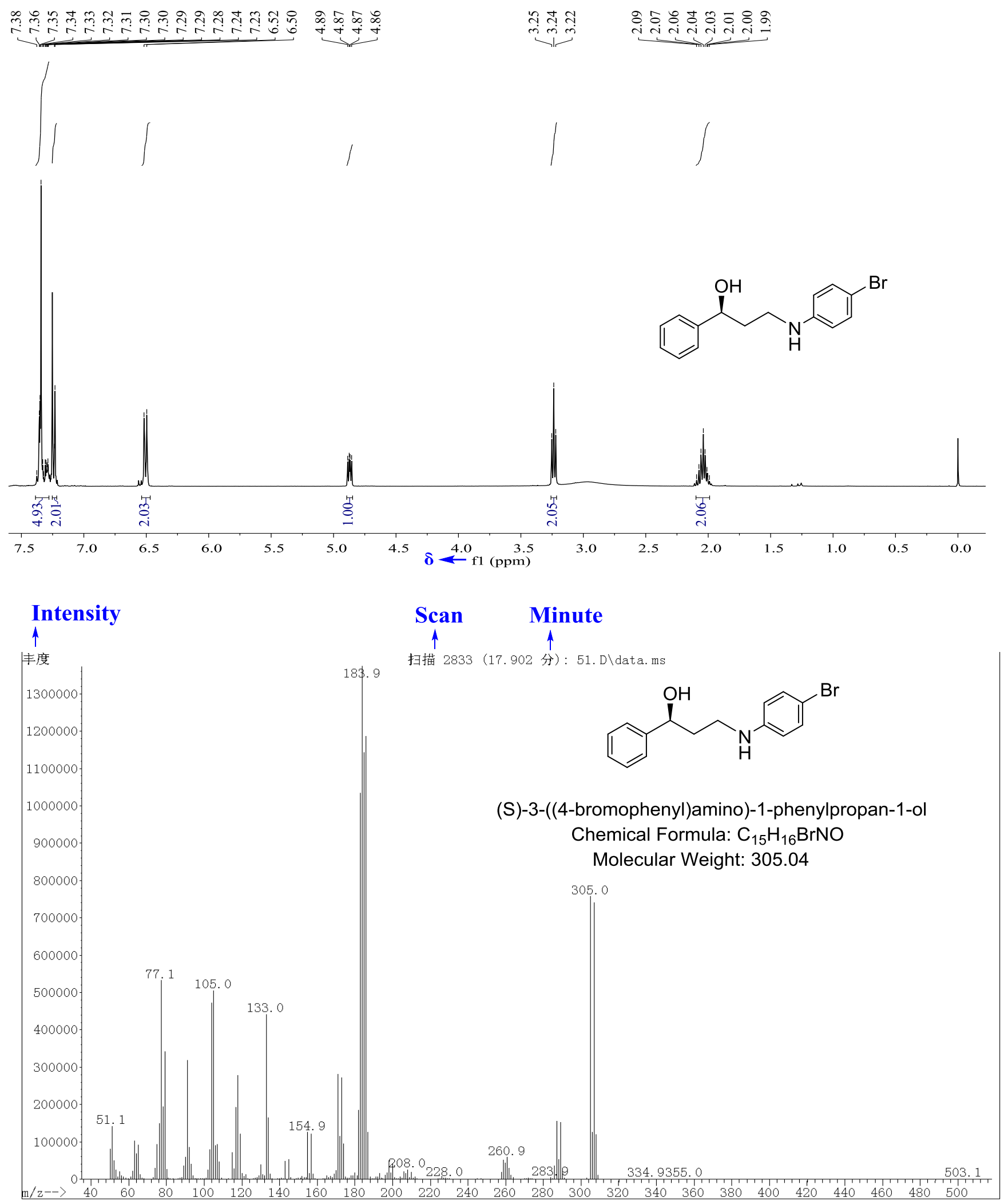
$12 \mathrm{l}$ (entry 12 in Table 2): (S)-1-phenyl-3-(p-tolylamino)propan-1-ol ( ${ }^{1} \mathrm{H}$ NMR (400 MHz, Chloroform-d) $\delta 7.29-7.20(\mathrm{~m}, 5 \mathrm{H}), 6.99(\mathrm{~d}, \mathrm{~J}=8.1 \mathrm{~Hz}, 2 \mathrm{H}), 6.82(\mathrm{~d}, \mathrm{~J}=8.3 \mathrm{~Hz}, 2 \mathrm{H}), 4.92-4.87$ (m, 1H), $3.29(\mathrm{t}, \mathrm{J}=6.3 \mathrm{~Hz}, 2 \mathrm{H}), 2.21(\mathrm{~s}, 3 \mathrm{H}), 2.04$ (q, J = 6.0 Hz, 2H). GC/MS (m/z): 241).
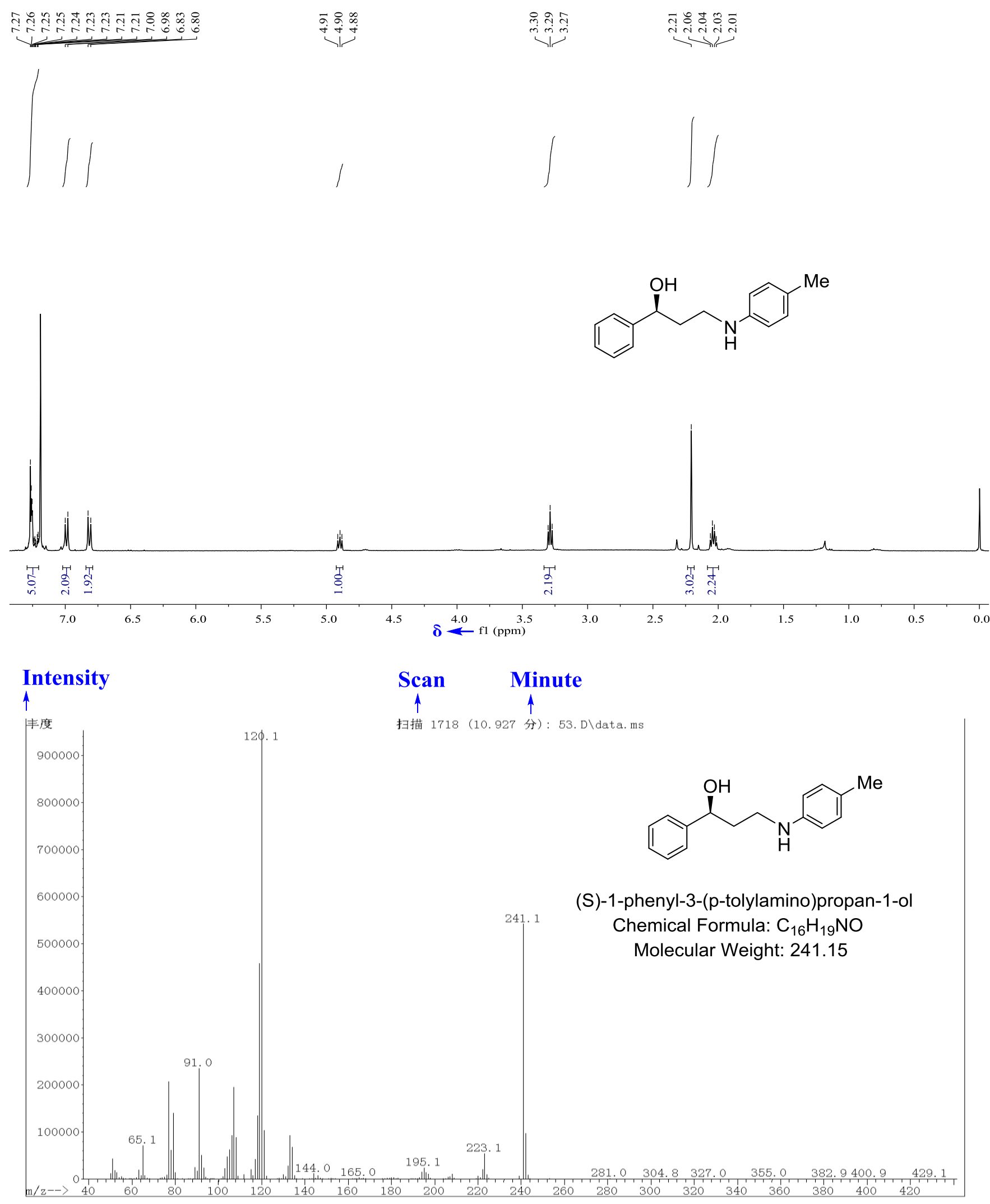
$12 m$ (entry 13 in Table 2): (S)-3-((4-methoxyphenyl)amino)-1-phenylpropan-1-ol ( ${ }^{1} \mathrm{H}$ NMR (400 $\mathrm{MHz}$, Chloroform-d) $\delta 7.32-7.17(\mathrm{~m}, 6 \mathrm{H}), 6.75-6.69(\mathrm{~m}, 2 \mathrm{H}), 6.59-6.53(\mathrm{~m}, 2 \mathrm{H}), 4.84(\mathrm{t}, \mathrm{J}=6.2 \mathrm{~Hz}$, 1H), $3.68(\mathrm{~s}, 3 \mathrm{H}), 3.18(\mathrm{t}, \mathrm{J}=6.2 \mathrm{~Hz}, 2 \mathrm{H}), 1.96(\mathrm{q}, \mathrm{J}=6.1 \mathrm{~Hz}, 2 \mathrm{H}) . \mathrm{GC} / \mathrm{MS}(\mathrm{m} / \mathrm{z}): 257)$.
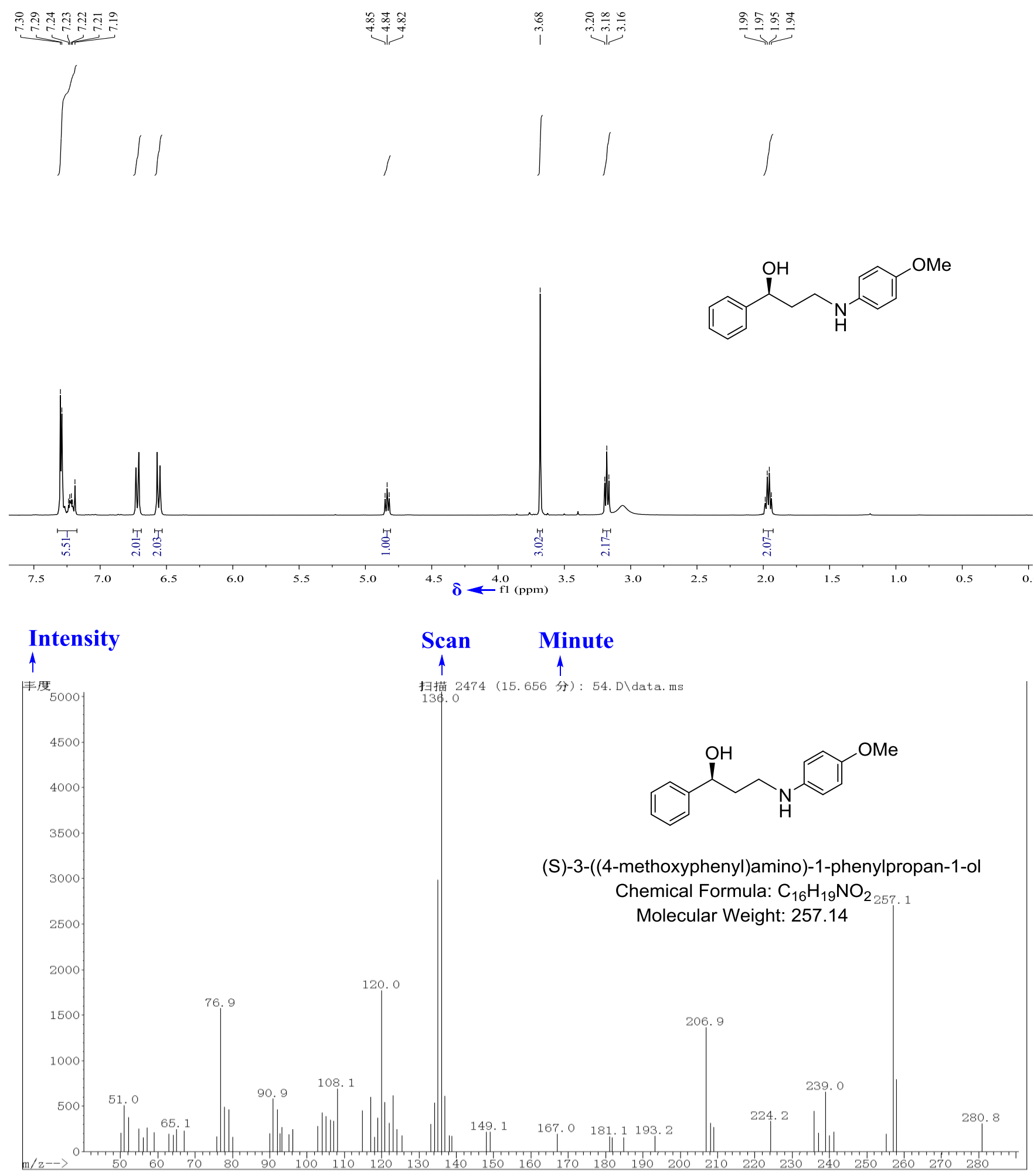

Figure S15. Characterizations of chiral products. 